\title{
Development of a Thermal-Mechanical Fatigue Testing Facility
}

\author{
by \\ Scott A. Yandt \\ B.Eng., Carleton University, 1998
}

\begin{abstract}
A thesis submitted to the Faculty of Graduate Studies and Research in partial fulfillment of the requirements for the degree of
\end{abstract}

\section{Master of Engineering}

Ottawa-Carleton Institute for Mechanical and Aerospace Engineering

Department of Mechanical and Aerospace Engineering

Carleton University

Ottawa, Ontario

August 2000

(C) Scott A. Yandt, 2000 
National Library of Canada

Acquisitions and Bibliographic Services

395 Wellington Street Ottawa ON K1A ON4 Canada
Bibliothèque nationale du Canada

Acquisitions et services bibliographiques

395, rue Wellington

Ottawa ON K1A ON4

Canada
The author has granted a nonexclusive licence allowing the National Library of Canada to reproduce, loan, distribute or sell copies of this thesis in microform, paper or electronic formats.

The author retains ownership of the copyright in this thesis. Neither the thesis nor substantial extracts from it may be printed or otherwise reproduced without the author's permission.
L'auteur a accordé une licence non exclusive permettant à la

Bibliothèque nationale du Canada de reproduire, prêter, distribuer ou vendre des copies de cette thèse sous la forme de microfiche/film, de reproduction sur papier ou sur format électronique.

L'auteur conserve la propriété du droit d'auteur qui protège cette thèse. Ni la thèse ni des extraits substantiels de celle-ci ne doivent être imprimés ou autrement reproduits sans son autorisation. 


\begin{abstract}
Components in the hot section of gas turbines undergo complex temperature-strain-time histories while in service. This leads to fatigue crack growth under conditions of varying temperature and cyclic strain, referred to as thermal-mechanical fatigue (TMF). A computerized TMF testing facility and testing procedures are described. The TMF testing facility is capable of conducting fully reversed strain-controlled, in-phase (IP) and out-ofphase (OP) TMF, and isothermal low-cycle fatigue (IT-LCF) in the $600^{\circ} \mathrm{C}$ to $1000^{\circ} \mathrm{C}$ temperature range. TMF and IT-LCF tests were performed on bare IN738LC nickel-base superalloy at strain ranges from 0.4 to 1 percent to commission the TMF testing facility. The tests were conducted in air, through a temperature range of $750^{\circ} \mathrm{C}$ to $950^{\circ} \mathrm{C}$ at a constant strain rate of $2 \times 10^{-5} \mathrm{sec}^{-1}$. The results of these tests demonstrate the successful application of the testing procedures and prove the testing system is capable of cycling specimens under various programmed thermal-mechanical loading histories. The fatigue lives were found to differ with strain-temperature phasing and strain range. The results of IP-TMF tests correspond well to the cyclic life observed during IT-LCF loading. Predominantly intergranular crack initiation and propagation were observed under all loading conditions.
\end{abstract}




\section{Acknowledgements}

I would like to express my gratitude to my advisor, Dr. Jonathan Beddoes, for providing me with the opportunity to work on this project. His continued guidance and constructive advice while working on this project is greatly appreciated. I would also like to thank Dr. Peter $\mathrm{Au}$, for his helpful supervision and the advice he provided during the course of this work.

The majority of the work for this thesis was performed at the Structures, Materials and Propulsion Laboratory of the Institute for Aerospace Research (IAR), National Research Council of Canada (NRC). I would also like to devote my sincere thanks to Tak Terada, Dave Morphy, Dave Chow, and the other staff of M-13 and M-14 who participated in the technical aspects of this project.

I would also like to thank my fellow graduate students Rick Kearsey and Henry Saari for their moral support and friendship.

Finally, I would like to thank my mother and father for their encouragement and support during the course of this work, and throughout my life. 


\section{Table of Contents}

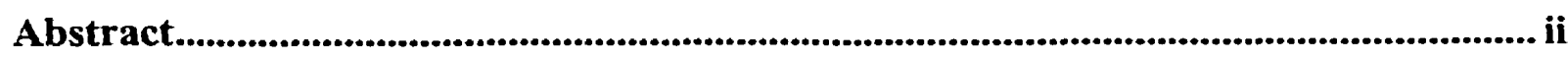

Table of Contents .............................................................................................................. iv

List of Tables ................................................................................................................... vii

List of Figures............................................................................................................................. viii

Appendices......................................................................................................................................... xiii

Nomenclature

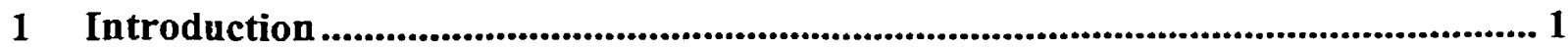

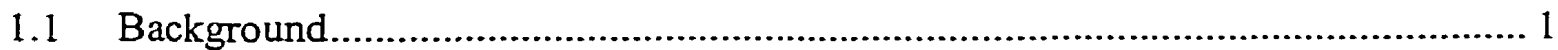

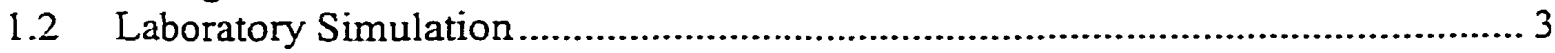

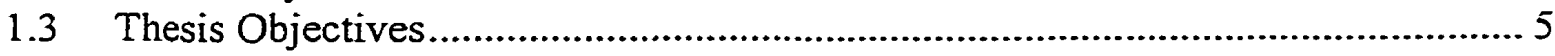

2 Review of LCF and TMF Behavior of Superalloys............................................... 10

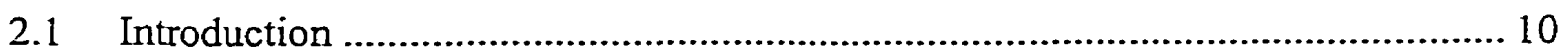

2.2 Microstructure of Nickel-Base Superalloys …................................................ 10

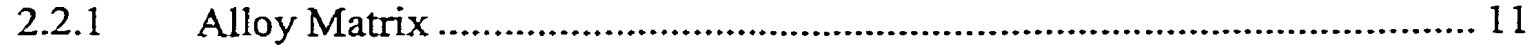

2.2.2 Precipitation Strengthening ................................................................ 12

2.2.3 Carbide Precipitation/Grain Boundary Strengthening............................... 13

2.3 Low Cycle Fatigue.................................................................................... 13

2.4 LCF at Elevated Temperatures .................................................................... 17

2.4.1 Effects of Frequency and Environment on LCF Life ............................. 18

2.5 Influence of TMF Temperature-Strain Relationships on TMF Life.................... 19

2.5.1 TMF Temperature -Strain Relationships ……….................................... 19

2.5.2 Comparison of TMF and IT-LCF Life .................................................. 20

2.6 Damage Mechanisms and Fatigue Life Under LCF and TMF Loading............. 21

2.6.1 Mechanisms of Fatigue Crack Initiation and Propagation in Superalloys 21

2.6.2 Creep-Fatigue Interaction ...................................................................... 23

2.6.3 Fatigue-Oxidation Interaction............................................................ 27

2.7 Microstructural Changes and Cyclic Response Under TMF and LCF Cycling.. 31

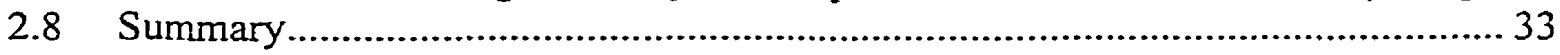

3 Review of Thermal-Mechanical Fatigue Testing Methods...................................... 46

3.1 Introduction to TMF Testing ........................................................................ 46

3.2 Stress-Controlled and Strain-Controlled TMF Testing ….................................. 48

3.2.1 Thermal Strain Compensation ................................................................... 51

3.3 Heating/Cooling Methods..................................................................................5

3.4 Specimen Design For TMF Testing …………............................................. 58

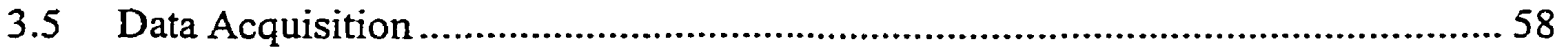




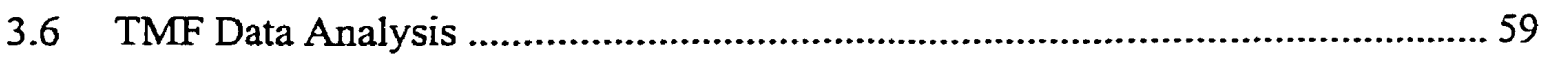

3.6.1 Stress-Strain Hysteresis Loop Components .............................................. 59

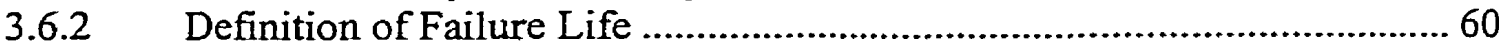

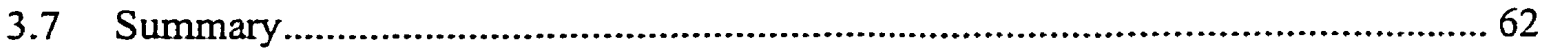

4 TMF Testing Facility and Experimental Approach ……....................................... 68

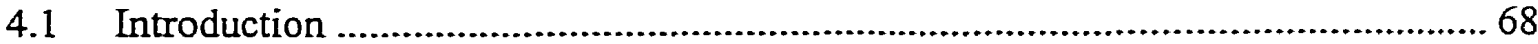

4.2 General Overview of the NRC-IAR TMF Testing Facility .................................69

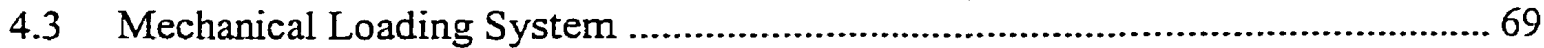

4.3.1 Modifications to MTS 407 Load Unit Controller..................................... 71

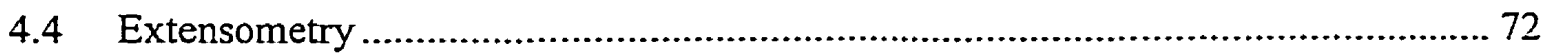

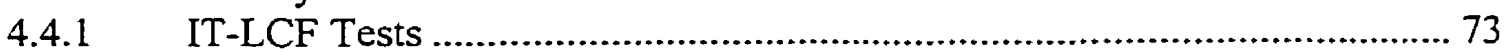

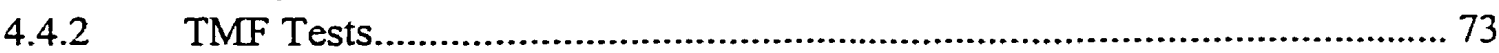

4.4.3 Thermal Strain Compensation ................................................................... 74

4.5 Specimen Heating/Cooling............................................................................ 75

4.5.1 Induction Heating ……………........................................................... 75

4.5.2 Heating Coils ..................................................................................... 76

4.5.3 Optimization of Gage Section Temperature Distribution............................ 78

4.5.4 Modifications to the RF Induction Heater ................................................. 80

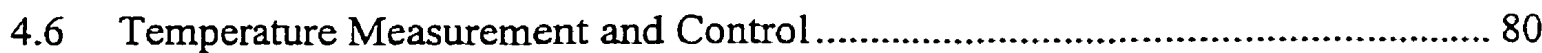

4.6.1 Temperature Control Loop ………………......................................... 81

4.6.2 Optical Pyrometry.................................................................................... 81

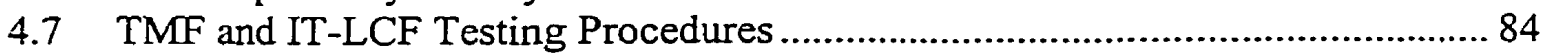

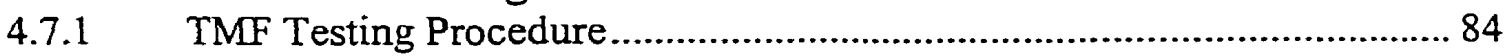

4.7.2 IT-LCF Testing Procedures .................................................................. 85

4.8 Data Acquisition System and TMF Testing Software ........................................ 85

4.8.1 LabVIEW IT-LCF/TMF Testing Software ………….............................. 86

4.8.2 Noise Considerations for Analog Signals............................................... 89

4.9 Data Analysis Procedures ............................................................................. 91

4.9.1 Hysteresis Loop Components ............................................................. 91

4.9.2 Determination of Failure .................................................................... 93

4.9.3 Presentation of Results ........................................................................... 93

5 IT-LCF and TMF Commissioning Tests............................................................. 108

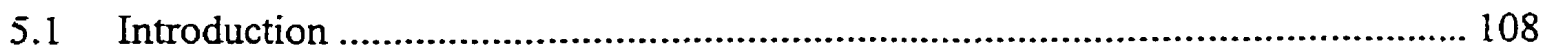

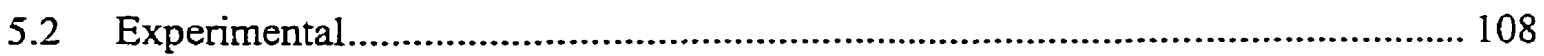

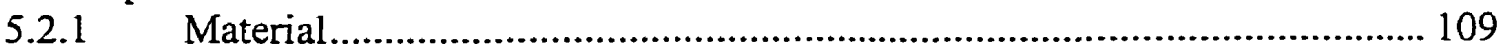

5.2.2 Test Specimens ............................................................................. 110

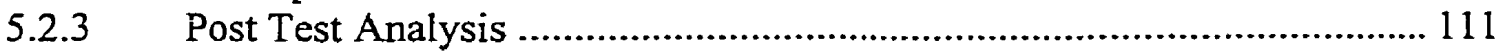

5.3 Results and Discussion ........................................................................... 111

5.3.1 Stress-Strain Behavior and Fatigue Life.............................................. 111

5.3.2 Microstructural Observations .......................................................... 114

6 Summary and Recommendations for Future Work ........................................... 133

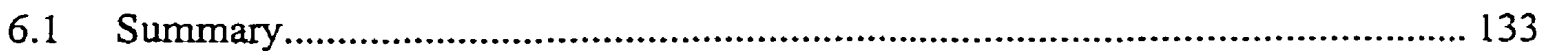

6.2 Recommendations for Future Work ……................................................. 134 
References ............................................................................................................. 136 


\section{List of Tables}

Table 4-1 - Bending strain readings taken before the commissioning test program using 12 strain gage specimen and $5 \mathrm{kN}$ axial load.......................................................... 94

Table 5-1 - Nominal composition of IN 738LC in wt\%...................................... 118

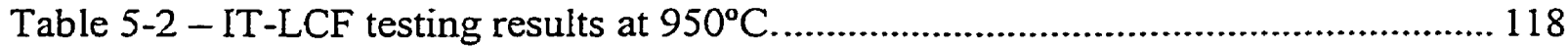

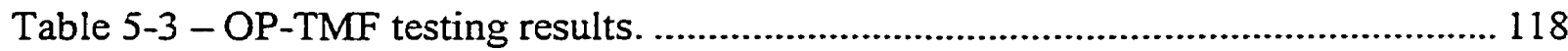

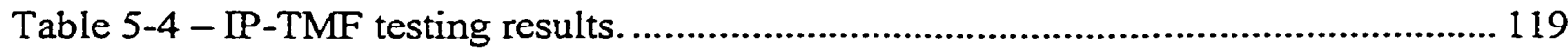




\section{List of Figures}

Figure 1-1 - Relationship between turbine inlet temperature and material introduction (140 MPa - 100 h capability); adapted from Reference 2.

Figure 1-2 - High pressure nozzle guide vane and turbine blade cooling arrangement ${ }^{3} \ldots 7$

Figure 1-3 - Leading (a) and trailing edge (b) cracking in a nozzle guide vane segment from an Allison T-56 turbo-propeller engine due to TMF.

Figure 1-4 - Strain-temperature plot for a blade leading edge during a startupacceleration-shutdown sequence; adapted from Reference 5.

Figure 1-5 - Isothermal and thermal-mechanical fatigue lives for various nickel, nickeliron, and cobalt-base superalloys ${ }^{9}$.

Figure 1-6- Historical sketch of test techniques to investigate transient thermal loading ${ }^{10}$.

Figure 2-1 - Effect of $\mathrm{Al}+\mathrm{Ti}$ content on creep rupture strength of nickel-base superalloys at $870^{\circ} \mathrm{C}^{15}$

Figure 2-2 - Strength (hardness) versus $\gamma^{\prime}$ precipitate diameter in a nickel-base superalloy ${ }^{15}$.

Figure 2-3 - Total strain range as sum of elastic and inelastic strain range components versus life piotted using logarithmic coordinates ${ }^{18}$

Figure 2-4 - Variation in number of cycles to failure with temperature for several nickelbase superalloys tested at a total constant strain range of $2.2 \%{ }^{23}$

Figure 2-5 - 0.2 percent offset flow stress as a function of temperature for several nickelbase superalloys ${ }^{23}$.

Figure 2-6 - Plastic strain range versus fatigue life for $\mathrm{A} 286$ in air and vacuum at $593^{\circ} \mathrm{C}$. Numbers adjacent to test points indicate frequency in cycles per minute $(\mathrm{cpm})^{22} \ldots \ldots \ldots . . .36$

Figure 2-7 - Strain versus time, and strain versus temperature relationships for $O P$, IP and CCWD-TMF cycles $^{33}$.

Figure 2-8 - Stress-mechanical strain response and damage mechanisms operative under in-phase and out-of-phase thermal-mechanical fatigue cycles ${ }^{42}$.

Figure 2-9 - (a) Accelerated oxidation of MC carbide (arrow) at surface of Mar-M200 at $925^{\circ} \mathrm{C}$. (b) Accelerated oxidation of grain boundary in Udimet 700 at $760^{\circ} \mathrm{C}^{15}$. 39

Figure 2-10 - Stress-mechanical strain hysteresis loops for an in-phase (left) and out-ofphase (right) TMF test ${ }^{56}$. 
Figure 2-11 - Influence of phase relation and maximum cycle temperature on the development of intergranular damage $e^{40}$

Figure 2-12 - Effect of oxygen activisity on transient and steady state oxidation of CMSX3 at $1200^{\circ} \mathrm{C}^{63}$

Figure 2-13 - Microstructure of $\mathrm{N}^{-}-738$ after $24 \mathrm{~h}$ oxidation at $975^{\circ} \mathrm{C}$ showing Ti-rich $\mathrm{Cr}_{2} \mathrm{O}_{3}$ scale, internal oxidation, and $\gamma^{\prime}$ depleted zone ${ }^{64}$.

Figure 2-14 - The effect of temperature on the fracture strains of chromium oxides formed on Nimonic $75^{68}$ 41

Figure 2-15 - Mechanisms of scallopøing: (a) mode 1; (b) mode $2^{71}$. 42

Figure 2-16 - Fatigue-oxidation cræack growth mechanism by embrittled oxide spike fracture. a) oxygen diffuses into the metal in front of the oxide spike tip; b) the embrittled metallic zone fractures; c) the fresh fractured metallic surfaces oxidizes and the oxygen diffuses into the metal again; process repeats as sketched in d), e) and f) ${ }^{33}$.......

Figure 2-17 - Fatigue-oxidation crack growth mechanism by oxide intrusion of Type I (A) and Type II (B) after Sehitoglua et al. $^{74}$. The oxide intrusion mechanism process occurs by the following steps: a) uni iform oxidation layer forms on the surface; b) when the oxide reaches some critical thiclkness, $h_{\mathfrak{t l}}$, the oxide ruptures and crack nucleation occurs; c) the fresh metallic surface ins exposed to the environment and oxidizes quickly; d) when the oxide reaches some critical thickness, $h_{\mathfrak{f} 2}$, the oxide again ruptures. The process and repeats as shown in $\mathrm{e}$ ) an $\mathrm{d} f$ )...... 44

Figure 2-18-Plasticity $(a, b, c)$ and oxide induced $(d, e, f)$ crack tip blunting ${ }^{72}$. 45

Figure 2-19 - Peak stress versus cycl e plot for $400^{\circ} \mathrm{C}$ to $600^{\circ} \mathrm{C}$ in-phase and out-of-phase TMF tests and bounding isothermal tests ${ }^{76}$

Figure 3-1 - Schematic view of a two -bar structure for TMF testing ${ }^{77}$ 63

Figure 3-2 - S-N results for the TMF= interlaboratory test program conducted under Cost 501 Round 2 using the same temperature and mechanical strain cycles ${ }^{81}$. Note: each code in the legend refers to the different pa:rticipant's project designation.

Figure 3-3 - Comparison between in-phase and out-of-phase TMF and bithermal fatigue cycles $^{88}$.

Figure 3-4 - Relationship between thermal, mechanical and total strain components for an out-of-phase TMF test.

Figure 3-5 - Relationship between thermal, mechanical and total strain components for in-phase TMF tests. 65

Figure 3-6 - Basic principles of direct induction heating ${ }^{98}$ 65 
Figure 3-7 - Specimen before and after cyclic barreling induced by $\mathrm{TMF}^{89}$ 66

Figure 3-8 - Tubular TMF specimen used by Remy ${ }^{10}$; others employ similar tubular specimen geometries for TMF testing. Notice the extended gage section used to facilitate obtaining a uniform axial temperature gradient. 66

Figure 3-9 - A schematic view of a TMF hysteresis loop defining the stress and strain parameters.

Figure 3-10 - Hysteresis loops formed during LCF testing illustrating stress amplitude (tensile) drop and cusp formation associated with the presence of a macrocrack. 67

Figure 4-1 - General layout of the IAR-NRC thermal-mechanical fatigue testing facility.

Figure 4-2 - Control block diagram for the TMF testing facility illustrating the temperature and total axial strain control loops. 95

Figure 4-3 - Strain gage alignment specimen for setting the concentric and angular alignment of the load train.

Figure 4-4 - Interlock chain connected to MTS 407 controller remote emergency stop connector.

Figure 4-5 - Schematic view of PC/MTS 407 controller interlock sense connection...... 97

Figure 4-6 - Close-up view of $25 \mathrm{~mm}$ high temperature axial extensometer and induction coil used for isothermal LCF testing.

Figure 4-7 - Close-up view of radiation pyrometer sensing head, $12 \mathrm{~mm}$ high temperature axial extensometer and induction coil for TMF testing. 98

Figure 4-8 - Stress induced while compensating for thermal strain during thermal cycling. 99

Figure 4-9 - Measured dynamic axial temperature distribution for $750^{\circ} \mathrm{C}$ to $950^{\circ} \mathrm{C}$ temperature range and temperature rate of $1^{\circ} \mathrm{C}$ per second.

Figure 4-10 - Thermocouple configuration for determining axial temperature gradients.

Figure 4-11 - Modifications to induction heater heat-on/off panel to provide an interlock connection to the MTS 407 controller and run/stop connection to the data acquisition PC. 100

Figure 4-12 - Schematic view of the closed-loop temperature control system. 101

Figure 4-13 - Plot illustrating the drift observed in the extensometer feedback signal when an IN $738 \mathrm{LC}$ specimen was heated to $950^{\circ} \mathrm{C}$ and held constant for 3 hours. 101 
Figure 4-14 - Thermocouple setup for calibrating the infrared pyrometer E-slope. 102

Figure 4-15 - E-slope calibration curve for the infrared pyrometer used in the present study, showing the difference between the average thermocouple temperature reading and the temperature indicated by the infrared pyrometer.

102

Figure 4-16 - Temperature command and feedback response of the infrared pyrometer temperature controller for a temperature range of $750^{\circ} \mathrm{C}$ to $950^{\circ} \mathrm{C}$ and a cycle period of 450 seconds. 103

Figure 4-17 - Example of a LabVIEW front panel (A) and block diagram (B) for calculating a triangular waveform. 104

Figure 4-18 - User display screen for LabVIEW TMF testing software during a test. .. 105

Figure 4-19 - Analog signal noise from the loadcell signal conditioner after averaging 80 readings (A) and the maximum peak-to-peak signal noise as a function of the number of samples averaged for the temperature, extensometer and loadcell feedback signals (B).

Figure 4-20 - Typical variation in elastic modulus, for the IN738LC material used in the present study, with temperature determined before starting a TMF test. 107

Figure 4-21 - Definition of 5\% tensile stress amplitude change based on the intersection point of a line tangent to the uniform cyclic response region, and a line tangent to rapid load drop region of the cyclic stress response. 107

Figure 5-1 - SEM micrograph illustrating bimodal $\gamma^{\prime}$ distribution in standard heat-treated IN738LC.

Figure 5-2 - (A) Longitudinal and (B) transverse section through specimen blank revealing the grain size of the investment cast IN738LC used in this investigation..... 120

Figure 5-3 - (A) SEM micrograph illustrating script MC carbides and grain boundary morphology. (B) A higher magnification view of a grain boundary showing discrete $\mathrm{M}_{23} \mathrm{C}_{6}$ carbides.

Figure 5-4 - Specimen design for IT-LCF testing.

Figure 5-5 - Specimen design for TMF testing.

Figure 5-6 - Experimental stress-strain hysteresis loops representative of in-phase TMF, out-of-phase TMF, and isothermal-LCF tests at $1 \%$ mechanical strain range. 123

Figure 5-7 - Half-life cyclic stress-strain relationship ( $\sigma_{\text {tensile }}$ versus $\Delta \varepsilon_{\text {mech }}$ ) for isothermal-LCF, out-of-phase TMF and in-phase TMF tests. Note: the points shown on this figure are based on the average stress amplitude for two or three tests. 124 
Figure 5-8 - Typical cyclic stress response for isothermal-LCF, in-phase TMF and outof-phase TMF tests at $1 \%$ mechanical strain range.

Figure 5-9 - Mechanical strain range versus life to 5\% tensile stress amplitude drop for isothermal-LCF, in-phase TMF and out-of-phase TMF tests 126

Figure 5-10 - Inelastic strain range versus life to 5\% tensile stress amplitude drop for inphase TMF, out-of-phase TMF, and isothermal-LCF tests.

Figure 5-11 - Inelastic strain range plotted as a function of mechanical strain range for isothermal LCF, in-phase TMF and out-of-phase TMF tests. Note: the points shown on this figure are based on the average inelastic strain range for two or three tests. 128

Figure 5-12 - Intergranular fatigue crack initiation sites observed in (A) isothermal-LCF (arrow), (B) out-of-phase TMF, and (C) in-phase TMF specimens. 129

Figure 5-13 - (A) An example of (1) transgranular, and (2) intergranular fatigue crack propagation. (B) A close-up view of an intergranular fatigue crack, and (C) mixed intergranular-transgranular fatigue crack.propagation. 130

Figure 5-14 - (A) Micrograph illustrating intergranular damage observed in in-phase TMF tests, and B) enlarged view of region shown on (A) illustrating decohesion at carbide-grain boundary interface and triple point wedge cracking. 131

Figure 5-15 - Changes to $\gamma^{\prime}$ precipitate morphology after A) isothermal-LCF, B) in-phase TMF, and C) out-of-phase TMF cycling. 


\section{Appendices}

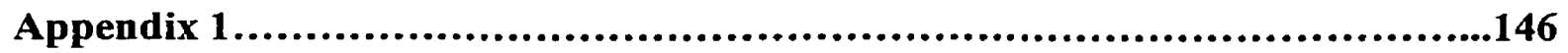

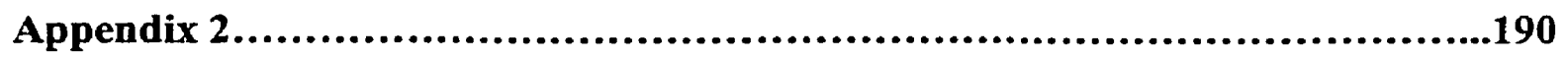

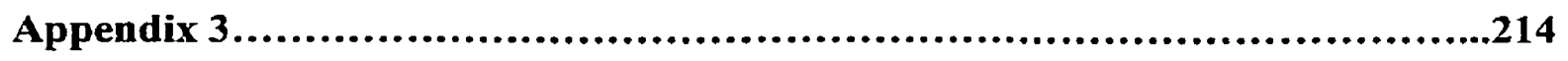




\section{Nomenclature}

A: Area

AI: Analog Input

AO: Analog Output

ASTM: American Society for Testing and Materials

C: Linear thermal expansion Constant

CC: Conventionally Cast

CCWD: Counter Clockwise Diamond

DAQ: Data Acquisition

DS: Directionally Solidified

E: Elastic Modulus

ECTE: Effective Coefficient of Thermal Expansion

FCC: Face Centered Cubic

FEM: Finite Element Method

h: Hours

HP: High Pressure

$\mathrm{Hz}$ : Hertz

IAR: Institute for Aerospace Research

ID: Internal Diameter

I/O: Input/Output

IP: In-Phase

IT-LCF: Isothermal Low-Cycle Fatigue

LCF: Low Cycle Fatigue

LVDT: Linear Variable Differential Transformer

$\mathrm{n}$ : Number of readings averaged

$\mathrm{N}$ : Number of cycles

$\mathrm{N}_{95}:$ Number of cycle to $5 \%$ tensile stress drop

$N_{f}:$ Number of cycles to fracture

$\mathrm{N}_{\mathrm{v}}$ : Electron vacancy number

NGV: Nozzle Guide Vane 
NI: National Instruments

NRC: National Research Council of Canada

OP: Out-of-Phase

$\Delta \mathrm{P}$ : Load amplitude

R: Strain ratio $\left(\varepsilon_{\min } / \varepsilon_{\max }\right)$

RF: Radio Frequency

S: Stress

sec: Seconds

SFE: Stacking Fault Energy

SRP: Strain Range Partitioning

SX: Single Crystal

t: Time

$\mathrm{T}$ : Temperature

TF: Thermal Fatigue

TIT: Turbine Inlet Temperature

TMF: Thermal-Mechanical Fatigue

TTL: Transistor-Transistor Logic

VB: Visual Basic

VI: Virtual Instrument

VDC: Volts Direct Current

$\alpha$ : Effective coefficient of thermal expansion

$\varepsilon:$ Strain

$\varepsilon_{\text {in: }}$ Inelastic strain

$\varepsilon_{\text {tot }}\left(\right.$ or $\left.\varepsilon_{\mathrm{t}}\right)$ : Total strain

$\varepsilon_{\mathrm{e}}$ : Elastic strain

$\varepsilon_{\text {mech }}:$ Mechanical strain

$\nu_{\mathrm{p}}:$ Plastic (inelastic) Poisson ratio

$v_{\mathrm{e}}$ : Plastic Poisson ratio

$\sigma:$ Stress 


\section{Introduction}

\subsection{Background}

During the past 60 years, there has been an unrelenting drive to increase the performance and improve the thermal efficiency of land-based, and aircraft gas turbine engines. One key aspect for realizing this goal has been to increase the turbine inlet temperature (TIT); however, the TIT is limited by the materials used for the high pressure (HP) turbine biades and nozzle guide vanes (NGV's). At present, the materials used in the turbine section are nickel or cobalt-base superalloys, owing to their outstanding strength at the temperature ranges at which gas turbine components operate ${ }^{1}$. As shown in Figure $1-1^{2}$, there has been a continuous improvement in the temperature capability of nickel-base superalloys; however, the most significant contribution to the increasing TIT's, has been the introduction of continuous air cooling of HP turbine blades and NGV's. Continuous air cooling has the effect of lowering the average section temperature, and has evolved to the point where these components can be operated in an environment where the gas temperature exceeds the materials melting point without compromising their integrity.

Cooling air is supplied to these components by directing a portion of the compressor air through series of intricate internal passages within the NGV's, and turbine airfoils, as illustrated in Figure $1-2^{3}$. As well as introducing stress concentration features, cooling passages contribute to substantial variations in temperature, which occur over small 
regions of the component cross section ${ }^{4}$. Therefore, in addition to centrifugal stresses, and gas bending loads, turbine blades and NGV's experience severe thermal loads, which vary considerably during the operating cycle of the engine. These rapidly changing external conditions result in transient temperatures and induced thermal stresses of sufficient severity to cause failure in extremely short periods ${ }^{4}$. The thermal and mechanical loads can both vary markedly during the operating cycle, and are cyclic in nature. Therefore, cyclic damage induced under transient thermal and mechanical loading has been termed Thermal-Mechanical Fatigue (TMF). Damage arising from TMF manifests itself as cracking at the leading and trailing edges of turbine blades and NGV's. Figure 1-3 illustrates leading and trailing edge cracks in a NGV segment due to TMF damage.

The actual temperature-strain history for an airfoil during an operating cycle is very complex. Figure $1-4^{5}$ shows the temperature strain history for the leading edge of a cooled airfoil resulting from one cycle of operation consisting of startup, acceleration, loading, unloading, and shutdown. In many cases, the critical areas of components subjected to thermally induced strains have been designed based on the results of uniaxial isothermal low cycle fatigue (IT-LCF) tests at the maximum expected service temperature. In the IT-LCF test, a uniaxial test specimen is used to simulate a small element of material in a critical location of a component subjected to cyclic temperature variations. The ASME Boiler and Pressure Vessel Code Case N-47 is an example of structural design rules that utilize strain-controlled LCF data to approximate the effect that transient thermal loads have on the lifetime of components ${ }^{6}$. The underlying 
assumption in the IT-LCF test conducted at the maximum service temperature is that it is representative of the 'worst case', and therefore will result in a conservative estimate of fatigue life. However, it is found that these tests are insufficient, because they do not discriminate enough for behavior evaluation, and are unrealistic for identifying the damage mechanisms during transient thermal and mechanical loading ${ }^{7}$. The synergy between fatigue damage and time-dependent phenomena, such as creep or oxidation, can be much stronger during thermal transient conditions than that observed under isothermal creep-fatigue loading ${ }^{8}$. Referring to Figure $1-5^{9}$, which lists the results for a number of turbine disk, vane and blade materials, it is clearly seen for a number of materials that ITLCF testing is inadequate for TMF life prediction. This has led the designers to use other means of mechanical testing to assess the damage induced by transient thermal stresses.

\subsection{Laboratory Simulation}

Various tests are used to simulate fatigue during transient thermal conditions. Consider Figure $1-6^{10}$, which shows the history of these test methods. The oldest of the laboratory simulation techniques is referred to as the thermal fatigue (TF) test. Spera ${ }^{\text {Il }}$ defines thermal fatigue as: "the gradual deterioration and eventual cracking of a material by alternate heating and cooling during which free thermal expansion is constrained." The constraint in this case can be either by internal or external sources. Glenny was the first to introduce thermal shock tests for investigating the behavior of wrought alloys used in turbine blades and vanes in gas turbines ${ }^{10}$. In these tests, wedge shaped disks are alternately immersed in heated and cooled beds of alumina particles, with air as the working fluid, which are referred to as fluidized beds. When the wedge geometry 
specimens are placed in the hot fluidized bed, the thin edges of the disk having lower thermal mass heat more quickly than the bulk. Therefore, internal constraint occurs since the thermal expansion of the edges is constrained by the cooler bulk of the disk. TF testing is particularly suited to component level testing. For example, Spera ${ }^{12}$ has applied TF testing to simulated turbine blades. The simulated blades were heated by a gas flame burner rig, and removed from the gas flame during the cooling portion of the test cycle. The major disadvantage of TF tests arises from the fact that the deformation occurs solely due to transient heating and cooling. Therefore, the cyclic stress and strain behavior must be computed by finite element methods (FEM), using transient thermal analysis to predict the temperature distribution, and sophisticated visco-plastic constitutive models to predict the stresses and strains arising from the predicted temperature distribution ${ }^{13}$.

The thermal-mechanical fatigue (TMF) test in many respects, is similar to the IT-LCF test; however, the test specimen is subjected to thermal cycling during mechanical loading. The constraint of free thermal expansion-contraction is imposed by an external system, such as a servo-hydraulic or electro-hydraulic fatigue machine. Like the IT-LCF test, the purpose of TMF tests is to simulate the behavior of a small element of material in a critical location where, cyclic deformation occurs by transient thermal cycling and mechanical loading. In TMF testing, total strain is the controlled variable, where total $\operatorname{strain}\left(\varepsilon_{\text {tot }}\right)$ is defined as:

$$
\varepsilon_{t o t}=\varepsilon_{m e c h}+\varepsilon_{t h}
$$


where $\varepsilon_{\text {mech }}$ is the applied mechanical strain, and $\varepsilon_{\text {th }}$ is the thermal strain (thermal expansion-contraction) of the tested material. Only the applied mechanical strain is 'seen' by the uniaxial specimen, and it simulates strains arising from mechanical loads and constrained thermal expansion. A faithful representation of the cyclic temperature and strain seen by a volume element is possible in the TMF test, since the temperature and constraint can be independently controlled. The primary advantage of the TMF test is that deformation (strain) is the controlled variable, and load (stress) can be monitored by a load cell in series with the uniaxial specimen. Therefore, the complex computational techniques used in TF tests are not necessary for TMF data analysis.

\subsection{Thesis Objectives}

To develop constitutive models to predict the behavior of single crystal superalloys, during transient thermal and mechanical loading, a joint project was established between Carleton University and the Institute for Aerospace Research of the National Research Council (IAR-NRC). This thesis was undertaken as part of this overall program with the objective of developing a TMF testing facility and experimental methods to support the work of this joint project. In Chapters 2 and 3, the background knowledge required to support the work in this thesis is presented through a comprehensive review of the literature. The review focuses on the different damage mechanisms and cyclic material response under TMF and IT-LCF cycling conditions, and the technical requirements for experimental facilities necessary to support constitutive model development. Chapter 4 discusses the experimental facility and experimental procedures developed as part of this thesis. Chapter 5 discusses the results of a test program to commission the TMF testing 
facility. The last Chapter summarizes the work completed, conclusions, and makes suggestions for further work. 


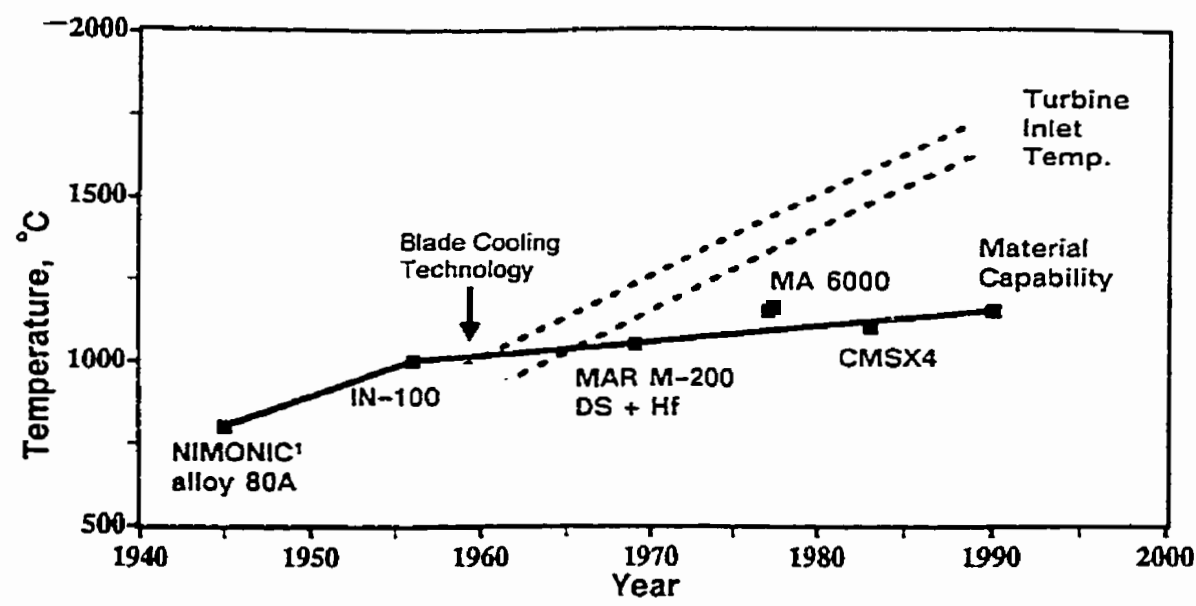

Figure 1-1 - Relationship between turbine inlet temperature and material introduction (140 MPa $-100 \mathrm{~h}$ capability); adapted from Reference 2.

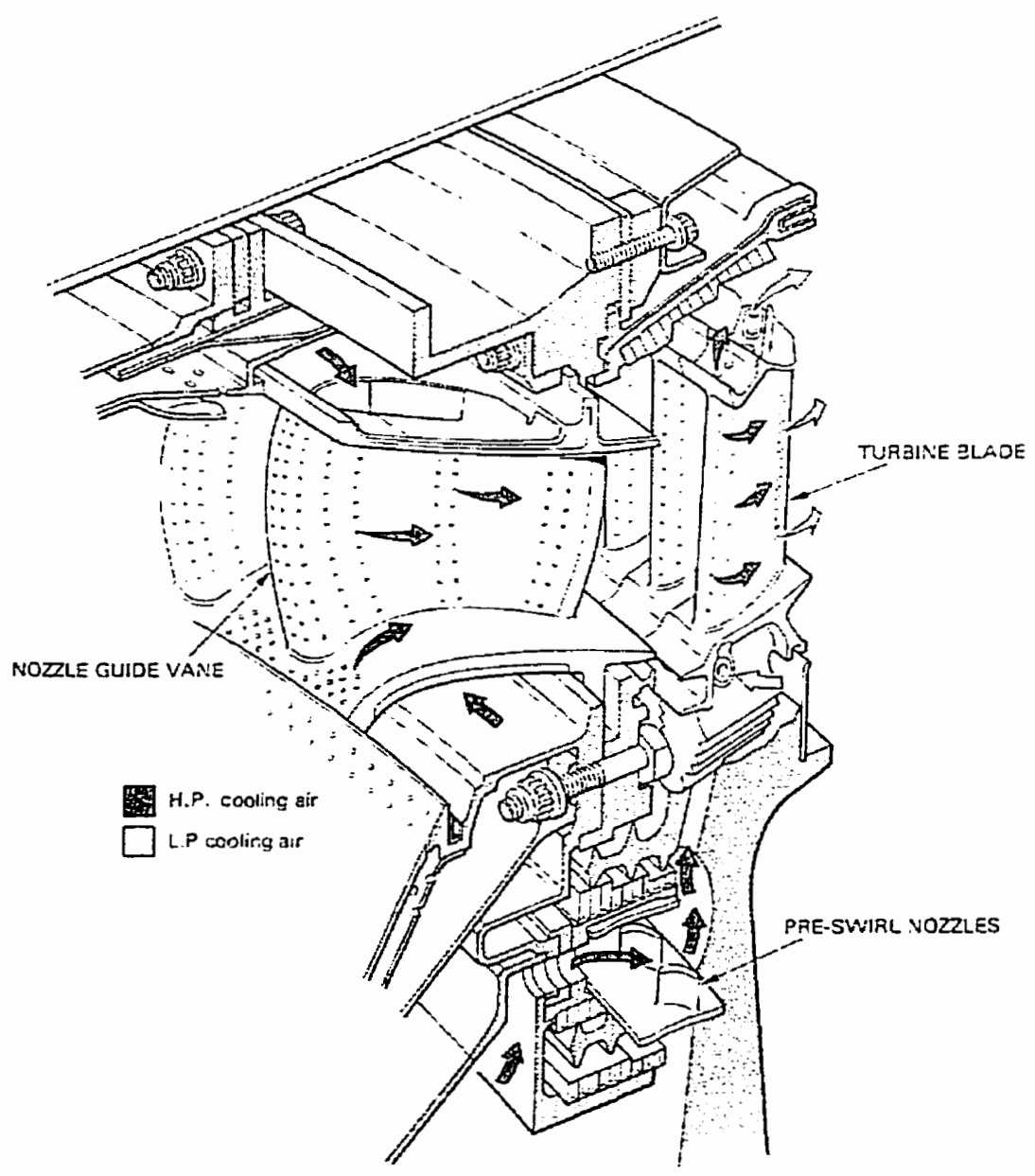

Figure 1-2 - High pressure nozzle guide vane and turbine blade cooling arrangement ${ }^{3}$. 

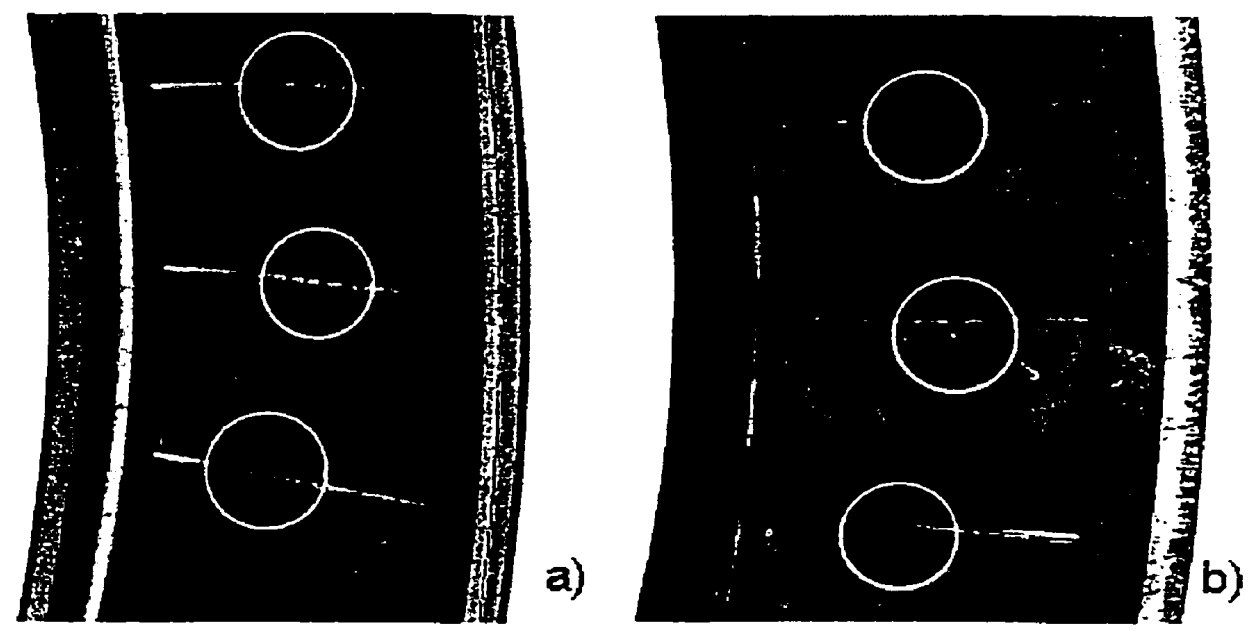

Figure 1-3 - Leading (a) and trailing edge (b) cracking in a nozzle guide vane segment from an Allison T-56 turbo-propeller engine due to TMF.

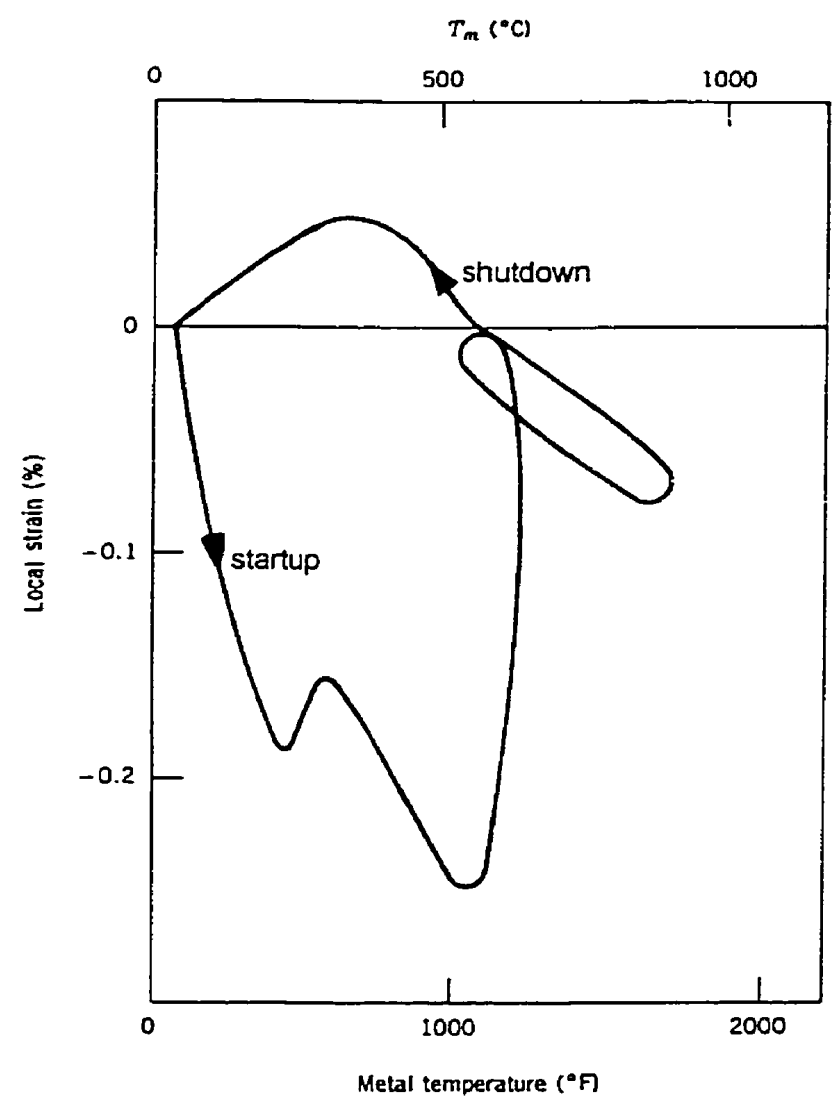

Figure 1-4 - Strain-temperature plot for a blade leading edge during a startupacceleration-shutdown sequence; adapted from Reference 5. 


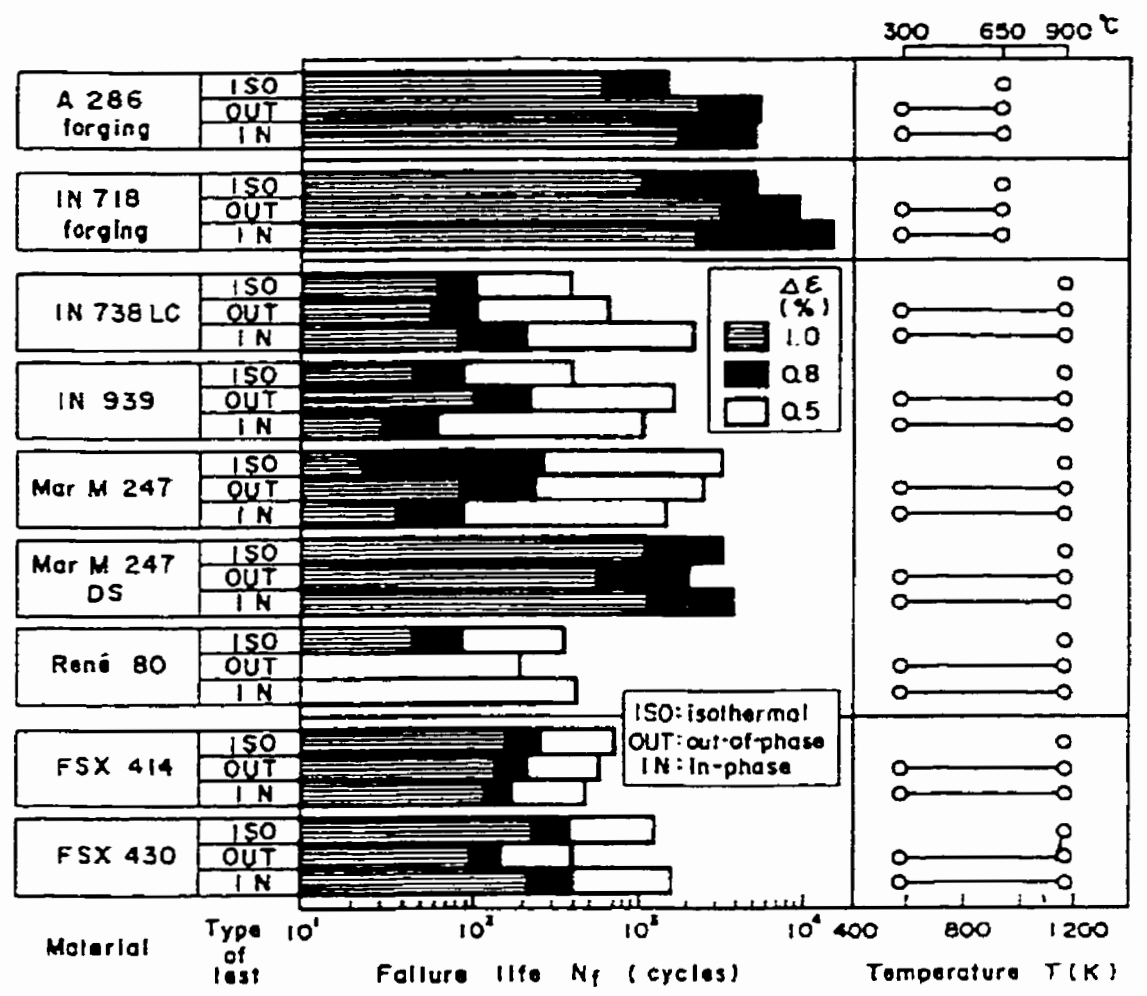

Figure 1-5 - Isothermal and thermal-mechanical fatigue lives for various nickel, nickeliron, and cobalt-base superalloys?

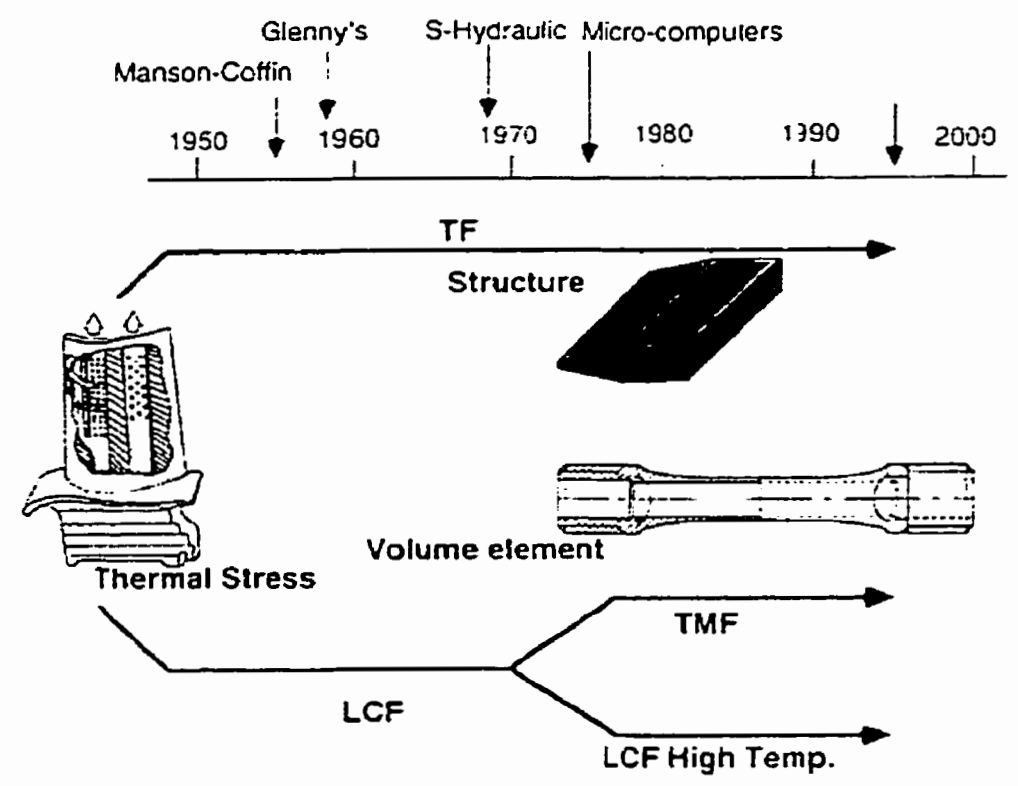

Figure 1-6- Historical sketch of test techniques to investigate transient thermal loading ${ }^{10}$. 


\section{Review of LCF and TMF Behavior of Superalloys}

\subsection{Introduction}

The following review focuses on the behavior of superalloys under TMF and IT-LCF cycling conditions. The first section briefly discusses the microstructure of nickel-base superalloys and its dependence on alloy composition. This is followed by a review of low temperature and elevated temperature LCF behavior of superalloys including, the effects of environment, and testing frequency. The behavior of superalloys under TMF and ITLCF cycling is discussed, including the role of TMF temperature-strain relationships on fatigue life. This is followed by a review of the observed modes of fatigue failure, and damage mechanisms operative under TMF and IT-LCF cycling conditions including, creep-fatigue, and fatigue-oxidation interaction. The last part of the review discusses the behavior of superalloys under TMF cycling conditions including, changes to microstructure and attendant changes to cyclic response.

\subsection{Microstructure of Nickel-Base Superalloys}

$\mathrm{Ni}$-base superalloys are a class of materials used in elevated temperature applications requiring outstanding high temperature strength, creep resistance, and resistance to oxidation and hot-corrosion attack. The resulting mechanical properties and microstructure of Ni-base superalloys are highly dependent on alloy chemistry, heat- 
treatment, and material processing. Chemically, these alloys can be extremely complex, containing controlled amounts of as many as $15-20^{1}$ elements. In general, the effects of alloying additions can be summarized into three major classifications based on function or how the elements partition into the various phases present in the alloy. The function of alloying additions to $\mathrm{Ni}$-base superalloys can be classified as follows:

\subsubsection{Alloy Matrix}

The alloy matrix of Ni-base superalloys is a face-centered-cubic (fcc) austenitic structure referred to as gamma $(\gamma)$. Ni-base superalloys owe part of their high temperature strength to the unique properties of the fcc matrix, which includes (1) high modulus, (2) low diffusivity for alloying additions, and (3) a high solubility for alloying elements ${ }^{14}$. Nickel-base superalloys are mainly comprised of nickel (30 to 75 wt.\%), along with appreciable amounts of chromium (up to $30 \%$ wt. $\%)^{15}$. Other elements (cobalt, molybdenum, tantalum, tungsten, and vanadium) are added to the $\gamma$ matrix for solid solution strengthening brought about by atomic size differences. The addition of solid solution strengthening elements has a second benefit, which is dependent on the elements placement on the periodic table, whereby an increasing the electron hole number, $N_{v}$, reduces stacking fault energy (SFE), making cross slip more difficult ${ }^{14}$. Aluminum and chromium additions to the $\gamma$ matrix improve oxidation and hot corrosion resistance, by forming adherent $\mathrm{Al}_{2} \mathrm{O}_{3}$ and $\mathrm{Cr}_{2} \mathrm{O}_{3}$ oxide scales when the alloy is exposed to elevated temperatures. 


\subsubsection{Precipitation Strengthening}

The addition of titanium and aluminum to the $\gamma$ matrix results in the precipitation of the ordered fcc intermetallic phase, denoted as gamma prime $\left(\gamma^{\prime}\right)$. The basic composition of $\gamma^{\prime}$ is $\mathrm{Ni}_{3}(\mathrm{Ti}, \mathrm{Al})$, with nickel occasionally being substituted by cobalt. Precipitate morphology is governed the mismatch between the $\gamma$-matrix and $\gamma^{\prime}$ precipitates. Small $\gamma / \gamma^{\prime}$ mismatches $(<0.5 \%)$ result in spherical $\gamma^{\prime}$, where as higher misfits $(0.5$ to $1.0 \%)$ result in the formation of cuboidal $\gamma^{16}$.

Although appreciable strengthening in superalloys is due to solid solution strengthening, almost all high temperature strength is due to the $\gamma^{\prime}$ precipitate. Consequently, the high temperature strength of Ni-base superalloys is strongly dependent on the volume fraction $\left(V_{f}\right)$, and the size (morphology) of the $\gamma^{\prime}$ precipitate. The $V_{f}$ of $\gamma^{\prime}$ is controlled by the amounts of aluminum and titanium added to the alloy (see Figure 2-1 ${ }^{15}$ ). The size (morphology) of the $\gamma^{\prime}$ precipitate is important when considering dislocation-precipitate interactions. When a mobile dislocation encounters a $\gamma^{\prime}$ precipitate, the dislocation either shears the precipitate ${ }^{14,15,16}$, or it bypasses the precipitate by Orowan looping ${ }^{14}$. When the precipitate size is small, shearing is the dominant strengthening mechanism. As the precipitate size increases, looping or other precipitate bypass mechanisms dominate, resulting in a loss in strength. Referring to Figure $2-2^{15}$, the optimum hardness (strength) of a Ni-base superalloy is controlled through careful control of the size of the $\gamma^{\prime}$ precipitate. Peak strength is usually attained at the transition point between precipitate cutting and looping mechanisms. 


\subsubsection{Carbide Precipitation/Grain Boundary Strengthening}

There are several types of carbides common to $\mathrm{Ni}$-base superalloys, such as $\mathrm{MC}, \mathrm{M}_{23} \mathrm{C}_{6}$ and $\mathrm{M}_{6} \mathrm{C}$, where $\mathrm{M}$ is $\mathrm{Cr}, \mathrm{Hf}, \mathrm{Ta}, \mathrm{Nb}, \mathrm{Ti}, \mathrm{W}, \mathrm{Mo}$ or a substitution of these elements. During solidification of the melt, heterogeneously scattered MC carbides form at the grain boundaries, and at intergranular sites ${ }^{16}$. At elevated temperatures, MC carbides are usually unstable, and will degrade to $\mathrm{M}_{23} \mathrm{C}_{6}$ at moderate temperatures. $\mathrm{MC}$ carbides may also degrade to $\mathrm{M}_{6} \mathrm{C}$ carbides provided that the alloy has sufficiently high $\mathrm{Mo}$ and $\mathrm{W}$ content $^{15,16}$. Controlled intergranular precipitation of carbides can impart higher strength in Ni-base superalloys. Discrete intergranular precipitation of $\mathrm{M}_{23} \mathrm{C}_{6}$ improves creep rupture strength by inhibiting grain boundary sliding. On the other hand, a continuous intergranular film of $\mathrm{M}_{23} \mathrm{C}_{6}$ reduces impact strength, and lowers creep rupture life ${ }^{15}$.

The additions of small amounts of $\mathrm{B}$ and $\mathrm{Zn}$ to $\mathrm{Ni}$-base superalloys has the beneficial effect of reducing the onset of grain boundary tearing under creep loading ${ }^{14}$. Two mechanisms have been proposed for the grain boundary strengthening effect of $\mathrm{B}$ and $\mathrm{Zn}$. First, the presence of $\mathrm{B}$ and $\mathrm{Zn}$ tends to tie-up tramp elements such as $\mathrm{S}$, and $\mathrm{Pb}^{15}$. Second, owing to the small size of the $\mathrm{B}$ and $\mathrm{Zn}$ atoms, it is thought that these elements fill discontinuities at the grain boundaries thus, lowering the grain boundary energy ${ }^{16}$.

\subsection{Low Cycle Fatigue}

In engineering structures localized regions of plastic (inelastic) deformation can occur at stress concentration features (notches) such as, bolt or rivet holes, or abrupt changes in section. Regardless of the loading condition in the structure (stress or strain), the loading 
condition at notches is predominantly a strain-controlled phenomenon. As mentioned previously, strain-controlled deformation may also arise when a structure is subjected to variations in temperature. Low-cycle fatigue at elevated temperatures will be considered in a later section.

During static loading, stress concentration features are generally not considered to be detrimental to the integrity of the structure. On the other hand, during cyclic loading conditions notches can initiate fatigue cracks, which progressively grow until the structure catastrophically fails. Therefore, the necessity to predict the fatigue behavior of materials used in engineering structures is of considerable importance. In general, fatigue damage under strain-controlled conditions, is treated by considering the cumulative effect of both the elastic and inelastic deformation components separately.

The elastic strain-life term is usually presented as the relationship between the true stress amplitude $\left(\sigma_{a}\right)$ and number of reversals to failure $\left(2 N_{f}\right)^{17}$. A linear relationship between the true stress amplitude and number of reversals to failure is realized when plotted on a $\log -\log$ scale. The elastic strain-life relationship is illustrated schematically on Figure $2-3^{18}$, which can be represented by the following equation

$$
\frac{\Delta \sigma}{2}=\sigma_{f}^{\prime}\left(2 N_{f}\right)^{b}
$$

where $\Delta \sigma / 2$ is the true stress amplitude, $2 \mathrm{~N}_{\mathrm{f}}$ is the reversals to failure ( 1 reversal $=1 / 2$ cycle), $\sigma_{f}^{\prime}$ is the fatigue strength coefficient, and $b$ is the fatigue strength exponent. The 
fatigue strength coefficient, $\sigma_{f}^{\circ}$, is approximately equal to the true fracture stress $\sigma_{t}$, and $\mathrm{b}$ is usually found to vary between -0.05 and $-0.12^{18}$. The true stress amplitude may be expressed in terms of strain amplitude, as follows:

$$
\frac{\Delta \varepsilon_{e}}{2}=\frac{\Delta \sigma}{2 E}
$$

where $E$ is the elastic modulus. Therefore, the elastic strain-life component can be expressed as

$$
\frac{\Delta \varepsilon_{e}}{2}=\frac{\sigma_{f}^{\prime}}{2 E}\left(2 N_{f}\right)^{b}
$$

The inelastic strain-life relationship, illustrated in Figure $2-3^{\mathrm{i}}$, is after Manson and Coffin ${ }^{19}$, who found that the inelastic strain-life component could be linearized as a power law function when plotting inelastic strain amplitude $\left(\Delta \varepsilon_{\text {in }} / 2\right)$ versus reversals to failure $\left(2 \mathrm{~N}_{f}\right)$ on a log-log scale. The Manson-Coffin equation is as follows:

$$
\frac{\Delta \varepsilon_{\text {in }}}{2}=\varepsilon_{f}\left(2 N_{f}\right)^{c}
$$

where $\Delta \varepsilon_{\text {in }} / 2$ is the inelastic strain amplitude, $2 N_{f}$ is the reversals to failure, $\varepsilon_{f}^{\prime}$ is the fatigue ductility coefficient, and $\mathrm{c}$ is the fatigue ductility exponent. The fatigue ductility coefficient, $\varepsilon_{f}^{\cdot}$, and fatigue ductility exponent, $\mathrm{c}$, are material dependant constants. The 
fatigue ductility coefficient, $\varepsilon_{f}^{\cdot}$, is approximately equal to the true fracture ductility evaluated from a monotonic tensile test, $\varepsilon_{\mathfrak{f}}$, and for many materials the fatigue ductility exponent, $c$, is found to range between -0.5 and $-0.7^{18}$.

Combining the elastic and inelastic terms gives the total strain-life $\left(\Delta \varepsilon_{t} / 2\right.$ versus $\left.2 \mathrm{~N}_{\mathrm{f}}\right)$ relationship illustrated in Figure 2-3 $3^{18}$, i.e.,

$$
\frac{\Delta \varepsilon_{t}}{2}=\frac{\sigma_{f}^{\prime}}{2 E}\left(2 N_{f}\right)^{b}+\frac{\dot{\varepsilon_{f}^{\prime}}}{2}\left(2 N_{f}\right)^{c}
$$

which, is the basis of the strain-life method. Further explanation of the coefficients in Equation 2-5, is found in the paper by Raske and Morrow ${ }^{20}$.

Referring to Figure $2-3^{18}$, two important observations can be made: (1) at high strain amplitudes the inelastic strain is the dominant term, and (2) there is a distinct crossover of the inelastic and elastic strain-life curves. The crossover of the inelastic and elastic strainlife curves is referred to as the transition point. For many materials at room temperature, the transition point corresponds to $10^{4}$ cycles $^{21}$. For fatigue lives less than the transition point, inelastic strain-life is the dominant strain-life component. Thus, low-cycle fatigue is generally considered to correspond to a fatigue life that is less than $10^{4}$ cycles, and the primary parameter, which governs low-cycle fatigue life, is considered to be inelastic strain. 


\subsection{LCF at Elevated Temperatures}

Superalloys are a class of materials, which are used at high temperatures and in aggressive environments. Primarily, one should address the predictive capability of the low-cycle fatigue strain-life relations at elevated temperatures. At elevated temperatures the first observed change to the strain-life plot is a decrease in transition life. For cast nickel-base superalloys, which combine high strength and low ductility, the transition life has been found to be substantially lower than $10^{4}$ cycles at high temperatures ${ }^{22}$. Furthermore, superalloys in general, show a fatigue life reduction when tested in air at elevated temperatures (see Figure $2-4^{23}$ ), even though the short-term tensile properties, e.g. yield strength and ductility, change little at moderate temperatures (less than $700^{\circ} \mathrm{C}$ ) (see Figure 2-5 ${ }^{23}$ ). Therefore, basing life predictions solely on the strain-life coefficients evaluated from high temperature tensile tests will over predict fatigue life ${ }^{24}$.

The fatigue life at elevated temperature is lower than predicted by tensile properties, due to various combinations of temperature, strain amplitude, and strain rate influencing the cyclic stress-strain response, combined with interaction of various time and temperature dependent mechanisms. The tensile properties are not affected by time-dependent damage to the same extent as observed in a low-cycle fatigue test, and therefore are inappropriate for estimation of fatigue life $^{19}$. The overall influence of time-dependant processes depends on factors such as temperature, frequency, hold times, mean stress, and environment ${ }^{25}$. The following subsection discusses the influence of frequency and environment on LCF life at elevated temperatures. 


\subsubsection{Effects of Frequency and Environment on LCF Life}

As temperature is increased, fatigue life is not only dependent on inelastic strain range, or number of cycles, but also on time per cycle (frequency). Numerous LCF studies have investigated the frequency sensitivity of superalloys at elevated temperatures ${ }^{22,26,27,28}$. In general, LCF life is found to be strongly sensitive to frequency, with fatigue life decreasing as testing frequency is reduced (see Figure 2-622. Also, as the testing frequency is decreased, the fracture mode is observed to change from predominantly transgranular to intergranular fatigue crack initiation and propagation. When the same LCF experiments are conducted in high vacuum, which would exclude the influence of environment, the fatigue life is found to be insensitive to frequency ${ }^{22,29,30,31}$ (see Figure $\left.2-6^{22}\right)$. These results suggest that the observed frequency sensitivity of LCF life is due to fatigue and environmental interaction.

This conclusion has been further substantiated by a number of investigators that exposed LCF specimens to an oxidizing environment prior to fatigue testing. Nazmy ${ }^{32}$ has demonstrated that $\mathrm{IN} 738 \mathrm{LCF}$ specimens pre-oxidized at $1000^{\circ} \mathrm{C}$ for $240 \mathrm{~h}$, followed by fatigue testing at $850^{\circ} \mathrm{C}$, substantially reduced the fatigue life. The fatigue crack initiation was found to be intergranular for both pre-oxidized and for specimens without prior exposure, but fatigue crack propagation was transgranular for specimens without priorexposure, and intergranular for pre-oxidized specimens. The difference in fatigue fracture mode observed in this study was associated with oxide embrittlement of the grain boundaries, since extensive grain boundary oxidation was observed in the pre-exposed specimens. In a similar study by Antolovich ${ }^{28}$, prior-exposure of Rene 80 LCF specimens 
to $982^{\circ} \mathrm{C}$ with $97 \mathrm{MPa}$ applied stress for $100 \mathrm{~h}$, substantially reduced the fatigue life over specimens without prior exposure. However, when the damage surface layer was removed by machining the specimens prior to LCF testing, the fatigue life was partially restored. Therefore, the effects of environment have a profound effect on both fracture mode, and LCF life.

\subsection{Influence of TMF Temperature-Strain Relationships on TMF Life}

\subsubsection{TMF Temperature-Strain Relationships}

Figure $2-7^{33}$ illustrates the IP (maximum strain coincides with maximum temperature), OP (maximum strain coincides with minimum temperature) and counter-clockwisediamond (CCWD, median strain coincides with the maximum and minimum temperatures) temperature-strain relationships typically used in TMF studies. Most investigators have based their studies on the in-phase (IP) and out-of-phase (OP) proportional temperature-strain relationships, and compared the fatigue life results obtained from these tests to IT-LCF lives at the maximum and minimum testing temperatures of the TMF cycle. Investigators have focussed on the IP and OP-TMF cycles since they represent the most arduous loading conditions, where tension occurs at high and low temperatures for each respective loading condition. The temperature-strain response of real components can be considered to consist of portions that are in-phase or out-of-phase (see Figure 1-4); however, purely IP and OP TMF cycles do not frequently occur in service. On the other hand, IP and OP TMF cycles are found to be useful for testing models and for evaluating new, advanced materials ${ }^{34}$. Since most investigations 
have focused on the IP and OP proportional loading, the remainder of this Chapter will focus on these two TMF loading conditions.

\subsubsection{Comparison of TMF and IT-LCF Life}

As previously mentioned, life prediction in thermal-mechanical fatigue problems, has been based on the results of IT-LCF tests at the maximum expected service temperature. It is anticipated that these tests are representative of the 'worst case', and will result in conservative estimates for fatigue life under transient thermal and loading conditions. On the contrary, many investigators ${ }^{7,35,36,37,38}$ have found that IT-LCF tests at the maximum TMF test temperature do not result in conservative life predictions for IP and OP-TMF

tests at the same mechanical strain amplitudes. Other investigators ${ }^{39,40,41}$ have found that IT-LCF tests provide an adequate estimate for either IP or OP-TMF life, but not for both TMF loading conditions.

A number of factors influence TMF life including temperature range, strain amplitude, and strain rate (testing frequency). The various combinations of these factors influence oxidation behavior, stress-strain response, and microstructural changes observed in the material. Typical damage mechanisms that may be operative in IP and OP TMF loading conditions are illustrated in Figure $2-8^{42}$. The interaction of these various mechanisms is complex, and exerts a strong influence on the observed fatigue fracture mode ${ }^{42}$. The following sub-sections will attempt to summarize from the literature the mechanisms of fatigue crack initiation and propagation that have been observed under LCF and TMF loading conditions. 


\subsection{Damage Mechanisms and Fatigue Life Under LCF and TMF Loading}

\subsubsection{Mechanisms of Fatigue Crack Initiation and Propagation in Superalloys}

As discussed previously, the results of numerous elevated LCF tests conducted in air and in vacuum confirm that oxidation strongly influences fracture mode, and fatigue life. In Ni-base superalloys, surface oxidation occurs generally at a uniform rate at the surface except where discontinuities in alloy composition occur. The grain boundaries in conventionaily cast (CC) superalloys, interdendritic zones for directionally solidified (DS) and single crystal (SX) superalloys, and carbide protrusions at the surface are examples of discontinuities where preferential oxidation occurs. The rate of oxidation at these discontinuities is greater than that of the surrounding matrix and leads to the formation of oxide spikes, as illustrated in Figure 2-9 ${ }^{15}$. Many investigators have shown that oxide spikes act as preferential sites for crack initiation for numerous CC, DS, and SX superalloys $\left(\mathrm{AM}-1^{8}, \mathrm{~B} 1900+\mathrm{Hf}^{43}, \mathrm{NN}-100^{44}, \mathrm{IN}-738^{32,45,46}, \mathrm{Mar}-\mathrm{M} 200^{36}\right.$, Mar-M $246^{47}$, Mar-M24742,48 , Mar-M509 $9^{30,31,49}$, Rene $80^{27,28,50}$, Rene $4^{51}$, Rene $120^{52}, \mathrm{SC} 16^{45}$ ). It has also been observed by a number of investigators that interdendritic micro-porosity, formed during casting of DS and SX superalloys, can also act as fatigue crack initiation sites $^{7,8,45,53}$.

In $\mathrm{CC} \mathrm{Ni}$ and Co-base superalloys, the fatigue crack propagation mode is strongly dependent on the type of fatigue test, i.e. IT-LCF or TMF. For both IT-LCF and OP-TMF loading conditions, fatigue cracks are generally found to propagate transgranularly, usually along interdendritic zones ${ }^{30-35,40,43,49}$. It is also found that fatigue cracks in ITLCF and OP-TMF tests can initially progress intergranularly followed by transgranular 
progression $^{27,32,36,43,47,48,50}$. In IP-TMF tests, the crack propagation path is generally found to be intergranular $35,36,40,42,48$. The observed crack propagation path can be rationalized by considering the different damage mechanisms observed during IP and OP-TMF tests. During IP-TMF loading the maximum tensile strain is applied at maximum temperature, where the cohesive strength of grain boundaries can be lower than the grains ${ }^{43}$, and conditions favor the nucleation and growth of intergranular cavities ${ }^{40}$.

In DS and SX alloys, fatigue cracks are found to progress interdendritically where microporosity, impurities, and carbides are present ${ }^{45,51-53}$. Comparison of fatigue life of CC, DS and SX superalloys demonstrate the superiority of DS and SX superalloys in LCF, TMF, and thermal fatigue (TF) tests ${ }^{7,45,52,54}$. The superiority of DS or SX superalloys can be attributed to: (1) the lack of transverse grain boundaries which removes potential crack nucleation sites ${ }^{52}$, and (2) the lower elastic modulus (E) which results in a lower stress amplitude than $\mathrm{CC}$ superalloys at the same mechanical strain range ${ }^{54}$. This initial attribute (1) has been corroborated by high temperature LCF results with DS Rene 120 loaded at $90^{\circ}$ (transverse loading) to its growth direction ${ }^{52}$. The fatigue crack propagation, under transverse loading was purely intergranular, and the fatigue life was shorter than in all other DS grain orientations.

From the preceding discussion, oxidation and creep damage mechanisms influence both fatigue crack initiation and propagation. The following sub-sections will discuss the role of each damage mechanism in further detail. 


\subsubsection{Creep-Fatigue Interaction}

The first observed effect of an increased creep component in fatigue is usually a transition from transgranular to intergranular fatigue crack initiation ${ }^{25}$. One method for determining the creep component of the inelastic strain range $\left(\Delta \varepsilon_{\text {in }}\right)$ is the Strain Range Partitioning (SRP) method ${ }^{55}$. In this method, the inelastic strain is partitioned into one of four types, $p p, p c, c p$, and $c c$. The $p$ denotes plastic strain while the letter $c$ stands for creep strain, and the first and second letters refer to tensile and compressive halves of the cycle, respectively. A Manson-Coffin type relationship can be determined for each of the individual strain-range components as follows:

$$
\Delta \varepsilon_{x y}=A N_{x y}^{\beta}
$$

where $x y$ denotes one of the four types of inelastic strain, and the symbols $A$ and $\beta$ are constants. The total fatigue life can be determined by combining each of the partitioned inelastic strain range components as follows:

$$
\frac{1}{N_{f}}=\frac{1}{N_{p p}}+\frac{1}{N_{c p}}+\frac{1}{N_{p c}}+\frac{1}{N_{c c}}
$$

In practice, a large amount of experimental data is necessary to use SRP as a life prediction tool ${ }^{56}$. The application of SRP to TMF life prediction is considerably more complicated than isothermal LCF tests; however, the significance of the SRP method, is 
that it can be applied to differentiate between the different types of strain contributing to the fatigue damage process ${ }^{56}$.

The cyclic variation in temperature exerts a strong influence on the deformation (flow) behavior observed in IP and OP-TMF tests. This is manifested in the size and shape of the hysteresis loop, which evolves during TMF cycling. Consider the IP and OP-TMF hysteresis loops illustrated in Figure $2-10^{56}$. The IP hysteresis loop illustrates the development of $\mathrm{cp}$ (tensile creep reversed by compressive plasticity) type deformation since the tensile strain is applied at high temperature, whereas the OP hysteresis loop exhibits pc (tensile plasticity reversed by compressive creep) type deformation. For a number of materials (iron-nickel-base superalloy A286, tantalum alloy T-111, alloy 316 stainless steel), isothermal $\mathrm{cp}$ loading results in intergranular damage evolution by grain boundary sliding, void formation by triple point wedge cracking or grain boundary particle shearing, and from tensile stresses normal to the loading axis ${ }^{57}$. For many materials cp-type deformation is found to be the most detrimental to fatigue life ${ }^{58}$.

Kleinpass et al. $^{40}$ have extensively investigated the evolution of intergranular damage during IP and OP-TMF cycling with IN617 nickel-base alloy. In this study, metallographic sections were checked for creep-cavity formation and intergranular cracks. Two mechanical strain ranges were employed in the study and several maximum cycle temperatures were considered to determine the effects of test duration (fatigue life) and maximum cycle temperature on the evolution of intergranular damage. The results of this study are illustrated in Figure $2-11^{40}$. The results of this investigation indicate that 
specimens subjected to IP-TMF loading exhibited considerably more intergranular damage than OP-TMF loading at the same maximum temperature and mechanical strain range. The extensive intergranular damage observed in IP-TMF specimens was attributed to the high tensile stress imposed at high temperature, which favors nucleation and growth of intergranular cavities.

There is evidence to suggest that intergranular damage is partially reversed during compressive loading in isothermal LCF tests. Type 316 stainless steel $^{57}$ tested under $^{2}$ various hold conditions at $1300^{\circ} \mathrm{F}\left(705^{\circ} \mathrm{C}\right)$ exhibited intergranular cracking when the hold was tensile (cp-type cycle). If both tensile and compressive holds were incorporated (cctype cycle) the fatigue life was longer, and the fracture mode was mixed intergranular and transgranular. It has also been shown by Kleinpass et al. ${ }^{40}$ that the amount of intergranular damage is less severe under IT-LCF cycling than IP-TMF. The later point can be rationalized on the basis that the IP-TMF cycle is not 'balanced' as is the fully reversed isothermal LCF cycle (cc-type). During IP-TMF loading, the compressive loading portion occurs at low temperature where the tensile creep deformation is reversed by plasticity. This results in progressive failure by grain boundary ratcheting effects ${ }^{38}$ involving high-temperature grain boundary sliding in tension, which is not reversed by compressive deformation imposed at lower temperatures, where grain boundary sliding does not occur.

In the forgoing, the detrimental effects of cp-type loading and cc-type loading have been discussed; however, there is considerable evidence to suggest that compressive creep- 
fatigue interaction, i.e. pc type loading, can be more damaging than $\mathrm{cp}$ and cc-type loading $^{19,59}$. The significance of this observation is that the OP-TMF cycle, is pc-type since compressive loading occurs at high temperature. In isothermal creep-fatigue studies with compressive dwells, a considerable tensile mean stress can evolve during the test due to stress relaxation. It has been suggested that the tensile mean stress produced during compressive dwells may enhance crack growth ${ }^{60}$. However, it is expected that fewer voids will grow, compared to cp-type cycling, because of cavity healing under compressive normal stress ${ }^{57}$.

One means of rationalizing the lower pc fatigue life observed in some materials is to consider environmental interaction. It has been shown by Manson et al. ${ }^{57}$ that pc-type loading of $\mathrm{H}-13$ tool steel results in a thicker oxide layer, numerous surface cracks, and lower fatigue life than other loading types (cp, pp, cc). This can be explained by the growth of an oxide layer that is in equilibrium with the compressive loading conditions of the material; on subsequent load reversal the oxide layer cracks and exposes fresh metal to the oxidizing environment ${ }^{57}$. If environmental effects are removed, i.e. testing is conducted in vacuum or inert environment, one would expect to clearly differentiate between the role of creep and oxidation damage for the different TMF cycles. In TMF tests conducted by Neu et al. ${ }^{61}$ with 1070 steel in a helium atmosphere, IP-TMF life was doubled, whereas OP- TMF life was increased by 4 times, at the same mechanical strain range. These results suggest that the reduced life for in-phase loading is due to creep damage $^{61}$, whereas life reduction for out-of-phase loading is due to environmental interaction (fatigue-oxidation interaction). 


\subsubsection{Fatigue-Oxidation Interaction}

The oxidation characteristics of Ni-base superalloys vary considerably. A complete review of the oxidation process, and how it is related to alloy composition, is given by Pettit and Meier ${ }^{62}$. However, some simple generalizations about the oxidation process in $\mathrm{Ni}$-base superalloys can be made. Under static conditions, when a superalloy is initially exposed to a high temperature oxidizing environment, the weight change due to oxidation is generally found to be linear with time (see Figure $2-12^{63}$ ). As this transient oxide layer thickens, the rate of oxidation becomes limited by the diffusion of oxygen and oxide forming metallic elements. The rate at which the oxide layer grows is then found to be proportional to the square root of time, and thus parabolic oxidation kinetics become established (see Figure 2-12 ${ }^{63}$ ). Depending on the specific composition of the superalloy, $\mathrm{Cr}, \mathrm{Al}$, and $\mathrm{Ti}$, or a combination of these elements, are selectively oxidized in the process of forming the oxide layer. The material adjacent to the surface is depleted of these elements and is associated with the formation of a $\gamma^{\prime}$ depleted zone in nickel-base superalloys, as illustrated in Figure 2-13 $3^{64}$. The properties of the $\gamma^{\prime}$ depleted zone are known to be inferior to that of the surrounding matrix. The $\gamma^{\prime}$ depleted zone has been associated with the concentration of plastic deformation leading to microcracking and fatigue failure ${ }^{25,42}$.

The amount of oxygen uptake will be higher for IT-LCF tests conducted at the maximum TMF cycle temperature than observed during TMF tests of the same duration ${ }^{37}$. If oxidation damage is the major mechanism in controlling LCF life, then one would expect that LCF tests at the maximum TMF test temperature will result in conservative life 
predictions. As discussed previously, IP and OP-TMF lives are generally found to be considerably different (usually lower) than IT-LCF tests conducted at the maximum TMF test temperature. Thus, one may postulate that cyclic loading, in combination with transient thermal conditions, must have a significant effect on oxidation behavior.

A number of investigators have reported fatigue-oxidation interaction in IT-LCF and TMF tests $31,51,65,66,67$. In general, these investigators have observed that fatigue accelerates the oxidation process and that the oxide scale thickness is dependent on the inelastic strain range. Higher inelastic strain ranges result in a thicker oxide scale, despite having a shorter fatigue life. From a physical basis these observations can be explained by considering the mechanical properties of the oxide layer. Referring to Figure $2-14^{68}$, which illustrates the strain to fracture of a chromia oxide layer formed on Nimonic 75 , one can see that the fracture strain is quite small $\left(<10^{-4} \mathrm{~mm} / \mathrm{mm}\right)$, and that it decreases with temperature. The oxide layer experiences a mechanical strain arising from, (1) internal stresses that build up during oxide layer growth ${ }^{48},(2)$ geometrical factors ${ }^{48,69},(3)$ internal stresses caused by substrate-oxide thermal expansion mismatch ${ }^{48,70}$, and (4) the applied mechanical strain. The cumulative contribution of mechanical strain arising from each of these sources can cause the oxide layer to fracture under tensile loading and/or buckle during compressive loading causing spalling and scallop formation (see Figure $\left.2-15^{71}\right)$. When the oxide layer is damaged during cyclic loading, fresh metal is exposed to the environment, which is rapidly oxidized. The repeated fracture of the oxide layer and the oxidation of exposed metal, results in a thicker oxide layer than would be observed under static oxidation conditions. 
The repetitive process of oxide fracture and oxidation of the exposed metal is detrimental, since this channels oxide growth into the substrate ${ }^{51}$, which can nucleate fatigue cracks. As these fatigue cracks grow, the material ahead of the crack tip is changed by oxidation ${ }^{72}$. These changes include the formation of an oxide layer ahead of an advancing crack, which can also be accompanied by the formation of a $\gamma^{\prime}$ depleted zone ahead of the oxide layer. The degree to which oxxidation will affect crack propagation rates and propagation mode is dependent on factors such as, strain range, strain rate, temperature range, and deformation path (IP or OP-TMF, etc.). For example, the OP-TMF cycle will be especially detrimental because the oxide layer is in tension at low temperature when the oxide layer has insufficient ductili:ty to prevent fracture ${ }^{48}$. When all other factors are considered equal, strain range has a profound effect on the dominant damage mechanism. At low strain ranges (longer life) when oxidation rates exceed the crack growth rate, oxidation damage is the dominant damage mechanism ${ }^{42}$. At higher strain ranges, the crack growth rate can exceed the oxidantion rates ${ }^{48}$, resulting in the oxide layer trailing the crack tip. Therefore, at higher strain : ranges fatigue is generally the dominant damage mechanism.

A number of investigators have studied the micro-mechanisms of fatigue crack growth with environmental interaction ${ }^{28-31,42,48,50,74,75}$. Two mechanisms have been proposed in the literature. The first mechanism involves the successive fracture of oxide spikes due to oxidation embrittlement of the materiasl ahead of an advancing fatigue crack (see Figure $2-16^{33}$ ). As the fatigue crack opens in tension, total or partial fracture of the oxide spike is accompanied by oxide advance ahead of the crack tip. The kinetics of the oxidation are 
found to be higher ahead of the advancing crack than in the bulk due to a reversion to transient (i.e. linear) oxide kinetics ${ }^{63}$, and to dislocation networks acting as short-circuit diffusion sites $^{73}$. This mechanism is supported by a number of researchers ${ }^{28-31,50}$.

The second mechanism involves the successive growth of the oxide layer ahead of an advancing fatigue crack, until it reaches some critical thickness and ruptures. There are two types of oxide intrusions associated with this fatigue-oxidation damage mechanism ${ }^{74,75}$. The first type of oxide intrusion is referred to as 'Type $I$ ' and is characterized by the formation of a continuous oxide layer with no stratification. In Type I oxide growth, the continuous oxide layer is cracked when it reaches a critical thickness, exposing fresh metal to the oxidizing environment, where the process repeats itself as illustrated in Figure 2-17 (A $)^{74}$. The second type of oxide intrusion is referred to as 'Type II' and is characterized by a stratified oxide layer. Type II oxide intrusion is similar to Type I in that the oxide layer reaches some critical thickness and ruptures; however, when the oxide ruptures, it detaches from the surface. When the oxide layer detaches, a larger area is exposed to the oxidizing environment, and the process repeats, as illustrated in Figure 2-17 $(B)^{74}$. In experiments with Mar-M247 nickel-base superalloy, Sehitoglu and Boismier ${ }^{48}$ assumed the critical rupture value of the oxide layer to be the sum of the crack tip oxide thickness and the $\gamma^{\prime}$ depleted zone. The actual type of oxide intrusion that is observed is dependent on the alloy, temperature range, type of cycling (IT-LCF, OP or IP-TMF) and the strain range $e^{42,48,74,75}$. A combination of the two oxide intrusion types may also be prevalent under the same testing condition ${ }^{42,48,74,75}$. 
In the forgoing it has been shown that oxidation has a deleterious effect due to oxide embrittlement, and localized failure of the oxide layer. One additional effect of oxidation on crack growth has not been considered. Oxidation along the crack faces is associated with a volume expansion, which can result in fatigue crack blunting due to an oxide wedging effect ${ }^{72}$. The oxide wedging mechanism is shown schematically in Figure $2-18^{72}$. A number of investigators ${ }^{27,40,72}$ have reported that the oxide wedging mechanism can slow or stop the propagation of a fatigue cracks.

\subsection{Microstructural Changes and Cyclic Response Under TMF and LCF Cycling}

As mentioned previously, the cyclic stress-strain response is not symmetrical during TMF loading because of changes in flow stress and elastic modulus between the two temperature extremes used in a TMF test. Typically, IP cycling results in compressive mean stress, and OP cycling results in tensile mean stress. The cyclic hardening-softening behavior on the other hand, is generally different during TMF loading than that observed for IT-LCF cycling conditions. For example, TMF tests conducted by Castelli et al. ${ }^{76}$ on Hastelloy $\mathrm{X}$ (a solid solution strengthened superalloy exhibiting strong dynamic strain aging characteristics), illustrate that the cyclic hardening behavior within the dynamic strain aging temperature regime for this material, is not 'bounded' by isothermal LCF

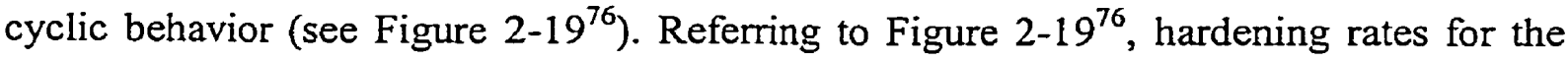
compressive and tensile peak stress amplitudes are higher at $400^{\circ} \mathrm{C}$ (the lower TMF cycle temperature) than during IT-LCF cycling at $400^{\circ} \mathrm{C}$. In this case, the different cyclic behavior was due to microstructural changes that occurred during the high temperature portion of the TMF cycle $\left(600^{\circ} \mathrm{C}\right)$. Fine $\mathrm{M}_{23} \mathrm{C}_{6}$ carbides were precipitated during the high temperature portion of the TMF cycle, whereas carbide precipitation did not occur in 
LCF tests until temperatures exceeded $500^{\circ} \mathrm{C}$. As a result, loading at low temperature $\left(400^{\circ}\right)$ during the TMF cycle occurred when the microstructure was significantly different than observed in IT-LCF tests conducted at $400^{\circ} \mathrm{C}$.

Marchand et al. ${ }^{43}$ has investigated the effects of TMF cycling on the microstructural features of B $1900+$ Hf nickel-base superalloy. In this study $\gamma^{\prime}$ coarsening was observed in all test conditions and was more pronounced for IP cycling than OP or IT-LCF cycling. The dislocation density was also found to be higher under IP cycling conditions than OP or IT-LCF cycling. It was also observed that the tensile flow stress increased during IP and OP-TMF tests (cyclic hardening), whereas the compressive cyclic flow stresses between $\mathrm{P}$ and OP-TMF loading conditions were found to be radically different. OPTMF loading resulted in very pronounced softening of the compressive flow stress, whereas IP tests exhibited a relatively stable cyclic compressive flow stress. The increase in tensile cyclic flow stress in IP tests was attributed to dislocation networks surrounding the $\gamma^{\prime}$, whereas the pronounced softening behavior in OP tests resulted from the combination of dislocation network strengthening, and the interaction of the directional strain field surrounding the $\gamma^{\prime}$ precipitates ${ }^{43}$.

From the preceding discussion, the observed differences between cyclic hardeningsoftening response are due to a number of different deformation mechanisms that can be active during transient thermal and mechanical loading. The cyclic hardening-softening behavior is also dependent on the material being tested. In general, at higher temperatures a more homogeneous dislocation distribution results from the increased ease of thermally 
assisted climb and cross-slip ${ }^{25}$. At the low temperature portion of a TMF cycle, $\gamma^{\prime}$ particles must deform by planar shearing by paired dislocations, or a dislocation must bypass $\gamma^{\prime}$ particles by means of a looping mechanism ${ }^{36}$. This will be strongly dependent on the composition of the superalloy, e.g. superalloys with high volume fraction of $\gamma^{\prime}$ $(>50 \%), \gamma^{\prime}$ shearing is the primary strengthening mechansism ${ }^{43}$. The interaction between the high and low temperature deformation mechanisms can result in increased mobile dislocation density at high temperature ${ }^{36}$ and microstructural changes that would not normally be observed during isothermal cycling.

\subsection{Summary}

The preceding discussion highlights the significant differences in damage mechanisms and material behavior observed under TMF and LCF cycling conditions. A number of general observations can be made about the behavior of Ni-base superalloys during TMF and LCF cycling conditions:

1. IT-LCF tests at the maximum cycle temperature used in TMF tests, in many cases, do not result in a conservative estimate of fatigue life,

2. Deformation path (IP or OP-TMF cycling) exerts a strong influence on the observed damage mechanisms and material behavior, and

3. The damage mechanisms and cyclic behavior during TMF cycling are poorly captured by isothermal testing conditions. 


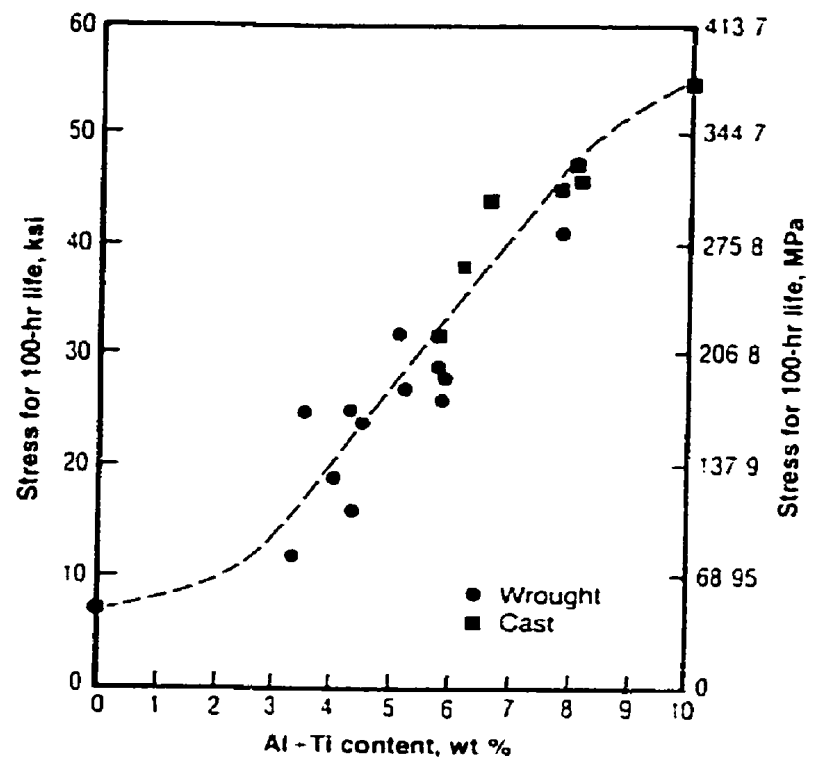

Figure 2-1 - Effect of $\mathrm{Al}+\mathrm{Ti}$ content on creep rupture strength of nickel-base superalloys at $870^{\circ} \mathrm{C}^{15}$.

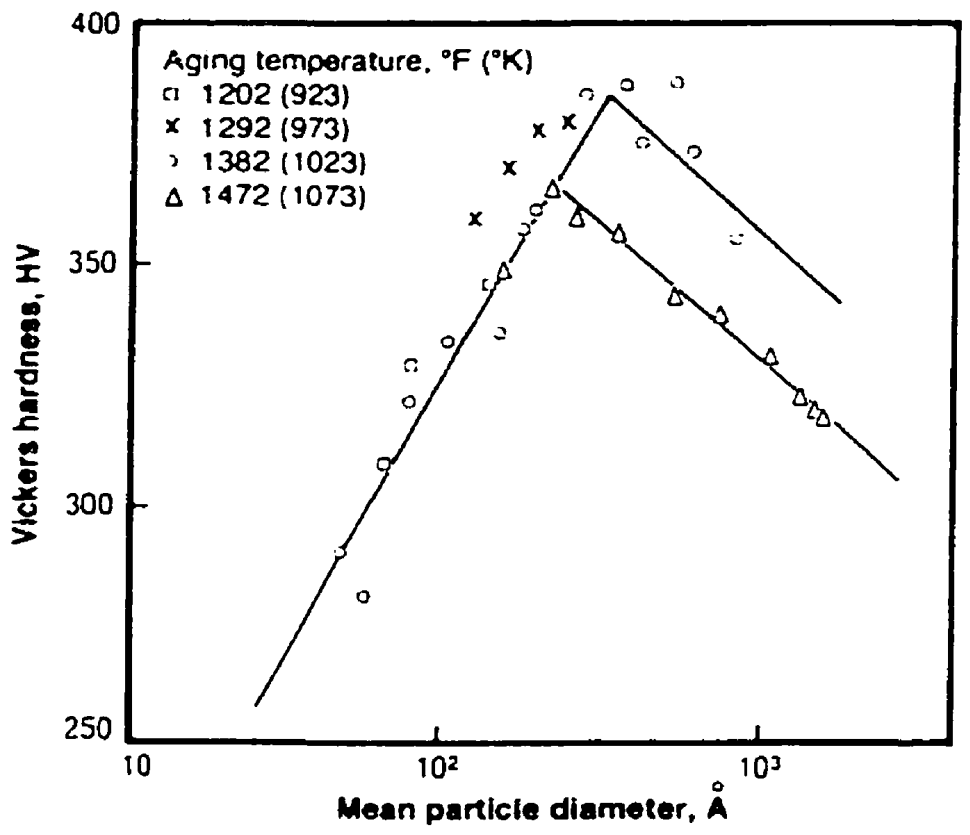

Figure 2-2 - Strength (hardness) versus $\gamma^{\prime}$ precipitate diameter in a nickel-base superalloy ${ }^{15}$. 


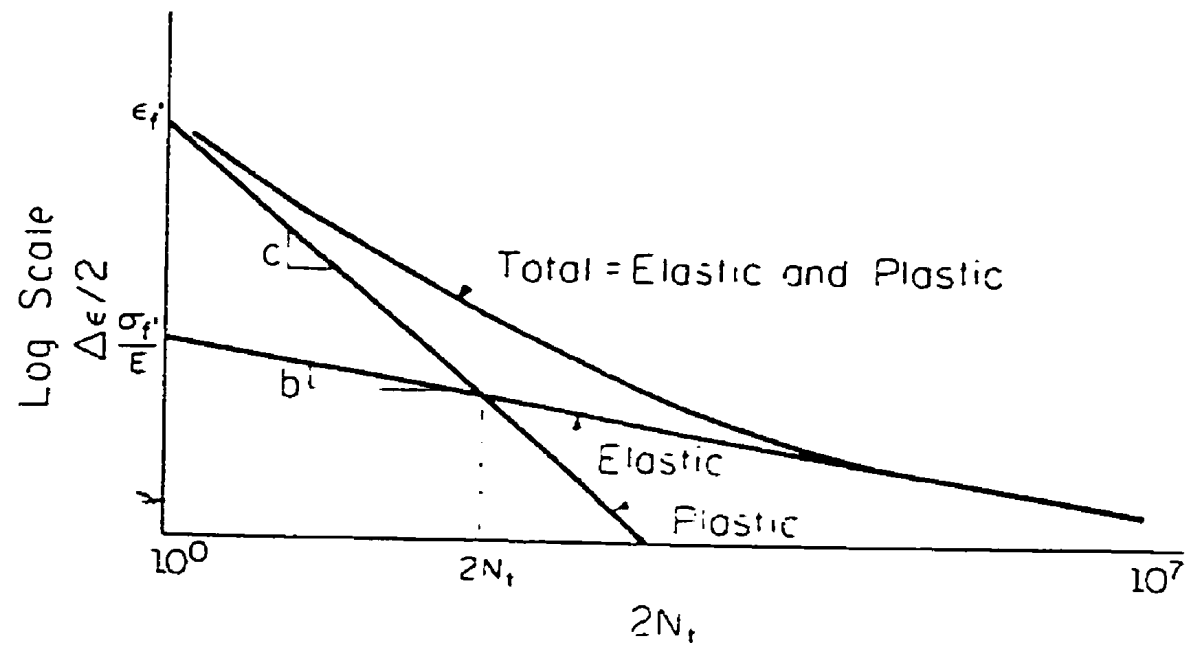

Figure 2-3 - Total strain range as sum of elastic and inelastic strain range components versus life plotted using logarithmic coordinates ${ }^{18}$.

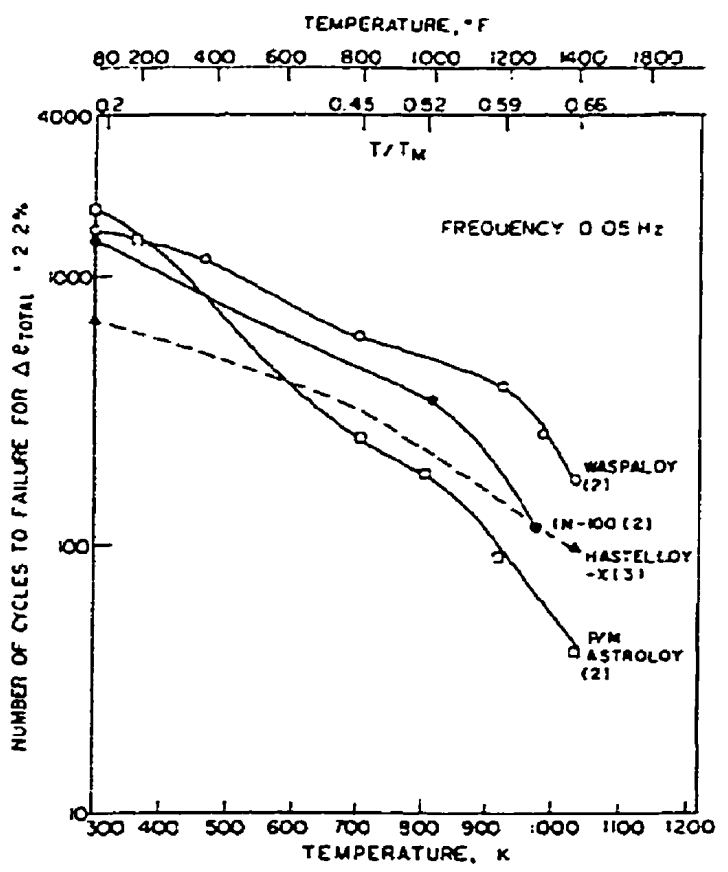

Figure 2-4 - Variation in number of cycles to failure with temperature for several nickelbase superalloys tested at a total constant strain range of $2.2 \%{ }^{23}$. 


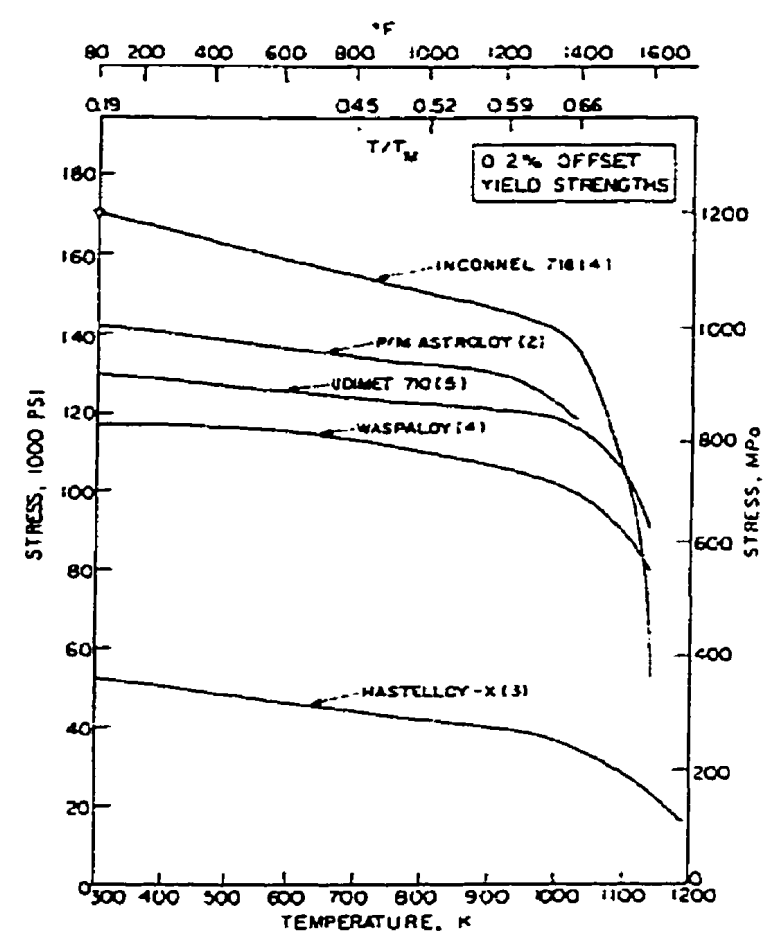

Figure 2-5 -0.2 percent offset flow stress as a function of temperature for several nickelbase superalloys ${ }^{23}$.

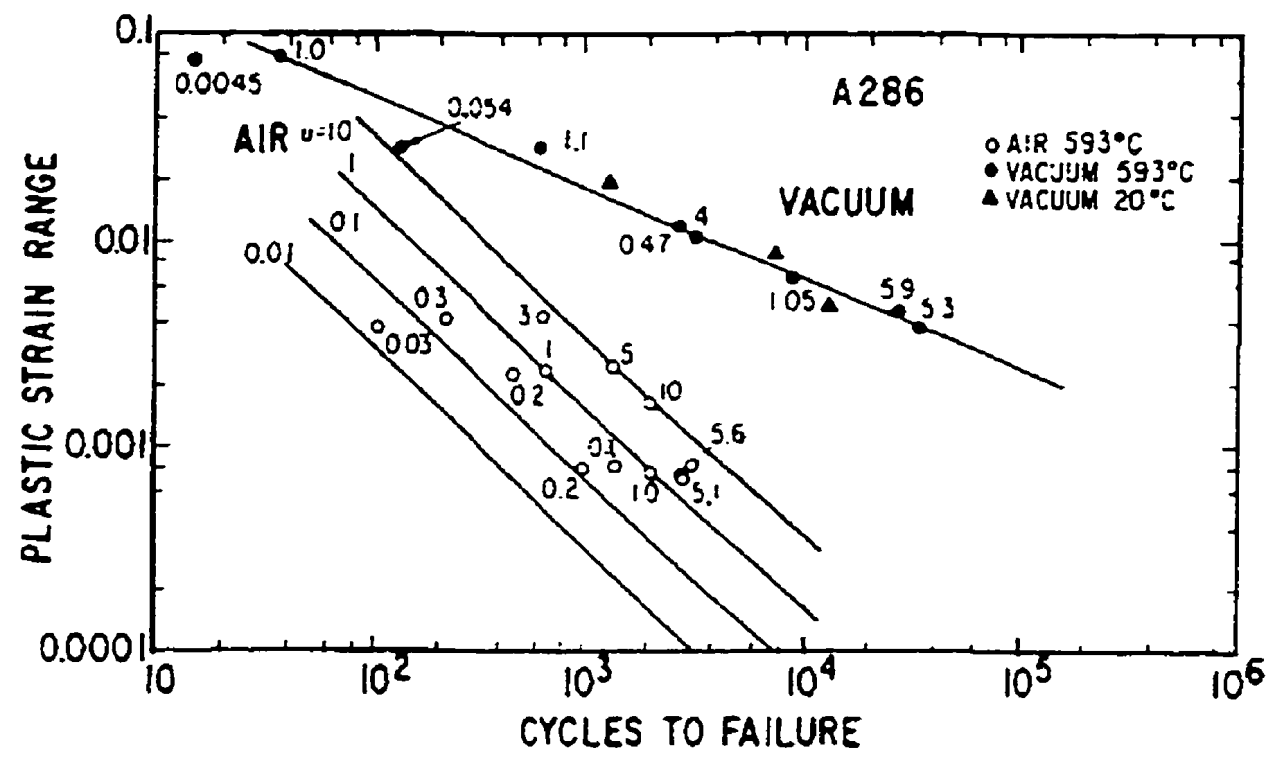

Figure 2-6 - Plastic strain range versus fatigue life for A286 in air and vacuum at $593^{\circ} \mathrm{C}$. Numbers adjacent to test points indicate frequency in cycles per minute $(\mathrm{cpm})^{22}$. 

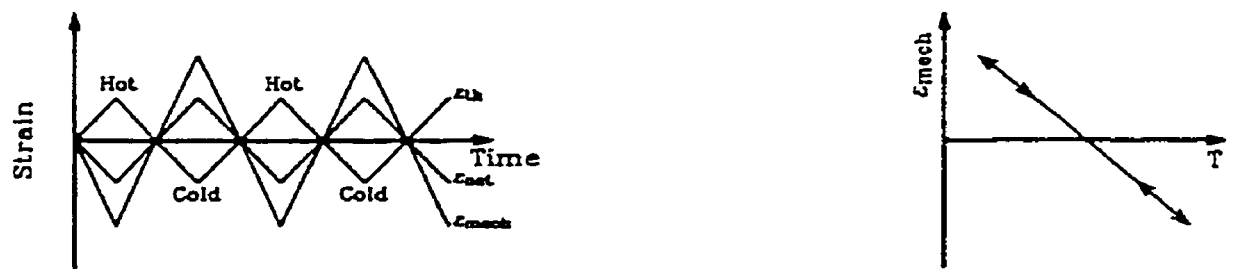

a) Out-of Phase (OP)
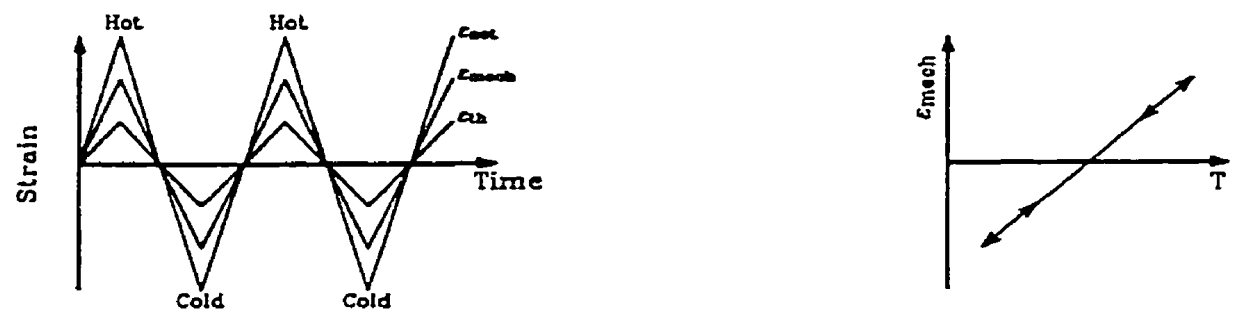

b) In-Phase (IP)
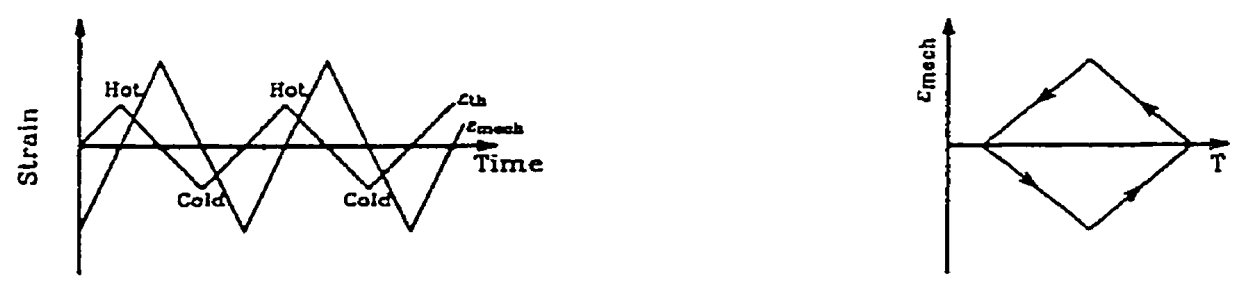

c) Counterclockwise Diamond (CCWD)

Figure 2-7 - Strain versus time, and strain versus temperature relationships for OP, IP and CCWD-TMF cycles ${ }^{33}$. 


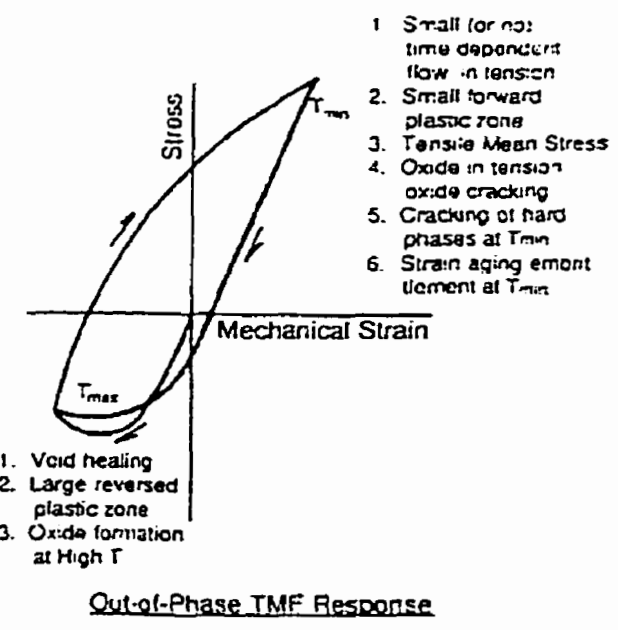

Fig- 2(a) Strece-mochanical straln response and cortain mochanisme Tor OP TMF cese

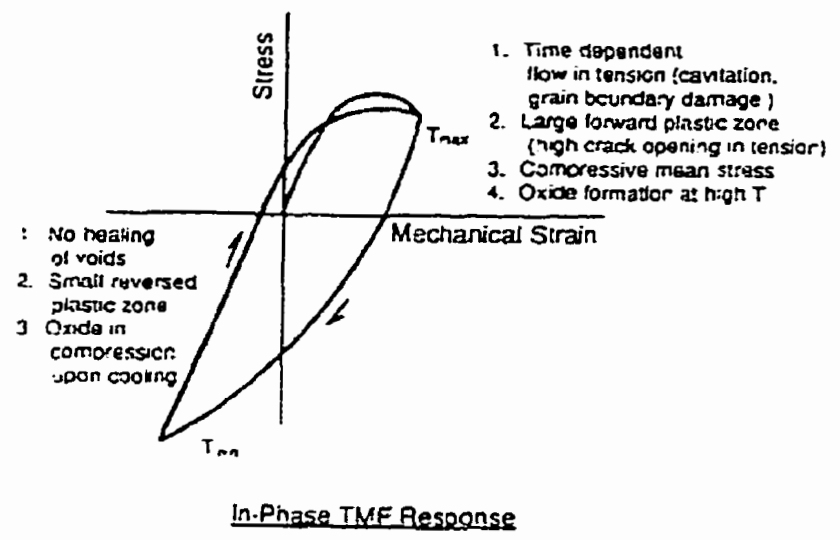

Figure 2-8 - Stress-mechanical strain response and damage mechanisms operative under in-phase and out-of-phase thermal-mechanical fatigue cycles ${ }^{42}$. 


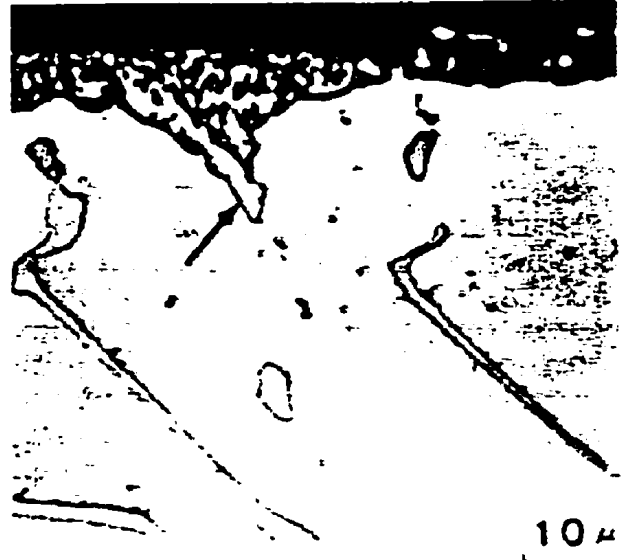

(a)

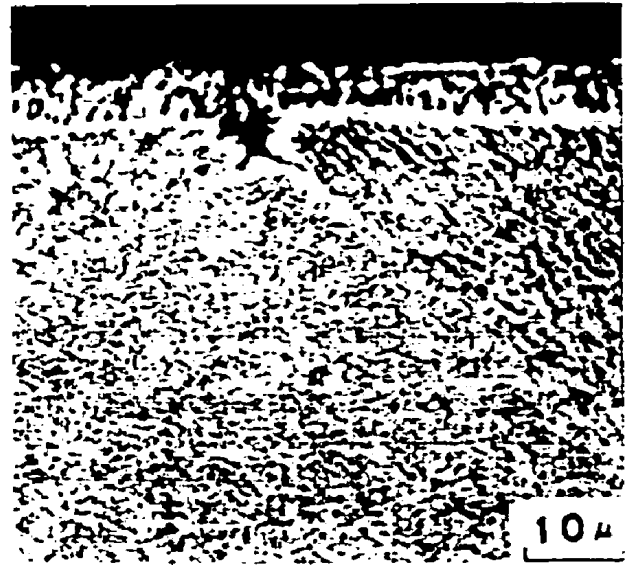

(b)

Figure 2-9 - (a) Accelerated oxidation of MC carbide (arrow) at surface of Mar-M200 at $925^{\circ} \mathrm{C}$. (b) Accelerated oxidation of grain boundary in Udimet 700 at $760^{\circ} \mathrm{C}^{15}$.
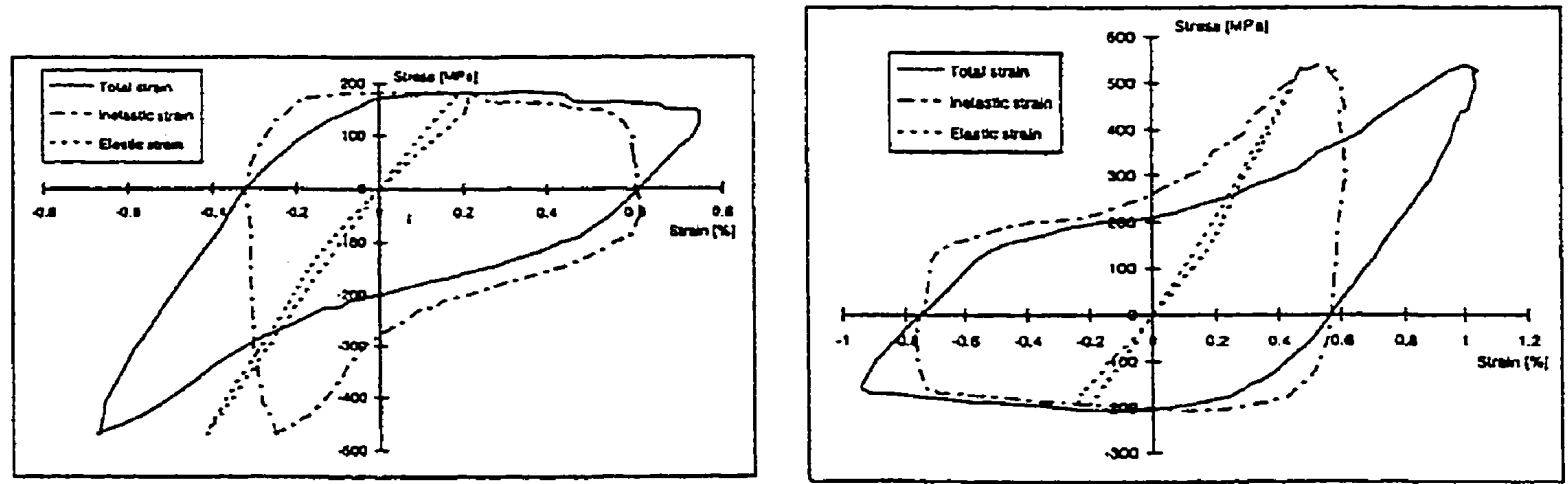

Figure 2-10 - Stress-mechanical strain hysteresis loops for an in-phase (left) and out-ofphase (right) TMF test ${ }^{56}$. 

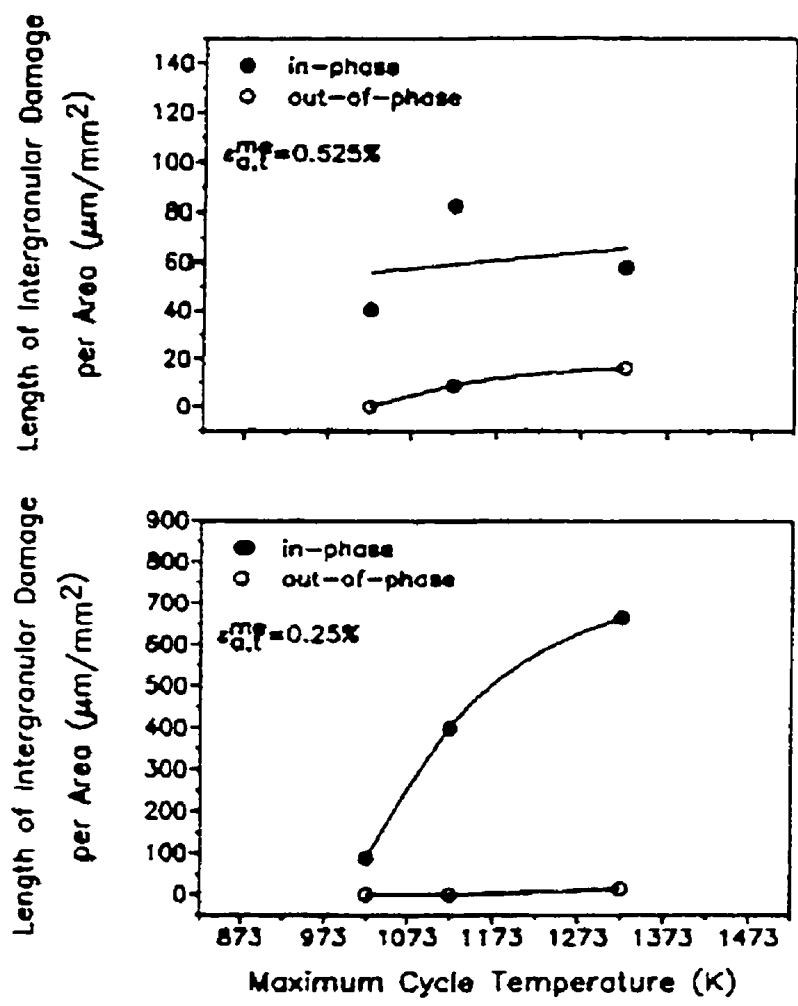

Figure 2-11 - Influence of phase relation and maximum cycle temperature on the development of intergranular damage ${ }^{40}$.

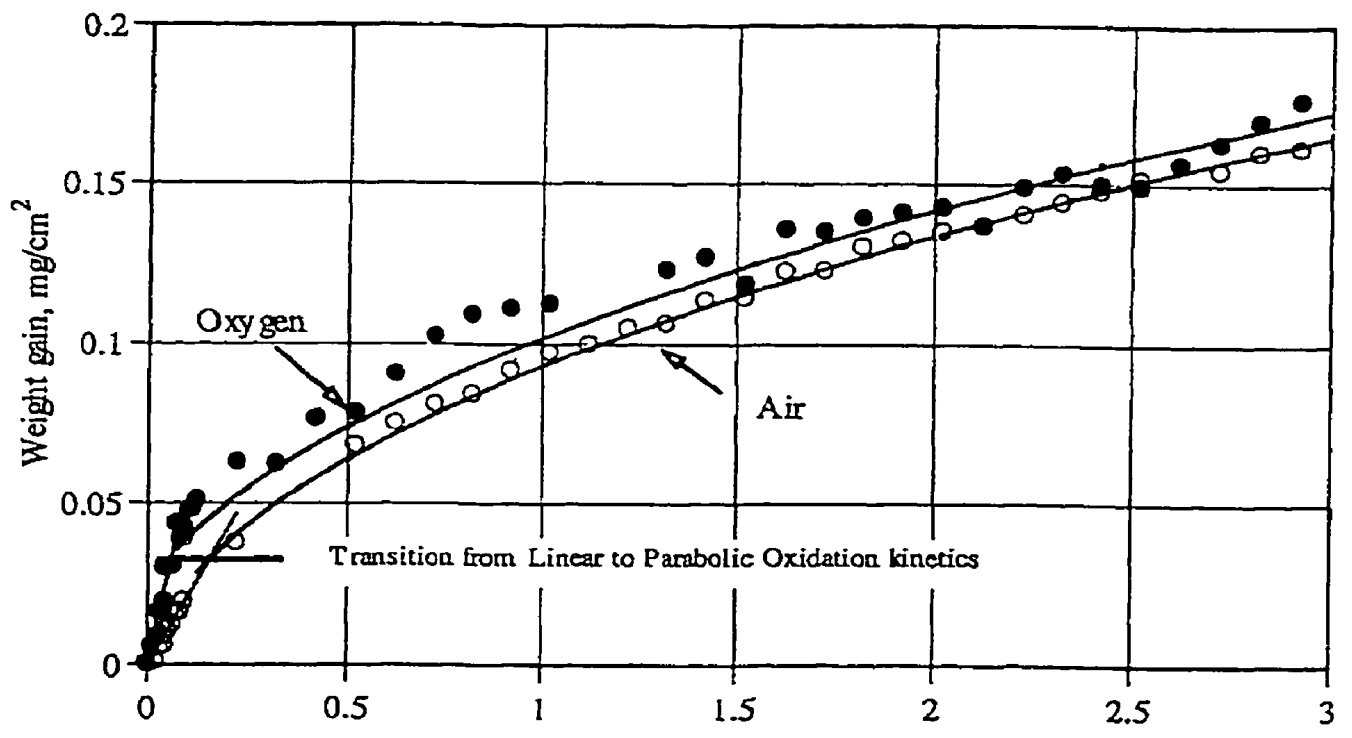

Time at $1200^{\circ} \mathrm{C}$, Hours

Figure 2-12 - Effect of oxygen activity on transient and steady state oxidation of CMSX3 at $1200^{\circ} \mathrm{C}^{63}$. 

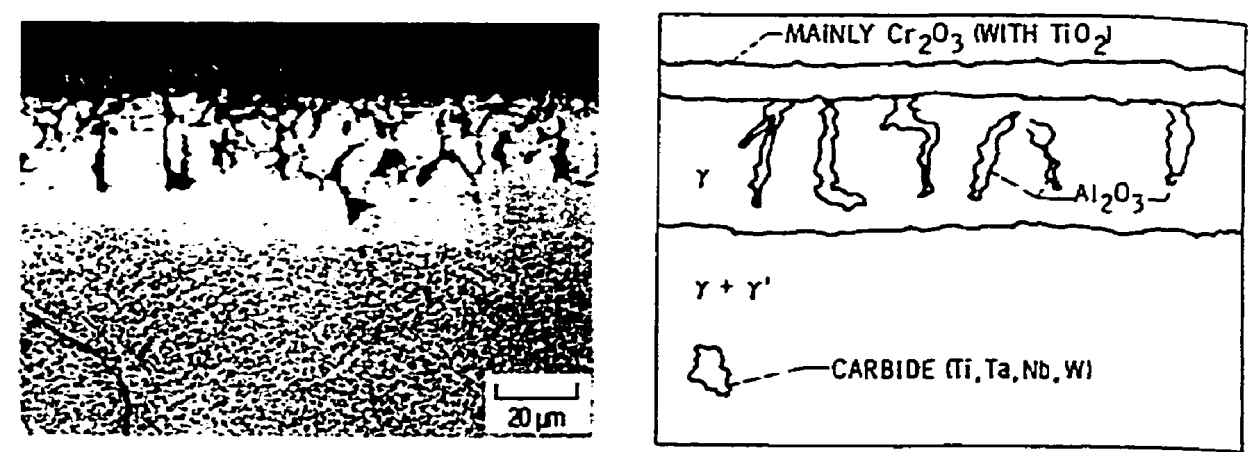

Figure 2-13 - Microstructure of $\mathrm{IN}-738$ after $24 \mathrm{~h}$ oxidation at $975^{\circ} \mathrm{C}$ showing Ti-rich $\mathrm{Cr}_{2} \mathrm{O}_{3}$ scale, internal oxidation, and $\gamma^{\prime}$ depleted zone ${ }^{64}$.

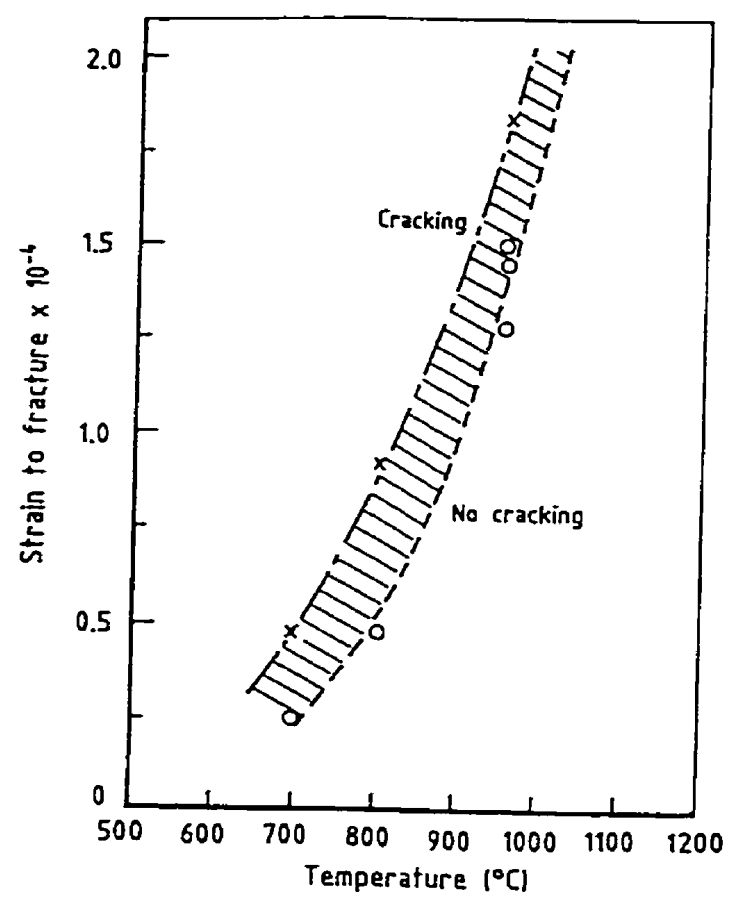

Figure 2-14 - The effect of temperature on the fracture strains of chromium oxides formed on Nimonic $75^{68}$. 


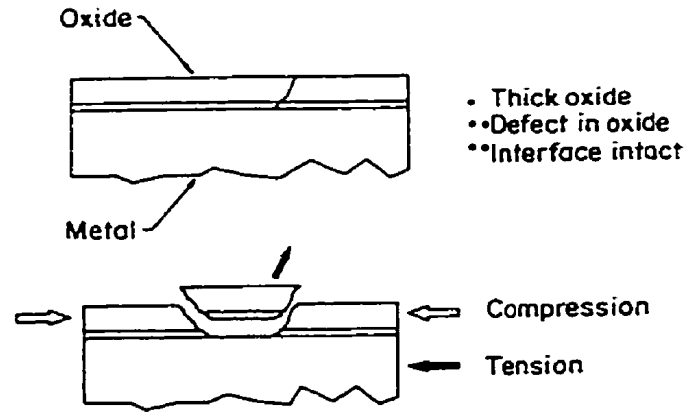

a)

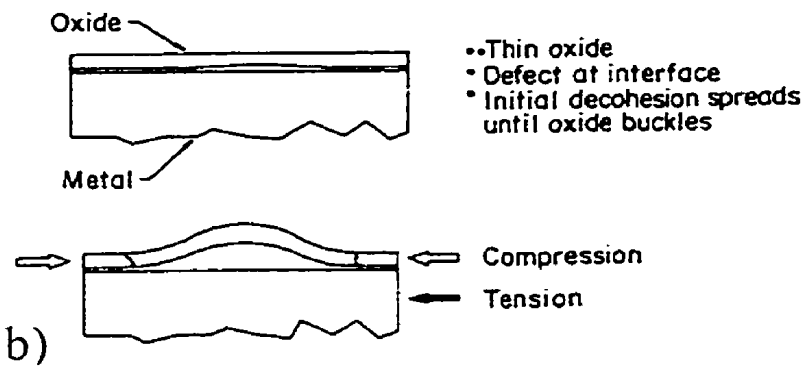

Figure 2-15 - Mechanisms of scalloping: (a) mode 1; (b) mode $2^{71}$. 


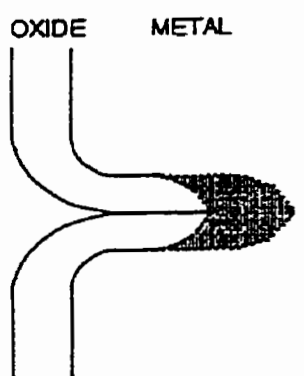

(a)

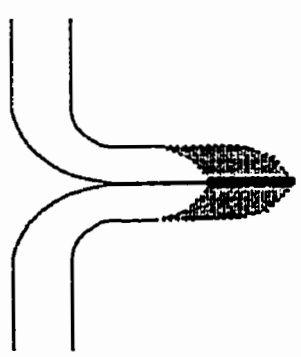

(b)

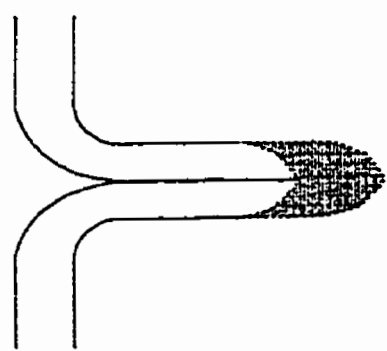

(c)

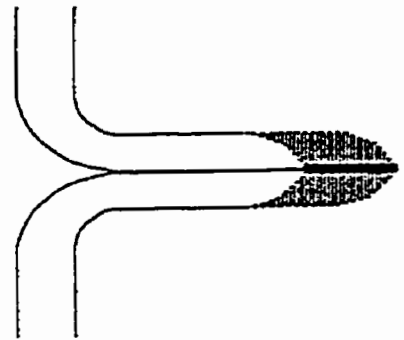

(d)

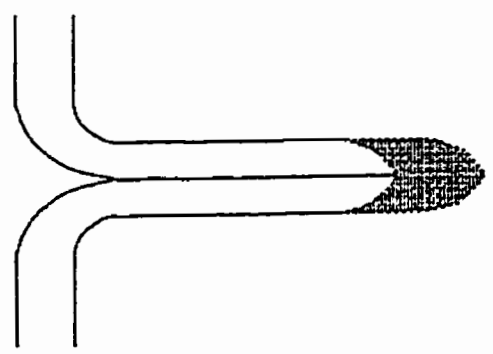

(e)

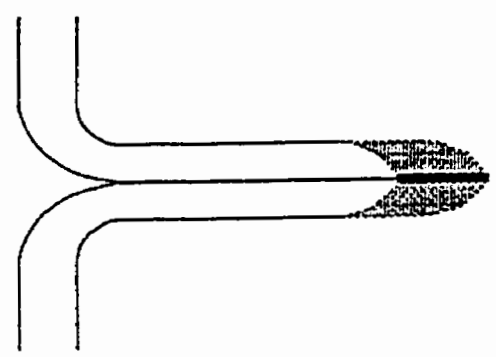

(t)

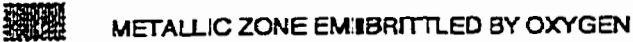
CRACK IN THE METEL

Figure 2-16 - Fatigue-oxidation crack g:rowth mechanism by embrittled oxide spike fracture. a) oxygen diffuses into the metal $\vec{u}$ front of the oxide spike tip; $b$ ) the embrittled metallic zone fractures; c) the fresh fracturred metallic surfaces oxidizes and the oxygen diffuses into the metal again; process repeants as sketched in d), e) and $f)^{33}$. 


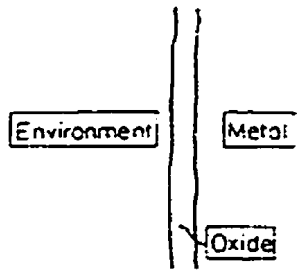

(a)

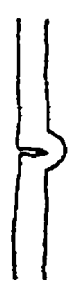

(c)

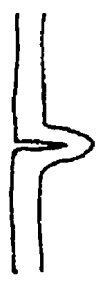

A)

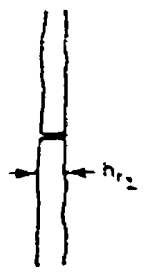

(b)

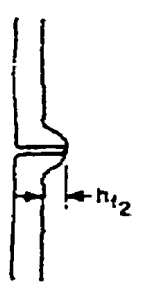

(d)

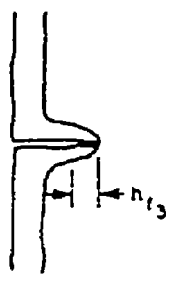

(f)

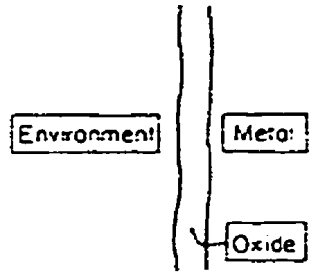

(a)

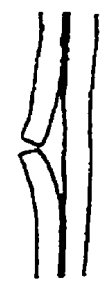

(c)

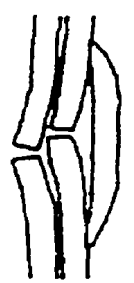

(e)

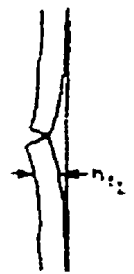

(b)

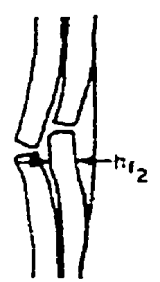

(d)

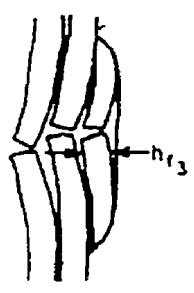

(j)

Figure 2-17 - Fatigue-oxidation crack growth mechanism by oxide intrusion of Type I (A) and Type $\Pi$ (B) after Sehitoglu et al. ${ }^{74}$. The oxide intrusion mechanism process occurs by the following steps: a) uniform oxidation layer forms on the surface; b) when the oxide reaches some critical thickness, $h_{f}$, the oxide ruptures and crack nucleation occurs; c) the fresh metallic surface is exposed to the environment and oxidizes quickly; d) when the oxide reaches some critical thickness, $h_{f 2}$, the oxide again ruptures. The process and repeats as shown in e) and f). 


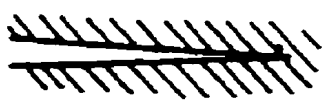

a)

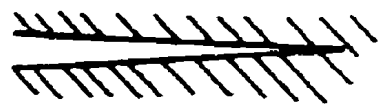

c)

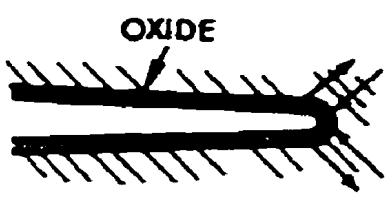

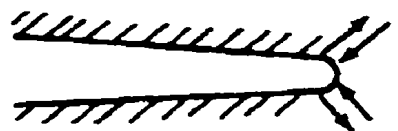

b)

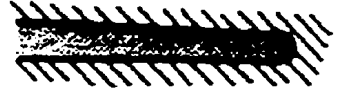

d)

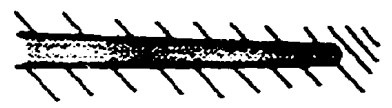

ก

Figure 2-18 - Plasticity $(a, b, c)$ and oxide induced (d, e, f) crack tip blunting ${ }^{72}$.

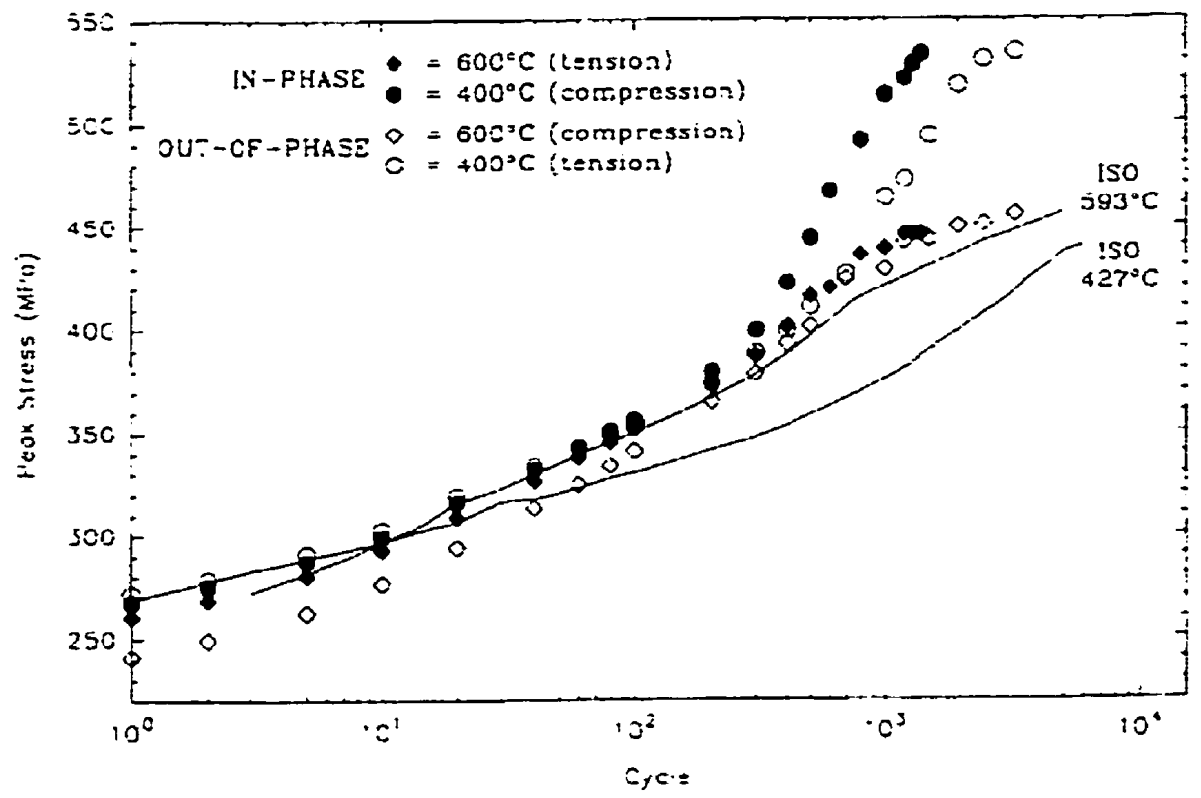

Figure 2-19 - Peak stress versus cycle plot for $400^{\circ} \mathrm{C}$ to $600^{\circ} \mathrm{C}$ in-phase and out-of-phase TMF tests and bounding isothermal tests ${ }^{76}$. 


\section{Review of Thermal-Mechanical Fatigue Testing Methods}

\subsection{Introduction to TMF Testing}

The purpose of TMF testing is to study the damage mechanisms during varying temperature-strain histories. In this type of test, strain (or stress) and temperature are both independently controlled variables. The temperature and strain waveforms both have the same period, but by altering the phase and/or rate of the applied temperature and strain, one can simulate a wide variety of temperature-strain relationships. The period of the TMF cycle is usually longer than the thermal transients in actual components because of limitations with the heating and cooling rates; however, it is possible to faithfully simulate the temperature-strain history of real components.

As mentioned previously, TMF testing utilizes external constraint for controlling the mechanical deformation of the specimen. One method for introducing external constraint is the two-bar structure used by Sehitoglu ${ }^{77}$. A schematic representation of the two-bar structure is illustrated in Figure $3-1^{77}$. Referring to Figure $3-1^{77}$, a bar with a large cross section (bar 2) is utilized to constrain the free thermal expansion of the test coupon (represented by bar 1). A total constraint is obtained when the constraining bar is held at the minimum temperature for the TMF test resulting in an out-of-phase temperaturestrain relationship. An in-phase temperature-strain relationship is obtained when the temperature of the constraining bar and test coupon are varied simultaneously. In this test, total strain is controlled by alternative heating and cooling the elements of the two- 
bar structure, therefore limiting the number of temperature-strain relationships that can be simulated.

The second type of TMF system uses two independent control loops; one for closed loop strain control, the other for closed loop temperature control. In this type of system, the external constraining forces are applied through the grip ends of a uniaxial fatigue specimen by a servo-controlled fatigue machine, while the specimen is uniformly heated and cooled. Both the temperature and strain controlling waveforms can be independently pre-programmed to follow one of the temperature-strain relationships described previously (see Figure $2-7^{33}$ ), or an arbitrary temperature-strain relationship. The control and synchronization of the temperature and strain control loops is facilitated by the use of a computer, which can also be used for data acquisition during a test. Several computerized TMF testing systems have been developed based on this approach $6,34,78,79$.

Despite pioneering work in TMF testing field as early as $1970^{80}$, methods for TMF testing have not been formally standardized by ASTM, or other standards organizations. Therefore, experimentalists frequently resort to various aspects of isothermal LCF testing standards for guidelines in performing TMF tests ${ }^{81}$. Unfortunately, these testing standards do not address issues such as: (1) temperature-strain phasing accuracy, (2) control of temperature under dynamic conditions, and (3) thermal strain compensation. Therefore, the potential for variability in testing methods can compromise the accuracy and reliability of TMF deformation and life data. This, in fact, has been observed in an 
interlaboratory test program for $\operatorname{COST} 501^{\gamma}$ Round $2^{81}$. Initially each participant in the COST consortium used different bar stock and specimen geometries (flat, solid cylinder, and tubular). The open symbols on Figure $3-2^{81}$ represent the results from the initial series of tests in the interlaboratory test program; the scatter in fatigue life is approximately 10 times. When the participants repeated the same tests with specimens from the same bar stock and geometry the fatigue life scatter was reduced to approximately 3.5 times (closed/solid symbols on Figure $3-2^{81}$ ). It is believed that subtle differences in experimental techniques amongst the laboratories involved in this interlaboratory test program have contributed to the considerable variability in life data ${ }^{81}$. Presently, the standardization of TMF testing is in progress; the proposed standard will be referred to, when appropriate, during the description of TMF testing methods within this chapter.

The important aspects for TMF testing are summarized within the following sections. These include a review of stress and strain controlled tests, thermal strain compensation methods, methods for specimen heating and cooling, TMF specimen geometry, data acquisition, and special considerations for analysis of TMF deformation and life data.

\subsection{Stress-Controlled and Strain-Controlled TMF Testing}

The stress-controlled TMF test is technically less challenging to implement than strain controlled testing because no compensation for free thermal expansion and contraction of the test specimen is required. When cycling between fixed stress limits, with significant

\footnotetext{
${ }^{\gamma}$ European collaborative activity related to materials for gas turbines designated as COST
} 
plastic deformation, cyclic softening or hardening behavior is evidenced by a progressive increase or decrease in inelastic strain range, referred to as strain ratcheting. Therefore, stress-controlled testing should be limited to long-life tests exhibiting little plasticity ${ }^{82}$. In specific instances, it is appropriate to use stress-controlled TMF cycling. For example, stress-controlled TMF testing was used to assess the fatigue life for a gas turbine centrifugal compressor ${ }^{83}$, since the mechanical loads are more dominant than thermal loads in this component. Stress-controlled TMF testing has also been used for brittle materials, such as the molybdenum alloy $\mathrm{TZM}^{84}$.

The majority of IT-LCF and TMF tests are conducted under fixed strain limits because this more closely represents the conditions that develop when mechanical strain is induced by thermal cycling ${ }^{21}$. Both axial and diametral strain-control have been used for TMF testing. Diametral strain-control is primarily associated with large strain ranges (greater than $2 \%$ ), because of the higher stiffness attained by using hourglass geometry specimens. Although diametral strain-control has been used in IT LCF and TMF testing, it should be noted that axial strain is the controlled variable. Therefore it is necessary to compute the axial strain from the measured diametral strain. Axial strain can be computed from the following equation given by Berling and $\operatorname{Slot}^{85}$ :

$$
\left.\Delta \varepsilon=\left(1-2 v_{e}\right) \frac{\Delta P}{E A}-2 \Delta \varepsilon_{d} \quad \text { (assuming } \nu_{\mathrm{p}}=0.5\right)
$$

where $\Delta \varepsilon$ is the axial strain range, $\Delta \varepsilon_{\mathrm{d}}$ is the diametral strain range, $\Delta \mathrm{P}$ is the load range, $\nu_{e}$ is the elastic Poisson's ratio, $\nu_{p}$ is the inelastic (plastic) Poisson's ratio, $E$ is the 
modulus of elasticity, and $\mathrm{A}$ is the nominal cross sectional area of the specimen. Referring to Equation (3-1), it is sapparent that controlling diametral strain under LCF and TMF is compounded by a numbe:s of factors:

1. High temperature oxidation on the test specimen is perceived as a ratcheting strain as the oxide layer evolves at elewated temperatures ${ }^{86}$.

2. Materials with a large grain size or preferred orientation tend to be anisotropic, and require special methods of strrain measurement and interpretation, since the value for Poisson ratio changes with orientation of the extensometer with respect to the crystallographic orientation 22,282 .

3. Many materials exhibit cyclic softening or hardening in strain controlled tests, which is accompanied by a redistribution of the elastic and plastic strains. When cycling between fixed diametral strain limits, the axial strain range will increase if the material cyclically hardens, and decrease if the material cyclically softens.

4. Dwell or hold periods in dianmetral strain controlled tests at elevated temperatures frequently cause stress relaxa:tion. Stress relaxation causes a redistribution of elastic strain to plastic strain, and by holding diametral strain constant one will not obtain a constant hold for axial strain.

5. During thermal cycling in a TMF test, the value for the elastic modulus changes as a function of temperature, comprounding axial strain computation and control.

Side contact, axial extensometry ins used by the bulk of TMF testing facilities ${ }^{87}$ for total axial strain control. 
Halford, et al. ${ }^{88}$ have introduced an alternative testing method, referred to as bithermal fatigue. The intended purpose of this test is to introduce many of the damage mechanisms observed in TMF, without the complexity of performing actual TMF tests. In this test, tensile and compressive half-cycles of deformation are applied to the specimen under isothermal conditions, at two distinctly different temperatures. During temperature transients between the two temperatures, the test control unit is in force control to prevent loads from being applied to the specimen. Figure $3-3^{88}$ compares the IP and OP bithermal fatigue cycles to the equivalent TMF cycles. The primary advantages of bithermal fatigue testing are:

1. Compensation for thermal expansion and contraction is simplified over TMF testing since the thermal strain is constant at each temperature in which deformation is being applied, and

2. Temperature uniformity within the gage section is easier to achieve under static temperature conditions than under transient thermal cycling ${ }^{89}$.

A major disadvantage of the bithermal fatigue test is that the hold period at zero load during temperature changes may allow the dislocation structure to relax, changing the cyclic stress-strain response ${ }^{88}$.

\subsubsection{Thermal Strain Compensation}

Thermal expansion and contraction of the test specimen (thermal strain) when subjected to thermal cycling has a first-order effect in strain-controlled TMF tests. To obtain a fixed 
mechanical strain range, an accurate means of thermal strain compensation is necessary. The desired mechanical strain command can be obtained from the following equation:

$$
\varepsilon_{\mathrm{rot}}=\varepsilon_{\mathrm{th}}+\varepsilon_{\mathrm{mech}}
$$

where $\varepsilon_{\text {tot }}$ is the total strain command, $\varepsilon_{\mathrm{th}}$ is the compensating thermal strain command, and $\varepsilon_{\text {mech }}$ is the desired mechanical strain. It is necessary to emphasize that, total strain in this case refers to the sum of the compensating thermal strain and mechanical strain as opposed to the standard LCF definition of total strain, i.e. total strain is the sum of inelastic and elastic strain components. Mechanical strain in this case refers to the sum of the inelastic and elastic components of strain, or the strain component that controls the deformation of the test specimen. Therefore, total strain is the command signal driving the strain-control system, and effectively the mechanical strain is the only strain component 'seen' by the test specimen. If one uses the compensating thermal strain as the input to the strain-control loop during thermal cycling, then no load will be imposed on the test specimen. Referring to Figure 3-4 and Figure 3-5, the relationship between the mechanical, thermal and total strain components for OP and IP-TMF tests are clearly illustrated.

Two methods of thermal strain compensation have been used for TMF testing. Both methods are based on determining the free thermal expansion and contraction during thermal cycling, while the test system is in zero force control. 
In the first method, thermal strain is actively compensated for based on real-time measurement of the specimen temperature ${ }^{6,89,90,91}$. In this method, a function $\mathrm{f}(\mathrm{T})$ correlates the thermal strains within the gage section, based on a single temperature measurement. The correlation coefficient, is referred to as the effective coefficient of thermal expansion (ECTE), and the thermal strain can be expressed as a linear function of temperature:

$$
\varepsilon_{t h}=\alpha^{*} T+C
$$

where, $\varepsilon_{\text {th }}$ is the calculated thermal strain, $\alpha^{*}$ is the ECTE, T is the real-time temperature measurement, and $\mathrm{C}$ is a constant. This equation relies on a linear thermal strain response with temperature, which is frequently not the case for large temperature ranges. For nonlinear thermal strain response Castelli ${ }^{89}$ and Jones $^{6}$ utilized piece-wise continuous linear functions. Occasionally, functions representative of the heating and cooling portions of the cycle are necessary ${ }^{92}$ because the temperature response in the controlled gage section may vary between the heating and cooling portions of the temperature cycle.

Another approach for thermal strain compensation that has been used by many researchers in the TMF testing field is recording the thermal strain as a function of time $e^{13,34,93,94}$. In this method, the free expansion/contraction thermal strain is recorded as a function of cycle time prior to commencing a TMF test. The compensating thermal strain command is later accessed at the appropriate time increment during the TMF cycle. 
Pioneers in the TMF testing field utilized Data Trak $^{\gamma}$ systems to record the thermal expansion $^{34,95}$, which compromised the accuracy of thermal strain compensation due to the fact that a hand drawn curve is used to represent the thermal strains ${ }^{95}$. Modern systems utilize computers, which digitally record the thermal strains as a function of time inherently giving much higher accuracy than Data Trak systems since the actual thermal strain values can be recalled automatically at the appropriate time during the TMF cycle.

It is important to note that the thermal strain, for both thermal strain compensation methods, must be recorded when a stabilized thermal strain response has been attained. Usually a number of thermal cycles may be necessary for thermal stabilization to occur. The primary reason for recording the stabilized thermal strain response is to prevent control errors from small variations in temperature occurring during the cycle or from one cycle to the next ${ }^{89}$.

The proposed TMF testing standard ${ }^{92}$ specifies that the accuracy of the thermal strain compensation method be checked prior to commencing a TMF test. The accuracy is assessed during thermal cycling in strain control, with the thermal strain compensation routine actively compensating for the thermal expansion and contraction of the test specimen. The maximum load imposed on the specimen during the duration of the TMF test, arising from a deviation of the specimen thermal strain response from the thermal strain compensation function, is limited to 2 percent of the absolute value of the maximum tensile or compressive load expected during the TMF test.

\footnotetext{
${ }^{\gamma}$ Data Trak is a waveform generator that follows a hand drawn profile on a metallized rotating drum.
} 


\subsection{Heating/Cooling Methods}

One means of specimen heating used in TMF testing is direct resistance heating. This heating method has two potentially serious drawbacks. First, since the direction of current flow is along the axis of the test specimen, the presence of a fatigue crack causes a nonuniform temperature distribution due to localized heating at the crack tip ${ }^{96}$. Second, during final fracture of the test specimen, localized melting can occur at the fracture surfaces due to arcing, making post-failure analysis of the fracture surfaces impossible.

Pernot and Mall $^{79}$ have used a quartz lamp heating technique for TMF testing of CT specimens and Koster, et al. ${ }^{34}$ have used a radiant heating furnace consisting of four $1500 \mathrm{~W}$ quartz lamps. The heating and cooling rate for this type of heating arrangement is highly dependent on the emissivity of the product being heated. For example, highly reflective (i.e. low emissivity) materials such as alumina are difficult to heat by this method $^{87}$. This method of heating presents little problem in TMF testing applications with superalloys, owing to the fact that superalloys readily oxidize at temperatures above $600^{\circ} \mathrm{C}^{97}$, which increases the emissivity of the material.

Direct Induction heating is the most popular means of specimen heating in TMF testing ${ }^{87}$. Referring to Figure $3-6^{98}$, which shows a schematic diagram illustrating the basic concepts of induction heating, notice that the induced eddy currents are in the cirumferential direction, thereby minimizing localized heating of the fatigue crack tip. High heating rates are obtained by a high power density, for which a high frequency generator is preferred due to highly localized heating by the 'skin effect'; however, lower 
frequency induction generators produce lower wall thickness gradients than do higher frequency heaters causing less grounding problems than RF units ${ }^{95}$.

Since the intention of this type of test is to simulate the behavior of a small element of material, strict control of transverse and axial temperature gradients is essential during thermal cycling. It has been noted that specimen instability, referred to as barreling, has been observed in TMF testing, an extreme example of which is illustrated in Figure 3-7 $7^{89}$. Barreling is a cyclic ratcheting process that has been attributed to dynamic hot spots ${ }^{89}$, and inelastic strain range ${ }^{99}$. Therefore, it is essential that the axial temperature gradients be carefully controlled during thermal cycling in TMF testing. The proposed TMF testing standard ${ }^{92}$ limits gage section axial and transverse temperature gradients to $\pm 1.5 \%$ of the maximum test temperature or $\pm 3^{\circ} \mathrm{C}$, whichever value is greater. To comply with this specification a compromise between heating rate and obtaining an acceptable temperature distribution is often necessary ${ }^{10}$. The temperature cycling rate in TMF testing is dependent on the specimen geometry, temperature range, and the method for cooling the specimen. Typical heating rates are 1 to $5^{\circ} \mathrm{C} / \mathrm{sec}$ for solid specimens and 10 to $50^{\circ} \mathrm{C} / \mathrm{sec}$ for tubular specimen geometries ${ }^{87}$.

It is also essential to consider the phasing accuracy of the thermal cycle with-respect-to the mechanical strain cycle ${ }^{89}$. Considering that one TMF cycle is of the order of seconds or minutes, the response of the load frame to a change in command is practically instantaneous. Temperature response on the other hand, is generally much slower, especially during temperature command reversals. The proposed TMF testing standard ${ }^{92}$ 
has placed a limit on the phasing accuracy of the temperature and mechanical strain waveforms; the maximum permitted phasing error is $\pm 5^{\circ}$.

In many cases, the primary means of specimen cooling is by conduction, to the cooler specimen grips. Forced-air cooling has also been used in TMF testing to permit higher temperature cycling rates and lower temperature ranges ${ }^{93,94}$. This approach to specimen cooling is discouraged, since it can result in higher transverse and axial temperature gradients. Zamrik et al. ${ }^{94}$ observed a higher number of cracks initiated at the inner surface of an internally forced-air cooled specimen than the same tests without forced-air cooling. This was attributed to thermal fatigue resulting from a through thickness temperature gradient introduced by the cooling air.

As described previously, thermal strain has a first-order effect on the control variable. In fact, the thermal strains at elevated temperatures can exceed the mechanical deformation, and therefore it is essential that the temperature be measured and controlled with a great deal of certainty and repeatability, especially during temperature reversals. Two frequently utilized methods for temperature feedback and control in TMF testing include thermocouples and optical pyrometry. In many cases when thermocouples are used for temperature measurement, they are mechanically attached to the specimen. Direct attachment of thermocouples by spotwelding is discouraged, especially for coated materials, which are easily damaged during the spotwelding process ${ }^{87}$. Optical pyrometry is particularly useful for temperature control because it is non-contacting; however, 
preoxidizing to obtain a stable oxide layer, and careful calibration of the emissivity are necessary for accurate temperature measurement.

\subsection{Specimen Design For TMF Testing}

Many different types of specimens have been used in TMF testing. They are (1) solid LCF-type specimens ${ }^{89,100}$, (2) tubular LCF-type specimens ${ }^{34,80.89}$, (3) single edge notch specimens $^{93}$, (4) solid rectangular section specimens ${ }^{81}$, and (5) compact tension specimens ${ }^{79}$. Most TMF testing is conducted with smooth fatigue specimens that are either solid or tubular. For nickel-base superalloys, which possess a relatively low thermal conductivity, tubular type specimens are preferred for reducing transverse temperature gradients ${ }^{10}$. With tubular specimens, the wall thickness must be sufficient to prevent specimen instability; a wall thickness of $1-1.5 \mathrm{~mm}$ is typically utilized ${ }^{10,89,94}$. To preserve isotropy in polycrystalline materials, $10-20$ grains through the wall thickness of a tubular specimen is specified in the proposed TMF testing standard ${ }^{92}$. Most tubular TMF specimens feature an extended gage section to minimize axial temperature gradients. A typical example of a tubular specimen is illustrated in Figure $3-8^{10}$.

\subsection{Data Acquisition}

Data acquisition systems in pioneering work generally consisted of chart recorders, for continuous monitoring of temperature, load and total strain as a function of time, and X$Y$ plotters for acquiring stress-strain hysteresis loops ${ }^{80,95}$. Presently, digital data acquisition and control systems are utilized in state-of-the-art TMF testing 
facilities ${ }^{6,78,81,89,93}$. Typically, temperature, total strain, and load readings are digitally recorded as a function of cycle time for later analysis. The proposed TMF testing standard $^{92}$, as well as ASTM E $606^{82}$, recommend recording the initial series of hysteresis loops (i.e., cycles 1 and 2), and subsequent hysteresis loops at logarithmic intervals of fatigue life. ASTM E $606^{82}$ makes one further stipulation that a minimum of 10 additional hysteresis loops should be recorded for tests of greater than 100 cycles in duration. It is also desirable to monitor and record the cyclic maximum and minimum values for load and total strain continuously during testing to capture the cyclic response of the test specimen.

\subsection{TMF Data Analysis}

\subsubsection{Stress-Strain Hysteresis Loop Components}

Complications can arise in the analysis of TMF hysteresis loops. The first complication arises from the fact that total strain is the controlled variable in TMF testing, and it is total strain-load data pairs that are recorded to define the hysteresis loop. Therefore, a means of reducing the total strain to the mechanical strain component is necessary, and given as:

$$
\varepsilon_{\text {mech }}=\varepsilon_{\text {sot }}-\varepsilon_{\text {th }}
$$

where $\varepsilon_{\text {mech }}$ is the mechanical strain, $\varepsilon_{\text {tot }}$ is the total strain feedback from the strain transducer, and $\varepsilon_{\mathrm{th}}$ is the commanded thermal strain increment. The other problem arises when determining the inelastic and elastic strain components from the stress-strain 
hysteresis loop. Several potential options for determining the inelastic strain include: (1) the width of the hysteresis loop at mean stress (BS7270:1990 ${ }^{101}$ ), (2) the width of the hysteresis loop at the abscissa ${ }^{100}$, and (3) the relation $\Delta \varepsilon_{\text {lot }}-\Delta \sigma / E$ (ASTM E606-92 ${ }^{82}$ ) where $\Delta \varepsilon_{\text {tot }}$ is the total strain (mechanical strain) and $\Delta \sigma$ is the stress range. It is generally accepted that the definition of inelastic strain range in a TMF hysteresis loop is the width of the hysteresis loop at the abscissa (i.e., zero stress). Figure 3-9 illustrates a schematic view of a TMF stress-mechanical strain hysteresis loop defining the stress and strain parameters that are typically used for TMF or IT-LCF data analysis ${ }^{100}$.

\subsubsection{Definition of Failure Life}

During TMF or LCF testing, the complete separation of ductile materials is approached by a gradual change in load with each cycle. Since the intention of TMF tests is to provide information that can be used to predict the fatigue life for critical locations in components, it is usually necessary to have knowledge of the life to crack initiation. The definition of crack initiation or failure, in TMF or IT-LCF, is dependent on many factors including, test control mode, specimen geometry, and crack detection method ${ }^{102}$. Several methods determining the fatigue crack initiation life include: (1) fixed percentage load drop from the stabilized cyclic tensile load, (2) specific surface crack size determined by surface replication techniques ${ }^{13}$, and (3) specific potential drop when utilizing potential drop techniques ${ }^{93}$.

In strain-controlled tests, the presence of a macroscopic fatigue crack can be interpreted from changes in the hysteresis loop and cyclic stress response. For instance, when a 
macroscopic fatigue crack is present in a ductile material, the load will continuously decrease to maintain the same strain limits. The observed change in cyclic tensile load response can be difficult to apply in practice for materials that do not exhibit a stabilized tensile cycle stress response. For materials that do not cyclic stabilize, the presence of a macro-crack can be detected by plotting the maximum cyclic tensile stress versus number of cycles; a sudden increase in the slope indicates the presence of a macro-crack.

While changes to the cyclic tensile stress response, when a macro-crack is present are easily detected, changes to the cyclic compressive load carrying capability of the specimen are subtle and only appear as changes to the compressive portion of the hysteresis loop. During the tensile loading portion of the fatigue cycle, a small portion of material is ruptured, resulting in extrusions on the fatigue crack faces. With increasing compressive load these extrusions collapse, and the full compressive load carrying capability of the material is restored ${ }^{102}$. This behavior is clearly demonstrated by the inflection point seen in compressive portion of the hysteresis loops, when a substantial fatigue crack is present (see Figure 3-10). The onset of inflection in the hysteresis loop can also be used to detect crack initiation, but this method has been found to be insensitive ${ }^{103}$.

The most frequently used method for determining the life to crack initiation in cylindrical fatigue specimens is a fixed percentage load drop from the cyclically stabilized tensile load. Many different values for percentage cyclic load drop can be used to define crack initiation life such as $20 \%{ }^{104}$. It has been noted for some materials, especially when 
conducting OP-TMF tests, that only a very small load drop will occur before complete separation of the test specimen. Bonacuse and $\mathrm{Kallur}^{105}$, found that a $10 \%$ cyclic load drop was unacceptable for axial-torsional TMF tests on specimens made from Haynes 188 because catastrophic failure occurred before attaining the $10 \%$ load drop. They resorted to a $5 \%$ load drop to define life to crack initiation, which proved to be satisfactory.

\subsection{Summary}

From the preceding discussion one can identify several requirements for TMF testing. First strain-control is necessary to simulate the thermal loading conditions imposed on a component at high temperature. In this case, axial strain-control is preferred to diametral strain-control due to control complications prevalent under transient thermal conditions. Second, controlled heating and cooling are necessary to simulate a strain-temperature relationship that is seen by a component in service. It is essential that the temperature distribution in the uniaxial specimen be optimized to obtain volume element representation and that temperature measurement and control is accurate and repeatable to maintain accurate thermal strain compensation. The last condition is that one needs to have data acquisition capability to record deformation data (hysteresis loops), and the cyclic behavior of the component. The data acquisition component is essential for recording the thermal expansion and contraction of the material for thermal strain compensation, and for maintaining synchronization between the strain and temperaturecontrol loops. 


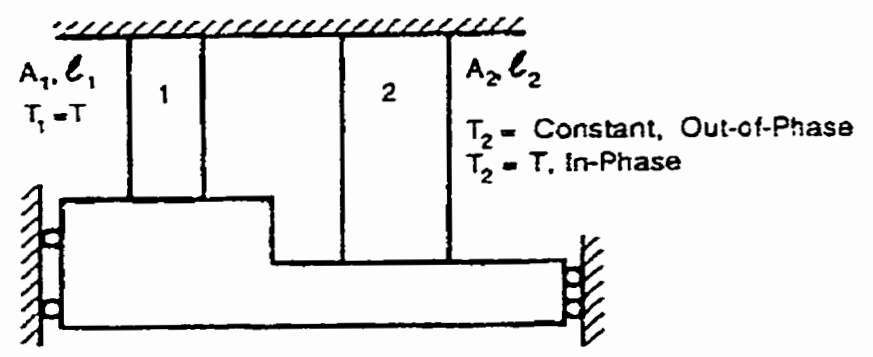

(a)

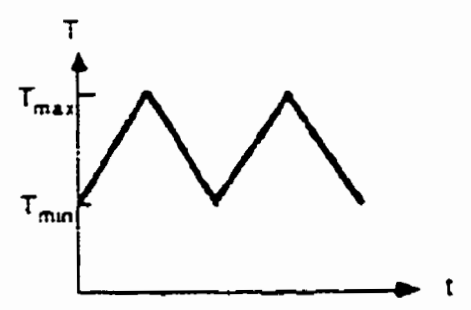

Figure 3-1 - Schematic view of a two-bar structure for TMF testing ${ }^{77}$.

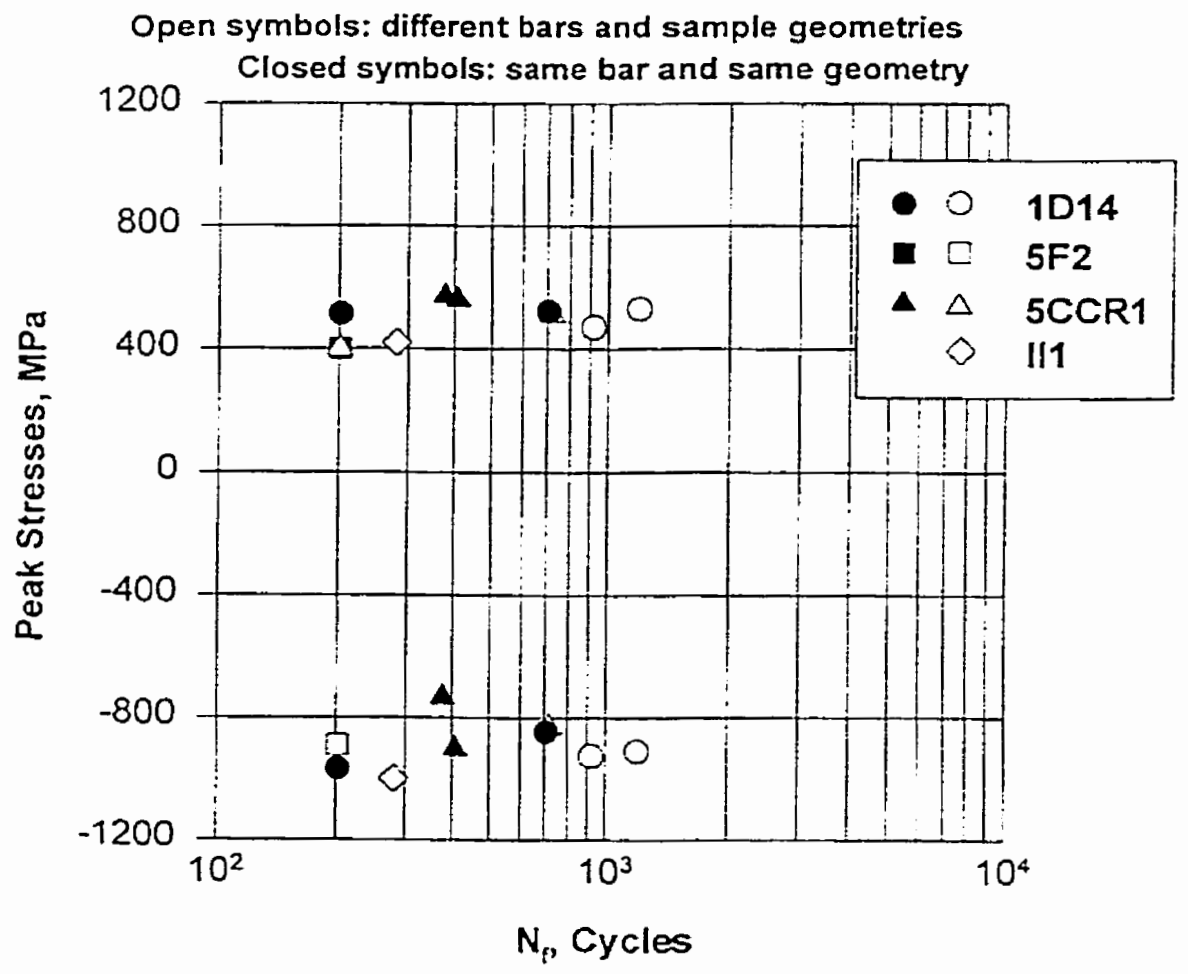

Figure 3-2 - S-N results for the TMF interlaboratory test program conducted under Cost 501 Round 2 using the same temperature and mechanical strain cycles ${ }^{81}$. Note: each code in the legend refers to the different participant's project designation. 


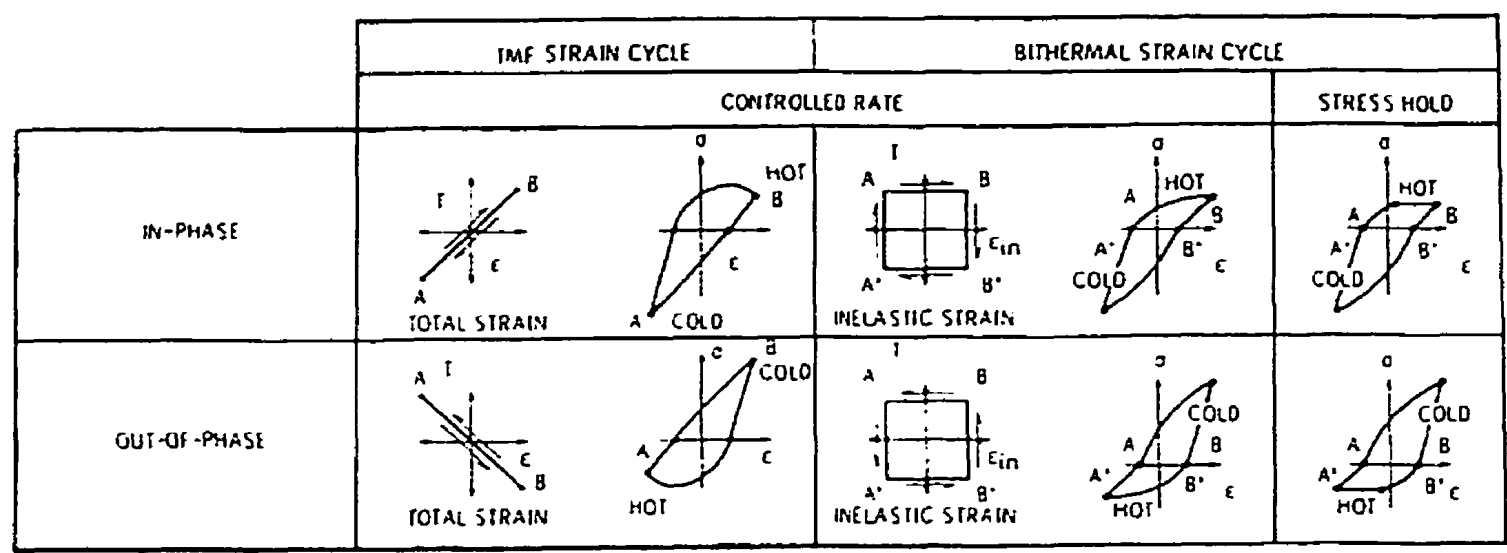

Figure 3-3 - Comparison between in-phase and out-of-phase TMF and bithermal fatigue cycles $^{88}$.

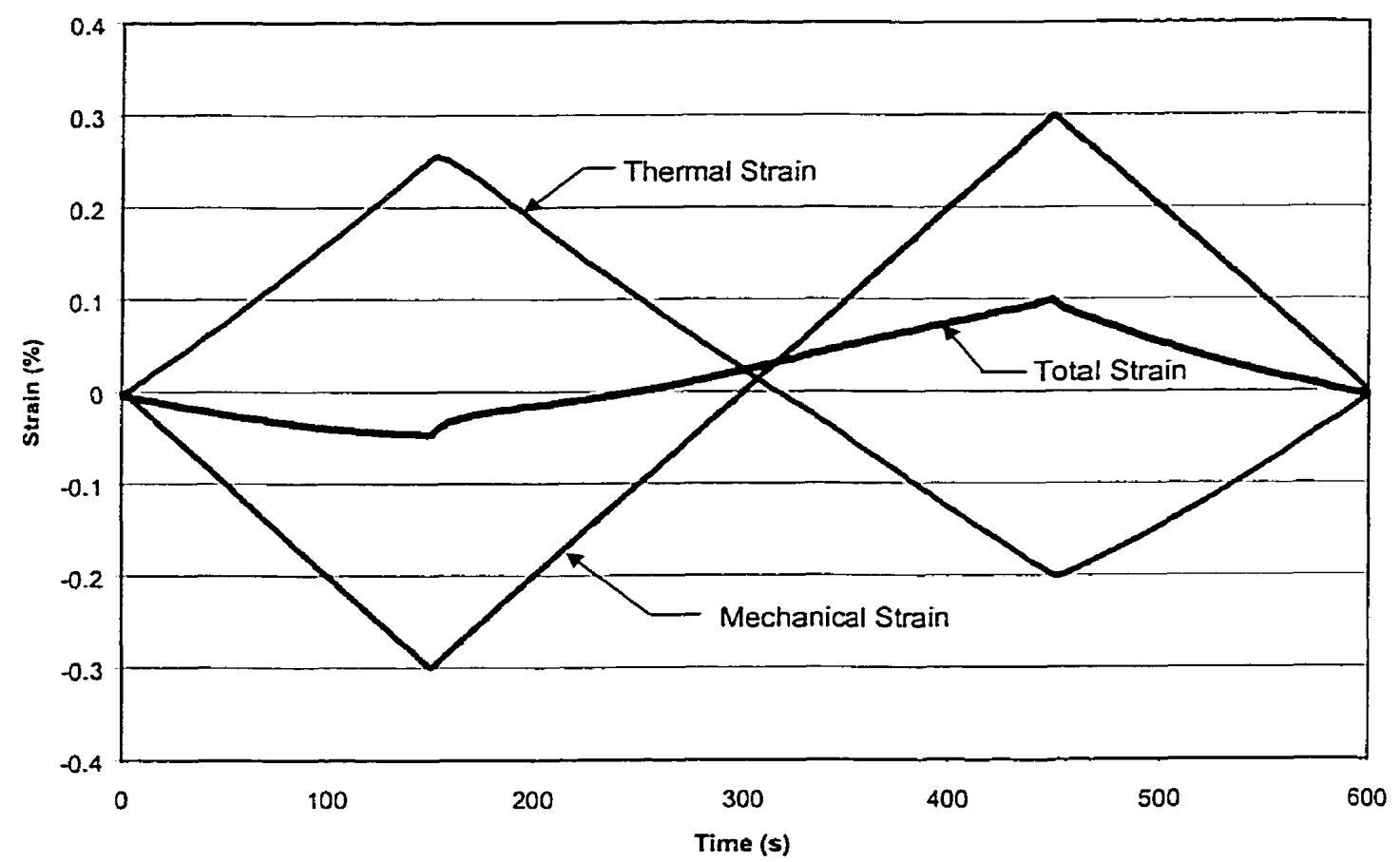

Figure 3-4 - Relationship between thermal, mechanical and total strain components for an out-of-phase TMF test. 


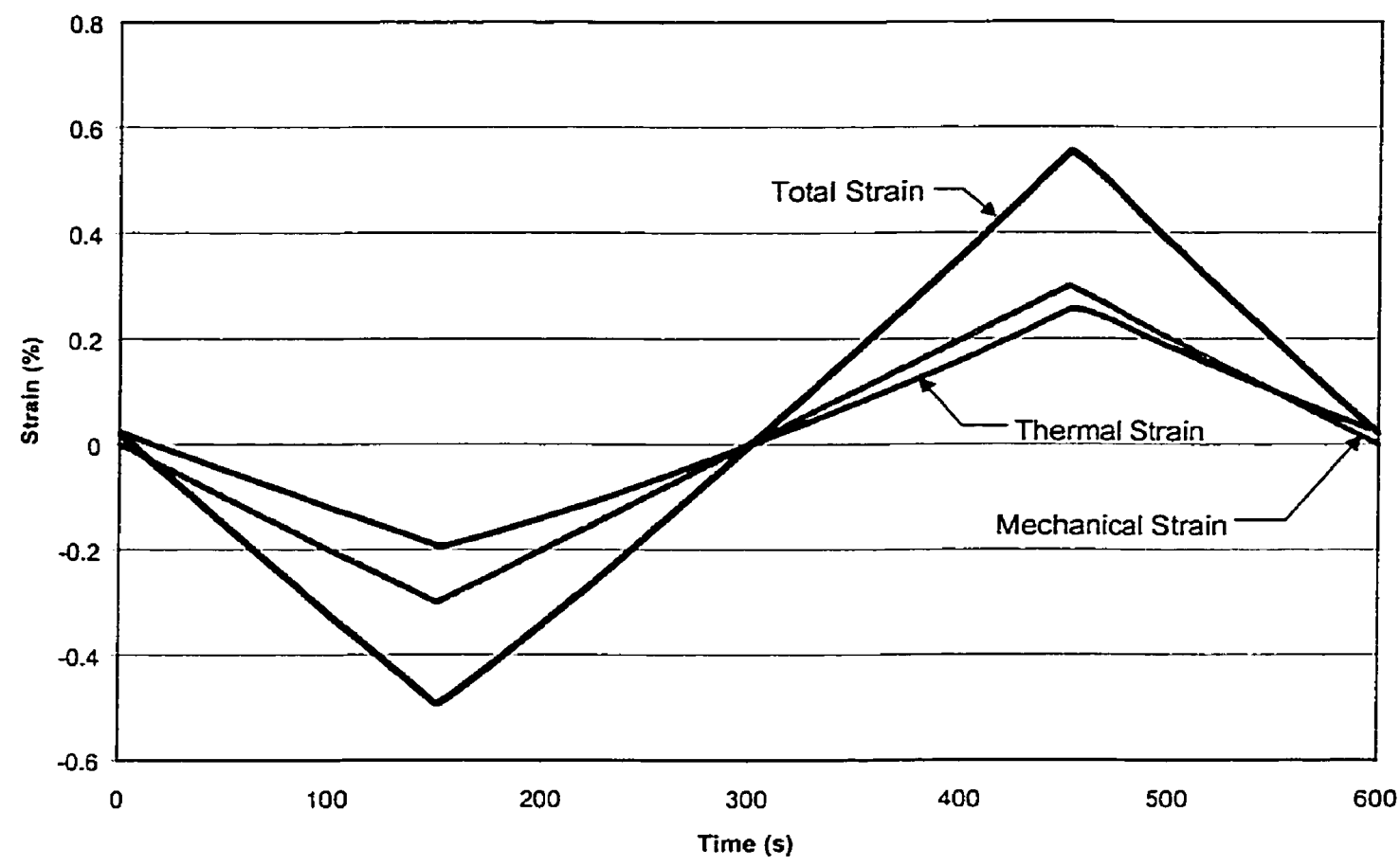

Figure 3-5 - Relationship between thermal, mechanical and total strain components for in-phase TMF tests.

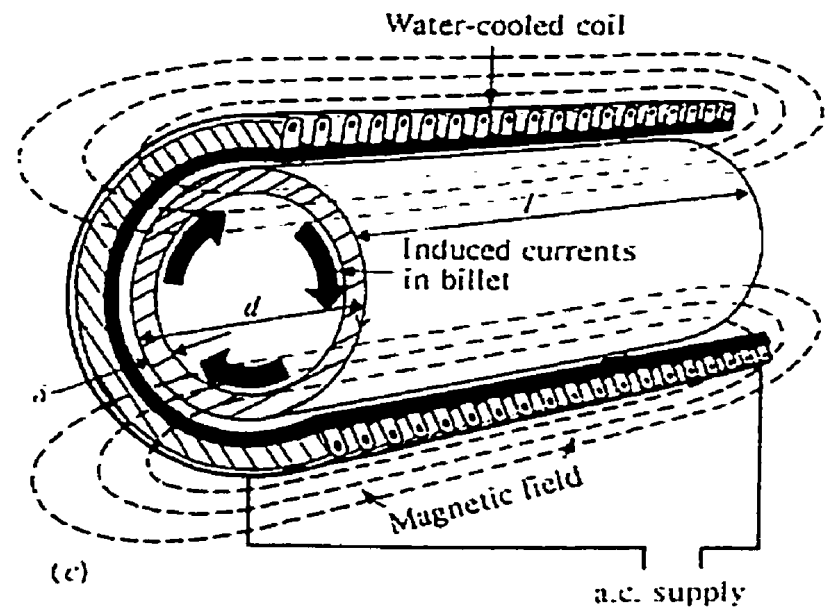

Figure 3-6 - Basic principles of direct induction heating ${ }^{98}$. 


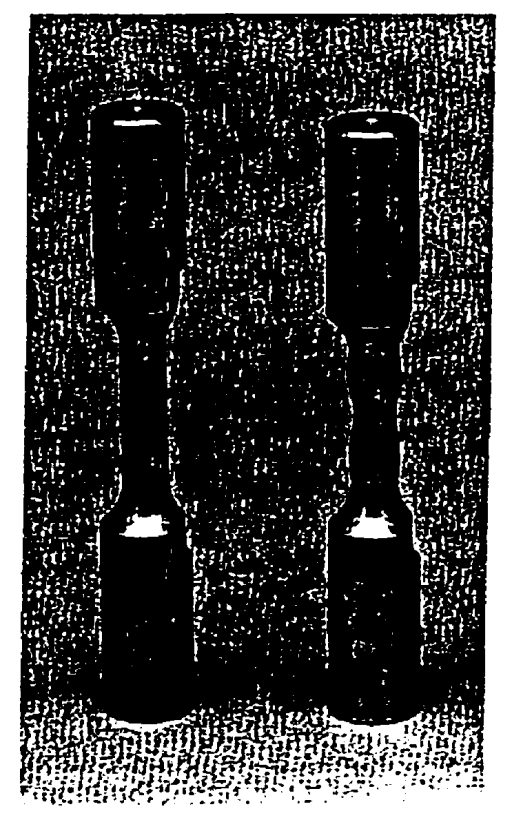

Figure 3-7 - Specimen before and after cyclic barreling induced by $\mathrm{TMF}^{89}$.

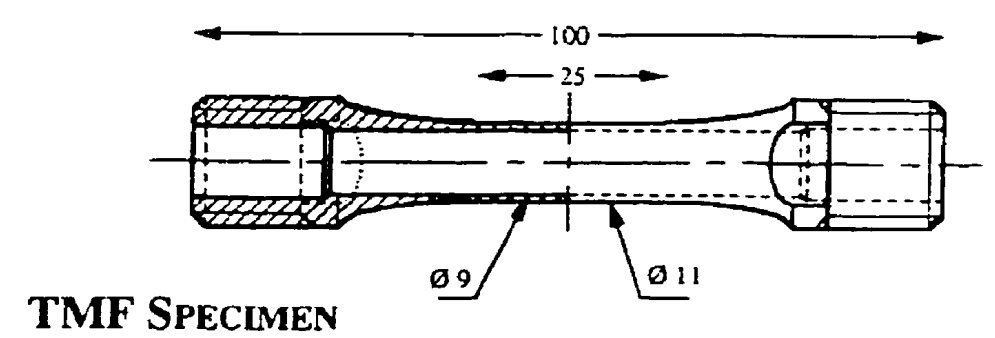

Figure 3-8 - Tubular TMF specimen used by Remy ${ }^{10}$; others employ similar tubular specimen geometries for TMF testing. Notice the extended gage section used to facilitate obtaining a uniform axial temperature gradient. 


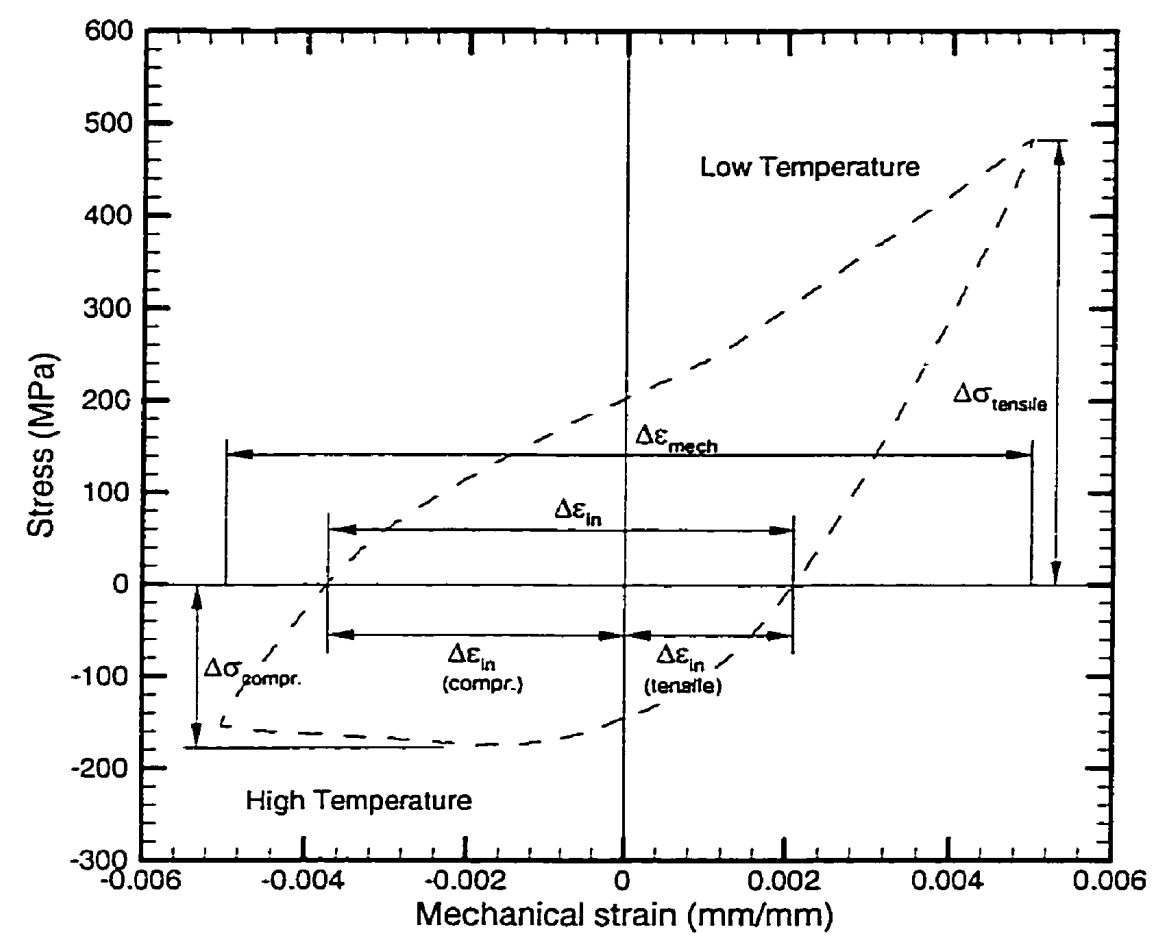

Figure 3-9 - A schematic view of a TMF hysteresis loop defining the stress and strain parameters.

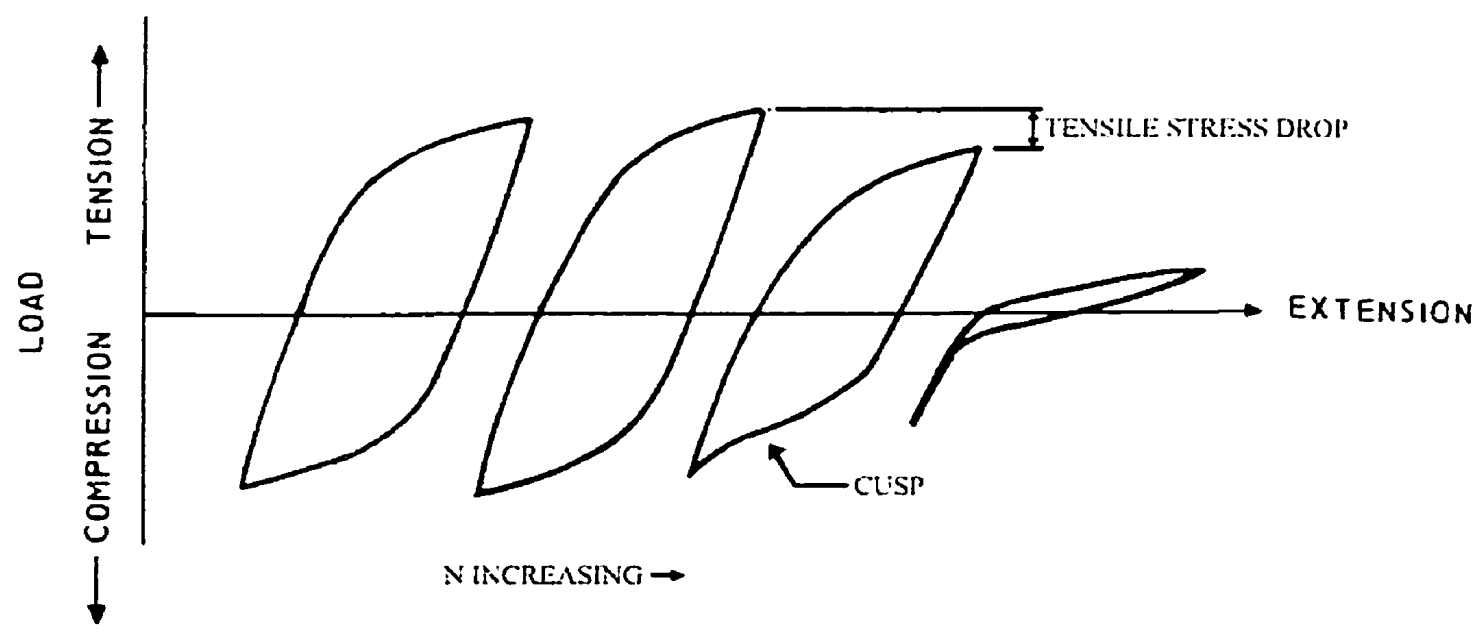

Figure 3-10 - Hysteresis loops formed during LCF testing illustrating stress amplitude (tensile) drop and cusp formation associated with the presence of a macrocrack. 


\section{TMF Testing Facility and Experimental Approach}

\subsection{Introduction}

From the previous Chapter, the general requirements for strain controlled thermalmechanical fatigue (TMF) testing can be summarized as follows:

1. Strain control to simulate thermal loading conditions imposed on a component at high temperature,

2. Controlled heating and cooling to simulate a strain-temperature relationship that may be seen by a component in service and,

3. Data acquisition capability to record deformation data (hysteresis loops) and the cyclic behavior of the component.

As part of this thesis research, a testing facility has been designed to meet these three requirements. The experimental system is capable of conducting strain-controlled uniaxial TMF, and IT-LCF tests. The strain-temperature relationships can be varied independently through two closed loops, one for temperature control, and the other for axial strain control. Presently, the testing software developed for the TMF facility is capable of in-phase and out-of-phase strain-temperature relationships with triangular command waveforms; however, the software can be easily changed to suit other TMF testing requirements. The TMF testing facility and the TMF testing software are 
described in the following sections. The choice of experimental method in the present work is based on pre-existing equipment, and the guidelines summarized in the previous chapter.

\subsection{General Overview of the NRC-IAR TMF Testing Facility}

The TMF facility developed as part of this thesis at the Institute for Aerospace ResearchNRC is illustrated in Figure 4-1. The facility consists of two independently programmable control-loops, one for temperature control, and the other for mechanical loading of the test specimen. Figure 4-2 is a general block diagram of the TMF testing facility illustrating the temperature and strain control loops. A multifunction data acquisition board, programmed through a $\mathrm{LabVIEW}^{\gamma}$ program specifically created for TMF and IT-LCF testing, generates the command signals to each respective control loop. The following sections discuss the hardware configurations for IT-LCF and TMF testing, modifications to some of the equipment and the software that was created to control the IT-LCF and TMF tests.

\subsection{Mechanical Loading System}

The mechanical loading system consists of an MTS model 810 servo-controlled, electrohydraulic material testing system, which includes a load frame, hydraulic actuator, electrohydraulic servo valve system, load cell, axial extensometer, actuator position LVDT, and a MTS 407 analog load-unit controller for closed-loop control (see Figure

\footnotetext{
${ }^{\gamma}$ National Instruments, Austin Texas 78759-3504
} 
4-1). The load-unit controller has analog I/O signal connections and a built-in digital interface for remote programming via serial I/O (RS-232). With a serial I/O connection, and the supplied LabVIEW drivers, it is possible to control and program the load-unit controller from a host computer. Loads can be imposed on the specimen by force, actuator displacement or strain control. The load-unit controller provides an analog input for command signal programming from an external source, such as a function generator. Continuous acquisition of transducer feedback signals is facilitated by external analog signal connections on the rear panel of the load-unit controller.

Water-cooled hydraulic wedge grips (MTS Model 647.10) are used to secure the ends of the test specimen. The clamping force provided by the grips is adjustable to prevent backlash during fully reversed cyclic loading. A 4340 steel trial specimen equipped with 12 strain gages was used to check the specimen-load train alignment prior to testing. The trial specimen and strain gage configuration is shown schematically in Figure 4-3. A dedicated data acquisition system and software (MTS EZ-Alignment Model 709.20A-01) was used to monitor the real-time strain readings from each of the strain gages. To facilitate specimen-load train alignment, an alignment fixture (MTS Model 609.10A-01) was installed between the load cell and test machine crosshead. The concentric and angular alignment settings were adjusted to minimize bending strains, following the procedures given in the alignment fixture instruction manual (MTS Part No 15031901A). After the final alignment adjustments were completed, strain readings were taken for a range of applied loads about a reference plane. Then the specimen was rotated $90^{\circ}$ clockwise and the strain readings were recorded for a range of applied loads. This process 
was repeated until the specimen was retumed to its original position. Table 4-1 summarizes the average of three bending strain readings measured for an axial load of $5 \mathrm{kN}$.

The bending strain requirements for LCF testing are given in ASTM E606 ${ }^{82}$. This standard limits the maximum bending strain to $\pm 5 \%$ of the minimum strain amplitude in the test program. Referring to Table 4-I, it is clearly seen for two specimen positions that the bending strain readings exceeded the maximum bending strain specified in ASTM E606 $6^{82}$. It should be noted that when the specimen ends are clamped in the specimen grips, that the ends of the specimen are deformed by the raised serration's on the grip wedge faces. When viewing the specimen from the grip end, the serration pattern is not symmetrical in a circumferential sense, and consequently it is impossible to grip the specimen in the same serration indentations when gripping the specimen in the $90^{\circ}$ and $270^{\circ}$ positions. It is suspected that these two anomalous bending strain readings may be partially due to this situation since they coincide with the $90^{\circ}$ and $270^{\circ}$ positions. During the commissioning tests conducted in this work, there was no indication that fatigue cracks initiated in preferred circumferential positions.

\subsubsection{Modifications to MTS 407 Load Unit Controller}

Referring to the control block diagram in Figure 4-2, it can be clearly seen that many pieces of equipment must interact as a unit when conducting TMF and IT-LCF tests. For safe operation, it is necessary to interconnect the hardware between the temperature and strain control loops such that any piece of hardware can stop a test if an equipment limit is exceeded. To facilitate the interconnection of the hardware in each control loop, an 
auxiliary interlock chain was added to the external emergency stop connection at the rear the MTS 407 load-unit controller. A bypass switch has also been added to the auxiliary interlock chain to disable it when not required, e.g. when loading and unloading test specimens. The block diagram for the external interlock chain is illustrated in Figure 4-4.

The second modification to the controller included the addition of an interlock-sense connection between the load-unit controller and the data acquisition computer. The purpose of the interlock-sense connection is to stop the TMF testing software when an interlock is tripped on the load-unit controller. This connection is routed to one of the digital outputs of the 407 controller, which has been configured to switch from high-tolow when an interlock is tripped. The external 5 VDC supply from the data acquisition board energizes the interlock-sense connection, which is monitored by the TMF testing software through one of the analog input channels of the data acquisition board. The block diagram for the interlock-sense connection is illustrated in Figure 4-5.

\subsection{Extensometry}

As stated previously, strain control is one of the requirements for TMF and IT-LCF testing. Axial strain control is used in this investigation because of the control problems associated with diametral strain control, which were described in the previous chapter. The two high temperature axial extensometers used for IT-LCF and TMF tests in this investigation are described in the following sub-sections. 


\subsubsection{IT-LCF Tests}

For the IT-LCF tests conducted in the investigation, a water-cooled axial extensometer (MTS Model $632.56 \mathrm{C}-01$ ) with a maximum temperature capability of $1000^{\circ} \mathrm{C}$ was used to control axial strain over the $25 \mathrm{~mm}$ gage length. The extensometer was attached to the specimen by means of two spring loaded attachment rods made of quartz, which apply a lateral force to keep the quartz vee-chisel extension rods in contact with the specimen (see Figure 4-6). The $25 \mathrm{~mm}$ axial gage length extensometer was selected for the IT-LCF tests for two reasons. First, a relatively uniform axial temperature gradient within the specimen gage section was easily achieved under the static temperature conditions, and second because some initial problems with extensometer slip were encountered when using the $12 \mathrm{~mm}$ gage length extensometer, which is described in the following section. It was observed that an oxidized specimen surface together with the correct hold down springs, provided enough friction to prevent the extensometer from slipping during testing.

\subsubsection{TMF Tests}

For the TMF tests conducted in this investigation, an air-cooled axial extensometer (MTS Model $632.41 \mathrm{C}-11$ ) with a maximum temperature capability of $1000^{\circ} \mathrm{C}$ was used to control axial strain over a $12 \mathrm{~mm}$ gage length. An axial gage length of $12 \mathrm{~mm}$ was selected over the $25 \mathrm{~mm}$ gage length used for IT-LCF tests because it is easier to control axial temperature gradients over the shorter test section. The extensometer was located on the specimen gage section by means of a hold-down spring system contained in the heat shield housing. The hold-down spring system provides a lateral force to hold the vee- 
chisel quartz extension rods in place on the specimen (see Figure 4-7). The extensometer housing is specifically designed to attach to a split 'clam-shell' style radiant heating furnace. To correctly locate the extensometer for installation on the test specimen, it was necessary to mount the extensometer housing to a bracket attached to the load frame. A bracket was fabricated in-house for this purpose, but was found to be inadequate because the bracket did not provide the adjustment range necessary to correctly locate the extensometer. To solve this problem, the correct mounting bracket for this extensometer was purchased from MTS. It was observed that an oxidized surface together with proper alignment of the extensometer housing, was effective at preventing the extensometer from slipping during a test.

\subsubsection{Thermal Strain Compensation}

In order to cycle between fixed mechanical strain limits in a TMF test, it is necessary to compensate for the thermal expansion and contraction of the test specimen. In this work, the stabilized thermal strain response of the test specimen was digitally recorded by the data acquisition computer, as a function of cycle time, prior to commencing a TMF test.

Ideally, when the testing machine is in total axial strain control, the thermal strain compensation routine will fully compensate for the thermal expansion and contraction during thermal cycling, i.e., no loads will be imposed on the test specimen. Generally this is not the case because of small variations in the thermal response of the test specimen during thermal cycling. The accuracy of the thermal strain compensation method can be assessed from a plot of stress versus thermal strain. Figure 4-8 shows a plot stress versus 
thermal strain, which represents the typical accuracy of the thermal strain compensation method from this work. During this investigation, the stress imposed on the specimen when using recorded thermal strain data for thermal strain compensation, did not exceed 5.5 MPa.

\subsection{Specimen Heating/Cooling}

Several heating methods were considered for the present study including, radiant heating furnaces, direct radio frequency induction heating, and direct electrical resistance heating. The advantages and disadvantages for each of these heating methods were discussed in the previous chapter. For the present work, Radio Frequency (RF) induction heating was selected since the equipment was available at the LAR-NRC testing laboratory. Forced-air cooling, even if diffused, can cause localized cooling of the specimen leading to unacceptable axial and circumferential temperature gradients, and specimen barreling. Therefore, the cooling phase of a TMF cycle in the present study was unassisted. For higher cooling rates internal or external forced-air cooling systems may be necessary for future testing requirements.

\subsubsection{Induction Heating}

The high frequency generator used in this work (Lepel Model LSS 2.5) has a maximum power output of $2.5 \mathrm{~kW}$ at operating frequencies in the range of 20 to $200 \mathrm{kHz}$. During preliminary testing with this high frequency generator, the maximum heating rate that could be attained between $600-950^{\circ} \mathrm{C}$ with solid IN738LC test specimens was $1.25^{\circ} \mathrm{C}$ per second. Furthermore, to attain the maximum temperature $\left(950^{\circ} \mathrm{C}\right)$ with this heating rate, 
the full power output of the induction heater was required. If a heating rate higher than $1.25^{\circ} \mathrm{C}$ per second is required with solid specimens, as used in this work, a more powerful $\mathrm{RF}$ generator would be necessary. Ellison et al. ${ }^{86}$ recommend a $4 \mathrm{~kW} \mathrm{RF}$ generator for testing temperatures up to $1000^{\circ} \mathrm{C}$ using typical fatigue specimens having gage diameters of 5 to $8 \mathrm{~mm}$. A maximum heating rate of $1.0^{\circ} \mathrm{C}$ per second was used in the present work to minimize the deviation between the temperature command and feedback during temperature cycling.

\subsubsection{Heating Coils}

One of the most important parameters in induction heating is the geometry of the heating coil. The geometry of the heating coil controls both the efficiency of energy transfer to the work piece, and the temperature uniformity ${ }^{98}$. In multi-turn coil designs, two parameters can be varied to change the temperature distribution in the work piece, 1) the axial pitch of the coils (the distance between coil turns), and 2) the coupling (the distance between a coil turn and the work piece). $\mathrm{Au}^{102}$ gives some useful guidelines to follow when designing a heating coil. Good temperature uniformity can generally be achieved by using extended grip sections or extended gage length specimens in which the extended sections primarily serve to couple the RF heating coil. By this method, heat is conducted from the extended sections to the gage section of the test specimen. Starting with the guidelines in Reference 102, an acceptable heating coil was evolved by a trial and error process. 
In the present study, two induction heating coils were used. Two heating coils were necessary since two different specimen geometries were used for the IT-LCF and TMF tests (see Chapter 5). Furthermore, it was observed in this work that a suitable coil design for obtaining a uniform static temperature distribution was inadequate for obtaining a uniform dynamic temperature distribution during thermal cycling. Both heating coils used in this study were fabricated from copper tubing wound around different size mandrels to give the coupling required for obtaining an acceptable temperature distribution. The coil windings were spaced to accommodate the axial extensometry and provide access for temperature measurement. To prevent short-circuits, the heating coils were covered with Nextel $312^{*}$ ceramic fiber insulation.

\subsubsection{Heating Coil for IT-LCF Testing}

The IT-LCF heating coil was fabricated from 3/16-inch $(4.8 \mathrm{~mm})$ copper tubing, and consisted of six turns with three distinct heating zones providing different couplings. To facilitate access for the $25 \mathrm{~mm}$ gage length extensometer and achieve an acceptable axial temperature distribution, the heating coil arrangement consisted of two 0.90 -inch (22.9 $\mathrm{mm}$ ) ID turns located at each end of the extended gage section, and two 1.10-inch (27.9 mm) ID turns at the center of the specimen gage section (see Figure 4-6). The static temperature distribution was adjusted by altering the pitch between the individual coil segments. Using this heating coil arrangement, the axial temperature gradient did not exceed $2 \%$ for $85 \%$ of the gage length, and $5 \%$ between the points of extensometer attachment.

\footnotetext{
- Nextel is registered trademark of the 3M Company
} 


\subsubsection{Heating Coil for TMF Testing}

It was determined through the process of trial and error that the best coil configuration for dynamic temperature cycling is a coil with several major turns supplying the majority of heat input at the ends of the uniform gage section. The heating coil for TMF testing was fabricated from 1/8" (3.2 mm) copper tubing, and consisted of three major turns of $0.95-$ inch $(24.1 \mathrm{~mm})$ ID to heat the ends of the uniform gage section, and one central turn of 1.20-inch (30.5 mm) ID (see Figure 4-7). This heating coil arrangement provided sufficient access to the specimen for temperature measurement, and for installation of the $12 \mathrm{~mm}$ gage length axial extensometer. During temperature cycling trials at a temperature rate of $1^{\circ} \mathrm{C}$ per second between $750-950^{\circ} \mathrm{C}$, the maximum axial temperature gradient at the hot and cold end of the temperature cycle was $4.5^{\circ} \mathrm{C}$, and $11^{\circ} \mathrm{C}$, respectively (see Figure 4-9). Circumferential temperature gradients did not exceed $\pm 2^{\circ} \mathrm{C}$ during dynamic temperature cycling trials.

\subsubsection{Optimization of Gage Section Temperature Distribution}

The existing ASTM IT-LCF ${ }^{82}$ and proposed ASTM TMF ${ }^{92}$ testing standards specifically state the importance of controlling the axial, transverse and circumferential temperature gradients within the test section. Obtaining an acceptable temperature distribution when using induction heating is challenging since the heat input tends to be highly localized which results in hot spots ${ }^{89}$. The axial and circumferential temperature gradients for both induction coils used in this work were measured using 0.010 -inch $(0.254 \mathrm{~mm})$ diameter type-K thermocouples spot-welded to the surface of a IN738LCF calibration specimen. One of the drawbacks of using thermocouples for temperature measurement is that the 
temperature measurement is localized to its point of attachment. Therefore, to detect the presence of a hot spot, the thermocouples must be spaced relatively close to one another. Castelli et al. ${ }^{89}$ suggest an axial spacing of $6 \mathrm{~mm}$ to keep the thermocouples sufficiently close together to determine the location of hot spots. The thermocouple configuration used to measure axial temperature gradients is shown in Figure 4-10. The thermocouple leads were oriented radially through the RF field, as suggested by Sumner ${ }^{106}$, to minimize self-inductance. The circumferential temperature distribution was determined by rotating the calibration specimen $180^{\circ}$ from its initial position.

The first step to optimizing the temperature distribution in the calibration specimen was to preoxidize the specimen so that a stable oxide layer was attained for subsequent temperature measurement and control with the infrared pyrometer. Following this step, both heating coils were adjusted to obtain an acceptable static temperature profile at the maximum test temperature used in this work $\left(950^{\circ} \mathrm{C}\right)$. Generally, it was only necessary to change the pitch of the individual coil windings to obtain an acceptable static temperature profile. After an acceptable static temperature was attained, no further work was necessary for the IT-LCF heating coil. It was observed during thermal cycling that a small static temperature gradient $\pm 2^{\circ} \mathrm{C}$ increased to a much larger gradient (approximately $\pm 10^{\circ} \mathrm{C}$ ) during thermal cycling. Therefore, it was necessary to optimize the TMF heating coil to minimize axial temperature gradients under dynamic temperature cycling. To minimize the dynamic axial temperature gradients, it was necessary to change both the pitch and coupling of the TMF heating coil through a trial-and-error process. 


\subsubsection{Modifications to the RF Induction Heater}

Several modifications to the RF generator were required to ensure the safety of the operator and the control system hardware. First, the RF generator was wired internally such that energizing the heating coil required a run-relay to be closed. The run-relay served the purpose of preventing one from inadvertently energizing the induction coil when a test had finished, and to de-energize the heating coil when the TMF test software was stopped by a program error or user intervention. The second modification was to install an interlock connection between the RF generator and the MTS 407 controller. The purpose of this connection was to trip a load-unit controller interlock when the heatoff switch was pressed or when a RF generator fault occurred during a test. A bypass switch was added to the interlock and run-relay connections to permit manual operation of the RF generator. A schematic view of the induction heater modifications is presented in Figure 4-11.

\subsection{Temperature Measurement and Control}

Two temperature measurement methods were considered in the present study: 1) fine wire thermocouples spot-welded to the specimen surface and 2) infrared pyrometry. The advantages and disadvantages of both temperature measurement and control methods were discussed in the previous chapter. For the present work, an infrared pyrometer was selected as the means of temperature measurement and control because of its inherent simplicity, and equipment availability in the IAR-NRC testing laboratory. 


\subsubsection{Temperature Control Loop}

The TMF testing facility employs a closed-loop temperature control system that connects together the RF generator, mentioned previously, an infrared radiation pyrometer with integrated three-mode (PID) controller, and a data acquisition computer. A schematic layout of the temperature control system is presented in Figure 4-12. The data acquisition computer outputs an analog signal that is proportional to the desired temperature setpoint, the temperature controller compares the measured temperature value to the setpoint to determine the deviation, and a current that is proportional to the deviation is then output to regulate the power output of the RF generator. If the deviation between the measured temperature value exceeds $5 \%$ of the set-point temperature, then the controller will trigger a deviation relay to open, and shut down the high frequency generator and servo-hydraulic controller.

\subsubsection{Optical Pyrometry}

The ability for this instrument to measure temperature is based on the fact that objects emit radiant energy for which the intensity of this radiation, at a given wavelength, varies as a function of its temperature. The optical pyrometer used in the present study (IRCON Model R-99C05-0-1-0-00-0/643) utilizes two infrared detectors which compare the infrared radiation at two wavelengths and computes the ratio of the two radiation levels. This ratio serves as the output signal that the processor/indicator displays as a temperature to the user. The use of two wavelengths makes this instrument almost impervious to emissivity changes with temperature, if one assumes that whatever affects one wavelength must affect the other wavelength by the same amount ${ }^{107}$. This 
assumption is generally incorrect and a scaling coefficient, referred to as the emittance correction factor (or E-slope) by the manufacturer, must be applied to compensate for differences between the target's emissivity characteristics in its two spectral regions. Therefore, the pyrometer E-slope must be adjusted to correspond to the material under evaluation.

The optical pyrometer was located approximately $25 \mathrm{~cm}$ from the test specimen while ensuring that its viewing axis was kept perpendicular to the surfaces from which the measurement was made (see Figure 4-1). A short-range focussing lens (Model A2 - 22 $\mathrm{cm}$ to $38 \mathrm{~cm}$ range), was used for temperature measurement, which gave a spot size representative of a circular area of $4 \mathrm{~mm}$ in diameter.

The appropriate E-slope setting was determined from static temperature tests on an IN738LC calibration specimen. The first step in the calibration process was to preoxidize the calibration specimen in order to obtain a stable oxide layer. It was observed that a period of two hours at the maximum test temperature was necessary to obtain a stable temperature measurement from the infrared pyrometer. The pre-oxidizing time required for an initially unoxidized specimen was later confirmed by monitoring the extensometer feedback signal when the temperature controller was providing closed loop temperature control at the maximum test temperature. The results are presented in Figure 4-13, and clearly show that a two-hour pre-oxidizing period is necessary to obtain a stable extensometer reading. 
After pre-oxidizing, the temperature controller was configured for closed loop temperature control and the temperature was changed to the median test temperature used in this investigation $\left(850^{\circ} \mathrm{C}\right)$. The emisivity slope was then adjusted such that the temperature, indicated on the pyrometer display, matched the average temperature between the two 0.010 -inch $(0.254 \mathrm{~mm})$ diameter type- $\mathrm{K}$ thermocouples, spot welded on an IN738LC calibration specimen. The thermocouple configuration is illustrated in Figure 4-14. To assess the accuracy of the E-slope calibration, the temperature set-point was manually varied through the $750^{\circ} \mathrm{C}$ to $950^{\circ} \mathrm{C}$ temperature range used in this work in $50^{\circ} \mathrm{C}$ increments. Figure 4-15 illustrates the deviation between the average thermocouple temperature reading and that indicated by the infrared pyrometer after E-slope calibration. For the present work, an emissivity slope value of $\mathrm{L} .06$ was found to be suitable for IN738LC, and was not changed during the course of this work.

The temperature controller features a remote set-point programming option using a linearized analog input ( 0 to $10 \mathrm{VDC})$. This connection is used to change the temperature controller set-point, at timed intervals, thereby generating the temperature waveform required for TMF testing. The temperature feedback signal is available as an analog output signal ( 0 to $10 \mathrm{VDC}$ ) that is linearly proportional to the temperature indicated on the temperature display panel. This feature was used to monitor the temperature feedback in this work.

The dynamic temperature response of the pyrometer was assessed during temperature cycling trials at a temperature rate of $0.89^{\circ} \mathrm{C}$ per second. The purpose of the temperature cycling trials was to optimize the temperature controller PID tuning parameters. Before 
conducting these trials, the temperature controller response time was adjusted to the minimum setting of 10 milliseconds. Figure 4-16 illustrates the programmed temperature command and feedback response of the temperature controller based on a temperature range of $750^{\circ} \mathrm{C}$ to $950^{\circ} \mathrm{C}$ and a cycle period of 450 seconds. The maximum deviation between the programmed temperature command and feedback values was less than $5^{\circ} \mathrm{C}$, which corresponds to a phase shift of less than $5^{\circ}$, thus preserving the temperature-strain phase relationship.

\subsection{TMF and IT-LCF Testing Procedures}

The procedures outlined within the following subsections give an abbreviated list of the steps followed when conducting TMF and IT-LCF tests. For a detailed list of procedures, refer to Appendix 2. These procedures were written in the context that the appropriate actions have been taken to pre-oxidize the test specimen prior to commencing an IT-LCF or TMF test.

\subsubsection{TMF Testing Procedure}

The TMF testing procedure was as follows: First, the temperature is cycled until the specimen and extensometer reached a quasi-equilibrium thermal response ( 5 cycles were used in the present work). When commencing the fifth thermal cycle, the thermal strain response of the test specimen was digitally recorded by the data acquisition computer, as a function of cycle time. Following this, the testing machine was switched to total axial strain-control. Prior to applying mechanical deformation, the accuracy of the recorded 
thermal strain data was verified by subjecting the specimen to thermal cycling in total axial strain-control. In this case, the thermal strain compensation routine was used to compensate for the induced thermal expansion and contraction of the test specimen. Thermal cycling was continued, while compensating for thermal strain, until a quasiequilibrium thermal response was attained ( 5 cycles). Once it had been ascertained that the accuracy of the thermal strain compensation routine was satisfactory, the mechanical strain was added to the digitally recorded thermal strain data and TMF test was commenced.

\subsubsection{IT-LCF Testing Procedures}

The procedure for IT-LCF testing was as follows: First, the specimen temperature was held constant at the LCF test temperature to obtain a uniform axial temperature distribution. In this investigation, the temperature was held constant for approximately 15 minutes. Next, the testing machine was set to total axial strain control and the IT-LCF test was commenced.

\subsection{Data Acquisition System and TMF Testing Software}

The data acquisition system consisted of a National Instruments (NI) PCI-MIO-16E10 muti-function data acquisition board, NI SC2062 digital relay board, NI SCB-68 signal connection I/O terminal board housed within a NI CA-1000 signal conditioning enclosure, and a $533 \mathrm{MHz}$ personal computer with 13GB hard disk for data storage (refer

to Figure 4-1). The PCI-MIO-16E10 data acquisition board has a 16-bit, sampling analog-to-digital converter that can scan through 16 single ended or 8 differential 
channels, configured for unipolar or bipolar input, at rates up to 100,000 samples per second. The PCI-MIO-16E10 also has two double-buffered, 16-bit digital-to-analog converters that may be configured for unipolar or bipolar voltage output at rates up to 100,000 samples per second. In addition to analog $\mathrm{V} O \mathrm{O}$ capabilities, the PCI-MIO-16E10 has 8 TTL digital I/O lines that can be used to control digital devices.

\subsubsection{LabVIEW IT-LCF/TMF Testing Software}

The data acquisition and control software for TMF testing was written in LabVIEW 5 (Laboratory Virtual Instrument Engineering Workbench) graphical programming environment. LabVIEW 5 is a programming environment based on the ' $G$ ' (Graphical) programming language for use on various platforms, e.g. $x 86$, for data acquisition and instrument control. The user creates their own software modules, called virtual instruments (VI's) based on built-in library functions and custom libraries that the user can develop. Virtual instruments consist of a front panel, which the user can interact with much like a physical instrument, and a block diagram. The front panel can be customized with knobs, switches, sliders, graphs and digital display elements and it serves as the interface for setting inputs and displaying program outputs. The block diagram contains all the code to be executed within the VI and is simply a pictorial representation of the operations to be performed on input data. Code within the block diagram is executed sequentially, i.e., all input variables to an element on the block diagram are required before the element will execute. VI's are organized in a hierarchy, starting at the top-level VI, which defines the inputs and outputs for the application. Sub-VI's, which are analogous to subroutines in a text based programming language, are constructed to 
perform operations on the data as it flows through the top level VI. For example, a front panel and block diagram for a program that calculates a triangular waveform is illustrated in Figure 4-17.

The software and data acquisition system performed the following functions during a TMF or IT-LCF test:

1. Recorded total stress-strain hysteresis loops, at controlled intervals,

2. Recorded the cyclic material response, i.e. the cyclic hardening-softening behavior of the material,

3. Displayed the real-time values of relevant test parameters during a test to a user display panel, and

4. Provided timed command signals to change the temperature controller set-point, and to drive the servo-hydraulic control loop to give a controlled temperaturestrain relationship.

Presently, the testing software is limited to conducting fully reversed $(R=-1)$, strain controlled IP-OP TMF, and IT-LCF tests. When the program is run, the user can specify the type of test (IT-LCF, IP or OP-TMF), program the temperature and strain command signals (period, amplitude and offset values), select the data acquisition and analog output update intervals, specify data storage files, and specify the stress-strain hysteresis loops to record. The data acquired during a test are recorded into two ASCII text files, one for recording the cyclic hardening-softening behavior, and the other for recording the stress- 
strain hysteresis loops at user specified intervals. Depending on the type of test (IT-LCF or TMF), a third ASCII text file is used to record the thermal strain response of the specimen.

Four analog input channels are used to acquire temperature, total strain and load data, and monitor the interlock sense connection during a test. During a fatigue test, all the major variables (temperature, total strain and load) can be monitored in real time on the software front panel. As shown in Figure 4-18, the front panel displays the mechanical stress-strain hysteresis loop, plotted as load (volts) versus mechanical strain (volts), which is updated at the end of each test cycle. Here, the mechanical strain was determined by subtracting the thermal strain recorded during cycling temperature at zero stress. A group of digital indicators shows the real time values of load (volts), strain (volts) and specimen temperature $\left({ }^{\circ} \mathrm{C}\right.$ ). A control is provided for the user to specify a load value, in volts, which is used to stop a test if the fatigue failure is based on specific drop in peak tensile load (stress) amplitude. A graph on the front panel, which shows the peak tensile load (volts) versus cycles, is useful for monitoring the cyclic behavior of the test material. The user can also specify the deformation data (hysteresis loop) recording interval by a control on the front panel.

Two analog output channels are used to generate the temperature and total strain command signals used to change the temperature controller set-point and command the load-unit controller (when in total axial strain control). The testing software controls the generation (update rate) of the temperature and total axial strain command waveforms. Two digital outputs are used to control the SC2062 digital relay board for the PC- 
interlock and induction heater run/stop connections. The analog input/output, and digital channel configuration is given in Appendix 1.

For a detailed explanation of the operation of the TMF testing software and the program source code refer to Appendix 1. User intervention is necessary to configure the test software prior to commencing an IT-LCF or TMF test. A detailed procedure for configuring the software is given in Appendix 2.

\subsubsection{Noise Considerations for Analog Signals}

The use of RF induction heating often introduces a significant amount of electrical noise in analog voltage signals. Several means of mitigating the effects of RF noise are:

1. Signal conditioning hardware, i.e. low-pass filtering and amplification,

2. Observing proper grounding and shielding techniques,

3. Calculating the average of a number of measurements,

4. Using software based digital signal filtering techniques, and

5. Proper data acquisition board configuration and signal connection.

The last point listed above (5), is the easiest means for mitigating the effects of RF noise. Jaffar-Shah ${ }^{108}$ recommends coaxial cable for applications where the primary source of noise has a frequency greater than $100 \mathrm{kHz}$ because of the cable's lower capacitance and more uniform characteristic impedance. Following the recommendation of Jaffar-Shah, coaxial cables were used for signal connections to the temperature and load-unit 
controllers. Besides using recommended cabling for analog signals, the analog input channels were configured for differential mode signal connections. The primary motivation for configuring the DAQ card for differential signal connections is that most of the noise, present as a common-mode voltage ${ }^{108}$, is rejected by the DAQ card amplifier.

An averaging scheme was also implemented in the LabVIEW TMF testing software to reduce analog signal noise. Loewenstein ${ }^{109}$ and Donald ${ }^{\mathrm{Il} 0}$ illustrated that for independent and identically distributed readings uncertainty in a measurement can be reduced by $\sqrt{1 / n}$ where $\mathrm{n}$ is the number of measurements that were averaged. The noise in the analog feedback signals measured by the data acquisition system used in this work was investigated. Figure 4-19 (A) illustrates the noise present in a series of data when averaging 80 data points, and Figure 4-19 (B) illustrates the noise reduction for various over-sampling rates. For the present study 80 data points were averaged per reading.

One additional measure taken in the present work to mitigate the effects of noise in the analog signal readings was to select the minimum strain and load cell ranges to accommodate the total strain and loads imposed during a TMF or IT-LCF test. Selecting the minimum strain and load ranges maximizes the signal-to-noise ratio. By using the forgoing techniques of removing analog signal noise, the noise in analog signal measurements was reduced to a less than $2 \mathrm{mV}$ peak-to-peak. 


\subsection{Data Analysis Procedures}

TMF and IT-LCF tests generated a considerable amount of data, which required postprocessing. A Visual Basic (VB version 6) program was written specifically to facilitate this task. The output files from the VB post-processing program were in ASCII text, and formatted specifically for importing into Tecplot'. The post-processing program generated two output files. The first file contained the cyclic response of the specimen during a test. The second file contained the total, mechanical, inelastic, and elastic stressstrain hysteresis loops. A complete program listing is given in Appendix 3.

\subsubsection{Hysteresis Loop Components}

As described previously, the actual strain component measured by the axial extensometer was the total strain, i.e. the sum of mechanical and thermal strains. In the present work, the thermal strain component, digitally recorded prior to the test, was subtracted from the total axial strain measured by the axial extensometer to determine the stress-mechanical strain hysteresis loop.

For an IT-LCF test, the elastic strain component of the hysteresis loop was determined from the slope of the hysteresis loop, which gave the elastic modulus of the material at the test temperature. The slope of the TMF hysteresis loop cannot be used to determine the elastic modulus of a material subjected to thermal mechanical deformation because

${ }^{r}$ Tecplot $^{\circledast}$ - Amtec Engineering, Inc. Bellevue, WA $98009-3633$ 
the temperature is continuously changing during the deformation process. To obtain the inelastic stress-strain hysteresis loop the following equation was used for TMF test data:

$$
\varepsilon_{\text {in }}=\varepsilon_{\text {mech }}-\frac{\sigma}{E(T)}
$$

where $\varepsilon_{\text {in }}$ was the inelastic strain, $\varepsilon_{\text {mech }}$ was the mechanical strain component, $\sigma$ was the applied stress, and $E(T)$ was the elastic modulus of the material as a function of temperature. The relationship between elastic modulus and temperature must be determined prior to commencing a TMF test.

\subsubsection{Determining the Temperature Dependent Elastic Modulus}

Prior to commencing a TMF test, the temperature dependent elastic modulus was measured using the guidelines given in the ASTM standard, E 111-82 ${ }^{11}$. It was important to determine the elastic modulus for each specimen, since cast superalloys tend to exhibit anisotropic behavior ${ }^{22}$. In the case of TMF tests, the elastic modulus was necessary to reduce the mechanical strain component of the stress-strain hysteresis loop to elastic and inelastic components. In this work, the elastic modulus was determined in $50^{\circ} \mathrm{C}$ increments through the test temperature range $\left(750^{\circ} \mathrm{C}\right.$ to $\left.950^{\circ} \mathrm{C}\right)$. The elastic modulus was measured with the testing machine in force control using a fully reversed triangular load function with a frequency of $0.01 \mathrm{~Hz}$. The amplitude of the triangular waveform was changed for each temperature increment, such that the applied load was sufficiently low as to not exceed the proportional limit. Three elastic modulus measurements were made at each temperature and the average of the three measurements was used to determine the 
elastic modulus-temperature relationship. A second order polymomial was fitted to the elastic modulus data, from each temperature increment, by the least squares method. Figure 4-20 illustrates a typical elasitc modulus versus temperature plot obtained from this work.

\subsubsection{Determination of Failure}

For the present work, failure was defined as a 5\% drop in the stabilized peak tensile stress amplitude. In several cases, specifically with IP-TMF tests, a subtle cyclic tensile load drop was observed. When this was observed, fatigue life was defined as a $5 \%$ drop in tensile stress amplitude the value arrived at by the intersection point of a line tangent to the region where rapid tensile stress drop occurred and the line tangent to the cyclically uniform region (see Figure 4-21).

\subsubsection{Presentation of Results}

The standard means of presenting LCF and TMF life data is to plot total strain range versus life on a log-log graph. The elastic and inelastic components of strain can be extracted from the stress-mechanical strain hysteresis loop and plotted on the strain-life curve as well. A plot of inelastic strain range versus life for many materials is log-linear. The log-linear relationship for the present work was determined using the statistical analysis procedures given in ASTM E $739^{112}$. 


\begin{tabular}{|c|c|}
\hline Strain Gage Specimen Position & \% Bending Strain \\
\hline $0^{\circ}$ & 4.52 \\
\hline $90^{\circ}$ & 10.47 \\
\hline $180^{\circ}$ & 4.60 \\
\hline $270^{\circ}$ & 5.50 \\
\hline $0^{\circ}$ & 4.33 \\
\hline
\end{tabular}

Table 4-1 - Bending strain readings taken before the commissioning test program using 12 strain gage specimen and $5 \mathrm{kN}$ axial load. 


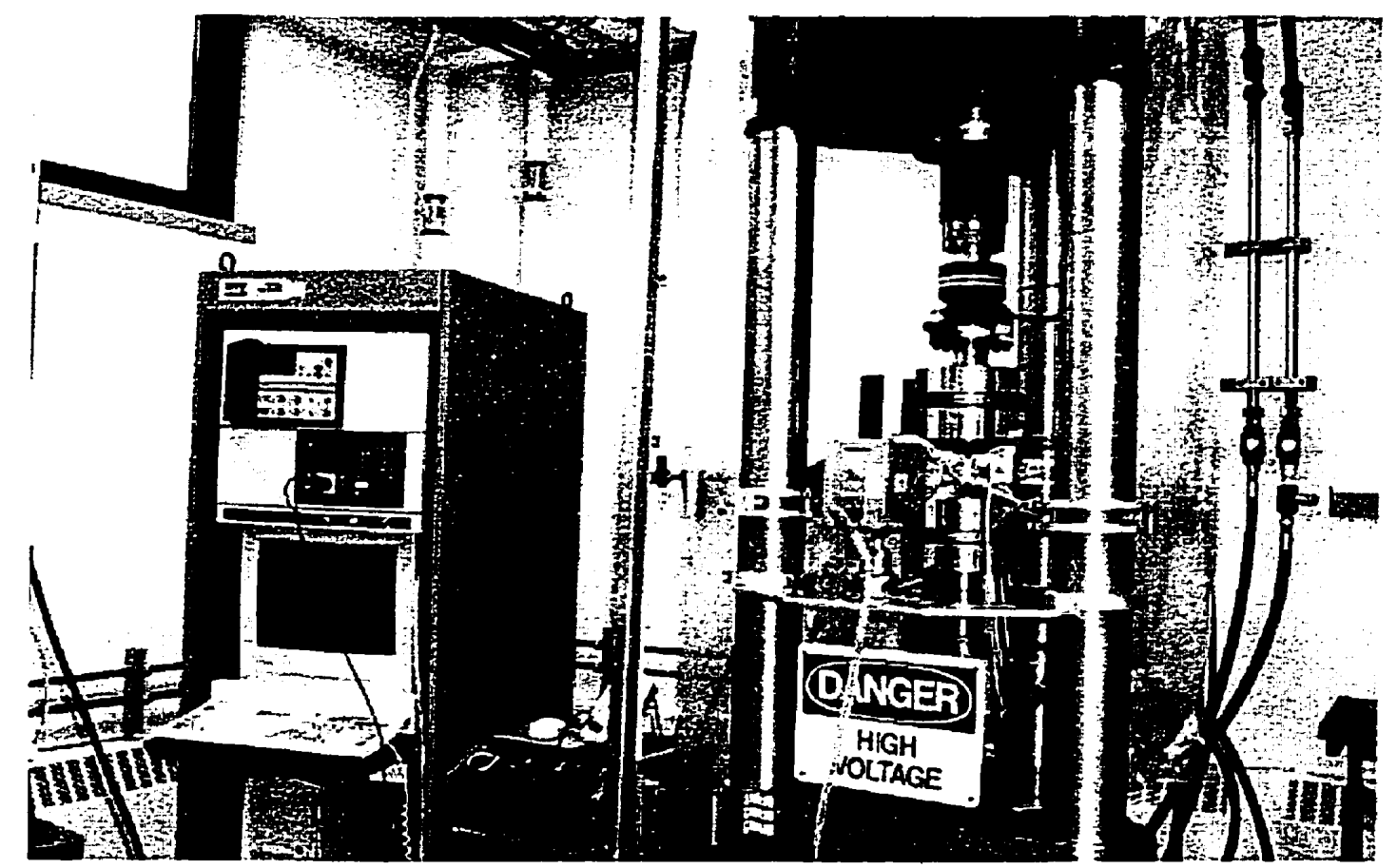

Figure 4-I - General layout of the IAR-NRC thermal-mechanical fatigue testing facility.

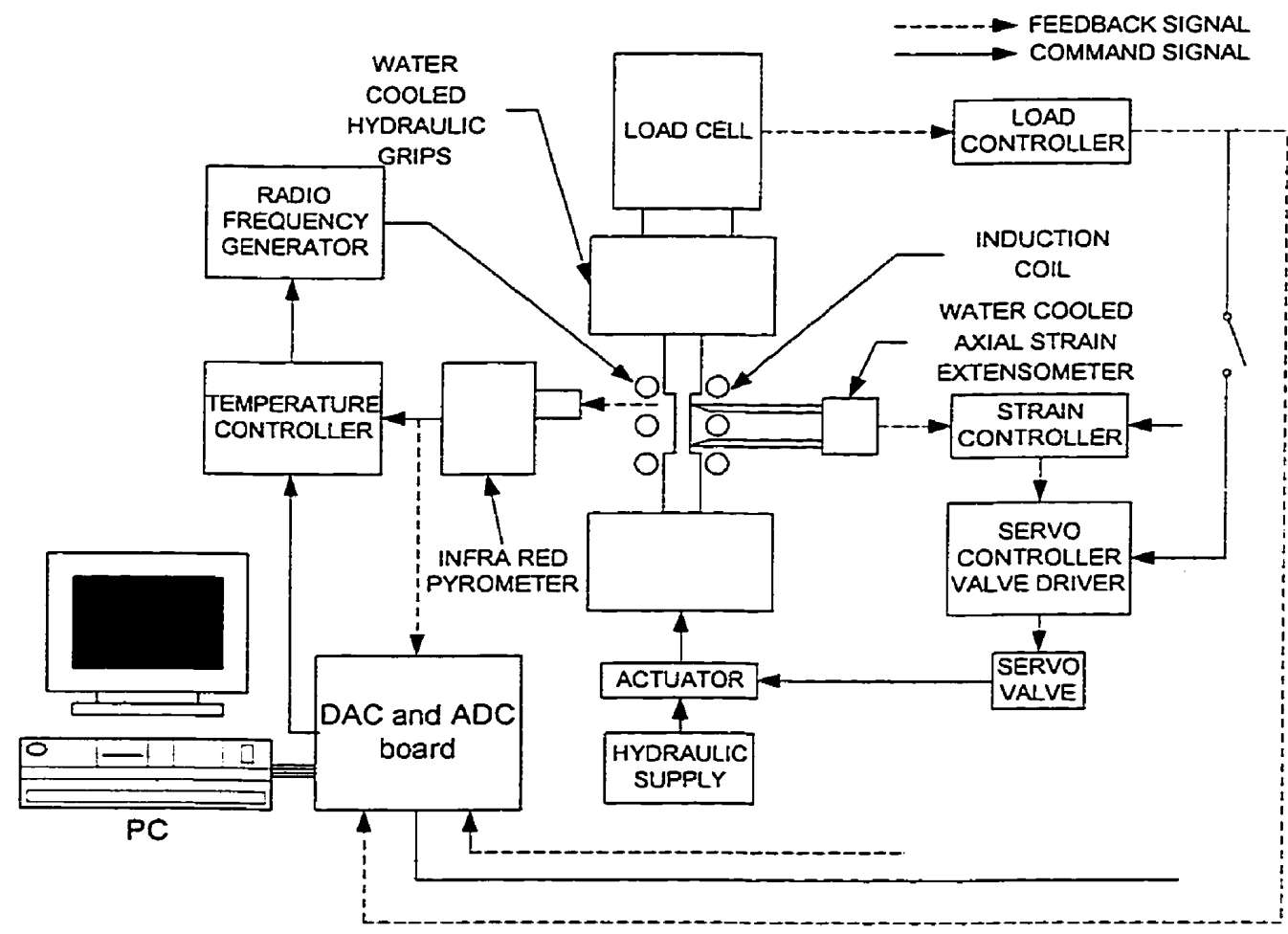

Figure 4-2 - Control block diagram for the TMF testing facility illustrating the temperature and total axial strain control loops. 


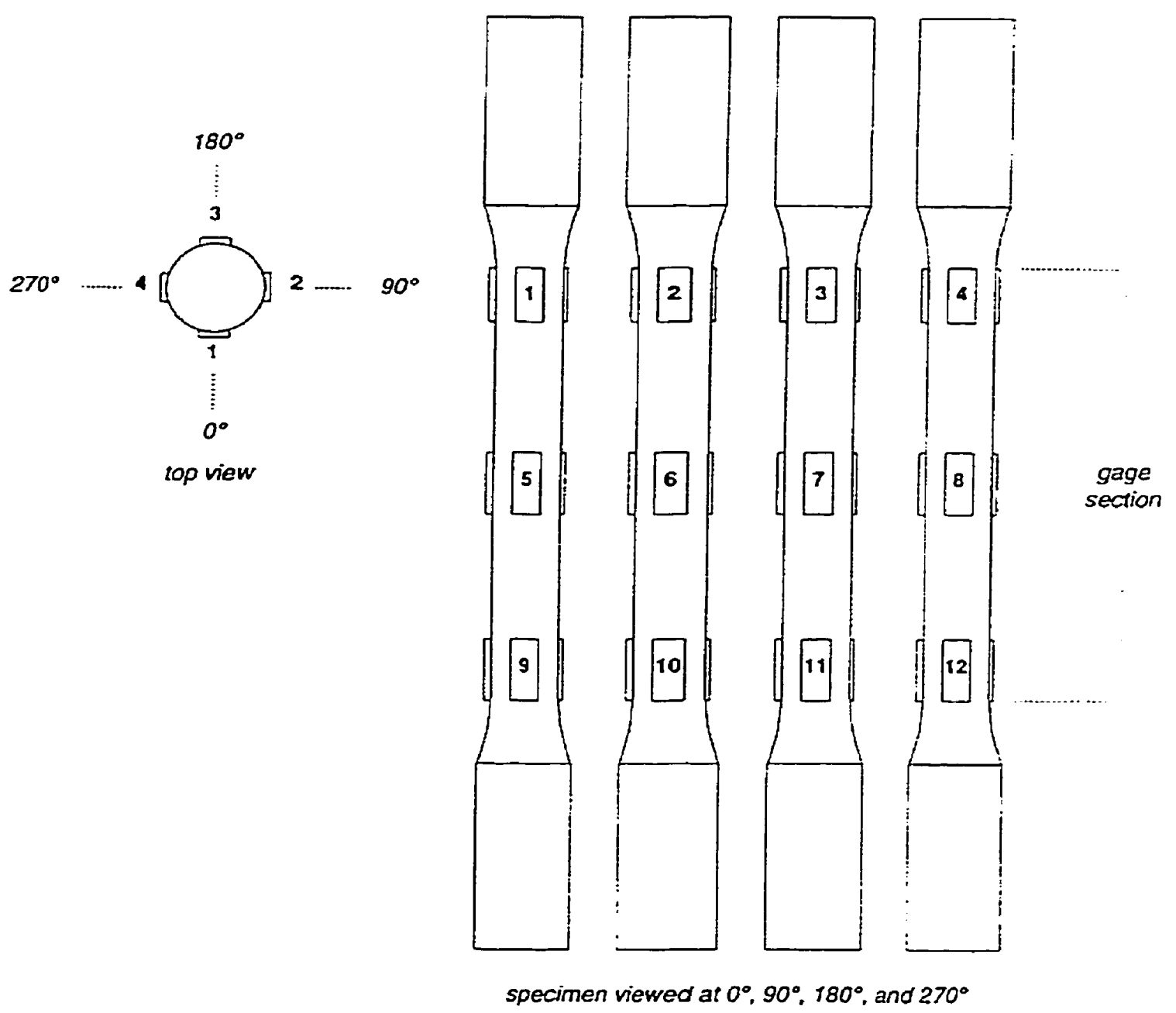

Figure 4-3 - Strain gage alignment specimen for setting the concentric and angular alignment of the load train. 


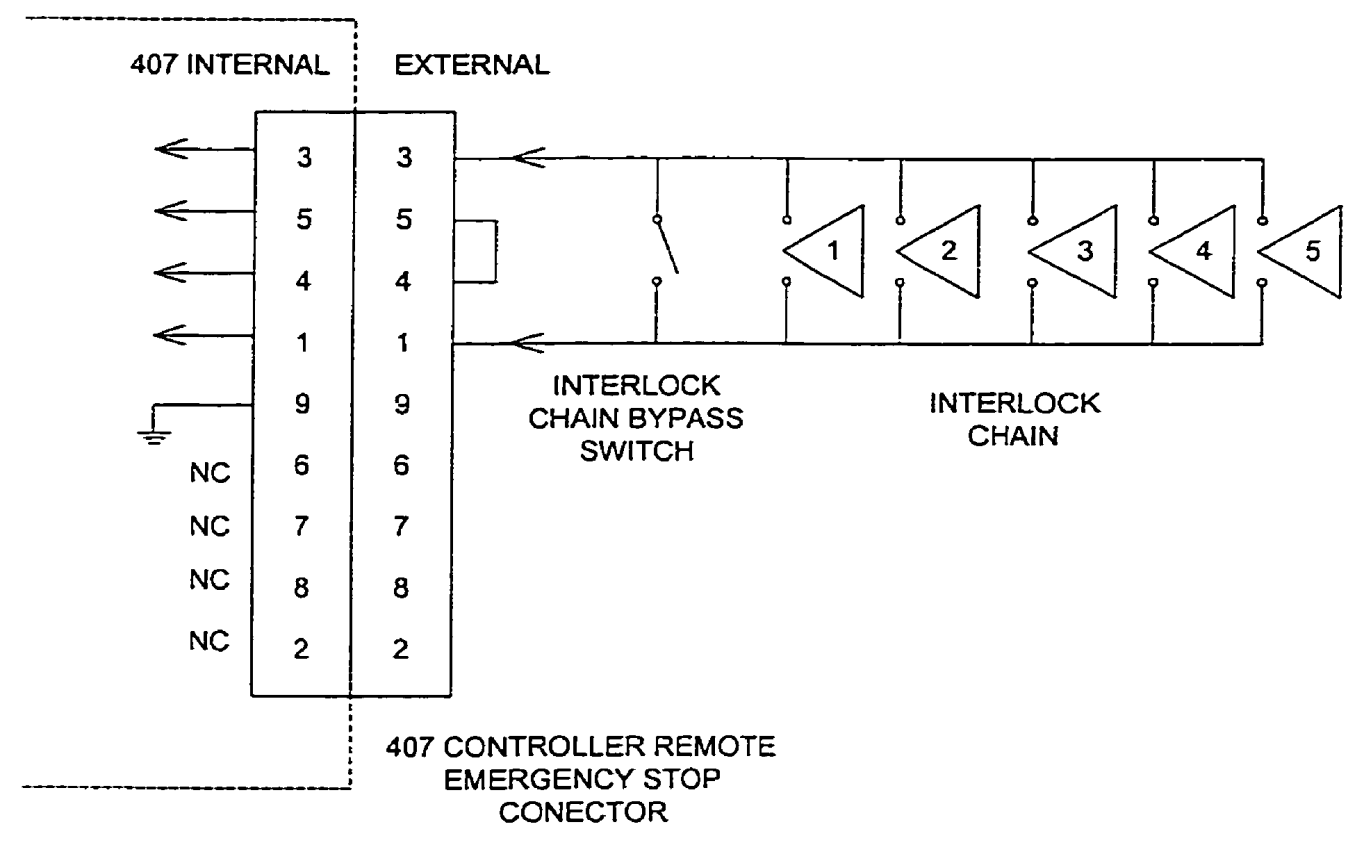

Figure 4-4 - Interlock chain connected to MTS 407 controller remote emergency stop connector.

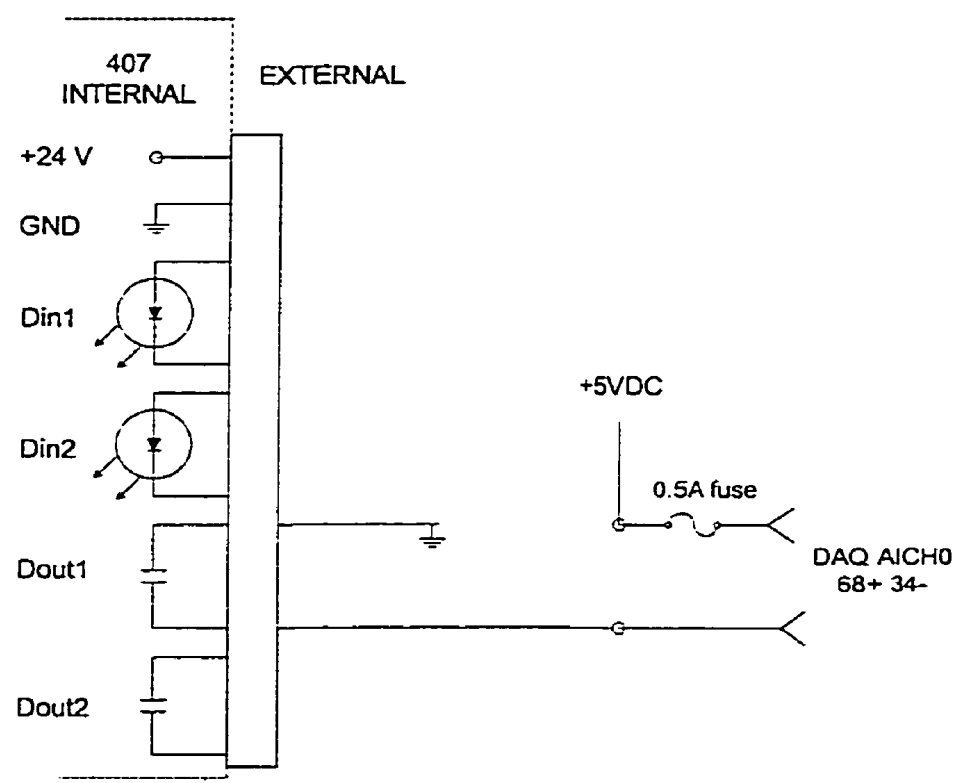

Figure 4-5 - Schematic view of PC/MTS 407 controller interlock sense connection. 


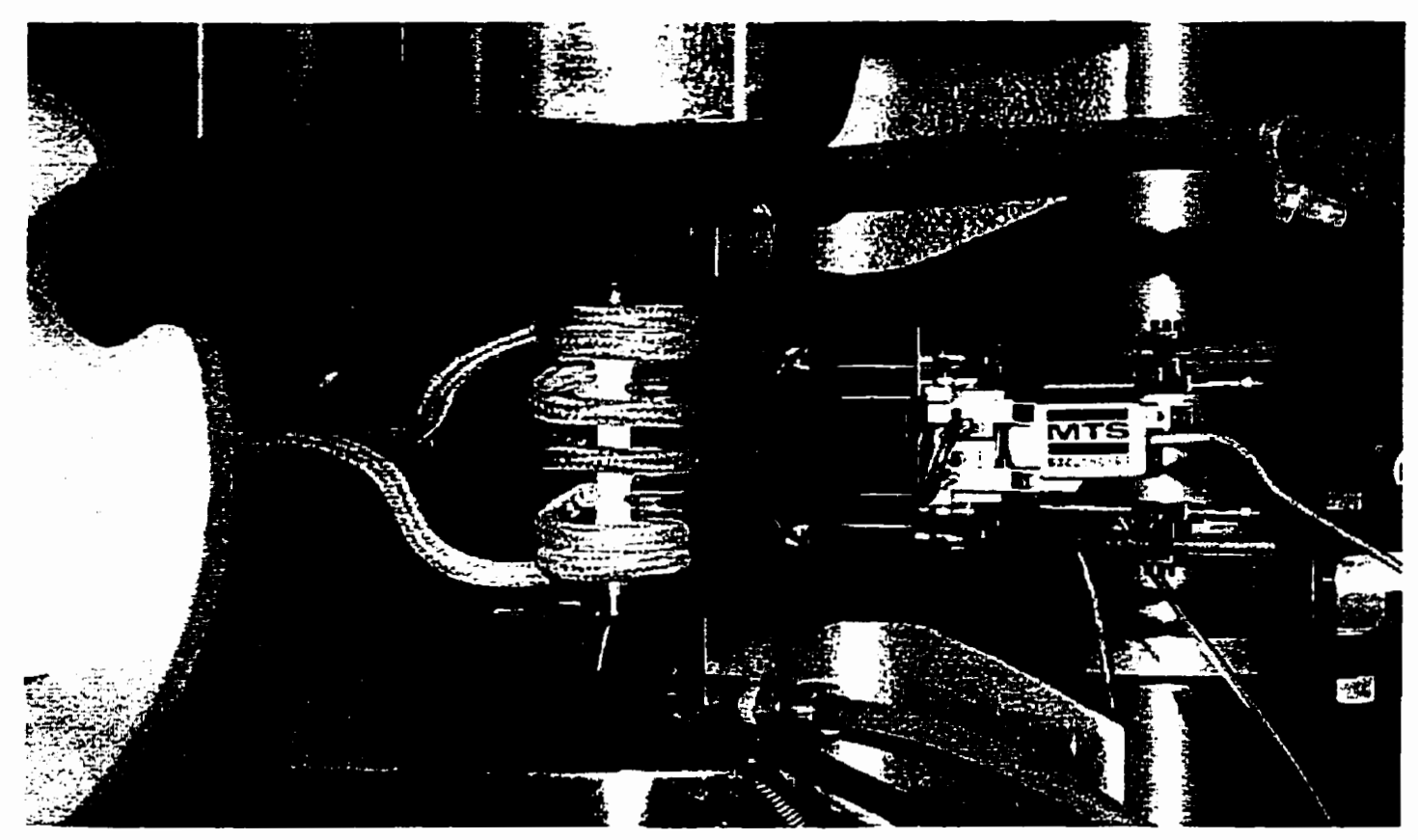

Figure 4-6 - Close-up view of $25 \mathrm{~mm}$ high temperature axial extensometer and induction coil used for isothermal LCF testing.

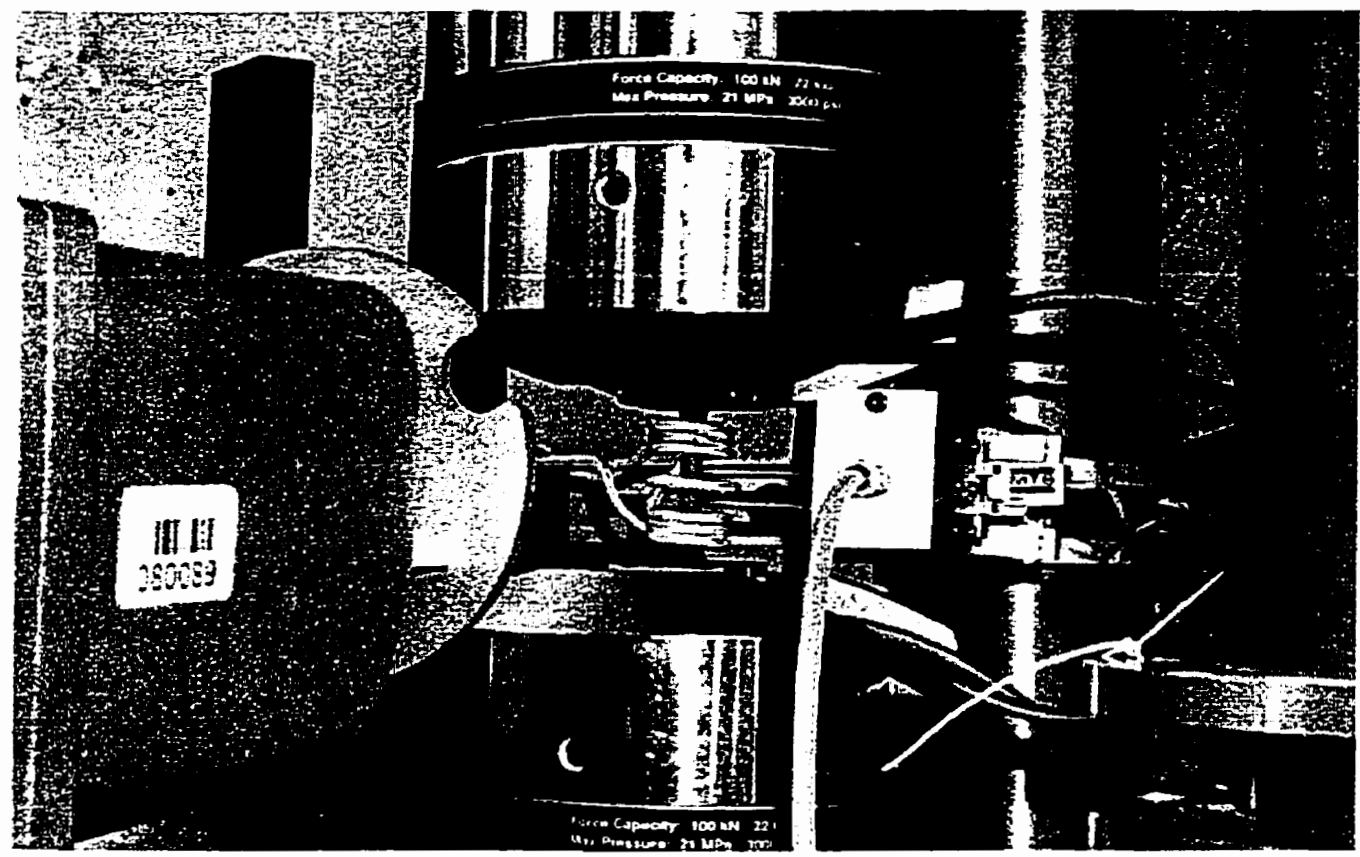

Figure 4-7 - Close-up view of radiation pyrometer sensing head, $12 \mathrm{~mm}$ high temperature axial extensometer and induction coil for TMF testing. 


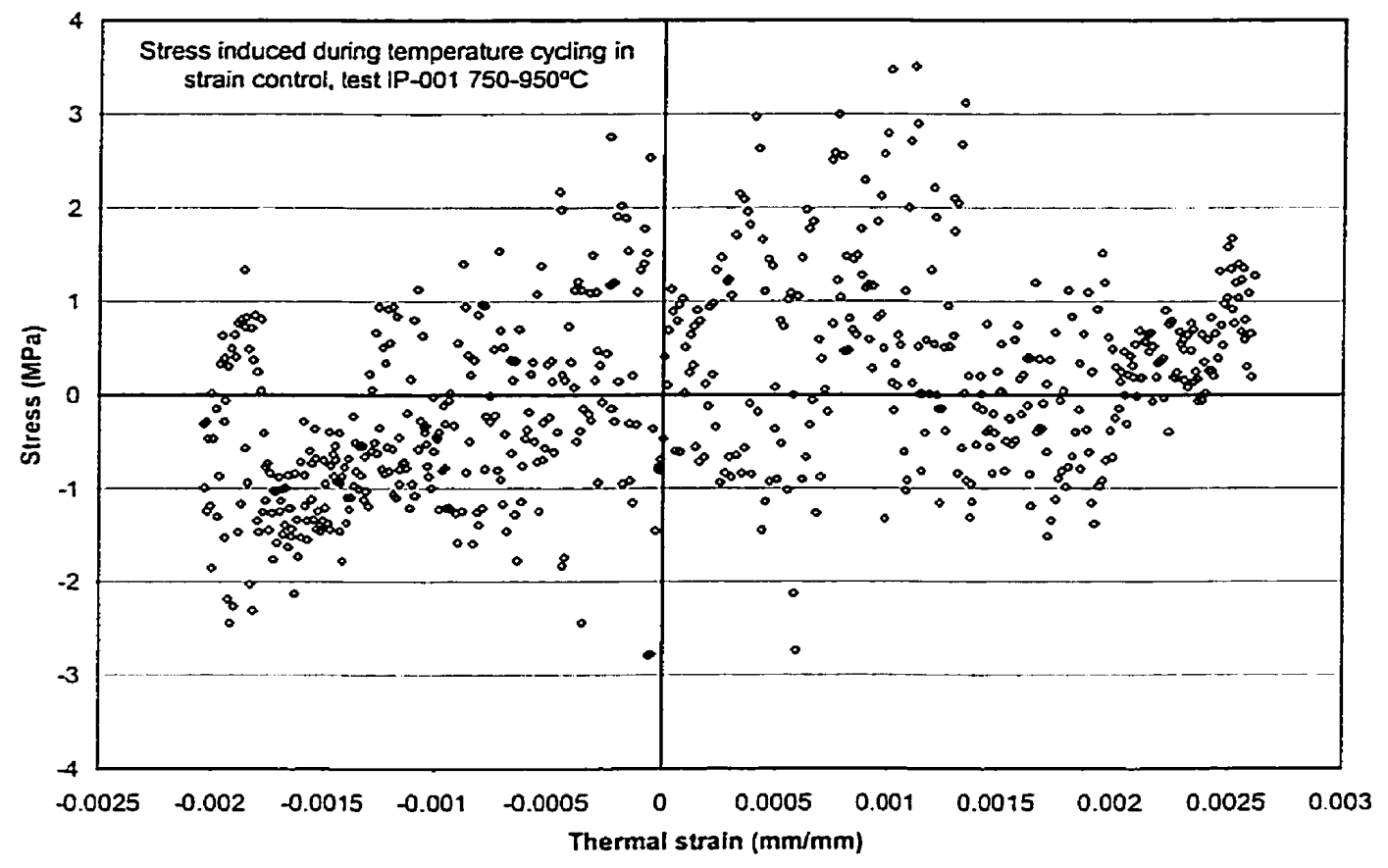

Figure 4-8 - Stress induced while compensating for thermal strain during thermal cycling.

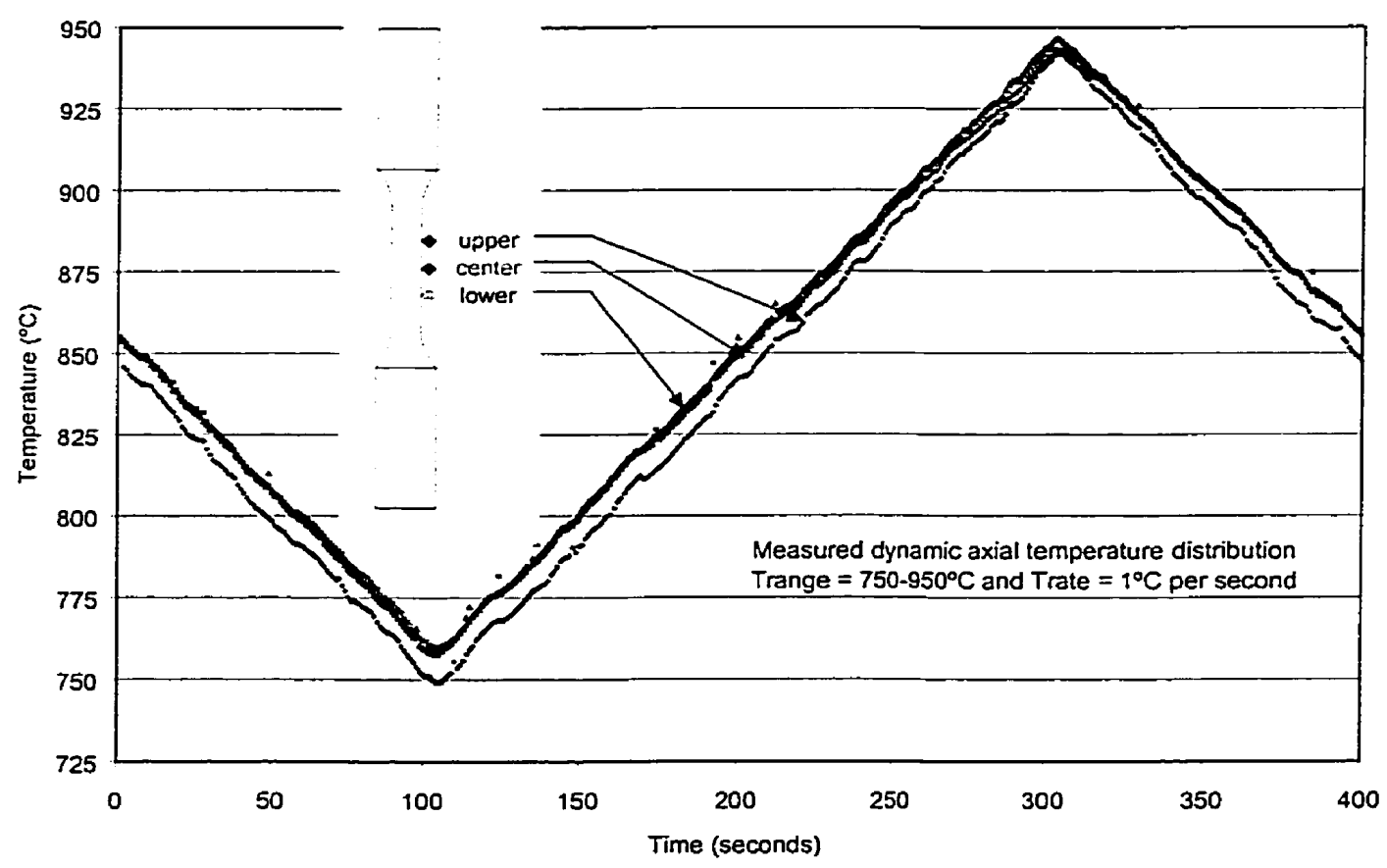

Figure 4-9 - Measured dynamic axial temperature distribution for $750^{\circ} \mathrm{C}$ to $950^{\circ} \mathrm{C}$ temperature range and temperature rate of $1^{\circ} \mathrm{C}$ per second. 


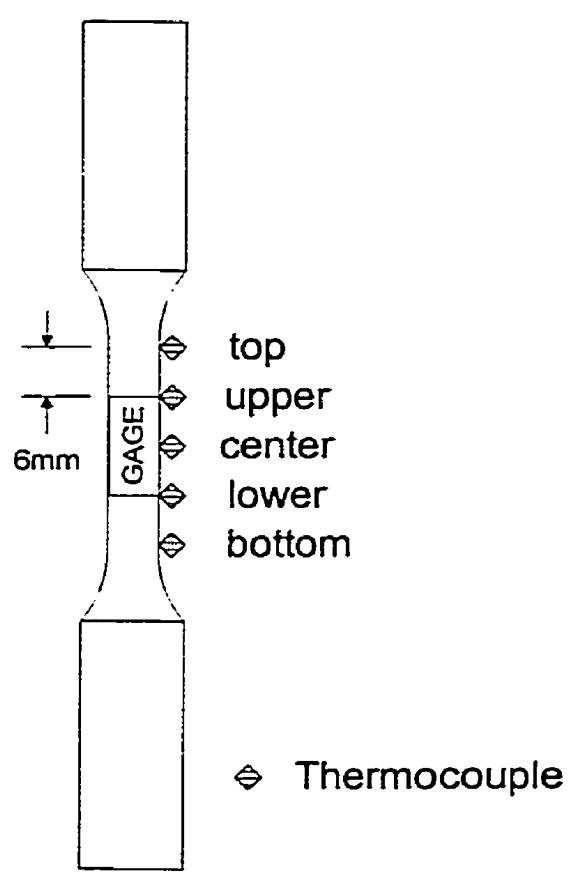

Figure 4-10 - Thermocouple configuration for determining axial temperature gradients.

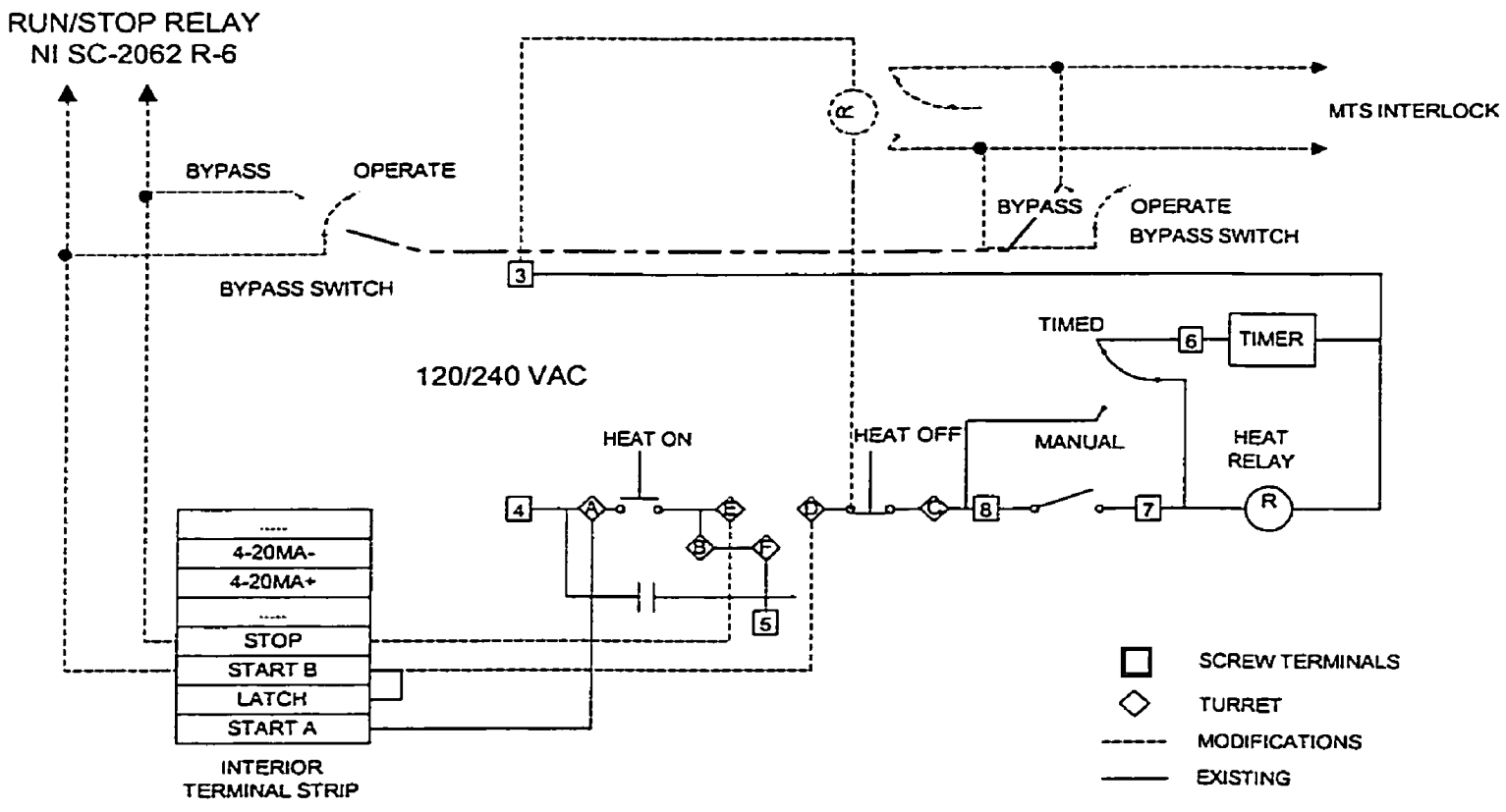

Figure 4-1 1 - Modifications to induction heater heat-on/off panel to provide an interlock connection to the MTS 407 controller and run/stop connection to the data acquisition PC. 


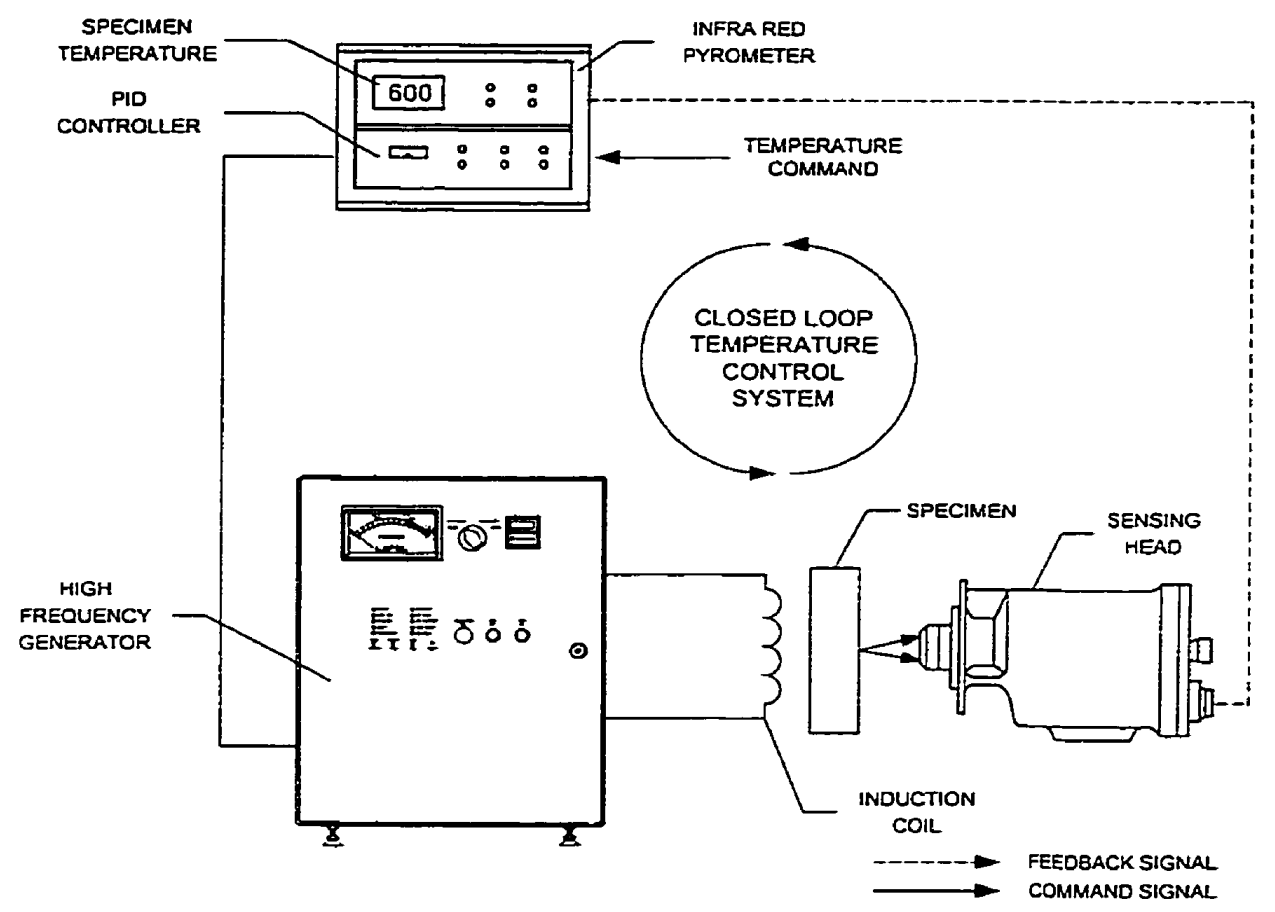

Figure 4-12 - Schematic view of the closed-loop temperature control system.

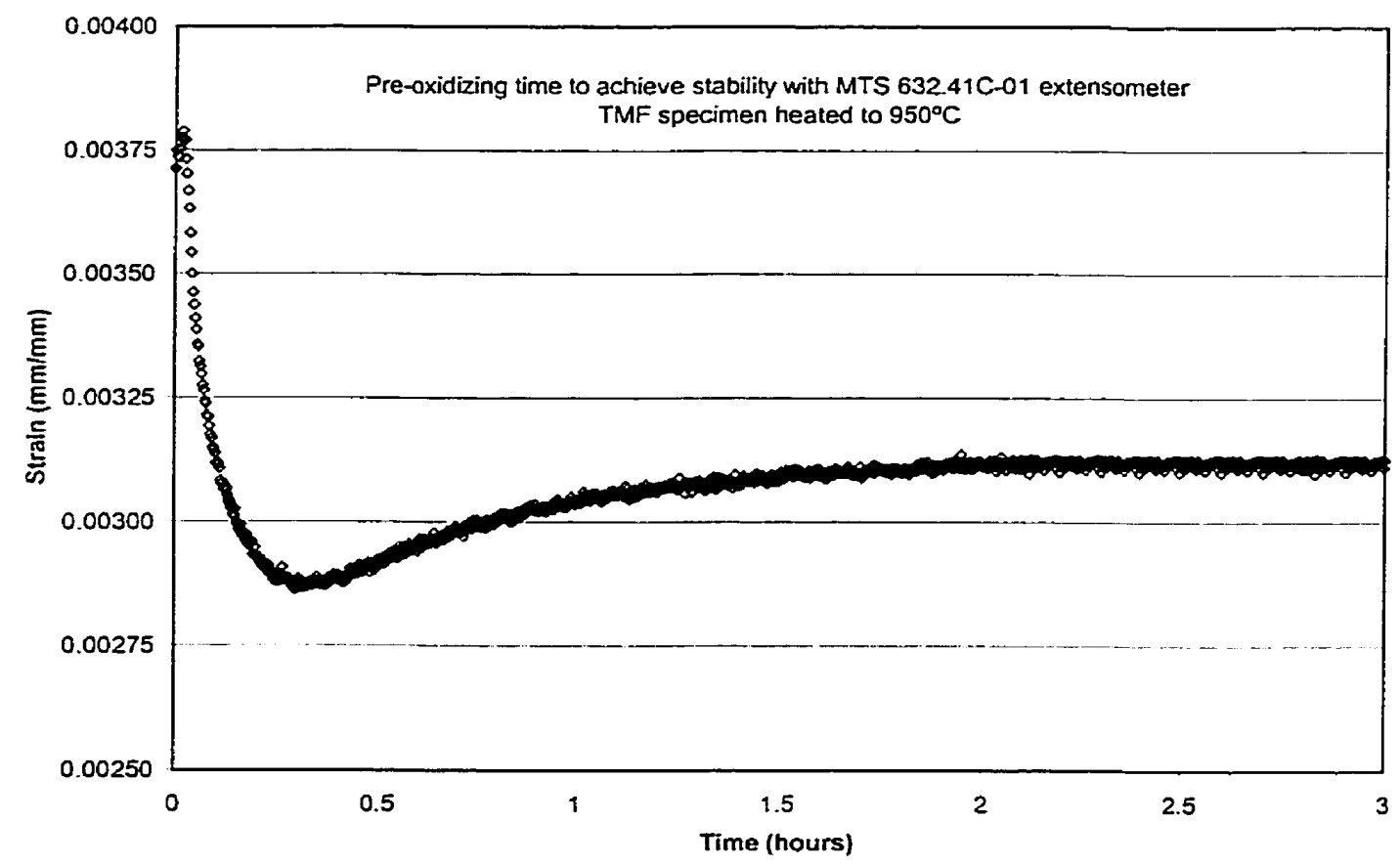

Figure 4-13 - Plot illustrating the drift observed in the extensometer feedback signal when an IN 738LC specimen was heated to $950^{\circ} \mathrm{C}$ and held constant for 3 hours. 


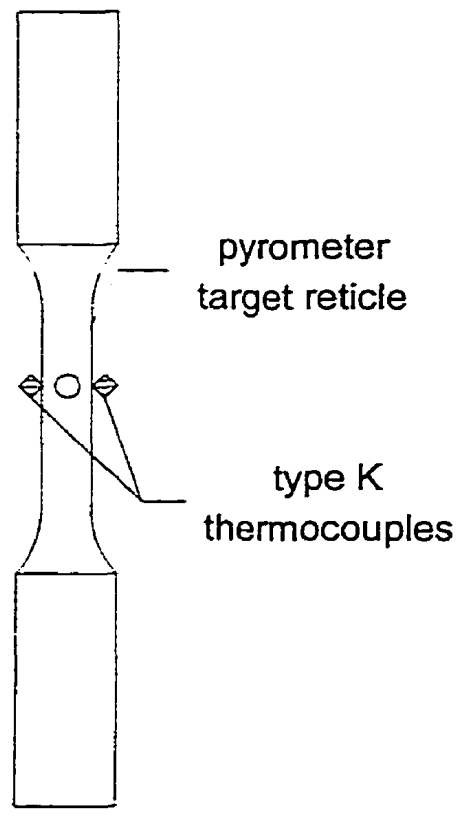

Figure 4-14 - Thermocouple setup for calibrating the infrared pyrometer E-slope.

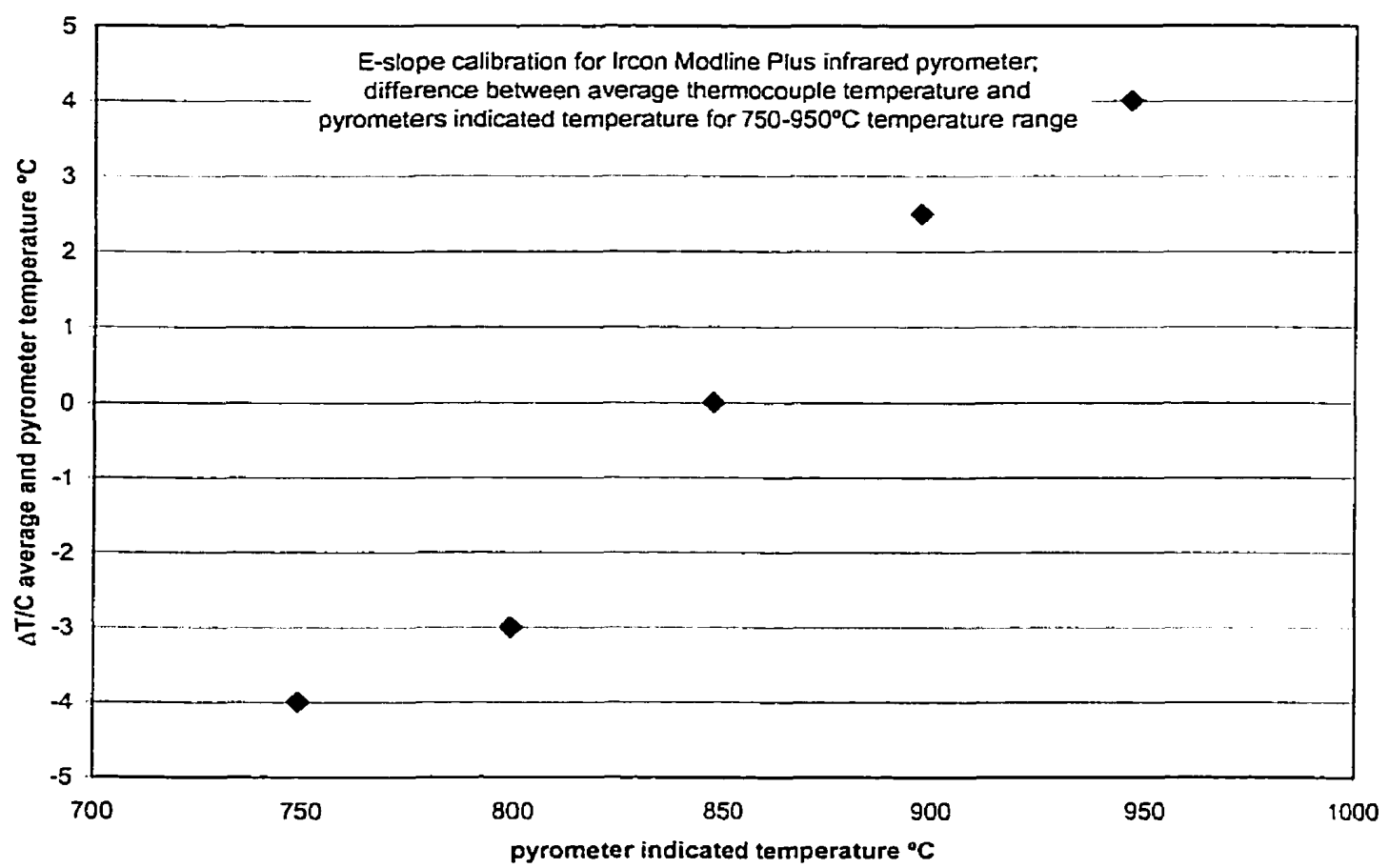

Figure 4-15 - E-slope calibration curve for the infrared pyrometer used in the present study, showing the difference between the average thermocouple temperature reading and the temperature indicated by the infrared pyrometer. 


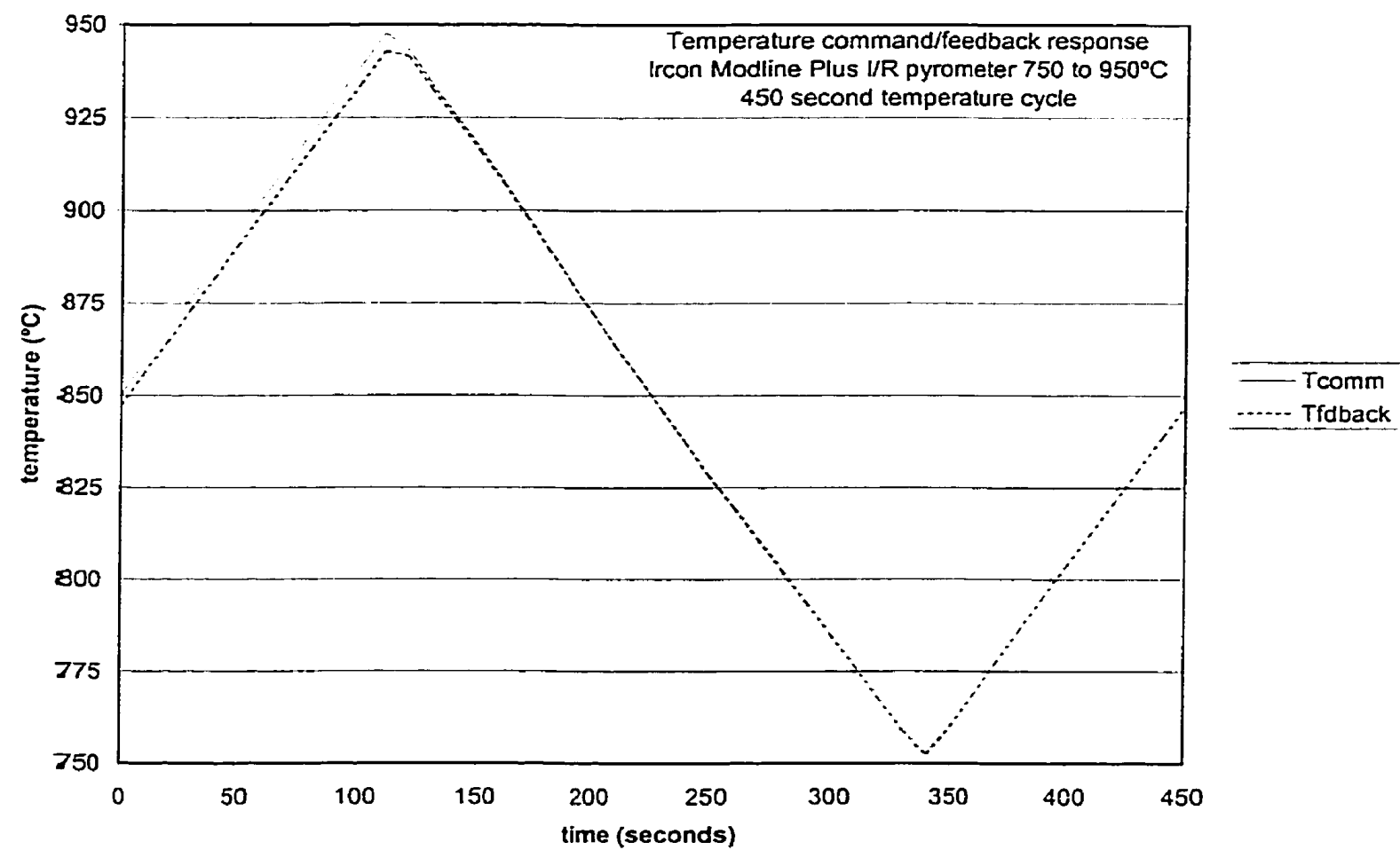

Figure 4-16 - Temperature command and feedback response of the infrared pyrometer temp-erature controller for a temperature range of $750^{\circ} \mathrm{C}$ to $950^{\circ} \mathrm{C}$ and a cycle period of 450 seconds. 


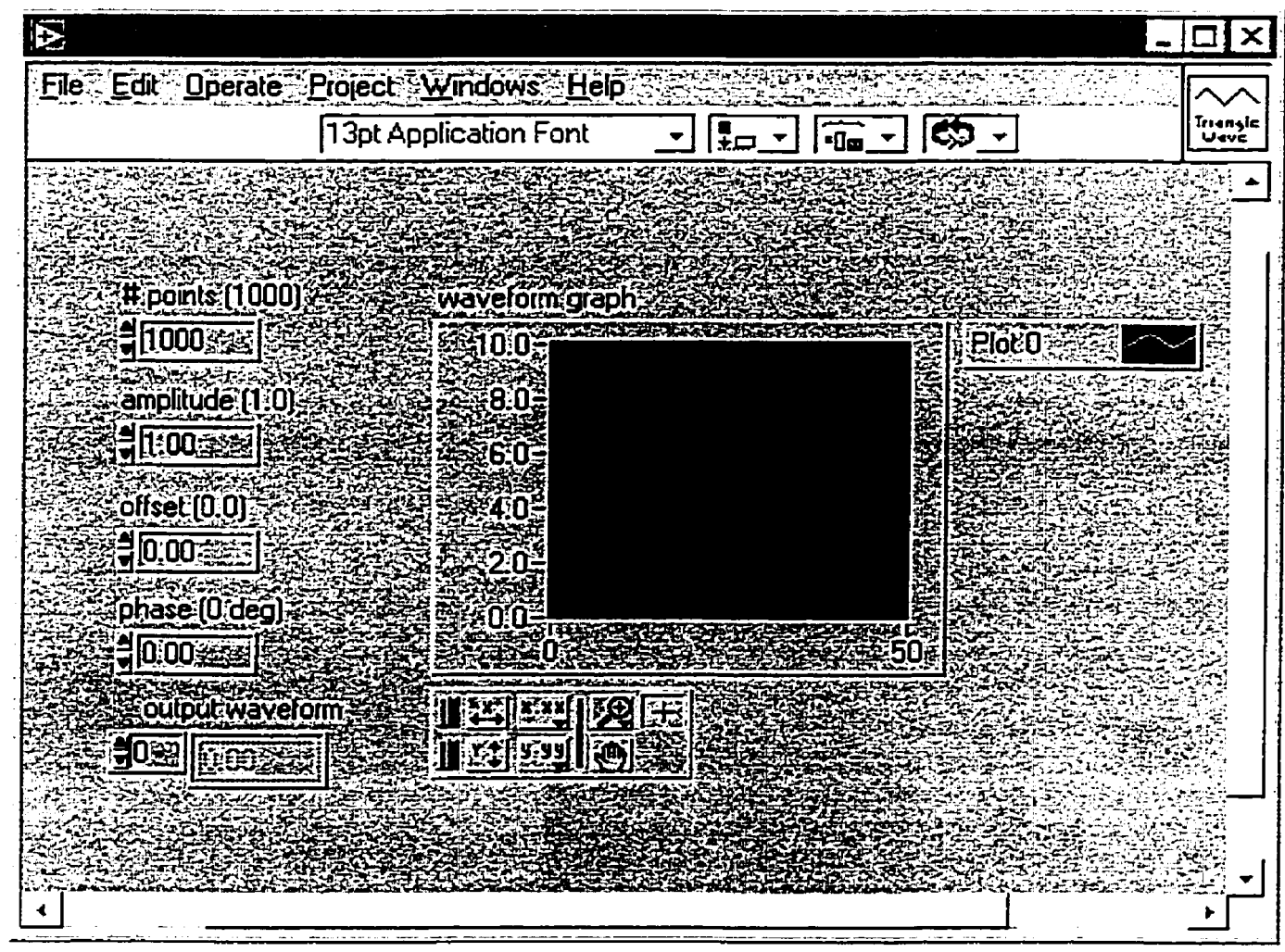

A)

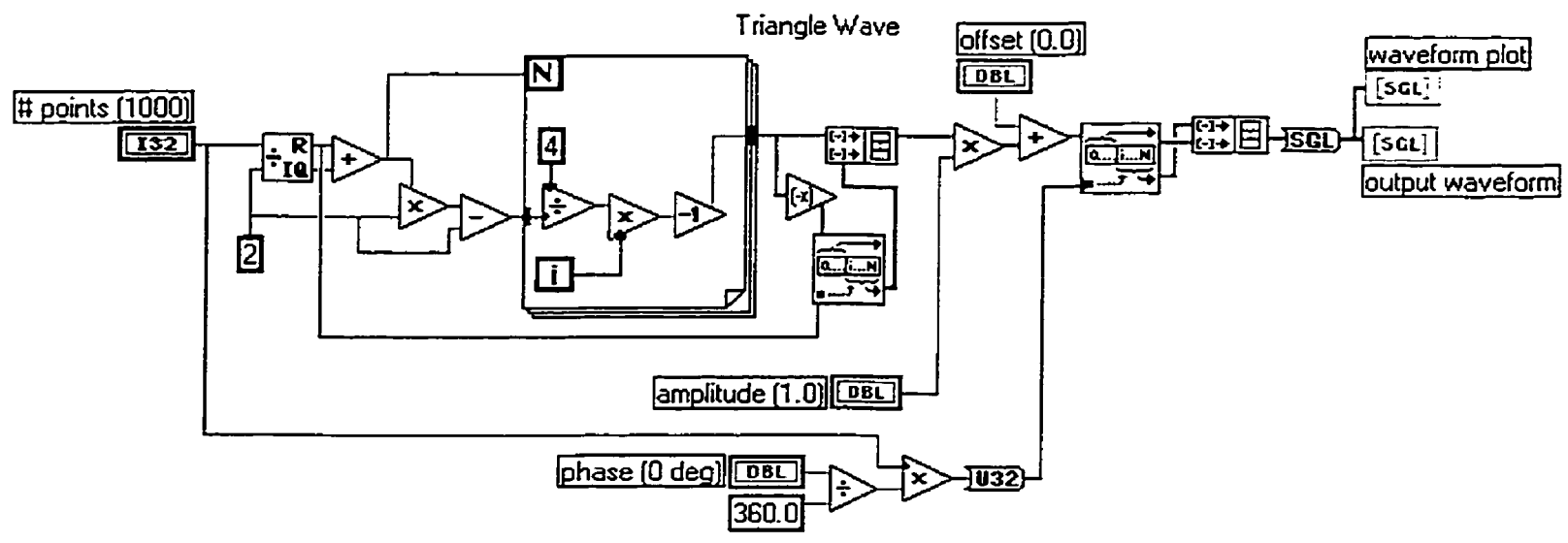

B)

Figure 4-17 - Example of a LabVIEW front panel (A) and block diagram (B) for calculating a triangular waveform. 


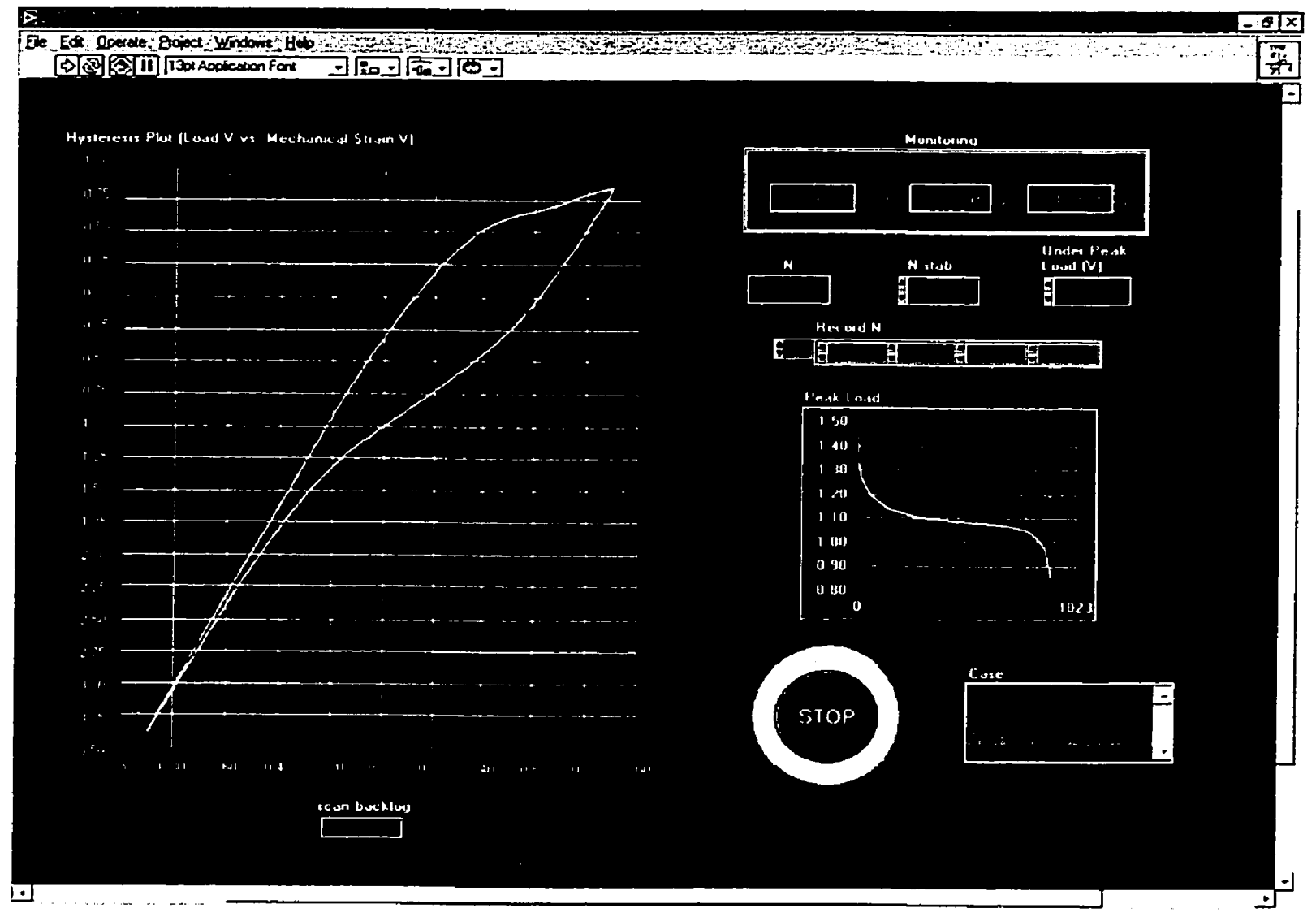

Figure 4-18 - User display screen for LabVIEW TMF testing software during a test. 


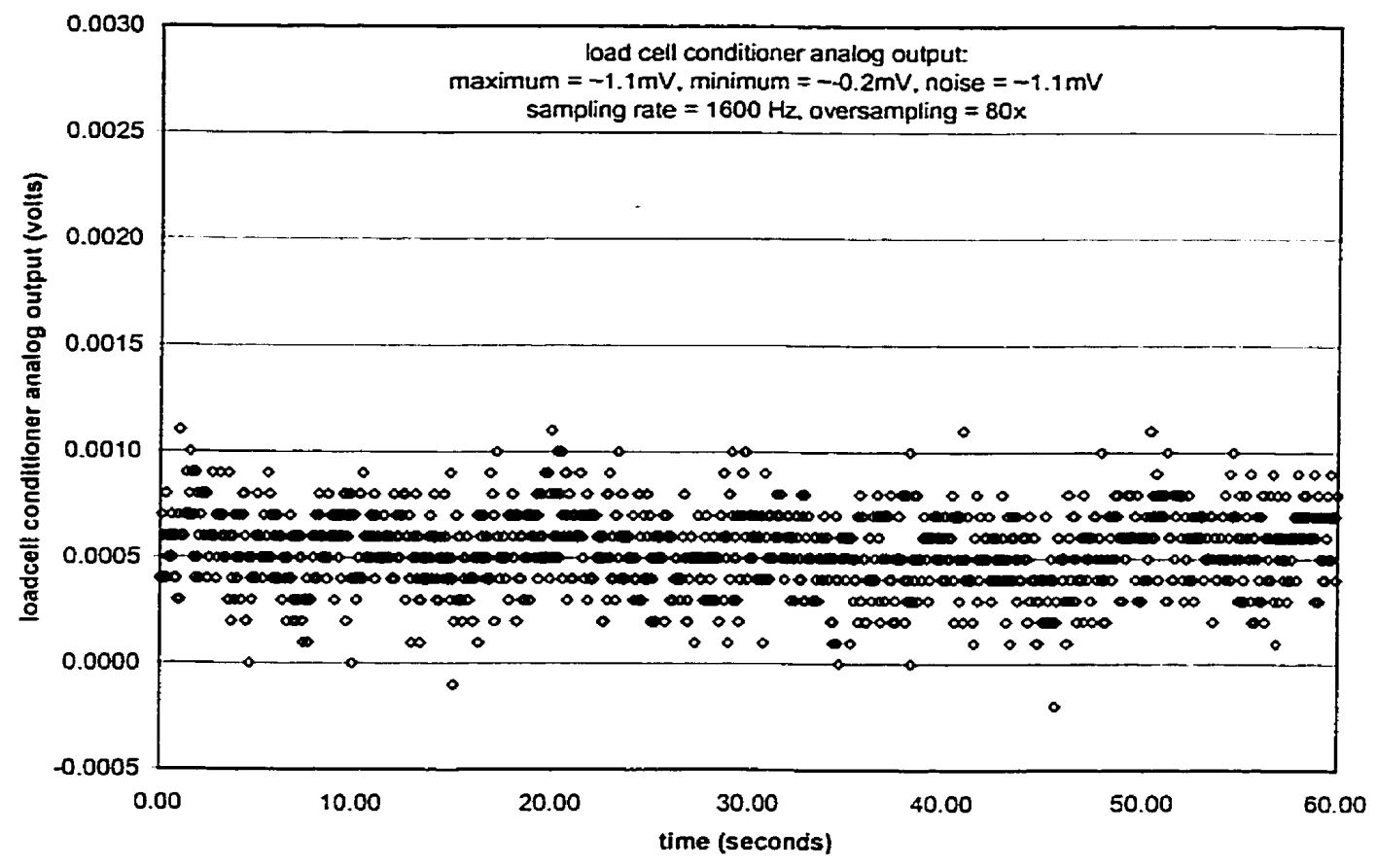

(A)

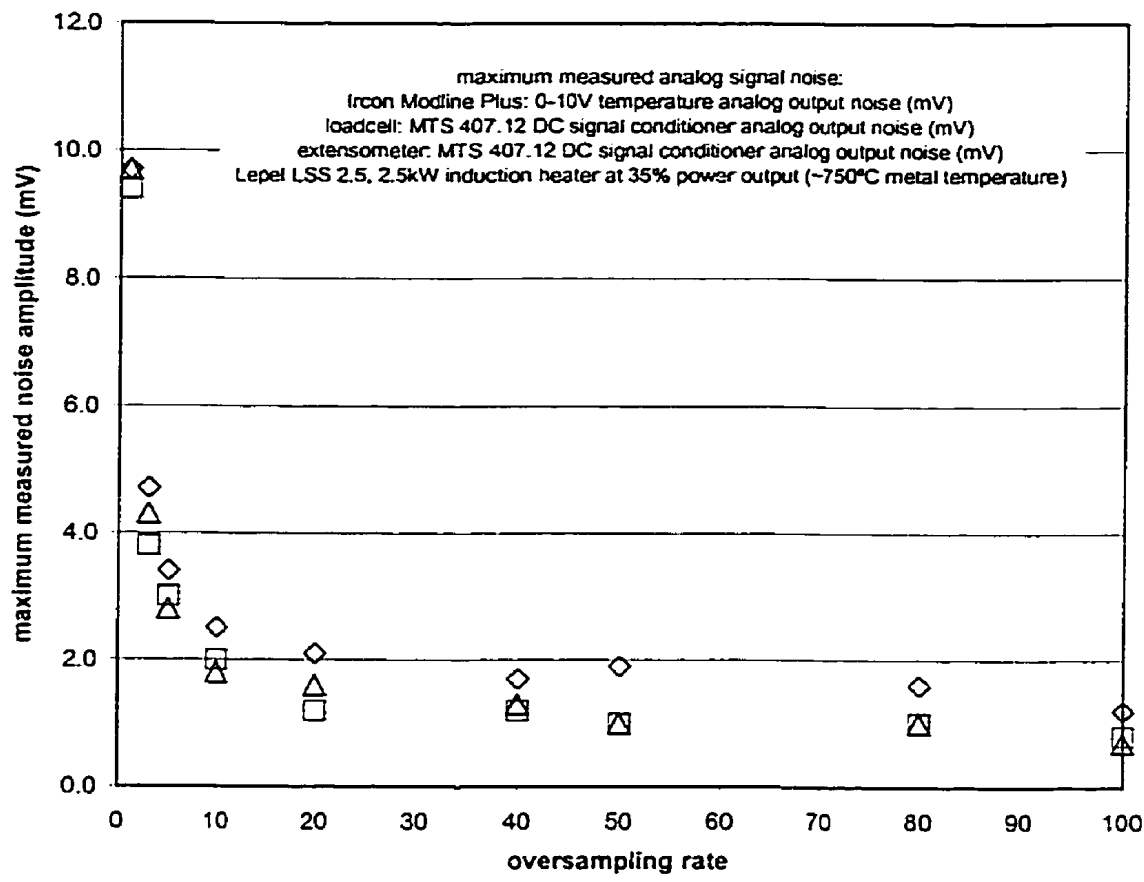

$\diamond$ Temperature (mV) ¿Strain (mv) $\Delta$ Load (mV)

Figure 4-19 - Analog signal noise from the loadcell signal conditioner after averaging 80 readings (A) and the maximum peak-to-peak signal noise as a function of the number of samples averaged for the temperature, extensometer and loadcell feedback signals (B). 


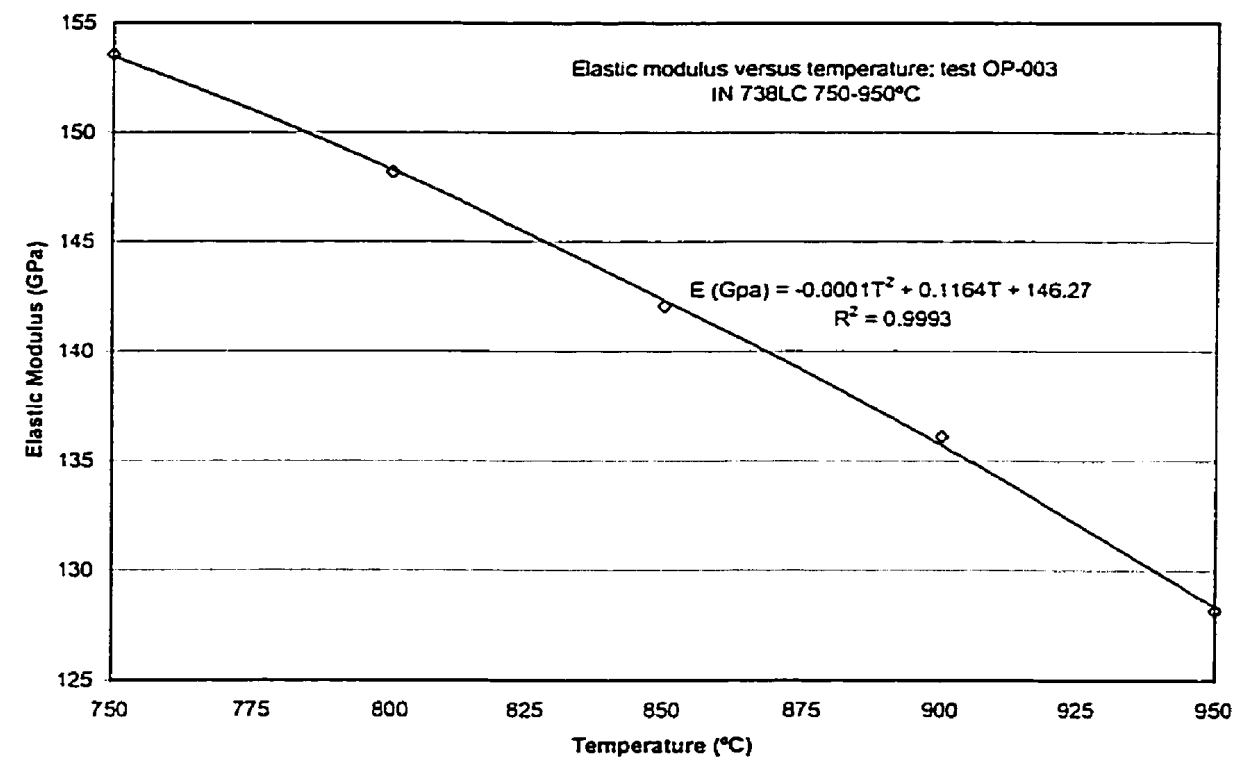

Figure 4-20 - Typical variation in elastic modulus, for the IN738LC material used in the present study, with temperature determined before starting a TMF test.

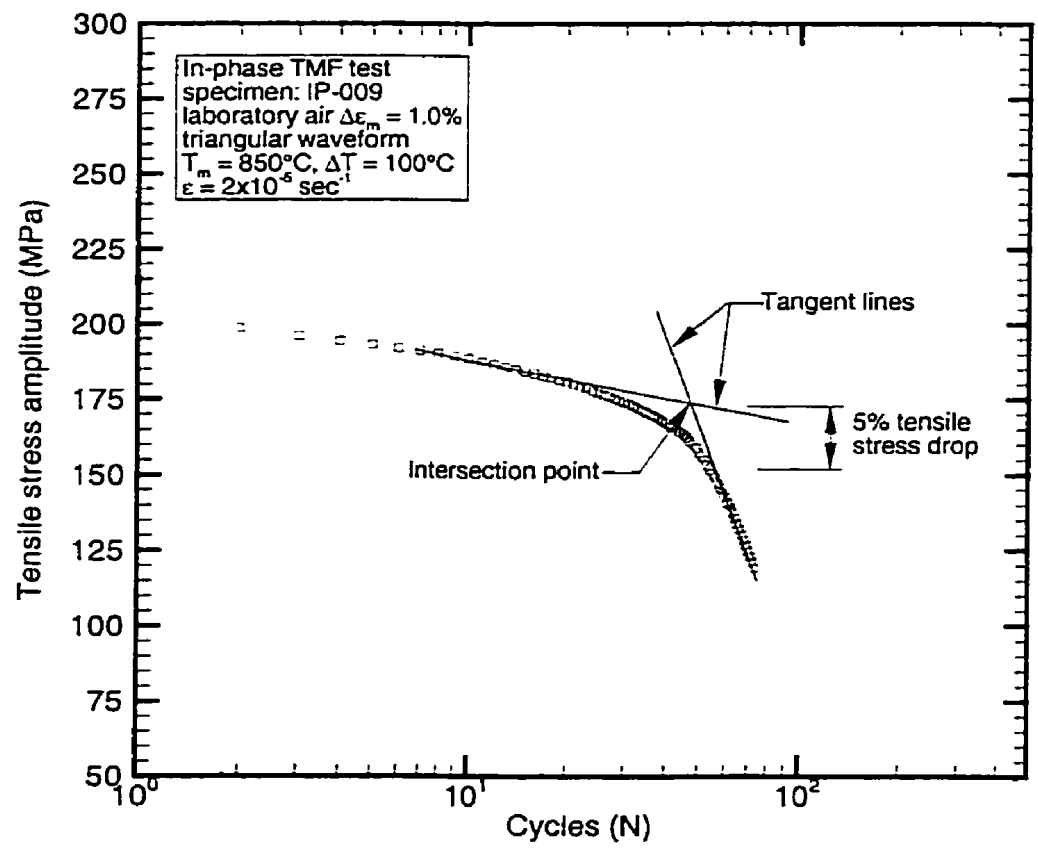

Figure 4-21 - Definition of 5\% tensile stress amplitude change based on the intersection point of a line tangent to the uniform cyclic response region, and a line tangent to rapid load drop region of the cyclic stress response. 


\section{IT-LCF and TMF Commissioning Tests}

\subsection{Introduction}

To demonstrate the capability of the TMF testing facility and the soundness of the experimental approach used for the present work, a series of commissioning tests were conducted. The commissioning tests, material, specimens, and test results are described in the following sections.

\subsection{Experimental}

The cyclic life of IN738LC superalloy was investigated under IP and OP-TMF cycling, with a temperature range of $750^{\circ} \mathrm{C}$ to $950^{\circ} \mathrm{C}$, and IT-LCF cycling at $950^{\circ} \mathrm{C}$. The tests were fully reversed $\left(R_{\varepsilon}=-1\right)$, and conducted at different constant mechanical strain ranges varying between 0.4 to $1.0 \%$. A constant mechanical strain rate of $2 \times 10^{-5} \mathrm{sec}^{-1}$ was used for all tests. The mechanical strain rate in this investigation was fixed by the lowest mechanical strain range, and the maximum temperature cycling rate of $1.0^{\circ} \mathrm{C} \mathrm{sec}$ (see Chapter 4). The temperature rate was varied between 0.4 to $1^{\circ} \mathrm{C} \mathrm{sec}^{-1}$ in TMF tests since it was coupled to the mechanical strain range. Specimen failure was defined as a $5 \%$ drop in cyclic peak tensile stress amplitude (see Chapter 4 for definition of failure). All tests were repeated two or three times since a considerable amount of scatter was observed in low strain range tests. 


\subsubsection{Material}

The material selected for the TMF and LCF commissioning tests was the investment cast nickel-base superalloy IN738LC. The primary applications for this material are in blades and vanes in gas turbine engines requiring good hot corrosion resistance. The nominal chemical composition of the alloy IN738LC is given in Table 5-1.

The IN738LC material was received in the form of $200 \times 125 \times 19 \mathrm{~mm}$ investment cast plates from Howmet Corporation. Since IN738LC is subject to the formation of casting microporosity during solidification, the cast plates were hot isostatically pressed (HIP'ed) for 4 hours at $1205^{\circ} \mathrm{C}$ at a pressure of $1034 \mathrm{Bar}$ by the foundry. Porosity of the cast plates was specified to not exceed $0.25 \%$ of a $100 \mathrm{x}$ field and a maximum pore size of $0.05 \mathrm{~mm}$. Casting porosity was not observed in several sections of material cut from the cast plates.

Prior to heat treating the material, the cast plates were sectioned into $19 \times 19 \times 125 \mathrm{~mm}$ specimen blanks by a wet abrasive cut-off saw. The bars were heat-treated in a radiation

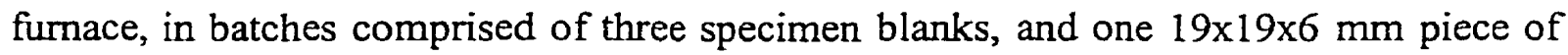
the same alloy to serve as a 'quality control' sample. The standard solution and precipitation hardening cycle for $\mathbb{N} 738 \mathrm{LC}$ was used in the present investigation. The standard heat treatment is: solution treat $2 \mathrm{~h}$ at $1120^{\circ} \mathrm{C}$, air cool to room temperature, precipitation harden $24 \mathrm{~h}$ at $845^{\circ} \mathrm{C}$, air cool to room temperature.

Following the heat treatment, selected bars were sectioned, mounted, and progressively polished from 320,400 , and 600 grit paper, followed by $9 \mu \mathrm{m}, 3 \mu \mathrm{m}$, and $1 \mu \mathrm{m}$ diamond 
paste. To reveal the $\gamma^{\prime}$ precipitate morphology, the samples were electrolytically etched in a $12 \mathrm{ml} \mathrm{H}_{3} \mathrm{PO}_{4}+40 \mathrm{ml} \mathrm{HNO}_{3}+48 \mathrm{ml} \mathrm{H}_{2} \mathrm{SO}_{4}$ solution with an applied potential of 6 VDC for 10 seconds. To reveal the grain boundary and carbide morphology, the samples were electrolytically etched in a $10 \% \mathrm{HCl}+90 \%$ methanol solution with an applied potential of 4 VDC for 60 seconds. The microstructures were characterized by optical and scanning electron microscopy (SEM).. The standard heat treatment produces a bimodal $\gamma^{\prime}$ distribution consisting of approximately $0.5 \mu \mathrm{m}$ cuboidal primary, and $0.08 \mu \mathrm{m}$ spherical secondary $\gamma^{\prime}$ of approximately $43 \%$ volume fraction (see Figure 5-1). Figure 5-2, shows a transverse and longitudinal section through a specimen blank, etched to reveal the grain boundary morphology and the grain size in the cast plate. Script $\mathrm{MC}$ and discrete $\mathrm{M}_{23} \mathrm{C}_{6}$ carbides are present at grain boundaries (see Figure 5-3). Script MC carbides were also observed in interdendritic regions.

\subsubsection{Test Specimens}

Two different specimen geometries were used for the commissioning tests in the present study. The two specimen geometries were based on guidelines given by the ASTM E606$92 \mathrm{LCF}^{82}$ testing standard. Solid specimens were used for both IT-LCF and TMF tests. For the IT-LCF tests, a standard geornetry was adopted as illustrated in Figure 5-4. For the TMF tests, a specimen with an extended gage section was utilized, since the extended section simplified the relationship between heating coil adjustments (adjustments to coil coupling and pitch) and changes to dynamic temperature distribution within the gage section (see Figure 5-5). 
Test specimens were machined using the guidelines specified in ASTM E606-92 Appendix $X 3^{82}$. After machining, all specimens were progressively polished axially with 320,400 and 600 grit $\mathrm{SiC}$ paper to remove all circumferential machining marks. The quality of surface finish was verified by viewing the polished specimens under an optical microscope at $25 \mathrm{x}$. Before testing, specimens were cleaned ultrasonically in an acetone bath for 10 minutes and then hot air dried.

\subsubsection{Post Test Analysis}

Following testing, specimens were pulled apart at room temperature. In order to identify the cracking process, longitudinal sections perpendicular to the fracture surface were removed from tested specimens, and mounted in epoxy for metallographic analysis. Some sections were etched to reveal the grain boundaries while others were etched to reveal the $\gamma^{\prime}$ morphology using the procedures given in Section 5.2.1. Crack initiation sites and propagation paths were examined by optical and scanning electron microscopy. The density of secondary cracks, crack initiation and propagation modes, and changes to $\gamma$ ' morphology after testing were assessed from the longitudinal sections.

\subsection{Results and Discussion}

\subsubsection{Stress-Strain Behavior and Fatigue Life}

The experimental results from the TMF facility commissioning tests are summarized in Tables 2 to 4 . All test results are reported in these tables, with the exception of specimens that failed outside of the gage length. Figure 5-6, shows representative stress-strain 
hysteresis loops typical of IP and OP-TMF, and IT-LCF tests at the same mechanical strain range of $1 \%$. The IT-LCF hysteresis loops were symmetrical about the abscissa and had negligible mean stress. The IP and OP-TMF hysteresis loops were asymmetric; however, they are similar in shape and appear rotated $180^{\circ}$ about the origin with respect to one another. For OP-TMF cycling a tensile mean stress was observed, whereas IP TMF cycling results in a compressive mean stress. The cyclic stress-strain curves ( $\sigma$ versus $\Delta \varepsilon_{\text {mech }}$ ) at half-life for IT-LCF, OP-TMF and IP-TMF are presented in Figure 5-7. The tensile stress amplitude for IP-TMF tests and compressive stress amplitude for OPTMF tests are clearly similar. OP-TMF tests exhibit higher tensile stress amplitudes than IT-LCF and IP-TMF loading conditions. The higher tensile stress amplitude exhibited by OP-TMF tests is due to the fact that tensile loading occurs at a lower temperature than ITLCF or IP-TMF loading conditions. The typical cyclic stress response for IT-LCF, OPTMF and IP-TMF tests is illustrated in Figure 5-8. The tensile cyclic flow stress softened during IP-TMF and IT-LCF loading, whereas cyclic hardening is observed during OPTMF loading. The compressive cyclic stress response during OP-TMF loading is similar to the tensile response during IP-TMF loading.

Experimental results for IT-LCF, OP-TMF and IP-TMF tests are presented as mechanical strain-life curves $\left(\Delta \varepsilon_{\text {mech }}\right.$ versus $\left.N_{95}\right)$ in Figure 5-9. Out-of-phase TMF loading produce longer lives than IP-TMF and IT-LCF loading (as high as 3.3 times) at high mechanical strain ranges $\left(\Delta \varepsilon_{\text {mech }}=1.0 \%\right)$. In-phase TMF and IT-LCF loading results in a longer fatigue lives than OP-TMF (as high as 1.6 times) at low mechanical strain ranges $\left(\Delta \varepsilon_{\text {mech }}\right.$ $=0.45 \%$ ). For all mechanical strain ranges considered in this investigation, IP-TMF 
exhibits a longer fatigue life than IT-LCF loading. Therefore, the IT-LCF tests provide a conservative estimate of IP-TMF life, for the mechanical strain ranges considered in this investigation. A distinct crossover of the OP-TMF and IP-TMF mechanical strain life curves is observed at an intermediate mechanical strain range (approximately $0.6 \%$ ). A number of other investigators have reported a similar crossover of the IP and OP-TMF strain life curves in nickel-base superalloys ${ }^{36,42,47}$. Boismer and Sehitioglu ${ }^{42}$ suggest that one possible explanation for the crossover of the IP-TMF and OP-TMF strain life curves lies in the relative contribution of fatigue, oxidation, and creep damage.

The mechanical strain-life curve does not differentiate between the elastic and inelastic components of strain. It is essential to consider the inelastic strain component since lowcycle fatigue damage, which is due to inelastic strain, is the dominant damage mechanism when other damage mechanisms (creep or oxidation) are absent. Typically, low-cycle fatigue results are presented as a plot of inelastic strain range $\left(\Delta \varepsilon_{\text {in }}\right)$ versus life $(N)$. In order to observe the role of inelastic strain on fatigue life, inelastic strain range $\left(\Delta \varepsilon_{\text {in }}\right)$ versus life $\left(\mathrm{N}_{95}\right)$ was plotted for IT-LCF, IP-TMF and OP-TMF tests (see Figure 5-10). Isothermal-LCF tests exhibit longer lives than IP-TMF tests (as high as 1.5 times) when inelastic strain range is considered. The crossover of the IP-TMF and OP-TMF strain life curves is more obvious when plotted as inelastic strain versus life. Out-of-phase TMF life is distinctly higher than IP-TMF and IT-LCF tests (as high as 2.3 times) at high inelastic strain ranges $\left(\Delta \varepsilon_{\text {in }}=0.57 \%\right.$ ), and considerably shorter (as high as 3 times) at low inelastic strain ranges $\left(\Delta \varepsilon_{\text {in }}=0.13 \%\right)$. For the remainder of this discussion, the results presented in 
Figure 5-9 will be related to metallographic observations, and the cyclic stress-strain response for IT-LCF and TMF loading conditions.

In order to differentiate between loading conditions (TMF versus IT-LCF), and resulting inelastic strain response, inelastic strain range $\left(\Delta \varepsilon_{\text {in }}\right)$ was plotted versus mechanical strain range $\left(\Delta \varepsilon_{\text {mech }}\right)$ for IT-LCF, OP-TMF and IP-TMF tests (see Figure 5-11). The inelastic strain range for IP and OP-TMF tests are clearly lower than IT-LCF tests, for all mechanical strain ranges considered in this investigation. One possible explanation the different inelastic strain ranges between TMF and IT-LCF tests lies in the effect of temperature on elastic modulus and flow stress during TMF loading. Perhaps the most significant result that can be obtained from this plot is that IP and OP-TMF tests exhibit a similar inelastic strain response when compared at the same mechanical strain range. If one considers this observation from the perspective of "pure" fatigue damage the cyclic life of IP and OP-TMF tests should be similar. On the contrary, Figure 5-10 clearly shows that the cyclic lives of IP and OP-TMF tests are radically different at high and low strain ranges. These observations suggest that the dominant damage mechanism(s) for IP and OP-TMF tests are different.

\subsubsection{Microstructural Observations}

Longitudinal sections from TMF and IT-LCF test specimens were examined by optical and scanning electron microscopy to determine the fatigue initiation and propagation modes, and evaluate microstructural changes for each loading condition. No significant variation in crack initiation and propagation modes was observed in IT-LCF, IP-TMF and 
OP-TMF specimens. Crack initiation was predominantly intergranular and associated with preferentially oxidized grain boundaries that intersect with the specimen surface (see Figure 5-12 A to C). Fatigue crack propagation was predominantly intergranular; however, it was also observed that intergranularly initiated cracks switch to transgranular mode when grain boundaries are not located perpendicular to the loading axis (see Figure 5-13 A to C). Extensive cracking of internal grain boundaries was observed in all IPTMF specimens (see Figure 5-14). No internal grain boundary cracks were observed in OP-TMF or IT-LCF specimens. These observations suggest that creep is the dominant damage mechanism in IP-TMF tests. Sehitoglu and Boismer $^{48}$ reached a similar conclusion for IP-TMF tests on the nickel-base superalloy Mar-M247. At high strain ranges, the lower cyclic life of IP-TMF tests, when compared to OP-TMF tests, is probably due to the contribution of creep damage to failure during IP-TMF loading. The lower inelastic strain-life exhibited by IP-TMF tests, when compared to IT-LCF tests, is also probably due to the contribution of creep damage during IP-TMF loading.

Metallographic examination of OP-TMF, IP-TMF and IT-LCF specimens revealed another significant difference between OP-TMF and the other loading conditions. The density of surface cracks observed on IP-TMF and IT-LCF specimens was considerably higher than OP-TMF specimens. This suggests that fatigue crack growth is a more predominant process under OP-TMF loading. Chen et al. have come to a similar conclusion for OP-TMF tests on $\mathbb{N} 738 \mathrm{LC}^{41}$. Henderson et al. ${ }^{113}$ have pointed out that different crack propagation paths may cause a difference in crack growth rates. The crack propagation paths were similar for the TMF and IT-LCF tests in this investigation. Chen 
et al. $^{41}$ have determined that crack initiation in $\mathrm{N} 738 \mathrm{LC}$ is dependent on the oxidation of grain boundaries that intersect the specimen surface. Since TMF tests are exposed to the maximum test temperature for relatively short periods, when compared to IT-LCF tests, it is expected that less oxide penetration will occur along grain boundaries during TMF tests than IT-LCF tests of the same duration. At high strain ranges, the longer life exhibited by OP-TMF tests, when compared with IT-LCF tests, was probably due to a larger portion of cyclic life spent in initiating a fatigue cracks than in IT-LCF tests. On the other hand, tensile loading occurs at low temperature $\left(750^{\circ} \mathrm{C}\right)$ in OP-TMF tests where the fatigue crack propagation rate has been measured to be higher than at a higher temperature $\left(850^{\circ} \mathrm{C}\right)^{114}$. Therefore, at low strain ranges the shorter life exhibited by OPTMF tests, when compared to IP-TMF and IT-LCF tests, is probably due to higher fatigue crack propagation rates.

The specimens were also examined for changes to the $\gamma^{\prime}$ precipitate morphology. Figure 5-15 A to C, shows representative photomicrographs of the $\gamma^{\prime}$ precipitate morphology after IT-LCF, IP-TMF and OP-TMF testing. When these photomicrographs are compared to the $\gamma^{\prime}$ morphology before testing (see Figure 5-1) it appears that dissolution the secondary $\gamma^{\prime}$ has occurred. Further investigation is necessary to determine whether dissolution of secondary $\gamma^{\prime}$ has had any significant effect on the morphology of the primary $\gamma^{\prime}$ precipitates. Antolovich et al. ${ }^{28}$ have associated cyclic softening observed during LCF tests on the Ni-base superalloy Rene 80, with rapid coarsening of large $\gamma^{\prime}$ precipitates, development of interfacial dislocations on the $\gamma^{\prime}$, and dissolution of the small $\gamma^{\prime}$ precipitates. Perhaps, the observed changes to $\gamma^{\prime}$ morphology in this investigation may 
partially explain the cyclic stress response observed in TMF and IT-LCF- tests (see Figure 5-8). Further metallographic examination using transmission electron microscopy (TEM) is necessary to investigate the dislocation mechanisms operative under IP-TMF, OPTMF, and IT-LCF loading conditions in the material considered in this investigation (IN738LC). 


\begin{tabular}{|c|c|c|c|c|c|c|c|c|c|c|l|l|}
\hline Element & $\mathrm{Ni}$ & $\mathrm{Cr}$ & $\mathrm{Co}$ & $\mathrm{Al}$ & $\mathrm{Ti}$ & $\mathrm{W}$ & $\mathrm{B}$ & $\mathrm{Ta}$ & $\mathrm{Mo}$ & $\mathrm{Zr}$ & $\mathrm{Nb}$ & $\mathrm{C}$ \\
\hline$(\%)$ & Bal. & 16 & 8.5 & 3.5 & 3.5 & 2.6 & 0.01 & 1.8 & 1.8 & 0.04 & 0.9 & $0.1-0.15$ \\
\hline
\end{tabular}

Table 5-1 - Nominal composition of IN 738LC in wt\%.

\begin{tabular}{|c|c|c|c|c|c|c|c|c|}
\hline Test ID & $\begin{array}{c}\Delta \varepsilon_{\text {mech }} \\
\text { applied (\%) }\end{array}$ & $\mathrm{N}_{95}$ & $\begin{array}{c}\sigma_{\max } \\
\mathrm{MPa}\end{array}$ & $\begin{array}{c}\sigma_{\min } \\
\mathrm{MPa}\end{array}$ & $\begin{array}{c}\sigma_{\text {mean }} \\
\mathrm{MPa}\end{array}$ & $\begin{array}{l}\Delta \varepsilon_{\text {mech }} \\
(\%)\end{array}$ & $\begin{array}{l}\Delta \varepsilon_{\text {in }} \\
(\%)\end{array}$ & $\begin{array}{l}\Delta \varepsilon_{\mathrm{e}} \\
(\%)\end{array}$ \\
\hline IT-003 & 1.0 & 42 & 178.2 & -168.9 & 9.3 & 0.999 & 0.670 & 0.329 \\
\hline IT-005 & 1.0 & 38 & 177.6 & -168.4 & 9.2 & 0.999 & 0.684 & 0.315 \\
\hline IT-001 & 0.8 & 77 & 174.0 & -162.5 & 11.5 & 0.799 & 0.492 & 0.307 \\
\hline IT-006 & 0.8 & 70 & 175.8 & -164.5 & 11.3 & 0.799 & 0.544 & 0.255 \\
\hline IT-002 & 0.6 & 335 & 157.6 & -147.7 & 9.9 & 0.599 & 0.322 & 0.277 \\
\hline IT-004 & 0.6 & 135 & 154.0 & -145.0 & 9.0 & 0.599 & 0.335 & 0.264 \\
\hline IT-007 & 0.6 & 180 & 163.9 & -153.2 & 10.7 & 0.599 & 0.306 & 0.294 \\
\hline IT-010 & 0.45 & 220 & 159.6 & -147.6 & 12.0 & 0.449 & 0.224 & 0.225 \\
\hline IT-011 & 0.45 & 520 & 148.4 & -139.0 & 9.4 & 0.448 & 0.186 & 0.262 \\
\hline IT-012 & 0.45 & 800 & 148.4 & -139.0 & 9.4 & 0.449 & 0.178 & 0.271 \\
\hline IT-008 & 0.4 & 1175 & 140.9 & -131.4 & 9.5 & 0.396 & 0.157 & 0.239 \\
\hline
\end{tabular}

Table 5-2-IT-LCF testing results at $950^{\circ} \mathrm{C}$.

\begin{tabular}{|c|c|c|c|c|c|c|c|c|}
\hline Test ID & $\begin{array}{c}\Delta \varepsilon_{\text {mech }} \\
\text { applied (\%) }\end{array}$ & $\mathrm{N}_{95}$ & $\begin{array}{c}\sigma_{\max } \\
\mathrm{MPa}\end{array}$ & $\begin{array}{c}\sigma_{\min } \\
\mathrm{MPa}\end{array}$ & $\begin{array}{c}\sigma_{\text {mean }} \\
\mathrm{MPa}\end{array}$ & $\begin{array}{c}\Delta \varepsilon_{\operatorname{mech}} \\
(\%)\end{array}$ & $\begin{array}{c}\Delta \varepsilon_{\text {in }} \\
(\%)\end{array}$ & $\begin{array}{c}\Delta \varepsilon_{\mathrm{e}} \\
(\%)\end{array}$ \\
\hline OP-004 & 1.0 & 133 & 514.1 & -182.4 & 331.7 & 0.999 & 0.565 & 0.434 \\
\hline OP-005 & 1.0 & 95 & 482.9 & -176.8 & 306.1 & 0.999 & 0.583 & 0.416 \\
\hline OP-003 & 0.8 & 200 & 449.3 & -132.7 & 316.6 & 0.798 & 0.341 & 0.456 \\
\hline OP-006 & 0.8 & 230 & 464.3 & -138.2 & 326.1 & 0.798 & 0.345 & 0.453 \\
\hline OP-001 & 0.6 & 345 & 457.1 & -125.6 & 331.5 & 0.599 & 0.214 & 0.385 \\
\hline OP-002 & 0.6 & 175 & 487.5 & -137.8 & 349.7 & 0.598 & 0.217 & 0.381 \\
\hline OP-008 & 0.45 & 470 & 424.5 & -115.0 & 309.5 & 0.448 & 0.103 & 0.345 \\
\hline OP-009 & 0.45 & 317 & 447.9 & -119.5 & 328.4 & 0.450 & 0.130 & 0.320 \\
\hline OP-010 & 0.45 & 750 & 360.1 & -96.9 & 263.2 & 0.449 & 0.053 & 0.396 \\
\hline
\end{tabular}

Table 5-3-OP-TMF testing results. 


\begin{tabular}{|l|c|c|c|c|c|c|c|c|}
\hline Test ID & $\begin{array}{c}\Delta \varepsilon_{\text {mech }} \\
\text { applied (\%) }\end{array}$ & $N_{95}$ & $\begin{array}{c}\sigma_{\max } \\
\mathrm{MPa}\end{array}$ & $\begin{array}{c}\sigma_{\min } \\
\mathrm{MPa}\end{array}$ & $\begin{array}{c}\sigma_{\text {mean }} \\
\mathrm{MPa}\end{array}$ & $\begin{array}{c}\Delta \varepsilon_{\text {mech }} \\
(\%)\end{array}$ & $\begin{array}{c}\Delta \varepsilon_{\text {in }} \\
(\%)\end{array}$ & $\begin{array}{c}\Delta \varepsilon_{\mathrm{e}} \\
(\%)\end{array}$ \\
\hline IP-008 & 1.0 & 55 & 180.3 & -535.3 & -355.0 & 0.998 & 0.538 & 0.460 \\
\hline $\mathrm{IP}-009$ & 1.0 & 52 & 154.8 & -496.0 & -341.2 & 0.997 & 0.532 & 0.465 \\
\hline $\mathrm{IP}-001$ & 0.8 & 170 & 141.7 & -488.1 & -346.4 & 0.790 & 0.385 & 0.406 \\
\hline $\mathrm{IP}-002$ & 0.8 & 117 & 147.1 & -480.7 & -333.6 & 0.799 & 0.359 & 0.440 \\
\hline $\mathrm{IP}-007$ & 0.6 & 220 & 132.9 & -502.6 & -369.7 & 0.598 & 0.228 & 0.370 \\
\hline IP-010 & 0.6 & 498 & 108.5 & -397.9 & -289.4 & 0.599 & 0.154 & 0.445 \\
\hline IP-003 & 0.6 & 155 & 125.2 & -469.2 & -344.0 & 0.599 & 0.180 & 0.419 \\
\hline IP-005 & 0.45 & 975 & 112.0 & -400.6 & -288.6 & 0.449 & 0.178 & 0.271 \\
\hline
\end{tabular}

Table 5-4 - IP-TMF testing results. 


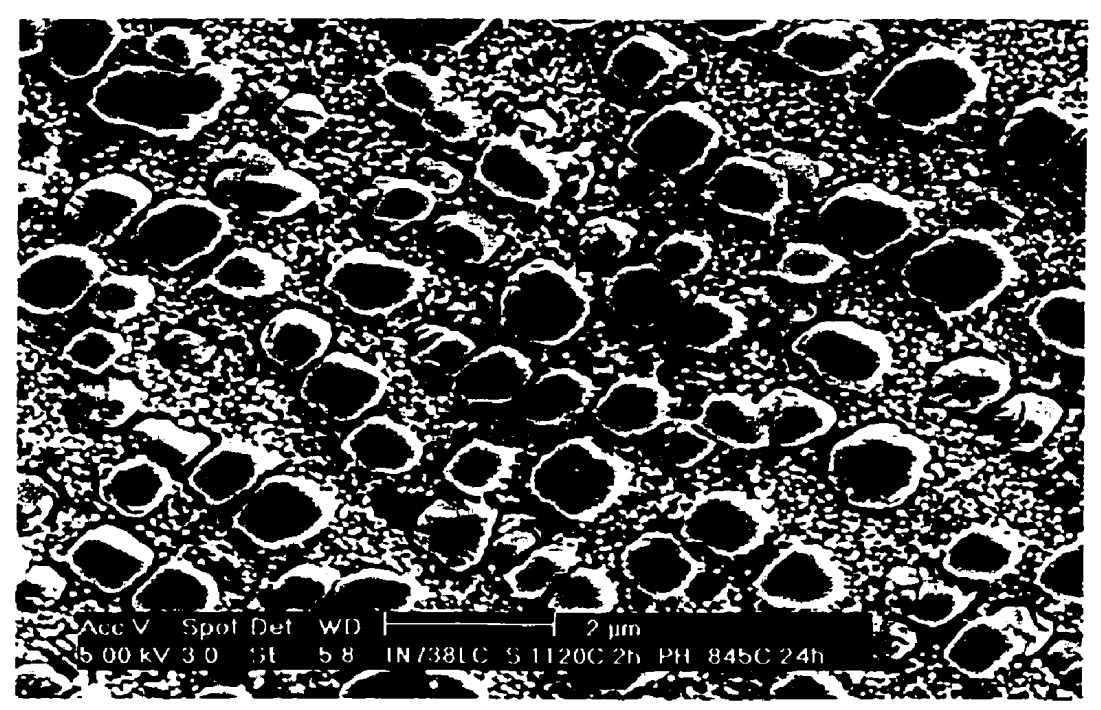

Figure 5-1 - SEM micrograph illustrating bimodal $\gamma^{\prime}$ distribution in standard heat-treated IN738LC.
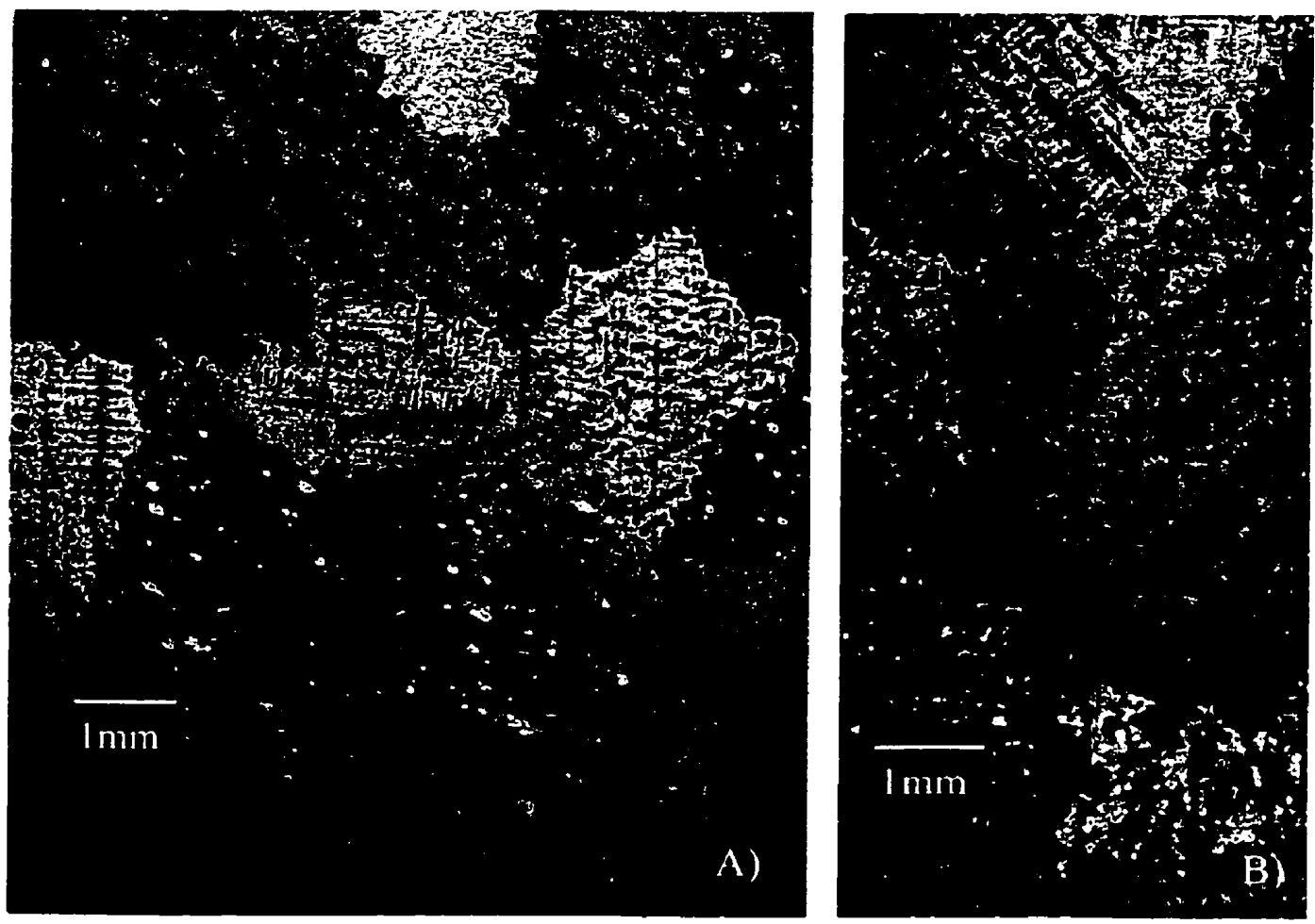

Figure 5-2 - (A) Longitudinal and (B) transverse section through specimen blank revealing the grain size of the investment cast $\mathrm{N} 738 \mathrm{LC}$ used in this investigation. 

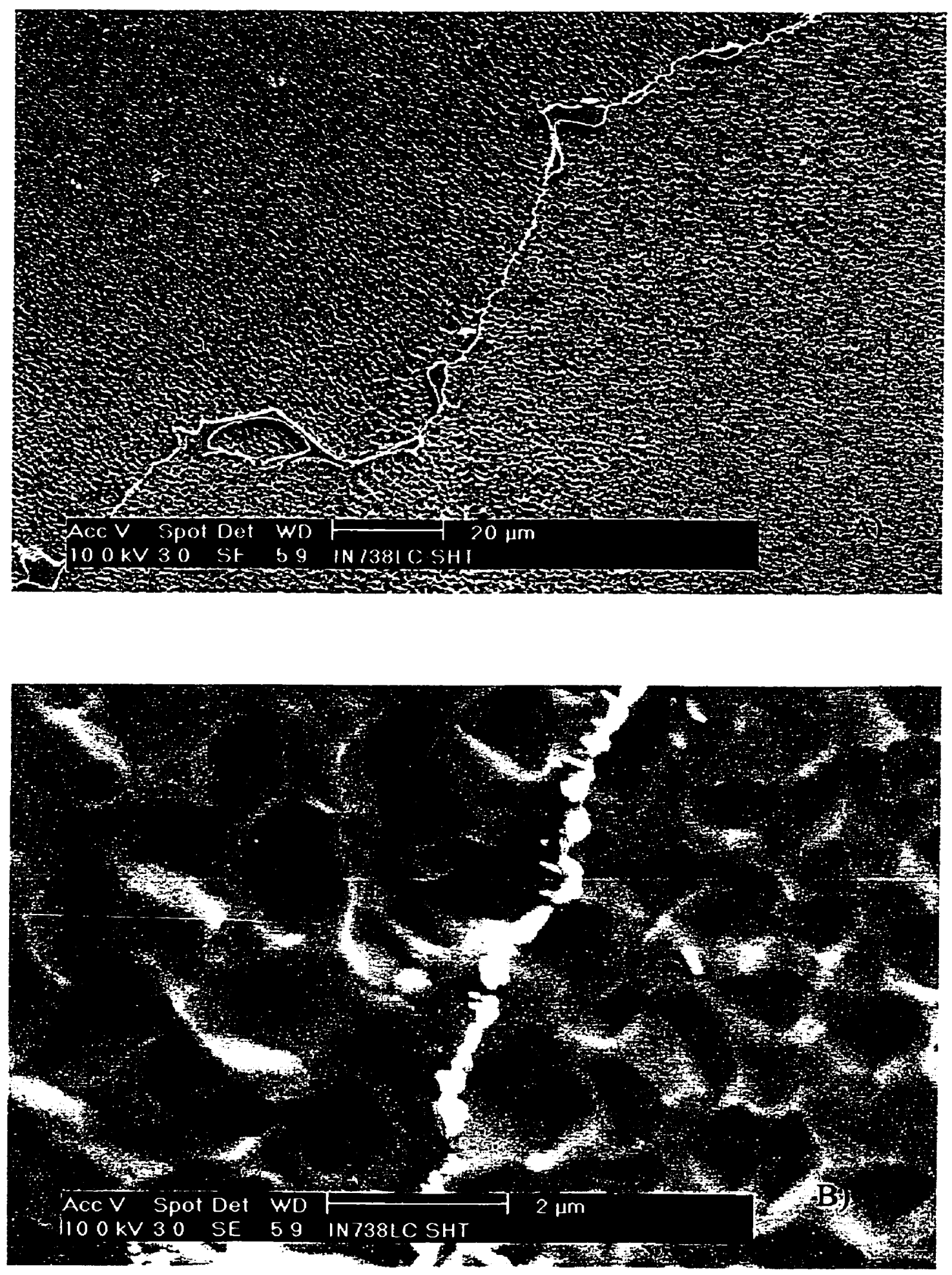

Figure 5-3 - (A) SEM micrograph illustrating script MC carbides and grain boundary morphology. (B) A higher magnification view of a grain boundary showing discrete $\mathrm{M}_{23} \mathrm{C}_{6}$ carbides. 


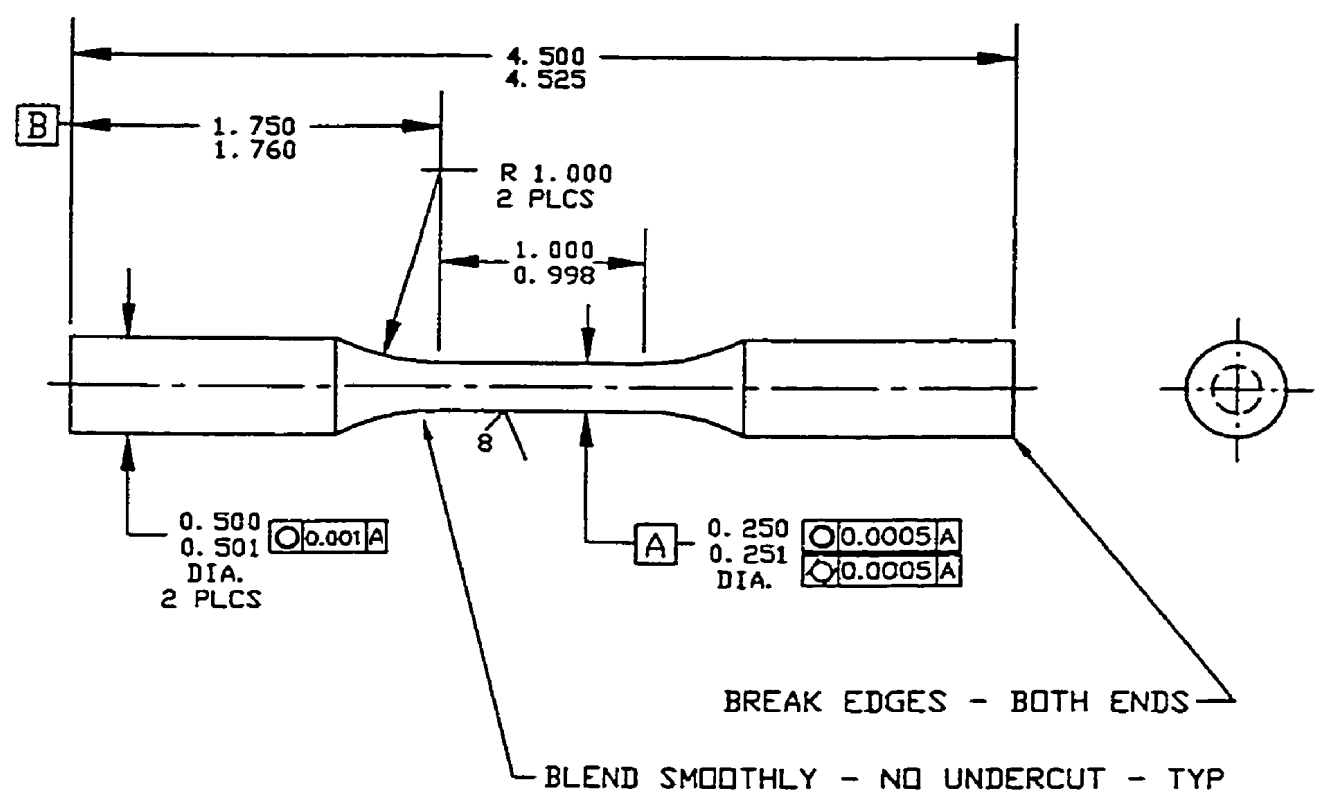

Figure 5-4 - Specimen design for IT-LCF testing.

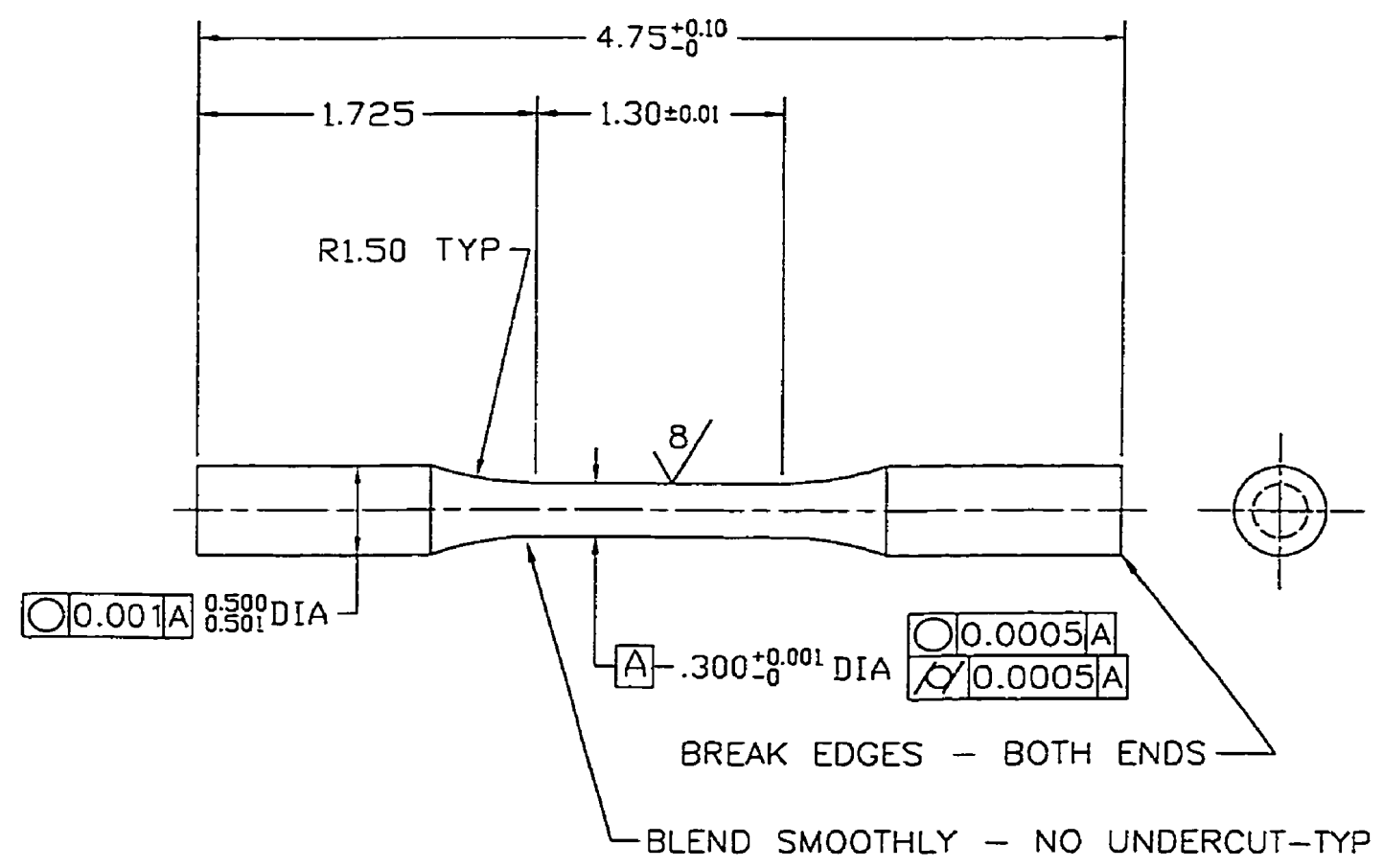

Figure 5-5 - Specimen design for TMF testing. 


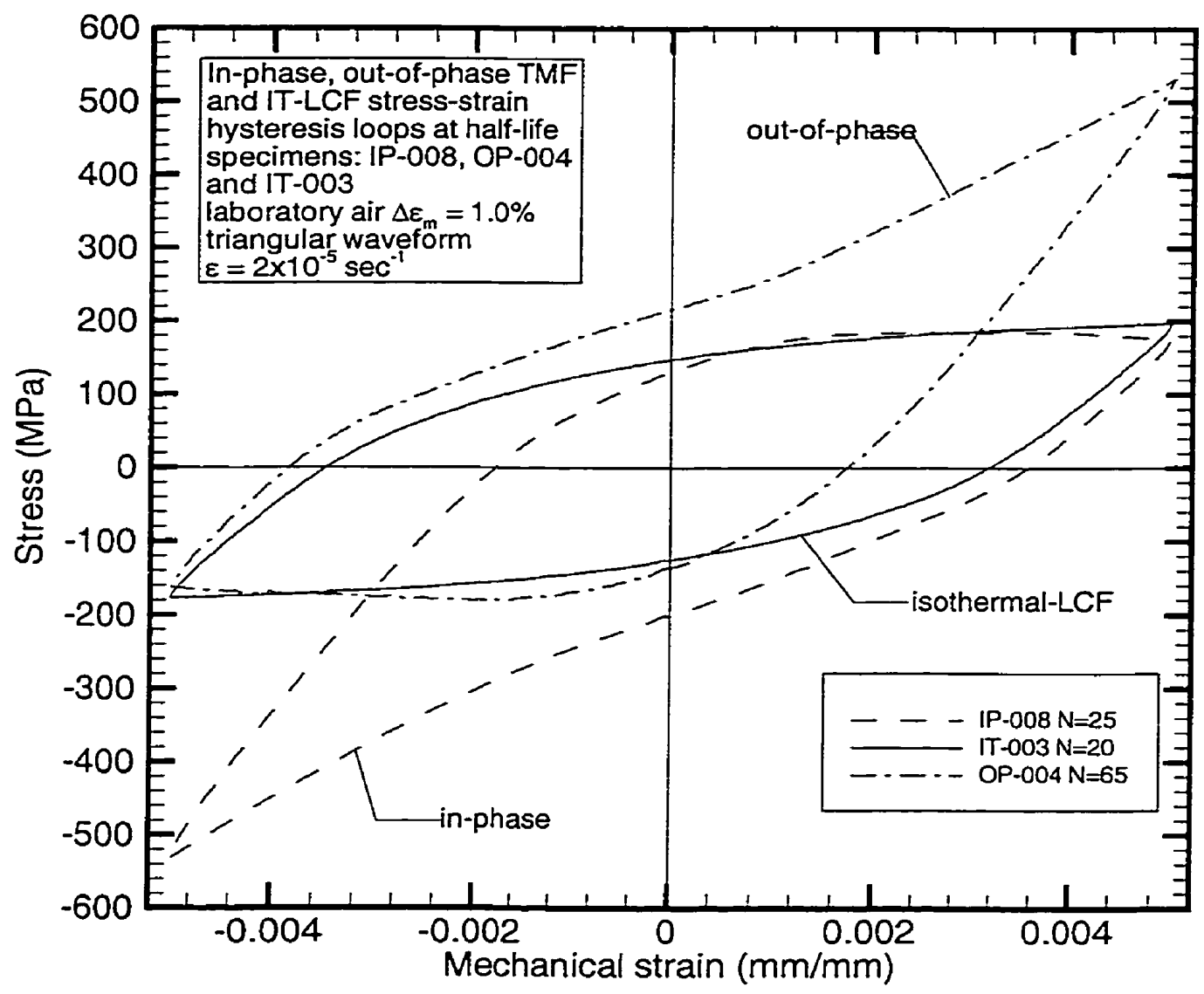

Figure 5-6 - Experimental stress-strain hysteresis loops representative of in-phase TMF, out-of-phase TMF, and isothermal-LCF tests at $1 \%$ mechanical strain range. 


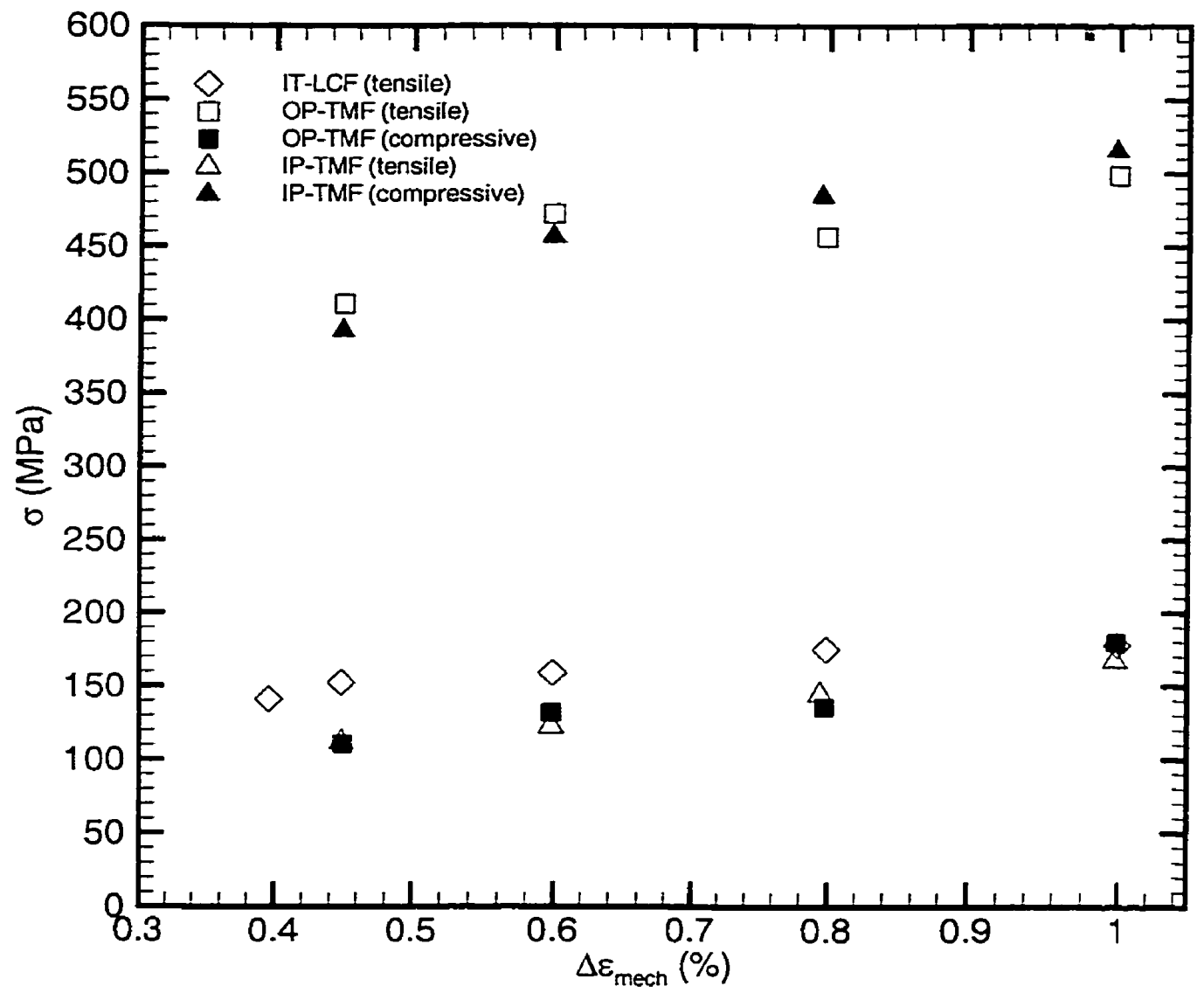

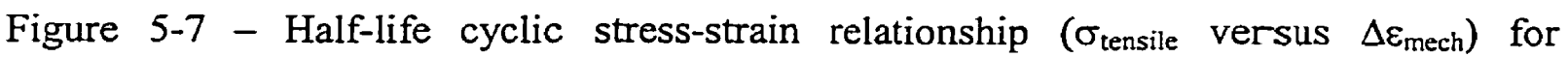
isothermal-LCF, out-of-phase TMF and in-phase TMF tests. Note: the points shown on this figure are based on the average stress amplitude for two or three tests:. 


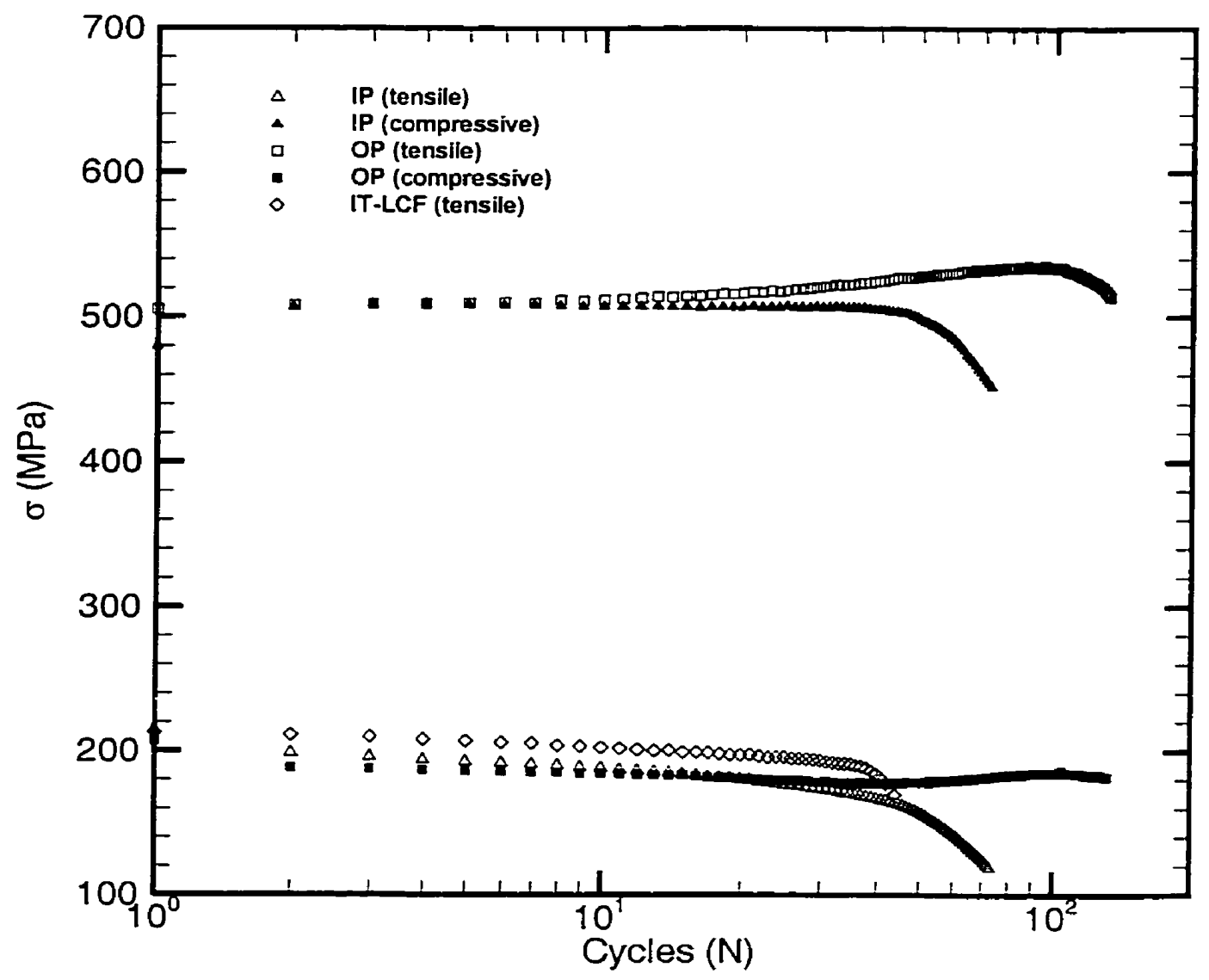

Figure 5-8 - Typical cyclic stress response for isothermal-LCF, in-phase TMF and outof-phase TMF tests at $1 \%$ mechanical strain range. 


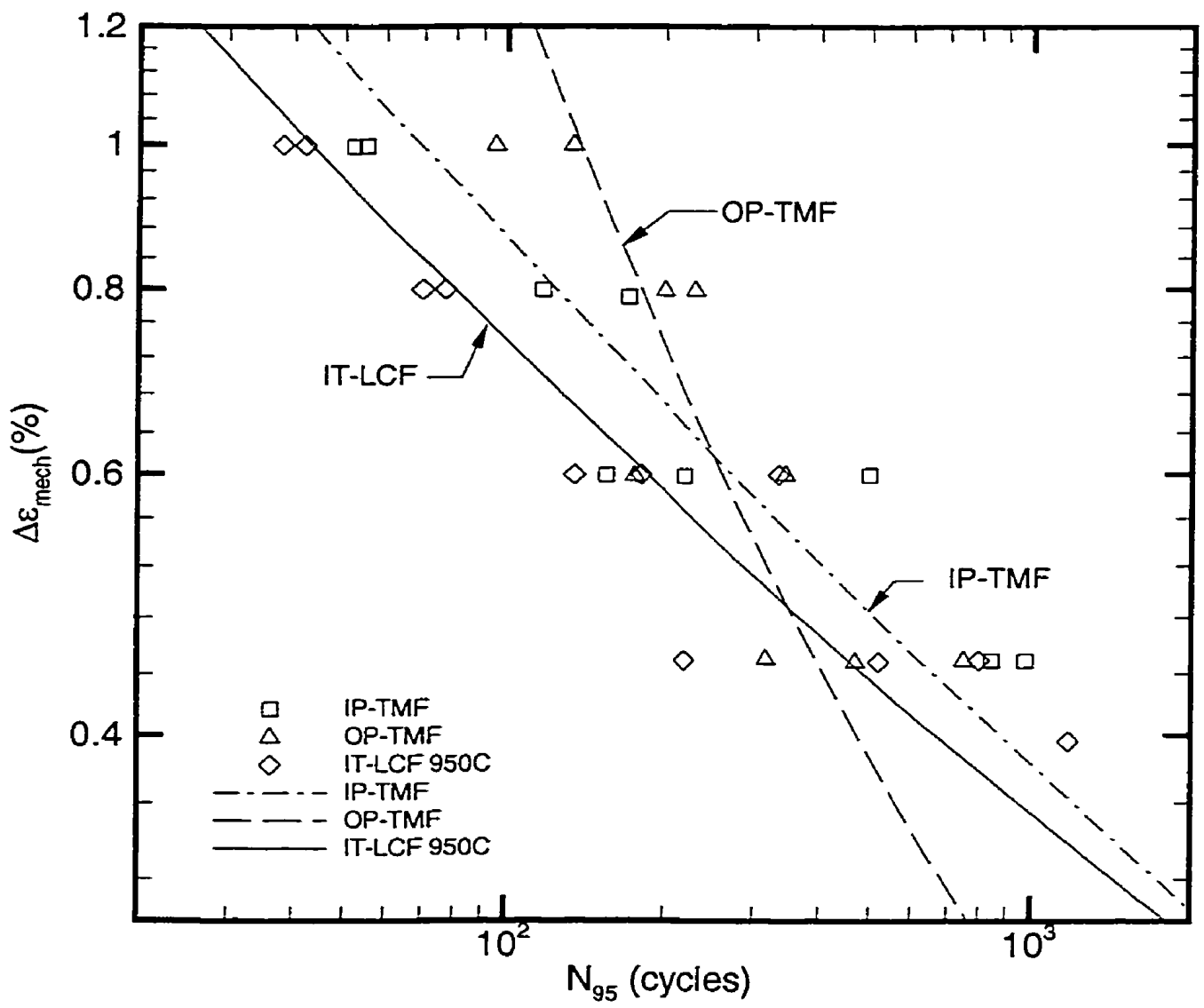

Figure 5-9 - Mechanical strain range versus life to 5\% tensile stress amplitude drop for isothermal-LCF, in-phase TMF and out-of-phase TMF tests. 


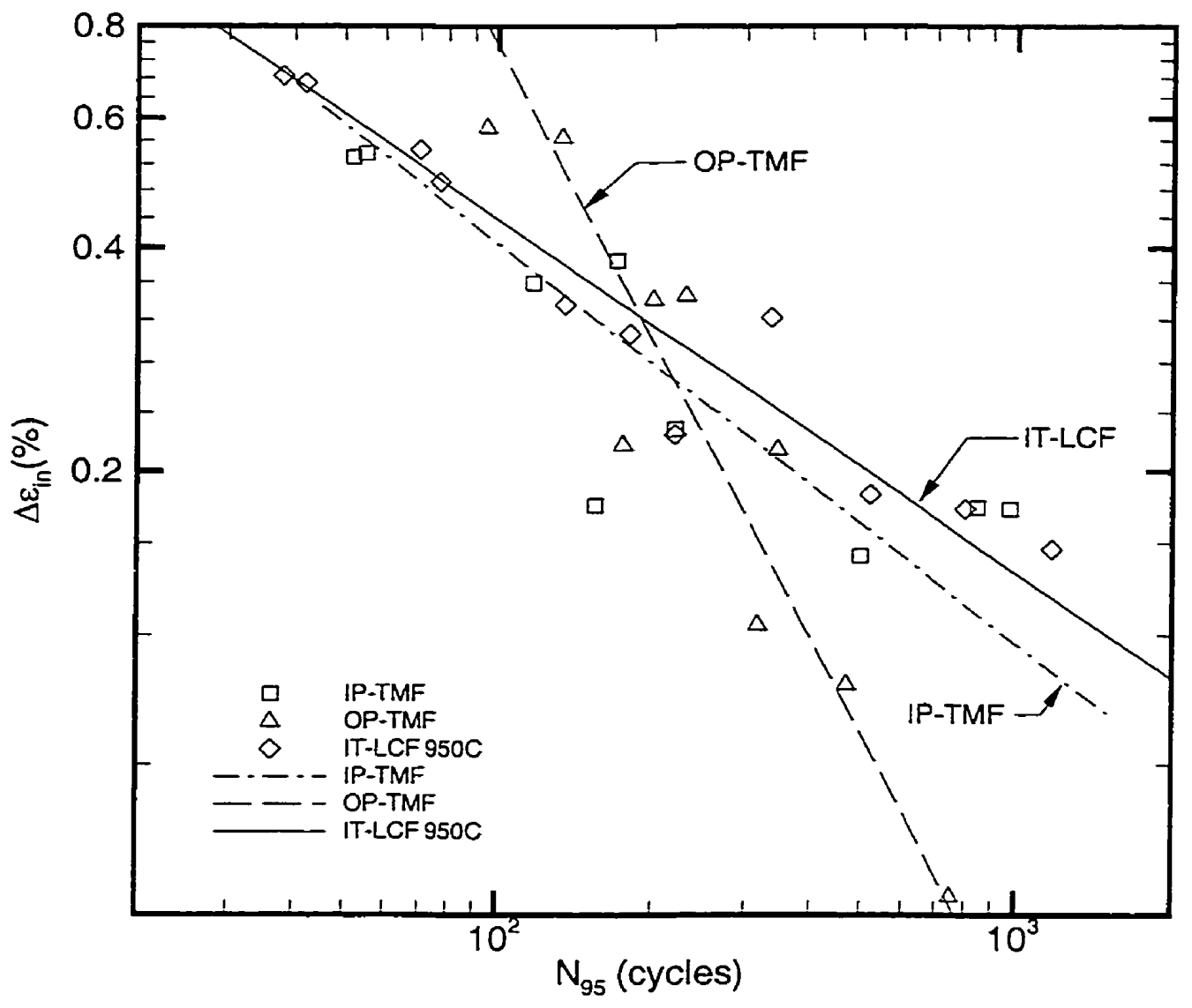

Figure 5-10 - Inelastic strain range versus life to 5\% tensile stress amplitude drop for inphase TMF, out-of-phase TMF, and isothermal-LCF tests. 


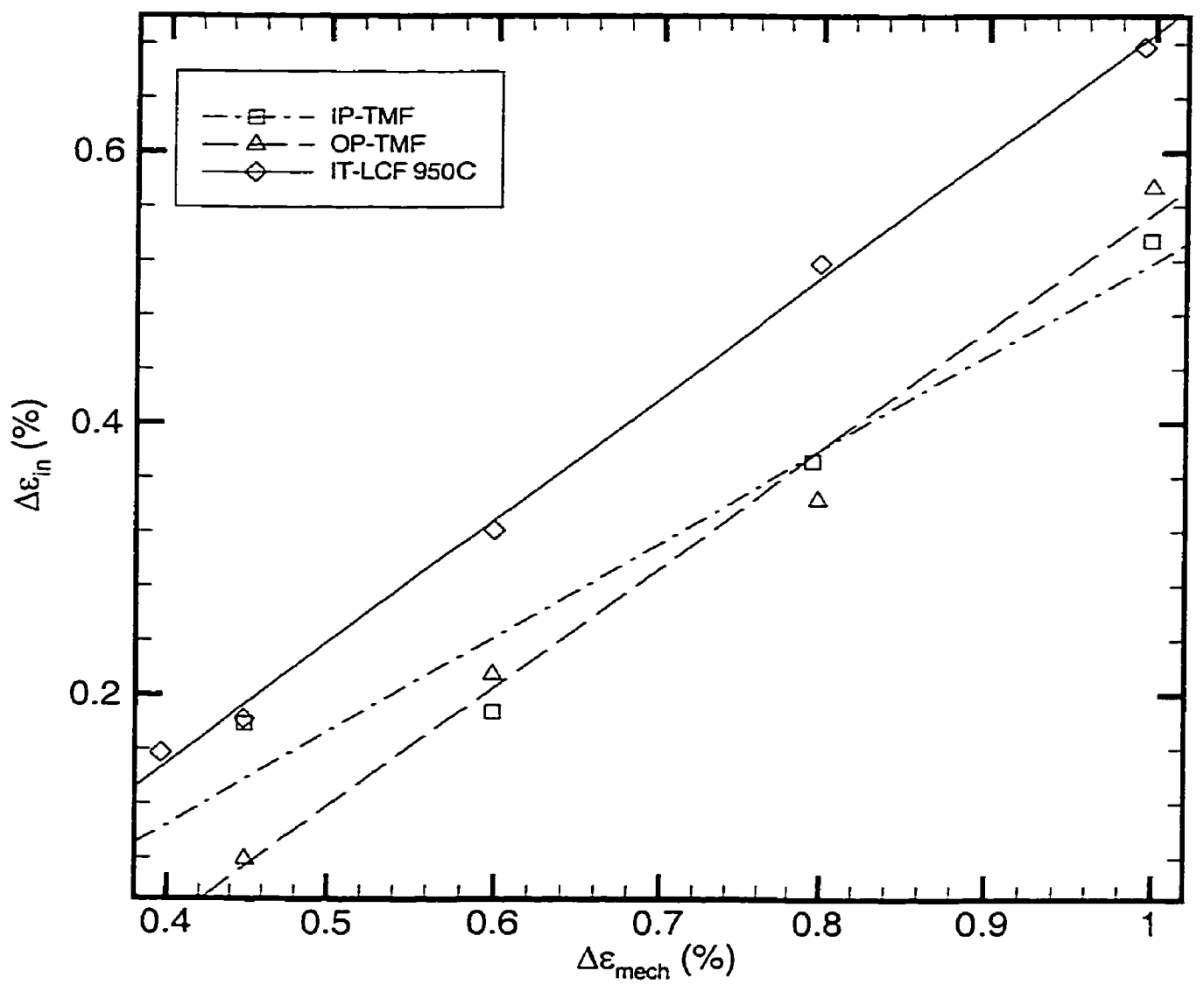

Figure 5-11 - Inelastic strain range plotted as a function of mechanical strain range for isothermal LCF, in-phase TMF and out-of-phase TMF tests. Note: the points shown on this figure are based on the average inelastic strain range for two or three tests. 

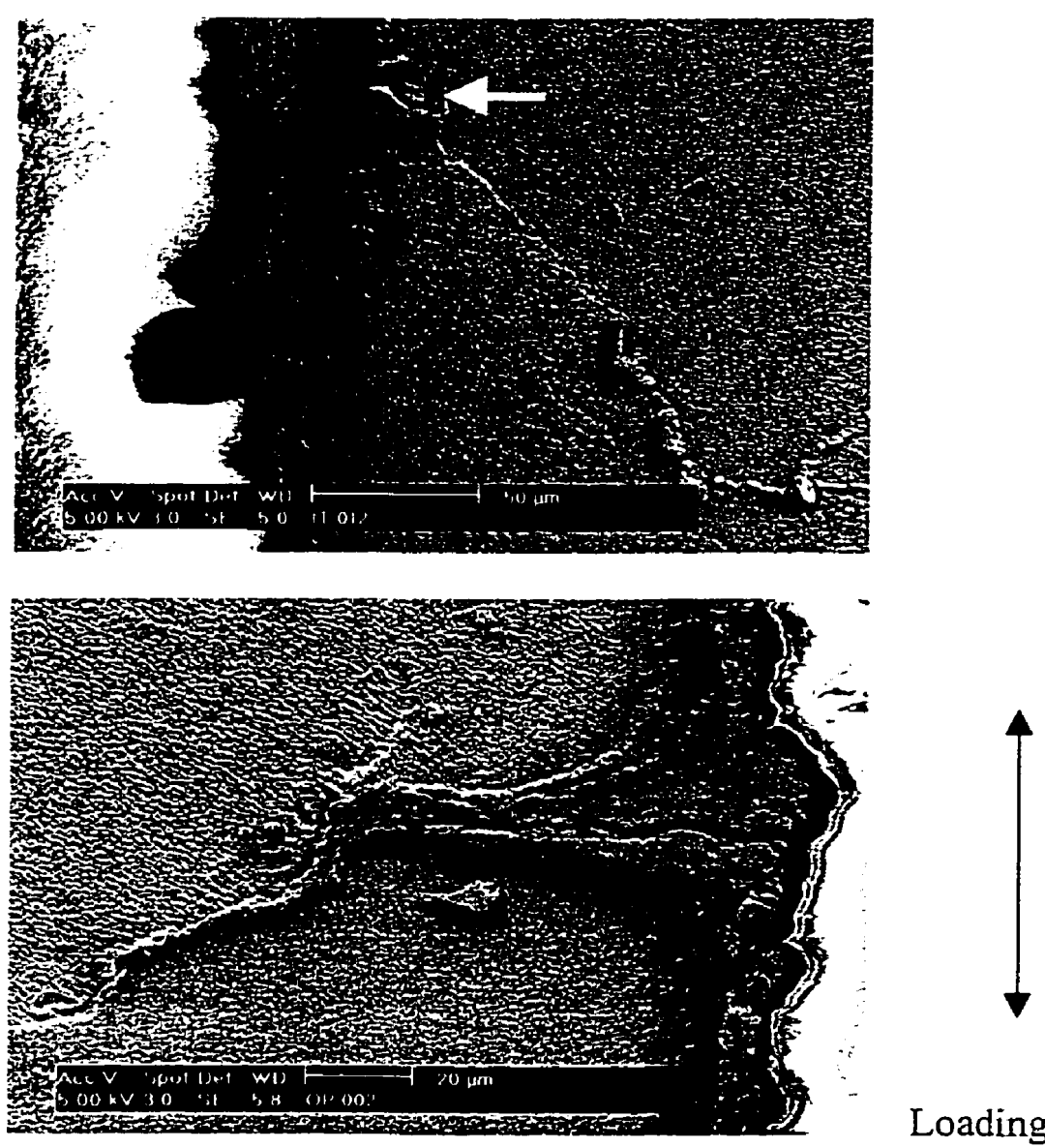

Loading Axis

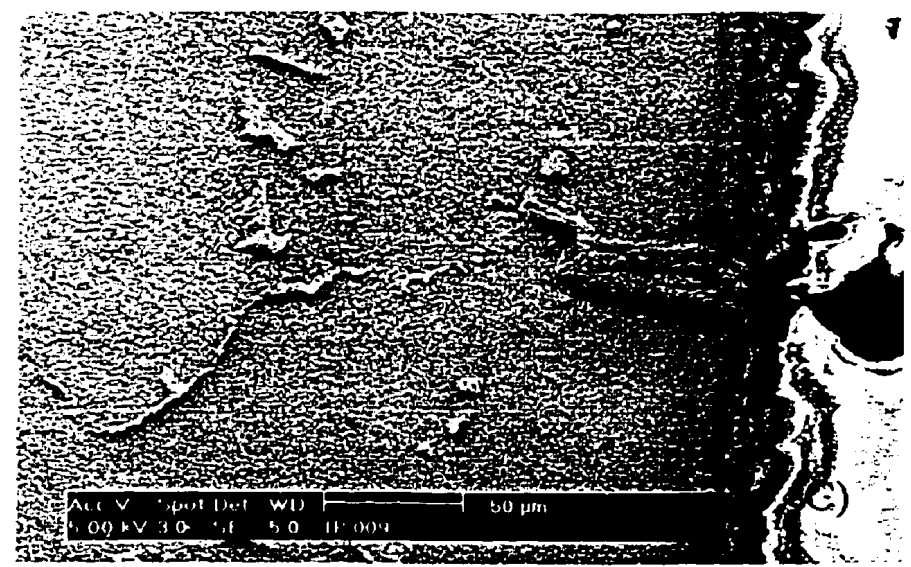

Figure 5-12 - Intergranular fatigue crack initiation sites observed in (A) isothermal-LCF (arrow), (B) out-of-phase TMF, and (C) in-phase TMF specimens. 

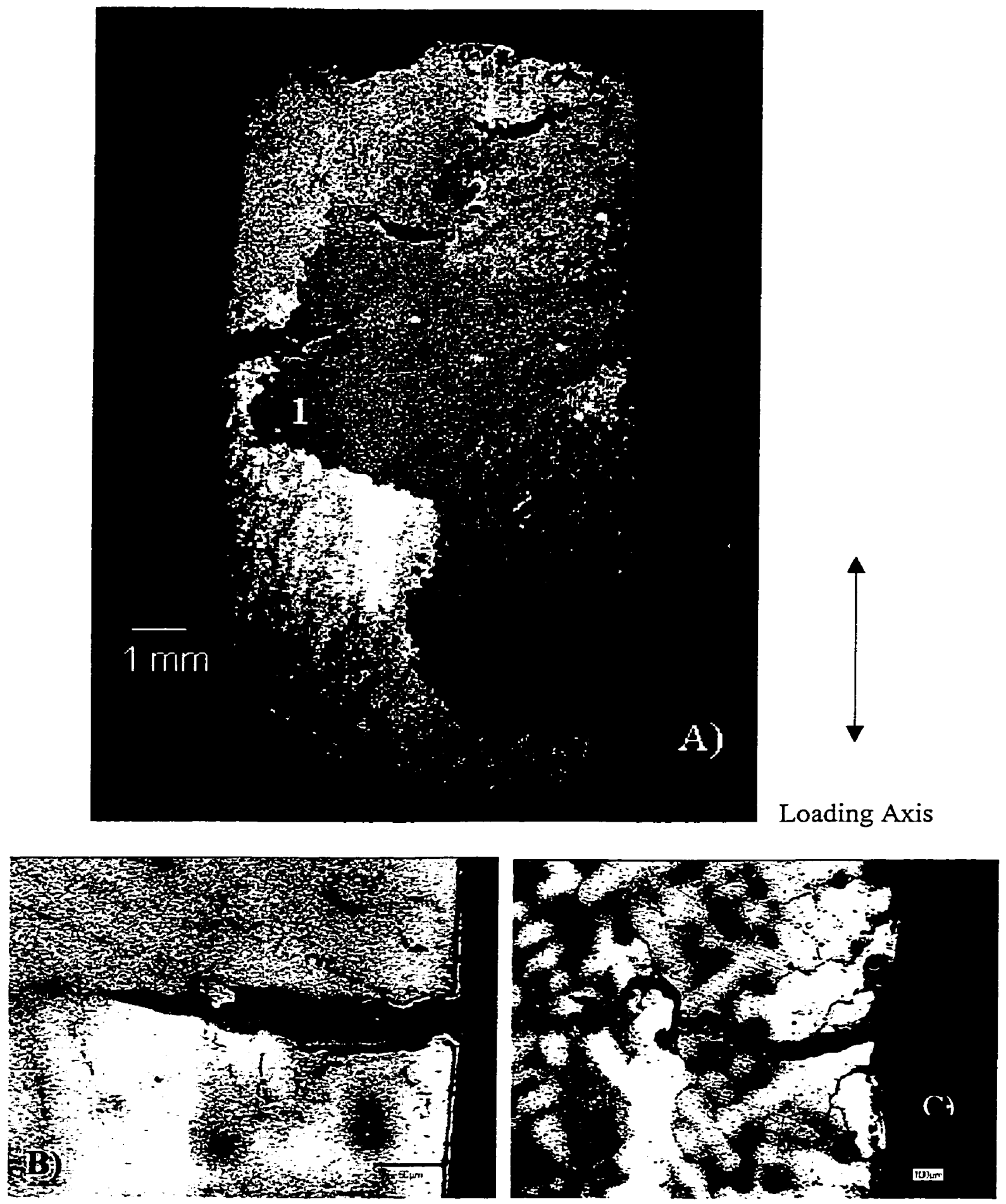

Figure 5-13 - (A) An example of (1) transgranular, and (2) intergranular fatigue crack propagation. (B) A close-up view of an intergranular fatigue crack, and (C) mixed intergranular-transgranular fatigue crack.propagation. 

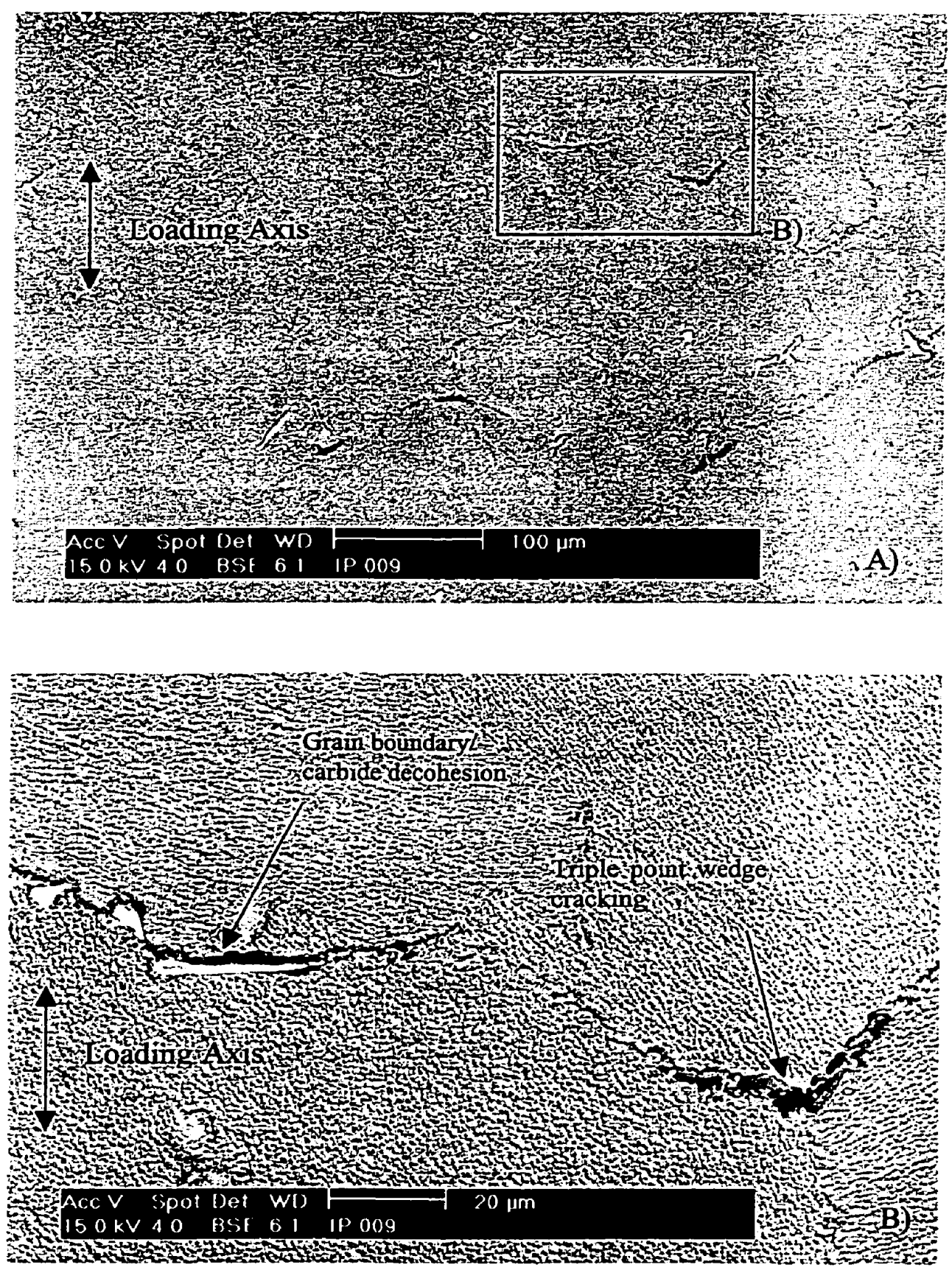

Figure 5-14 - (A) Micrograph illustrating intergranular damage observed in in-phase TMF tests, and B) enlarged view of region shown on (A) illustrating decohesion at carbide-grain boundary interface and triple point wedge cracking. 

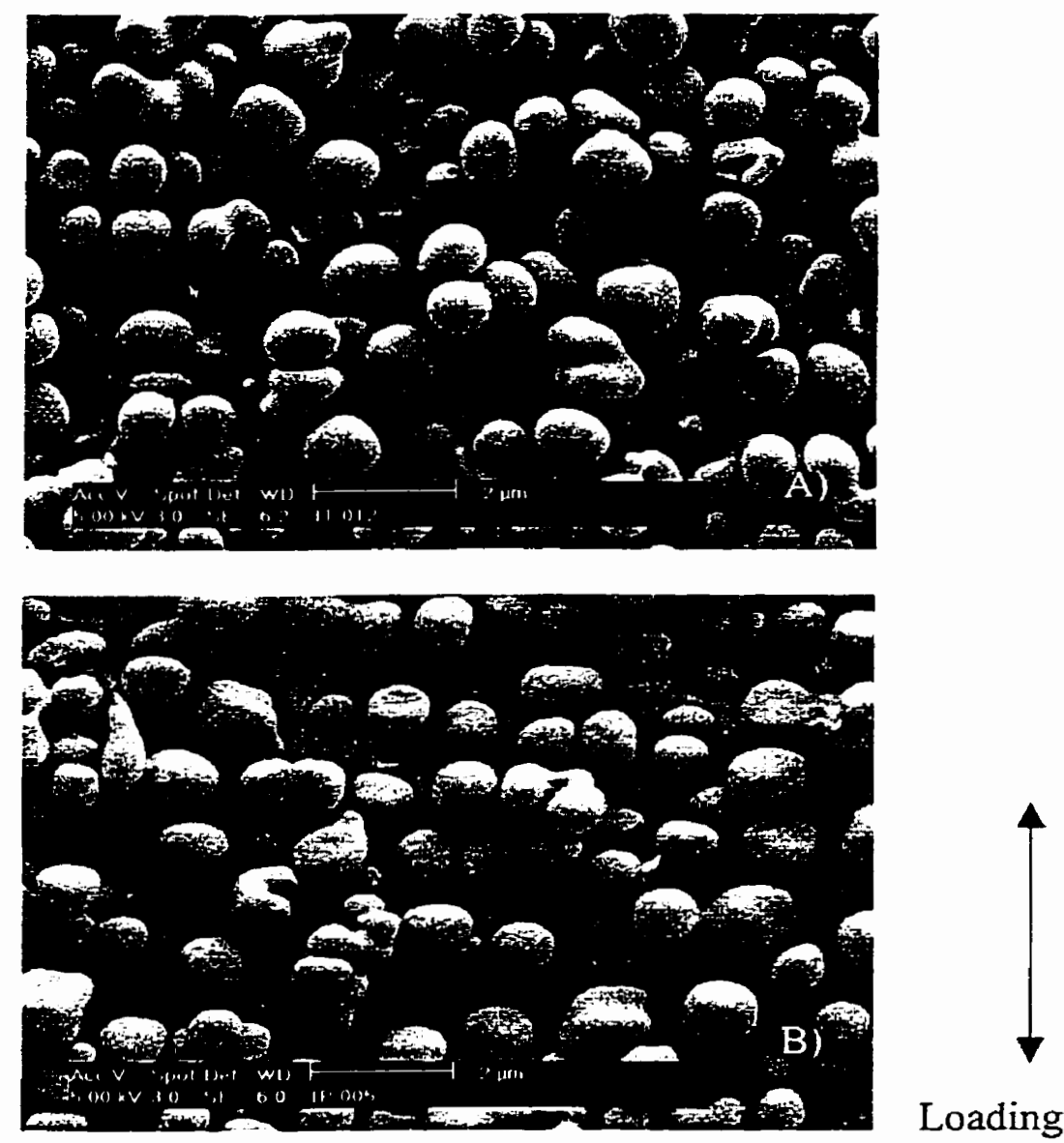

Loading Axis

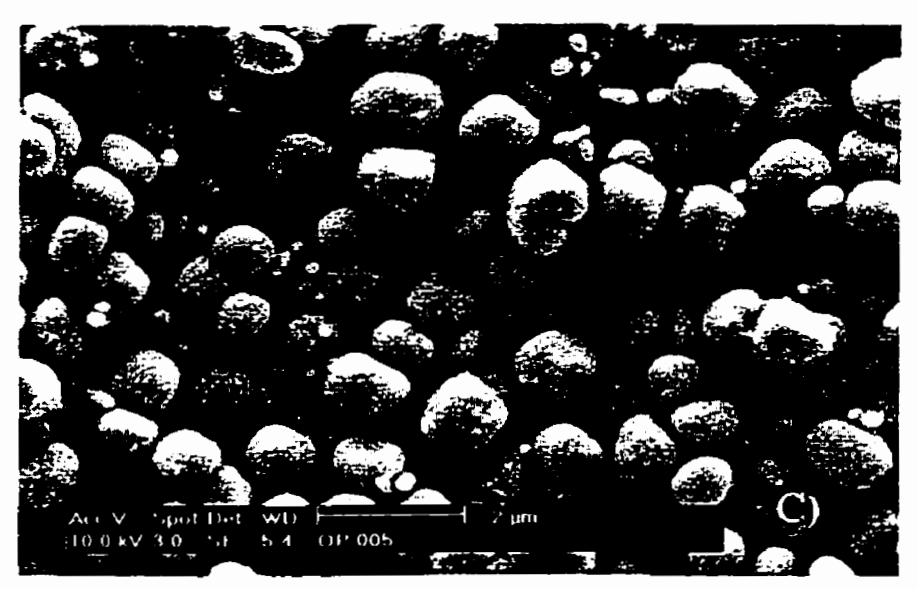

Figure 5-15 - Changes to $\gamma^{\prime}$ precipitate morphology after A) isothermal-LCF, B) in-phase TMF, and C) out-of-phase TMF cycling. 


\section{Summary and Recommendations for Future Work}

\subsection{Summary}

A dedicated facility for thermal-mechanical fatigue testing has been developed for the Institute for Aerospace Research-NRC and is fully operational. The TMF testing facility is composed of two independent closed loop control units; one loop for temperature control, the other for mechanical loading. Specimens are heated by an induction heating system with closed loop temperature control provided by a two-colour infrared pyrometer. Stable and accurate temperature control was realized by careful tuning of the induction heating coils and temperature control system. Testing procedures for IT-LCF and TMF testing were also developed in this work.

A dedicated data acquisition system and testing software were developed for synchronization of the mechanical loading and temperature control loops. The data acquisition and control software has been developed specifically for conducting fully reversed strain-controlled IT-LCF, and IP and OP-TMF tests in $600^{\circ} \mathrm{C}$ to $1000^{\circ} \mathrm{C}$ temperature range. The main features of the software are:

1. A user friendly interface for specification of test parameters and configuration of the data acquisition system,

2. Real-time display and data monitoring and, 
3. Modular design for increased versatility and to accommodate new testing requirements.

A series of commissioning IT-LCF and TMF tests on IN738LC, demonstrated the successful application of the testing procedures and proved the testing system was capable of cycling specimens under various programmed thermal-mechanical loading histories. In-phase and out-of-phase TMF commissioning tests were conducted with a constant mechanical strain rate of $2 \times 10^{-5} \mathrm{sec}^{-1}$, and with temperature cycling between $750^{\circ} \mathrm{C}$ to $950^{\circ} \mathrm{C}$. A series of IT-LCF tests were also conducted at $950^{\circ} \mathrm{C}$ and at the same mechanical strain rate as the TMF tests for comparison purposes. The following conclusions can be drawn from the results of these tests:

1. Fatigue life differs with strain-temperature phasing and strain range,

2. The results of the IT-LCF tests provide a conservative estimate of cyclic life under IP-TMF loading for the mechanical strain ranges considered in this investigation,

3. Crack initiation and propagation occurred predominantly at grain boundaries that intersect with the specimen surface, and

4. IP-TMF tests showed a significant creep component of damage.

\subsection{Recommendations for Future Work}

- Presently, the TMF facility developed as part of this thesis research is limited to fully reversed, IP and OP-TMF fatigue tests, without forced air cooling. Additional 
capability could be added for arbitrary, non-proportional-type, temperature-strain relationships, with non-zero mean strain values. Specimen cooling capability could also be added to increase the testing frequency. This would be especially useful for TMF tests conducted on single crystal or directionally solidified materials, which tend to have considerably longer fatigue lives than conventionally cast materials.

- Additional testing with IN738LC could be performed to further investigate the damage mechanisms operative under in-phase and out-of-phase TMF loading. By conducting additional tests, which would be interrupted at various portions of fatigue life at the same mechanical strain ranges considered in this thesis, further insight into the relative contributions of creep, oxidation, and fatigue to the overall damage process could be studied. This additional work may also make it possible to support the development of a life prediction model for $\mathbb{N} 738 \mathrm{LC}$, in the temperature range considered in this thesis.

- Further metallographic examination using transmission electron microscopy (TEM) is necessary to investigate the dislocation mechanisms operative under in-phase and outof-phase TMF, and isothermal LCF loading conditions. TEM investigation may reveal the dislocation-precipitate interactions, which dictate the cyclic hardeningsoftening and deformation behavior of the material, operative under each loading condition. The results of this investigation may also provide support required for developing a deformation model for N738LC under TMF and LCF loading conditions. 


\section{References}

' Sims, C. T., "Superalloys: Genesis and Characters," in Superalloys I, C. T. Sims, et al., Eds., John Wiley \& Sons, New York, 1987, pp. 3-26.

2 Poole, J.M., Fischer, J.J., Hack, G.A.J., and McColvin, G.M., "The Development , Performance and Future of the Mechanical Alloying Process and Oxide Dispersion Strengthened Alloys," in Advances in High Temperature Structural Materials and Protective Coatings, A.K. Koul, et al., Eds., National Research Council of Canada Publications, Ottawa, 1994, pp. 32-53.

3 “The Jet Engine," 5 ${ }^{\text {th }}$ Ed., Derby, U.K., Rolls-Royce Plc., 1996.

${ }^{4}$ Harrison, G.F., Tranter, P.H., Williams, S.J., "Modeling of Thermomechanical Fatigue in Aero Engine Turbine Blades," in Thermal-Mechanical Fatigue of Aircraft Engine Materials, AGARD Conference Proceedings 569, AGARD-CP-569, Paper No. 16, pp. 112.

5 Miller, H.E., and Chambers, W.L., "Gas Turbine Design and Superalloys," in Superalloys $\amalg$, C. T. Sims, et al., Eds., John Wiley \& Sons, New York, 1987, pp. 27-60.

6 Jones, W.B., Schmale, D.T., and Bourcier, R.J., "A Test System for Computer Controlled Thermomechanical Fatigue Testing," in Applications of Automation Technology to Fatigue and Fracture Testing, ASTM STP 1092, A.A. Braun, N. E. Ashbaugh, and F. M. Smith, Eds., American Society for Testing and Materials, Philadelphia, 1990, pp. 38-51.

${ }^{7}$ Guedou, J.-Y., and Honnorat, Y., "Thermomechanical Fatigue of Turbo-Engine Blade Superalloys," in Thermomechanical Fatigue Behavior of Materials, ASTM STP 1186, H. Sehitoglu, Ed., American Society for Testing and Materials, Philadelphia, 1993, pp. 157175.

${ }^{8}$ Fleury, E., and Remy, L, "Behavior of Nickel-Base Superalloy Single Crystals under Thermal-Mechanical Fatigue," Metallurgical and Materials Transactions A, Vol. 25A, January 1994, pp. 99-109.

${ }^{9}$ Nitta, A., Kuwabara, K., and Kitamura, T., "The Characteristics of Thermal-Mechanical Fatigue Strength in Superalloys for Gas Turbine," Central Research Institute of Electric Power Industry, Energy and Environment Laboratory, CRIEPI Report E282011.

10 Remy, L., "Thermal-Mechanical Fatigue and Thermal Fatigue Experiments," in Mechanical Behavior of Materials at High Temperature, Kluwer Academic Publisher, Netherlands, 1996, pp. 381-398. 
Spera, D.A., "What is Thermal Fatigue?," in Thermal Fatigue of Materials and Components, ASTM STP 612, D.A. Sperá and D.F. Mowbray, Eds., American Society for Testing and Materials, 1976, pp 3-9.

12 Spera, D. A., Calfo, F.D, and Bizon, P.T., "Thermal Fatigue Testing of Simulated Turbine Blades," SAE National Air Transportation Meeting, Atlanta GA., Paper 710459, Society of Automotive Engineers, New York, 1971.

${ }^{13}$ Malpertu, J. L., and Remy, L., "Thermomechanical Fatigue Behavior of a Superalloy," in Low Cycle Fatigue, ASTM STP 942, H.D. Solomon, G. R. Halford, L. R. Kaisand, and B. N. Leis, Eds., American Society for Testing and Materials, Philadelphia, 1988, pp. 657-671.

${ }^{14}$ Hammond, C., and Nutting, J., "The Physical Metallurgy of Superalloys and Titanium Alloys," Forging and the Properties of Aerospace Materials, Paper No. MS 627, TMS, January 1977.

${ }^{15}$ Bradley E.F., Ed., "Superalloys: A Technical Guide," ASM International, 1988.

${ }^{16}$ Sims, C. T., "Nickel Alloys - The Heart of Gas Turbine Engines," Paper 70-GT-24, ASME, 1970.

${ }^{17}$ Hertzberg, R.W., "Deformation and Fracture of Mechanics of Engineering Materials," $2^{\text {nd }}$ Ed., John Wiley \& Sons, New York, 1983.

${ }^{18}$ Bannantine, J. A., Comer, J. J., and Handrock, J. L., "Fundamentals of Metal Fatigue Analysis," Prentice-Hall, New Jersey, 1990.

${ }^{19}$ Miner, R. V., "Fatigue," in Superalloys II, High-Temperature Materials for Aerospace and Industrial Power, C. T. Sims, N. S. Stoloff, W. C. Hagel, Eds., John Wiley and Sons Inc., New York, 1987, pp. 263-289.

${ }^{20}$ Raske, D. T., and Morrow, JoDean, "Mechanics of Materials in Low Cycle Fatigue Testing," in Manual on Low Cycle Fatigue Testing, ASTM STP 465, American Society for Testing and Materials, 1969, pp. 1-25.

21 Manson, S.S., "Thermal Stress and Low-Cycle Fatigue", McGraw-Hill, Inc., New York, 1966

${ }^{22}$ Coffin, L. F., Jr., "Fatigue at High Temperature," in Fatigue at Elevated Temperatures, ASTM STP 520, American Society for Testing and Materials, 1973, pp. 5-34.

${ }^{23}$ Runkle, J. C., and Pelloux, R. M., "Micromechanisms of Low-Cycle Fatigue in NickelBased Superalloys at Elevated Temperatures," in Fatigue Mechanisms. Proceedings of an ASTM-NBS-NSF Symposium, Kansas City, Mo., May 1978, J. T. Fong, Ed., ASTM STP 675, American Society for Testing and Materials, 1979, pp. 501-527. 
24 Halford, G.R., "High Temperature Fatigue in Metals," Advanced Materials Technology, CP-2251, Nov. 1982.

${ }^{25}$ Gell, M. and Leverant, G.R., "Mechanisms of High-Temperature Fatigue," in Fatigue at Elevated Temperatures, ASTM STP 520, American Society for Testing and Materials, 1973, pp. 37-67.

${ }^{26}$ Ostergren, W. J., "A Damage Function and Associated Failure Equations for Predicting Hold Time and Frequency Effects in Elevated Temperature, Low Cycle Fatigue," Journal of Testing and Evaluation, JTEVA, Vol. 4, No. 5, September 1976, pp. 327-339.

${ }^{27}$ Coffin, L. F., Jr., "The Effect of Frequency on the Cyclic Strain and Fatigue Behavior of Cast Rene at $1600^{\circ} \mathrm{F}, "$ Metallurgical Transactions, Vol. 5, May 1974, pp. 1053-1060.

28 Antolovich, S. D., Baur, R., and Liu, S., "A Mechanistically Based Model For High Temperature LCF of Ni-Base Superalloys," Superalloys 1980, TMS-AIME, pp. 605-613.

29 Remy, L., Bernard, H., Malpertu, J. L., and Rezai-Aria, F., "Fatigue Life Prediction Under Thermal-Mechanical Loading in a Nickel-Base Superalloy," in Thermomechanical Fatigue Behavior of Materials, ASTM STP 1186, H. Sehitoglu, Ed., American Society for Testing and Materials, Philadelphia, 1993, pp. 3-16.

30 Reuchet, J., and Remy, L., "High Temperature Low Cycle Fatigue of Mar-M 509 Superalloy II: The Influence of Oxidation at High Temperatures," Materials Science and Engineering, Vol. 58, No. 1, March 1983, pp. 33-42.

31 Reuchet, J., and Remy, L., "Fatigue Oxidation Interaction in a Superalloy Application to Life Prediction in High Temperature Low Cycle Fatigue," Metallurgical Transactions A, TMS-AIME, Vol. 14A, January 1983, pp. 141-149.

32 Nazmy, M.Y., "The Effect of Environment on the High Temperature Low Cycle Fatigue Behavior of Cast Nickel-base IN-738 Alloy," Materials Science and Engineering, Vol. 52, September 1982, pp. 231-237.

33 Gendron, S., "On-Line Assessment and Numerical Modelling of Thermal Fatigue and Oxidation Damages in Turbine Airfoils Under Simulated Conditions," Ph.D. thesis, Ecole Polytechnique, Montreal, 1996.

34 Koster, A., Fleury, E., Vasseur, E., and Remy, L., "Thermal-Mechanical Fatigue Testing," in Automation in Fatigue and Fracture: Testing and Analysis, ASTM STP 1231, C. Amzallag, Ed., American Society for Testing and Materials, Philadelphia, 1994, pp. 563-580.

35 Beck, T., Pitz, G., Lang, K.-H., Lohe, D., "Thermal-Mechanical and Isothermal Fatigue of IN 792 CC," Materials Science and Engineering A, Vol. 234, 1997, 719-722. 
${ }^{36}$ Bill, R. C., Verrilli, M. J., McGaw, M. A., and Halford, G. R., "Preliminary Study of Thermomechanical Fatigue of Polycrystalline Mar-M 200," AVSCOM Technical Report 83-C-6, 1984.

${ }^{37}$ Pototzky, P., Maier, H.J., and Christ, H.-J., "Thermomechanical Fatigue Behavior of the High-Temperature Titanium Alloy IMI 834," Metallurgical and Materials Transactions A, Vol. 29A, December 1998, pp. 2995-3004.

38 Sheffler, K.D., "Vacuum Thermal-Mechanical Fatigue Behavior of Two Iron-Base Alloys," in Thermal Fatigue of Materials and Components, ASTM STP 612, D.A. Spera and D.F. Mowbray, Eds., American Society for Testing and Materials, 1976, pp. 214226.

39 Komenda, J., Linde, L. and Henderson, P.J., "Microstructural Aspects of Damage Occurring During Thermo-mechanical and Low Cycle Fatigue Testing of an Oxide Dispersion Strengthened Alloy," in Fatigue Under Thermal and Mechanical Loading, J. Bressers and L. Remy, Eds., Kluwer Academic Publishers, Netherlands, 1996, pp. 339347.

40 Kleinpass, B., Lang, K.-H., Lohe, D., and Macherauch, E., "Thermal-Mechanical Fatigue Behavior of NiCr22Col2Mo9," in Fatigue Under Thermal and Mechanical Loading, J. Bressers, and L. Remy, Eds., Kluwer Academic Publishers, Netherlands, 1996, pp. 327-337.

${ }^{41}$ Chen, W., Dudka, A., Chen, H., Mukherji, R.P., Wahi, R P., and Wever, H., "Damage and Fatigue Life of Superalloy IN738LC Under Thermo-Mechanical and Low Cycle Fatigue Loading," in Fatigue Under Thermal and Mechanical Loading, J. Bressers and L. Remy, Eds., Kluwer Academic Publishers, Netherlands, 1996, pp. 97-102.

42 Boismier, D. A., and Sehitoglu, H., "Thermo-Mechanical Fatigue of Mar-M247: Part 1 - Experiments," Journal of Engineering Materials and Technology, Trans. ASME, American Society of Mechanical Engineers, Vol. 112, January 1990, pp. 68-79.

43 Marchand, N., L'Esperance, G., and Pelloux, R.M., "Thermal-Mechanical Cyclic Stress-Strain Reponses of Cast B-1900 + Hf," Low Cycle Fatigue, ASTM STP 945, H.D. Solomon, G.R. Halford, L.R. Kaisand, and B.N. Leis, Eds., American Society for Testing and Materials, Philadelphia, 1988, pp. 638-656.

44 Reger, M. and Remy, L., "Fatigue Oxidation Interaction in IN 100 Superalloy," Metallurgical Transactions A, Vol. 19A, September 1988, pp. 2259-2268.

45 Wahi, R.P., Auerswald, D., Mukherji, D., Dudka, A., Fecht, H.-J., and Chen, W., "Damage Mechanisms of Single and Polycrystalline Nickel Base Superalloys SC16 and IN738LC Under High Temperature LCF Loading," Intl. Journal of Fatigue, Vol. 19, No. 1, Supplement, pp. S89-S94. 
46 Jianting, G., Ranucci, D., and Picco, E., "Low Cycle Fatigue Behavior of Cast Nickelbase Superalloy IN-738LC in Air and in Hot Corrosive Environments," Materials Science and Engineering, Vol. 58, March 1983, pp. 127-133.

${ }^{47}$ Kadioglu, Y., Sehitoglu, H., "Thermomechanical and Isothermal Fatigue Behavior of Bare and Coated Superalloys," Journal of Engineering Materials and Technology, Trans. ASME, American Society of Mechanical Engineers, Vol. 117, No. 1, July 1995, pp. 94102.

48 Sehitoglu, H. and Boismier, D., "Thermo-Mechanical Fatigue of Mar-M247: Part 2 Life Prediction," Journal of Engineering Materials and Technology, Trans. ASME, American Society of Mechanical Engineers, Vol. 112, January 1990, pp. 80-88.

49 Reuchet, J., and Remy, L., "High Temperature Low Cycle Fatigue of Mar-M 509 Superalloy I: The Influence of Temperature on the Low Cycle Fatigue Behavior from 20 to $1100^{\circ} \mathrm{C}$," Materials Science and Engineering, Vol. 58, No. 1, March 1983, pp. 19-32.

${ }^{50}$ Antolovich, S.D., Liu, S., and Baur, R., "Low Cycle Fatigue Behavior of Rene 80 at Elevated Temperature," Metallurgical Transactions A, Vol. 12A, March 1981, pp. 473481.

${ }^{51}$ Wright, P.K., "Oxidation-Fatigue Interactions in a Single-Crystal Superalloy," in Low Cycle Fatigue, ASTM STP 942, H.D. Solomon, G.R. Halford, L.B. Kaisand, and B.N. Leis, Eds., American Society for Testing and Materials, Philadelphia, 1988, pp. 558-575.

52 Wright, P.K., and Anderson, A.F., "The Influence of Orientation on the Fatigue of Directionally Solidified Superalloys," Superalloys 1980, TMS-AIME, pp. 689-698.

53 Engler-Pinto, C.C., Jr., Meyer-Olbersleben, F., and Rezai-Aria, F., "ThermoMechanical Fatigue Behavior of SRR99," in Fatigue Under Thermal and Mechanical Loading, J. Bressers and L. Remy, Eds., Kluwer Academic Publishers, Netherlands, 1996, pp. 151-157.

54 Bizon, P.T., and Spera, D.A., "Thermal-Stress Fatigue Behavior of Twenty-Six Superalloys," in Thermal Fatigue of Materials and Components, ASTM STP 612, D.A. Spera and D.F. Mowbray, Eds., American Society for Testing and Materials, 1976, pp. 106-122.

${ }^{55}$ Manson, S.S., Halford, G.R., and Hirshberg, M.H., "Creep-Fatigue Analysis by StrainRange Partitioning," NASA Technical Memorandum, NASA TM X-67838, 1971.

56 Henderson, P.J., "The Use of Strain Range Partitioning in Thermo-Mechanical Fatigue," Scripta Materialia, Vol. 34, No. 12, 1996, pp. 1839-1844.

57 Manson, S.S., and Halford, G.R., "Relation of Cyclic Loading Pattern to Microstructural Fracture in Creep-Fatigue," Fatigue 84, pp. 1237-1255. 
58 Halford, G.R., and Manson, S.S., "Life Prediction of Thermal-Mechanical Fatigue Using Strainrange Partitioning," in Thermal Fatigue of Materials and Components, ASTM STP 612, D.A. Spera and D.F. Mowbray, Eds., American Society for Testing and Materials, 1976, pp. 239-254.

59 Bui-Quoc, T., Gomuc, R., Biron, A., Nguyen, H.L., and Masounave, J., "Elevated Temperature Fatigue-Creep Behavior of Nickel-Base Superalloy IN 625," Low Cycle Fatigue, ASTM STP 942, H.D. Solomon, G.R. Halford, L.R. Kaisand, and B.N. Leis, Eds., American Society for Testing and Materials, Philadelphia, 1988, pp. 470-486.

60 "Influence of Grain Size, Strain Range and Creep Ductility on Creep-Fatigue Interaction," Progress in Materials Science, Vol. 37, No. 5, 1993, pp. 436-480.

${ }^{61}$ Neu, R.W., and Sehitoglu, H., "Thermomechanical Fatigue, Oxidation, and Creep: Part 1. Damage Mechanisms," Metallurgical Transactions A, Vol. 20A, No. 9, September 1989, pp. 1755-1767.

62 Pettit, F.S., and Meier, G.H., "Oxidation and Hot Corrosion of Superalloys," Superalloys 1984, TMS-AIME, pp. 651-687.

${ }^{63}$ Webb, G., et al., "Prediction of Oxidation Assisted Crack Growth Behavior within Hot Section Gas Turbine Components," Superalloys 1996, R. D. Kissinger et al., Eds., TMSAIME, 1996, pp. 345-352.

${ }^{64}$ Smialek, J.L., and Meier, G.H., "High-Temperature Oxidation," in Superalloys II, High-Temperature Materials for Aerospace and Industrial Power, C. T. Sims, N. S. Stoloff, W. C. Hagel, Eds., John Wiley and Sons Inc., New York, 1987, pp. 293-326.

${ }^{65}$ Esmaeili, S., Engler-Pinto, C.C., Jr., Ilschner, B., and Rezai-Aria, F., "The Effect of Thermo-Mechanical Fatigue Loading On Surface Oxidation of IN738LC Superalloy," in Fatigue Under Thermal and Mechanical Loading, J. Bressers, and L. Remy, Eds., Kluwer Academic Publishers, Netherlands, 1996, pp. 103-108.

${ }^{66}$ Esmaeili, S., Engler-Pinto, C.C., Jr., Ilschner, B., and Rezai-Aria, F., "Interaction Between Oxidation and Thermo-Mechanical Fatigue In IN738LC Superalloy-I," Scripta Materialia, Vol. 32, No. 11, 1995, pp. 1777-1781.

${ }^{67}$ Skelton, R.P., and Bucklow, J.I., "Cyclic Oxidation and Crack Growth During High Strain Fatigue of Low Alloy Steel," Metal Science, Vol. 12, 1978, pp. 64-70.

68 Hancock, P., and Nicholls, J.R., "Failure of Oxide Scales," in Materials at High Temperatures Special Issue Mechanical Properties of Oxide Scales, Vol. 12, Nos. 2-3, 1994, pp. 209-218.

69 Manning, M.I., "Geometrical Effects of Oxide Scale Integrity," Corrosion Science, Vol. 21, No. 4, Pergamon Press, U.K., 1981, pp. 301-316. 
${ }^{70}$ Remy, L., "Oxidation Effects in High Temperature Creep and Fatigue on Engineering Alloys," International Conference on Corrosion-Deformation Interactions, CDI'92, Fontainebleau, France, October 1992, pp. 425-459.

${ }^{71}$ Marchand, N.J., and Dorner, W., "Thermal Fatigue Damage in cast IN-100 Superalloy: Experiments and Modelling," Materials at High Temperatures, Vol. 9, No. 4, November 1991, pp. 217-227.

72 Gell, M., and Duquette, D.J., "The Effects of Oxygen on Fatigue Fracture of Engineering Alloys," Conference Proceedings, Corrosion Fatigue: Chemistry, Mechanics and Microstructure: June 14-18, 1971, University of Connecticut, Storrs, Connecticut, pp. 366-378.

73 Pineau, A., "Fatigue and Creep-Fatigue Behavior of Ni-Base Superalloys: Microstructural and Environmental Effects," in Mechanical Behavior of Materials at High Temperature, C. Moura Branco, et al., Eds., Kluwer Academic Publishers, Netherlands, 1996, pp. 135-154.

${ }^{74}$ Neu, R.W., and Sehitoglu, H., "Thermomechanical Fatigue, Oxidation, and Creep: Part I. Damage Mechanisms," Metallurgical Transactions A, Vol. 20A, No. 9, September 1989, pp. 1755-1767.

${ }^{75}$ Neu, R.W., and Sehitoglu, H., "Thermomechanical Fatigue, Oxidation, and Creep: Part II. Life Prediction," Metallurgical Transactions A, Vol. 20A, No. 9, September 1989, pp. 1769-1783.

${ }^{76}$ Castelli, M.G., Miner, R.F., and Robinson, D.N., "Thermomechanical Deformation Behavior of a Dynamic Strain Aging Alloy, Hastelloy X," in Thermomechanical Fatigue Behavior of Materials, ASTM STP 1186, H. Sehitoglu, Ed., American Society for Testing and Materials, Philadelphia, 1993, pp. 106-125.

77 Sehitoglu, H., "Thermo-Mechanical Deformation of Engineering Alloys and Composites - Experiments and Modeling," in Mechanical Behavior of Materials at High Temperature, C. Moura Branco et al., Eds., Kluwer Academic Publishers, Netherlands, 1996, pp. 349-379.

78 Marchand, N. and Pelloux, R.M., "A Computerized Test System for ThermalMechanical Fatigue Crack Growth," Journal of Testing and Evaluation, JTEVA, Vol. 14, No. 6, Nov. 1986, pp. 303-311.

79 Pernot, J.J. and Mall, S., "A Thermal-Mechanical Fatigue Crack-Growth Testing System," Society for Experimental Mechanics, Vol. 13, No. 2, February 1989, pp. 24-28.

80 Carden, A. E., "Thermal Fatigue Evaluation," in Manual on Low Cycle Fatigue Testing, ASTM STP 465, American Society for Testing and Materials, 1970, pp. 163 188. 
81 Verrilli, M.J., Castelli, M.G., Bressers, J., and Oehmke, R.L.T., "Standardization Activities in TMF Test Methodologies," in Thermal Mechanical Fatigue of Aircraft Engine Materials, AGARD-CP-569, 1995, pp. 4 (1-11).

82 "Standard Practice for Strain Controlled Fatigue Testing", ASTM Designation: E60692, Section 3, Vol. 03.01, 1992, pp. 525-539.

83 Aksoy, S.Z., Gayda, J., and Gabb, T.P. "Fatigue Behavior of [O] $]_{8}$ SCS-6/Ti-6Al-4V Composite Subjected to High Temperature Turboshaft Design Cycles," in Thermomechanical Fatigue Behavior of Materials: Second Volume, ASTM STP 1263, M.J. Verrilli, and M.G. Castelli, Eds., American Society for Testing and Materials, 1996, pp. 266-279.

84 Shi, H., Korn, C., and Pluvinage, G., "High Temperature Isothermal and Thermomechanical Fatigue on a Molybdenum-based Alloy", Materials Science and Engineering A, Vol. 247, Elsevier Science S.A., 1998, pp. 180-186.

85 Berling, J.T., and Slot, T., "Effect of Temperature and Strain Rate on Low-Cycle Fatigue Resistance of AISI 304, 316, and 348 Stainless Steels," in Fatigue at High Temperature, ASTM STP 459, American Society for Testing and Materials, 1969, pp. 330.

${ }^{86}$ Ellison, E.G., and Lohr, R.D., "The Extensometer-Specimen Interface," in Techniques for High Temperature Fatigue Testing. G. Sumner and V.B. Livesey, Eds. Elsevier Applied Science Publishers, London, 1983, pp. 1-28.

87 McCarthy, P. "Taking the Test: In Thermomechanical Fatigue," Materials World, 1994, Vol. 2, No. 9, pp. 468-470.

${ }^{88}$ Halford, G.R., McGaw, M.A., Bill, R.C., and Fanti, P.D., "Bithermal Fatigue: A Link Between Isothermal and Thermomechanical Fatigue," in Low Cycle Fatigue, ASTM STP 942, H.D. Solomon, G.R. Halford, L.R. Kaisand, and B.N. Leis, Eds., American Society for Testing and Materials, Phialdelphia, 1988, pp. 625-637.

${ }^{89}$ Castelli, M.G., and Ellis, J.R., "Improved Techniques for Thermomechanical Testing in Support of Deformation Modelling", in Thermomechanical Fatigue Behavior of Materials, ASTM STP 1186, H. Sehitoglu, Ed. ASTM Publications, Philadelphia, PA, 1993, pp 195-211.

${ }^{90}$ Lindholm, U.S., and Davidson, D.L., "Low-Cycle Fatigue with Combined Thermal and Strain Cycling," in Fatigue at Elevated temperatures, ASTM STP 520, American Society for Testing and Materials, 1973, pp. 473-481.

91 Jaske, C.E., "Thermal-Mechanical, Low-Cycle Fatigue of AISI 1010 Steel," in Thermal Fatigue of Materials and Components, ASTM STP 612, D. A. Spera and D. F. Mowbray, Eds., American Society for Testing and Materials, 1976, pp. 170-198. 
92 "Standard Test Method for Strain Controlled Thermomechanical Fatigue Testing," TMF Working Document, 11/99, ASTM Thermomechanical Fatigue Task Group, E08.05.07.

93 Dai, Y., "Thermal-Mechanical Fatigue Crack Growth in Aircraft Engine Materials," Ph.D. thesis, Ecole Polytechnique, Montreal, 1993.

${ }^{94}$ Zamrik, S.Y., Davis, D.C., and Firth, L.C., "Isothermal and Thermomechanical Fatigue of Type 316 Stainless Steel," in Thermomechanical Fatigue Behavior of Materials: Second Volume, ASTM STP 1263, M.J. Verilli and M.G. Castelli, Eds., American Society for Testing and Materials, 1996 pp. 96-116.

${ }^{95}$ Hopkins, S.W., "Low-Cycle Thermal Mechanical Fatigue Testing," in Thermal Fatigue of Materials and Components, ASTM STP 612, D.A. Spera and D.F. Mowbray, Eds., American Society for Testing and Materials, 1976, pp. 157-169.

96 Carden, A.E., "Fatigue at Elevated Temperatures: A Review of Test Methods," in Fatigue at Elevated Temperatures, ASTM STP 520, American Society for Testing and Materials, 1973, pp. 195-223.

${ }^{97}$ Pettit, F.S., Goward, G.W., "High Temperature Corrosion and Use of Coating for Protection," in Metallurgical Trestises, J.K. Tien and J.F. Elliot, Eds., TMS-AIME 1981, pp. 603-619.

${ }^{98}$ Davies, J., and Simpson, P., "Induction Heating Handbook," McGraw-Hill, UK, 1979.

${ }^{99}$ Coffin, L. F., Jr., "The Stability of Metals Under Cyclic Plastic Strain," Transactions of the ASME, Journal of Basic Engineering, Vol. 82D, Sept. 1960, pp. 671-682.

${ }^{100}$ Chen, H. et al., "Cyclic Life of Superalloy IN738LC Under In-Phase and Out-ofPhase Thermo-mechanical Fatigue Loading," Zeitschrift fur Metallkunde, Vol. 86, No. 6, 1999 , pp. 423-427.

101 Kandil, F.A., "Potential Ambiguity in the Determination of the Plastic Strain Range Component in LCF Testing," International Journal of Fatigue, Vol. 21, Elsevier, 1999, pp. 1013-1018.

${ }^{102} \mathrm{Au}, \mathrm{P}$. , "Development of an Isothermal Low Cycle Fatigue Testing Facility", NAE, NRC-LTR-ST-1601, Sept. 1986.

${ }^{103}$ Raynor, D., and Skelton, R.P., "The Onset of Cracking and Failure Criteria in High Strain Fatigue," in Techniques for High Temperature Fatigue Testing, G. Sumner and V.B. Livesey, Eds. Elsevier Applied Science Publishers, London, 1983, pp. 143-166.

104 Rao, K.B.S, et al., "On the Failure Condition in Strain-Controlled Low Cycle Fatigue," Intl. J. of Fatigue, No. 3, July 1985, pp. 141-147. 
${ }^{105}$ Bonacuse, P.J., and Kalluri, S., "Axial-Torsional Thermomechanical Fatigue Behavior of Haynes 188 Superalloy," in Thermal Mechanical Fatigue of Aircraft Engine Materials, AGARD-CP-569, 1995, pp. 15 (1-10).

106 Sumner, G., "Heating Methods and Grips," in Techniques for High Temperature Fatigue Testing. G. Sumner and V.B. Livesey, Eds. Elsevier Applied Science Publishers, London, 1983, pp. 71-90

${ }^{107}$ Lappe, V., “Two Color Infrared Thermometers, " Ircon Inc., Niles IL.

108 Jaffar Shah, S., "Field Wiring and Noise Considerations for Analog Signals," National Instruments Application Note 025, 1992.

${ }^{109}$ Loewenstein, E.B., "Reducing the Effects of Noise in a Data Acquisition System by Averaging," National Instruments Application Note 152, 2000.

110 Donald, J.K., "Sampling Rate Effects in Automated Fatigue Crack Growth Rate Testing," in Automation in Fatigue and Fracture: Testing and Analysis, ASTM STP 1231, C. Amzallag, Ed., American Society for Testing and Materials, Philadelphia, 1994, pp. 124-145.

III "Standard Test Method for Young's Modulus, Tangent Modulus, and Chord Modulus", ASTM Designation, E111-82, Section 3, Vol. 03.01, 1993, pp. 222-227.

112 "Standard Practice for Statistical Analysis of Linear or Linearized Stress-Life (S-N) and Strain-Life ( $\varepsilon-N)$ Fatigue Data," ASTM Designation, E739-91, Section 3, Vol. 03.01, 1993, pp. 615-621.

113 Henderson, P.J., Linde, L., "High Temperature Thermo-mechanical and Low Cycle Fatigue of the Oxide Dispersion Strengthened Alloy MA754," in Aspects of High Temperature Deformation and Fracture in Crystalline Materials, Y. Hosoi et al., Eds., JIM, Japan, 1993, pp. 527-534.

114 Scarlin, R.B., "Fatigue Crack Growth in a Cast Ni-Base Alloy," Materials Science and Engineering, Vol. 21, 1975, pp. 139-147. 


\section{Appendix 1}

\section{TMF LabVIEW Testing Software}

The following is a listing of the TMF LabVIEW testing software developed for the IARNRC TMF testing facility. The reader is expected to have some background knowledge of LabVIEW and TMF testing prior to reading this program listing. The LabVIEW User Manual and LabVIEW Data Acquisition Basics Manual (National Instruments part no. 320999B-01 and 320997C-01, respectively) are suggested resources for readers who are not familiar with LabVIEW. Both the main program VI's (Virtual Instruments) and the sub-VI's (subroutines) are listed in this documentation. Virtual instruments pertaining to the control of the MTS 407 controller are not listed in this program listing. The reader should refer to the MTS 407 LabVIEW programming manual (MTS Part No. 151624-00E) for a description of these VI's. Many of the VI's contain multiple frames in the source code listing, only the frames necessary to describe the VI operation are illustrated. For further information on frames not shown in this program listing, the reader should refer to the individual VI block diagrams in the TMF testing software library. 


\section{A1.1 Analog V/O and Digital V/O Signal Connections}

Four analog input channels and are used for data acquisition during testing. Each of these channels has been configured for differential input to mitigate the effects of electrical noise. A summary of the analog input channel configuration used in the TMF testing facility is given as follows:

$\mathrm{AI} \mathrm{Ch}{ }^{*} .0$ : monitors the interlock sense voltage

AI Ch. 1: temperature feedback from the temperature controller

AI Ch. 2: strain feedback from MTS 407 extensometer signal conditioner

AI Ch. 3: load cell feedback from MTS 407 load cell signal conditioner

The temperature and total strain command signals are generated by the data acquisition card. Both analog output channels on the data acquisition card are used for this purpose. A summary of the analog output channel configuration is given as follows:

$\mathrm{AO} \mathrm{Ch.}{ }^{\Psi}$ 0: temperature setpoint command

AO Ch. 1: total strain command

In addition to the using the data acquisition board for analog input and output, two digital channels are used to control two of the relays on the SC2062 digital relay board. One of the relays is used to provide an interlock connection between the data acquisition

\footnotetext{
- AI Ch. - Analog Input Channel

" AO Ch - Analog Output Channel
} 
computer, and the second relay is used for the run/stop connection to the RF generator. The interlock connection between the data acquisition computer and the MTS 407 controller is necessary, since the analog output channels of the data acquisition board by default will go to zero volts when power is removed from ine data acquisition computer. This can cause unexpected actuator movement, possibly damaging the test specimen and mechanical load train components. By default, the digital output channels switch from high-to-low when power is removed from the data acquisition card. Thus, when power is removed from the data acquisition computer, the PC interlock relay will open causing an interlock to trip on the load-unit controller. A summary of the digital channel configuration is as follows:

$\mathrm{DIO}^{\prime} 0$ : run/stop relay for the RF generator; controls relay 6 on the SC 2062 board DIO 1: PC interlock relay; controls relay 7 on the SC 2062 board

Note: In order for the TMF testing software to operate correctly, the AI, AO and DIO channel settings must not be changed from configuration given in this section.

\section{A1.2 TMF Testing Software Theory of Operation}

The testing software is comprised of three major elements as illustrated in the LabVIEW block diagram in Figure A 1.1. The three major elements of the test software are:

1. The program initialization VI's, 
2. The main program body, and

3. Program termination VI's.

The following sub-sections describe the major program elements outlined above.

\begin{abstract}
A1.2.1 Program Initialization
When the TMF test software is run, several front panels open in succession for configuring the software for a test, initializing the data acquisition hardware, and establishing serial communication with the 407 controller. Program initialization is comprised of the following steps. First, the run-relay for the RF generator and PCinterlock relay are closed. Then, serial communication with the MTS 407 controller is initialized. Following this, a panel opens permitting the user to configure test specific settings including, type of test (IT-LCF, OP-TMF or IP-TMF), cycle period, mechanical strain amplitude, temperature range, and data acquisition interval. Then, a panel opens permitting the user to specify the file path and names for data acquisition files. In the final step, the data acquisition board in initialized.
\end{abstract}

\title{
A1.2.2 Main Program Body
}

After the program has been initialized, the relevant test parameters including, the temperature, and mechanical strain waveforms are passed into the main body of the program. The main body of the program is comprised of two loops as illustrated

${ }^{\gamma}$ DIO: Digital Input Output 
schematically in Figure Al.1. The first loop, referred to as the main program loop, is executed once for every cycle, and serves the following purposes:

1. Prompt the user to take actions such as configuring the pyrometer controller for remote setpoint operation, and changing the testing machine to total axial strain-control,

2. Starts and stops the data acquisition at the beginning or end of a cycle,

3. Process that data acquired during one IT-LCF or TMF cycle, and write the data to the data acquisition computer hard disk,

4. Check the tensile load drop criteria by comparing the peak tensile load after one cycle, to a reference value specified by the user, and

5. Determine the next program sequence (case) to execute.

The second loop, referred to as the control loop, is nested within the main program loop. The purpose of the control loop is to generate the temperature and total strain waveforms, and acquire data. This loop is hardware timed, i.e. the hardware clock on the DAQ board controls the data acquisition timing, for accurate timing of the control loop. One temperature and total strain command update occur per iteration of the control loop.

As discussed in Chapter 4 section 4.7, a series of test sequences are followed during TMF and IT-LCF tests. The TMF testing software uses four cases to step through each sequence in the TMF test. Two cases in the test sequence are skipped when conducting an IT-LCF test. Each of these four cases must execute to completion (with the exception of 
isothermal LCF tests), otherwise the program will stop and display an error dialog to indicate the source of the error. The case information is passed through the program during execution via the case manager cluster (see section Al.3.2). The following describe the purpose of each case in the TMF testing software:

Case 0: This case holds the temperature constant for one period of the test cycle. The purpose of this case is to provide the user with sufficient time to adjust the extensometer zero point to compensate for the thermal expansion caused by heating from room temperature. This is accomplished by updating the temperature $\mathrm{AO}$ channel with the first temperature setpoint value contained in the temperature command array (see section A1.3.5). The first element of the temperature command array is either the median test temperature (TMF test) or the isothermal test temperature (isothermal LCF test). When this case has finished executing, the program immediately proceeds to case 1 for TMF tests. For IT-LCF tests, the program will proceed to Case 3. If an error occurred while case 0 is executed the error cluster will be updated, the program will stop executing, and an error dialog box will be displayed to show the source of the error.

Case 1: The purpose of this case is to cycle the temperature (under force control at zero load) for a user specified number of cycles ( $\mathrm{N}-\mathrm{stab})$ to achieve a stable dynamic temperature distribution in the specimen prior to recording the thermal strain compensation data. This is accomplished by updating the 
temperature $\mathrm{AO}$ channel with the contents of the temperature command array, point-by-point, until the control loop has completely cycled through the temperature command array. If an isothermal LCF test is being conducted, this case is not executed.

Case 2: This case cycles the temperature while the recorded thermal strain data commands the servohydraulic-control loop in strain control. The purpose of this case is to assess the accuracy of the recorded thermal strain data prior to applying mechanical strain to the specimen. This is accomplished by updating the temperature and strain AO channels with the contents of the temperature command and thermal strain command arrays point-by-point, until the control loop has completely cycled through the arrays. If an isothermal LCF test is conducted. this case is not executed. This case is repeated for the number of thermal stabilization cycles ( $\mathrm{N}$-stab) before proceeding to case 3.

Case 3: This case cycles the temperature while total strain (sum of thermal and mechanical strain) commands the servohydraulic-control loop in strain control. This is accomplished by updating the temperature and strain AO channels with the contents of the temperature and mechanical strain arrays, point-by-point, until the control loop has completely cycled through the arrays. If an isothermal LCF test is being conducted, the case is executed immediately after case 0 completes execution. This case is repeated until the user interrupts the test, or a user-specified number of cycles has elapsed, or 
when a program error occurs, or the program is stopped by the underpeak detector (see section A 1.5.2.4).

\section{A1.2.3 Program Termination}

If a program error occurs at any point during the execution of the TMF/IT-LCF testing software, or the user terminates the test by pressing the stop button on the user panel, execution of all program code prior to the program termination VI's is aborted. The PC interlock and RF run/stop relays are immediately opened, when program execution is aborted. to de-energize the induction coil and remove hydraulic pressure from the load frame actuator. If an error occurred during program execution an error dialog is displayed.

\section{A 1.3 Definition of Variables}

All program variables (data) passed from the Program Initialization VI's are referred to as clusters in LabVIEW. The following sections explain the data clusters defined for the TMF testing software. Variables that are not listed here can be accessed by using the online help feature within each sub-VI. 


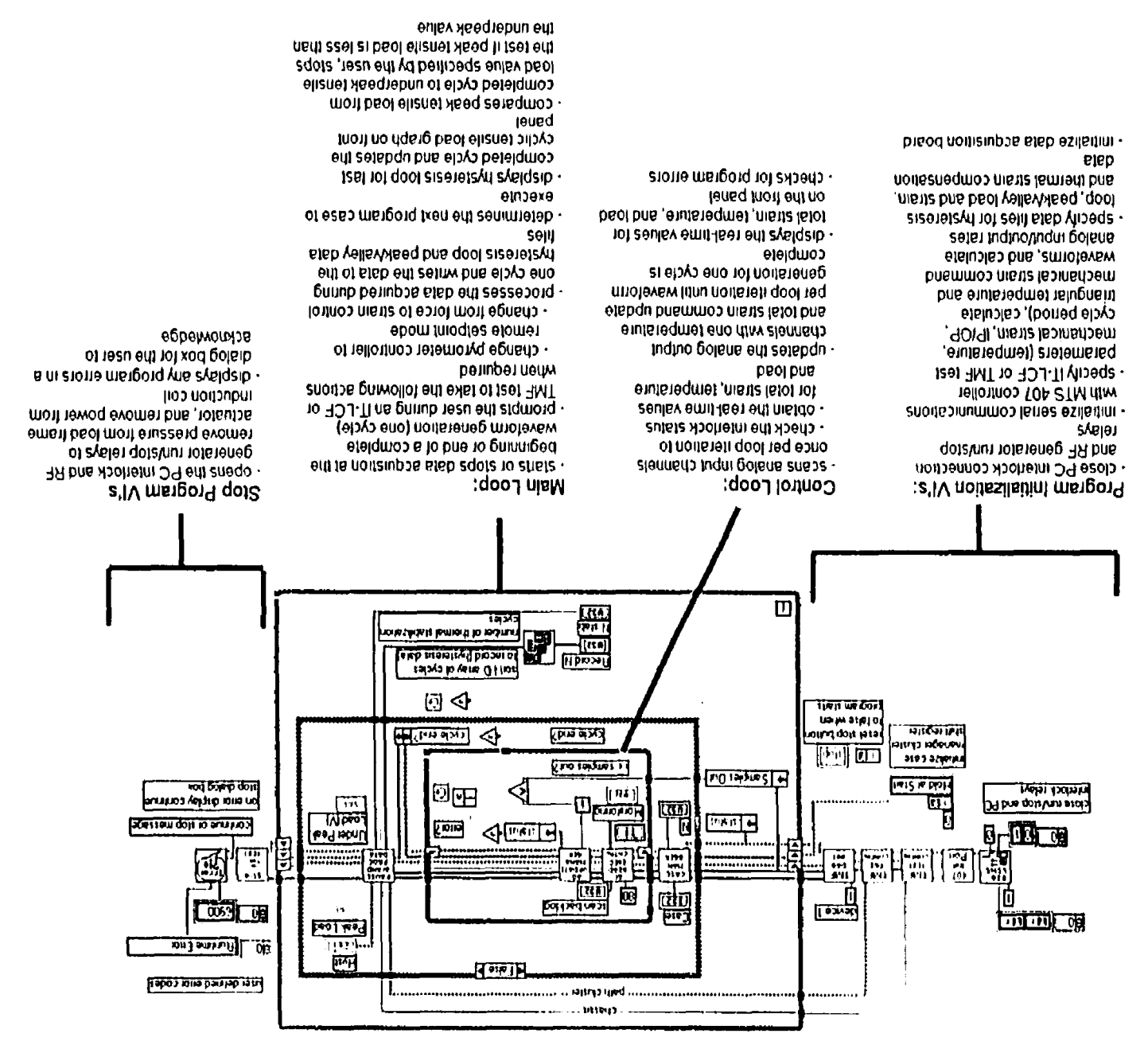




\section{A1.3.1 AVAO taskID Cluster}

The AI/AO taskID cluster contains the task identification numbers used to identify the specific input-output (I/O) operation for the analog input (AI) and analog output (AO) channels of the data acquisition board. Both the AI and AO taskID numbers are 32-bit integer data types that are created when calling AI Config.vi or AO Config.vi. The AV/AO taskID cluster is illustrated in Figure A1. 2. The cluster contents are defined as follows:

- AItaskID - number identifying the analog input I/O operation

- AOtaskm - number identifying the analog output I/O operation

$$
\begin{aligned}
& \text { Al/AOtasklD }
\end{aligned}
$$

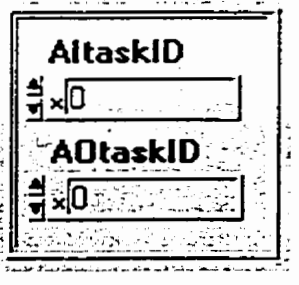

Figure A1. 2 - AI/AO taskID cluster.

\section{A 1.3.2 Case Manager Cluster}

The case manager cluster contains the case number for controlling program execution, the current cycle count $(\mathrm{N})$, and the cycle status (completed or not completed). The case manager cluster is illustrated in Figure A1. 3. The variables contained in the case manager cluster are defined as follows:

- case - program case to be executed 
- cycle end? - cycle status to indicate if all waveform points were generated

- count (N)-in - current cycle count (completed waveform generations)

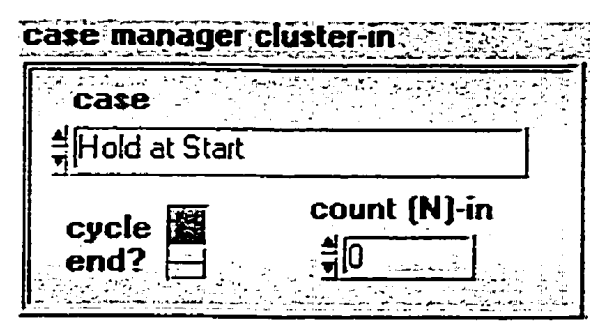

Figure Al. 3 - Case manager cluster.

\section{A 1.3.3 DAQ Parameters Cluster}

The DAQ parameters cluster contains six variables which are used to specify the DAQ initialization parameters, temperature-strain phasing, number of waveform points (samples), data logging interval (time between samples), and number of cycles used to define run-out life. The DAQ parameters cluster is illustrated in Figure Al. 4 and the cluster contents are defined as follows:

- Samples-Out - number of triangular waveform samples

- Phasing-Out - phase of temperature and strain

- scan rate - analog input scan rate [scans/second]

- data log interval-out - interval to write data to file [seconds]

- update rate - analog output update rate [updates/second]

- Number of cycles, $\mathbf{N}$ - test run out (test stop condition) [cycles] 


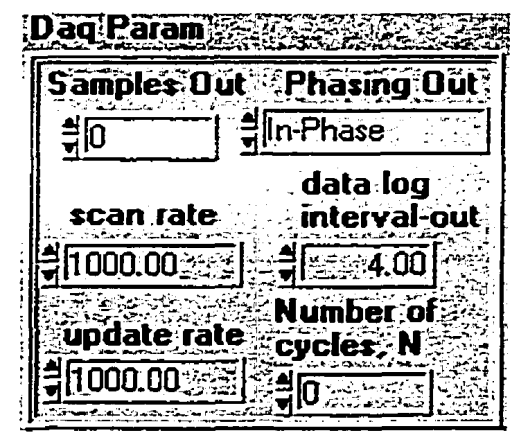

Figure Al. 4-DAQ Parameters cluster.

\section{A 1.3.4 Error-in Cluster}

The error-in cluster contains a Boolean that indicates whether an error has occurred, the code for the error, and the source or name of the VI that returned the error. The error in cluster is illustrated in Figure A 1.5. The variables in the error-in cluster are defined as follows:

- $\quad$ status - error status (Boolean T/F)

- code - code defining the error if status is true (default is 0 - no error)

- source - the error source (usually VI name where error occurred)

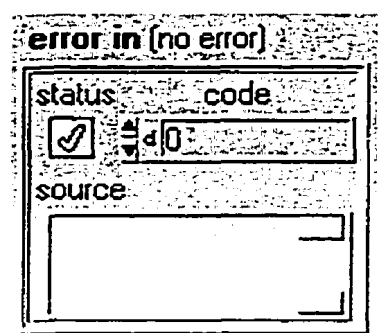

Figure AI. 5-Error-in cluster. 


\section{A1.3.5 Waveform Cluster}

The waveform cluster contains three arrays defining the mechanical strain, temperature and thermal strain waveforms in volts. The waveform cluster is illustrated in Figure A1.6. The cluster variables are defined as follows:

- Mech Strain (V) - array defining the mechanical strain waveform

- Temperature (V) - array defining the temperature waveform

- Thermal Strain (V) - array defining the thermal strain waveform

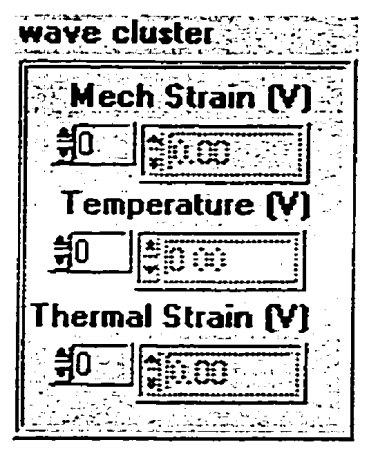

Figure A1. 6- Waveform cluster.

\section{A1.4 Program Initialization VI's}

The configuration and initialization VI's are described in the following sections. These VI's serve the purpose of allowing the user to specify test parameters, initialize the data acquisition board, and establish serial communication with the MTS 407 controller. The VI's are described in the order that they are called in the TMF testing application. SubVI's for program initialization are also documented. 


\section{A 1.4.1 407 init port.vi}

This VI is used to initialize the MTS 407 controller for serial communication. This VI will loop until the 407 controller has been initialized correctly, and the 'done' button is pressed by the user. The 'port error LED will light up 407 init port.vi front panel if the serial port could not be initialized. If a serial connection to the 407 controller is established, the 'initialized' LED will light up on the 407 init port.vi front panel and the 'done' button will be visible to the user. The 407 controller is also initialized when this VI is executed.

This VI must be called once for every LabVIEW session that will access the 407 controller. If the port number, baud rate and timeout value are always constant, this port initialization may be done without operator intervention, but the global element must be loaded. The connector pane and block diagram for this sub-VI are illustrated in Figure A1. 7 and Figure A1. 8, respectively.

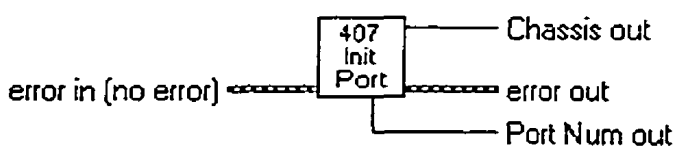

Figure A1. 7 - Connector pane for 407 init port.vi. 

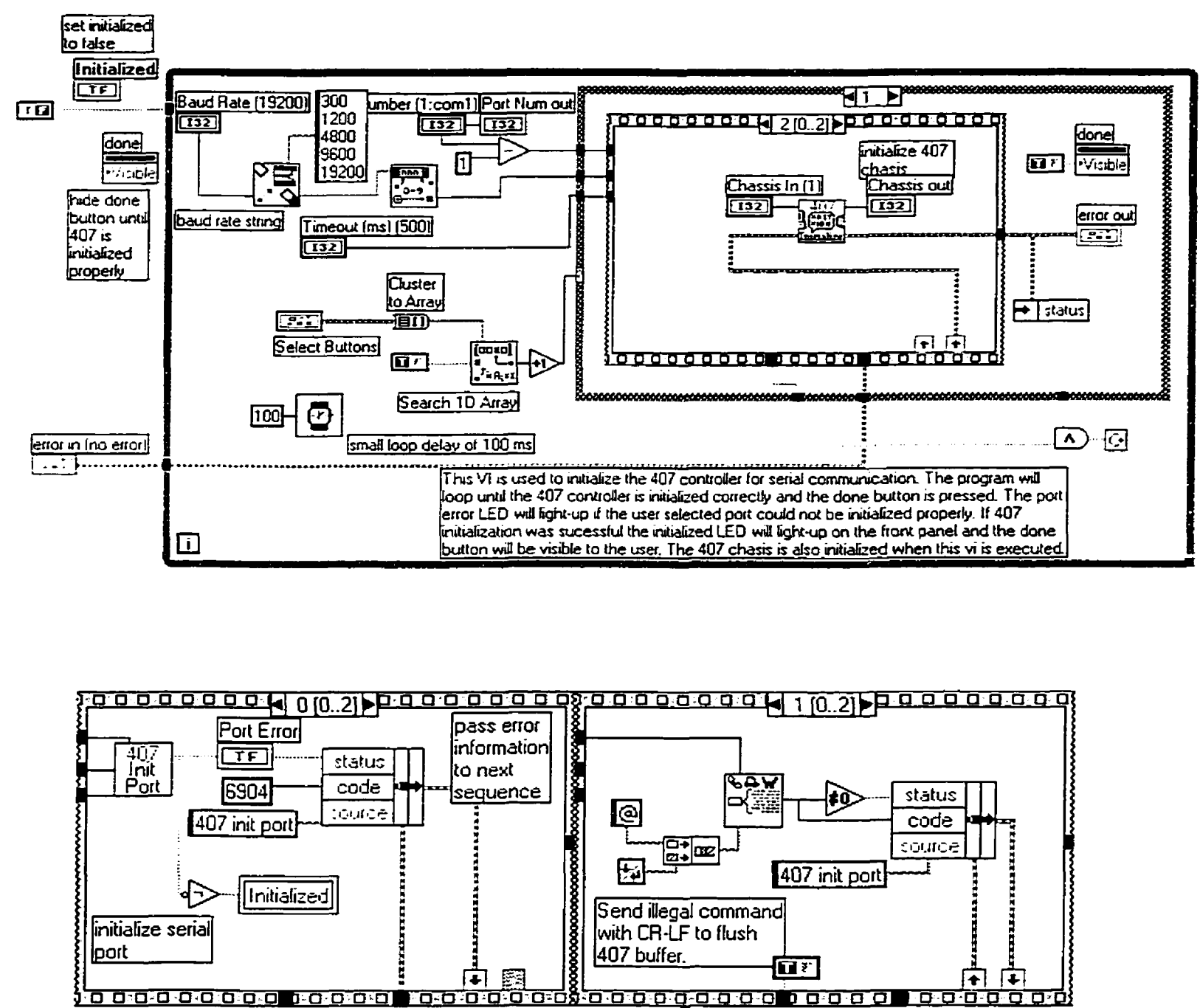

Figure A 1. 8 - Block diagram illustrating selected frames from 407 init port.vi.

\section{A1.4.2 TMF config.vi}

This VI allows the user to specify the settings for a TMF or isothermal LCF test. Initially when this VI is run, all user configurable settings are hidden on the front panel. To access a specific set of test parameters, the user must select the item to configure from the drop down menu and press the 'adjust' button. To change another test parameter, the user must press the 'next' button at the bottom of the front panel. This VI continues to loop until 
the user has pressed the 'done' button signifying that the test configuration is complete. The user can adjust the follow test parameters:

1. Test type (in-phase, out-of-phase, isothermal LCF), cycle period and test duration in cycles,

2. Data acquisition interval,

3. Mechanical strain span (amplitude), and

4. Temperature setpoint (median value) and span (amplitude).

The user is cautioned to check settings carefully, since minimal error checking is provided to prevent out of range strain and temperature values. The values specified by the user are used to calculate the temperature, and mechanical strain arrays (in volts), which are used to control the test. If an error is detected from a previous VI, the error information is passed onto the next VI without allowing the user to configure the test. The connector pane and block diagrams are shown in Figure Al. 9 and Figure A1. 10, respectively.

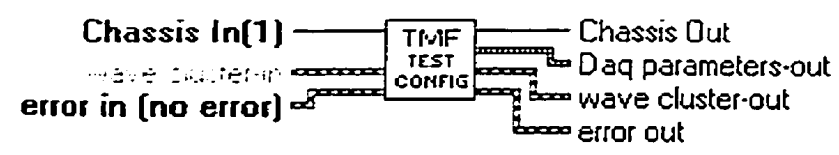

Figure A 1. 9 - TMF test config.vi connector pane. 

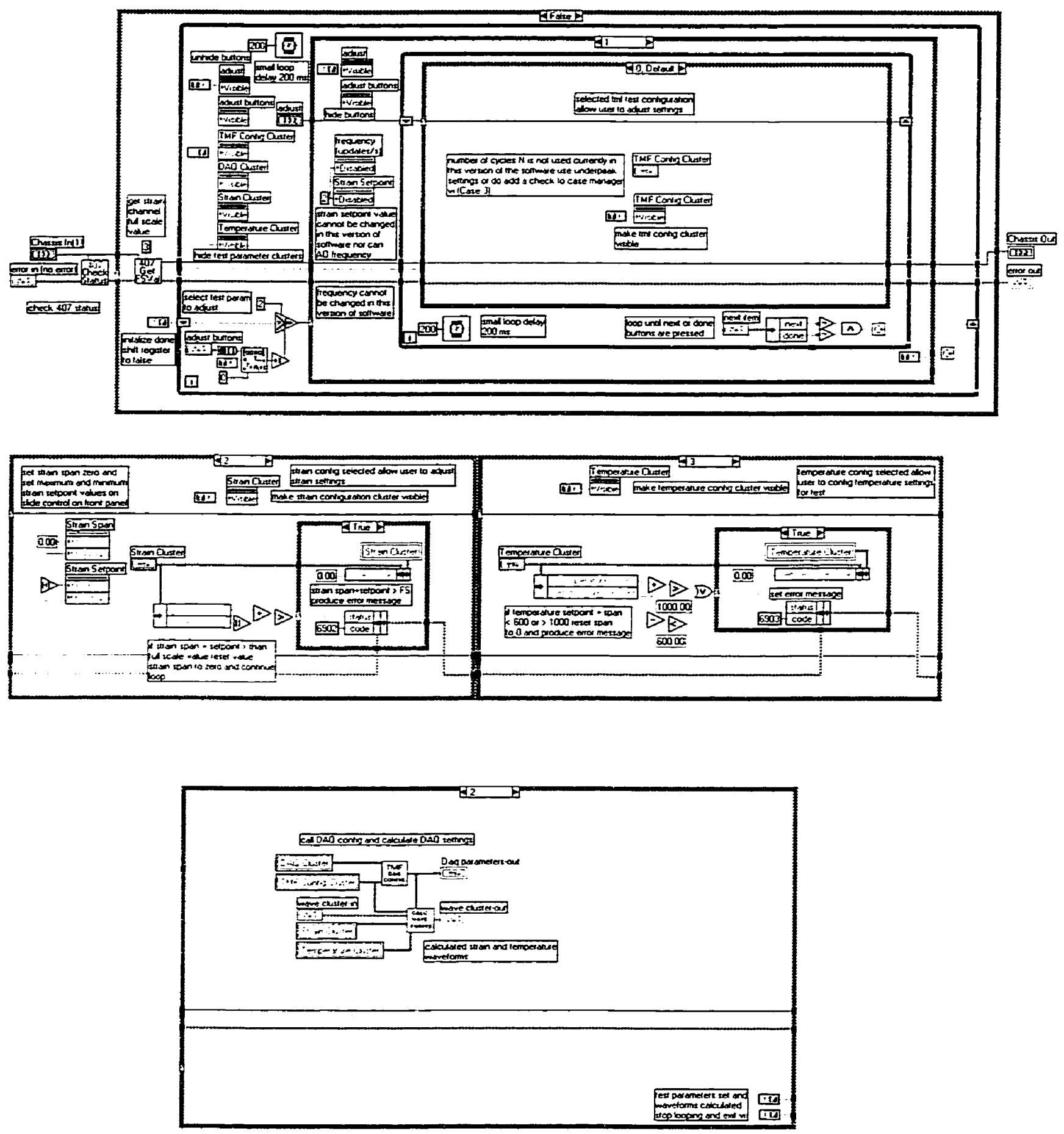

Figure A I. 10-Block diagram illustrating selected frames from TMF config.vi. 


\section{A1.4.2.1 Daq config.vi}

This VI calculates the DAQ parameters such as $\mathrm{AI}$ scan rate and $\mathrm{AO}$ update rate for a TMF test. A minimum of 4000 samples (points) is used to generate the waveforms to obtain approximately 12-bit precision. If the TMF cycle period is less than 4000 samples divided by the AO update rate, the number of waveform samples is set to 4000 , and the Al scan rate and $\mathrm{AO}$ update rate are changed accordingly. The AI update rate is multiplied by 80 to give an eighty times oversampling rate so that $80 \mathrm{AI}$ data points are averaged per $\mathrm{AI}$ scan. Presemtly the $\mathrm{AO}$ update rate is locked at 20 updates per second (one update every $50 \mathrm{~ms}$ ). The VI connector pane and block diagram are illustrated in Figure Al. 11 and Figure Al - 12, respectively.

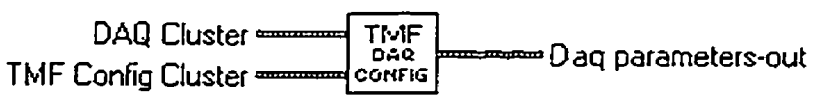

Figure Al. 11 - Connector pane for daq config.vi.

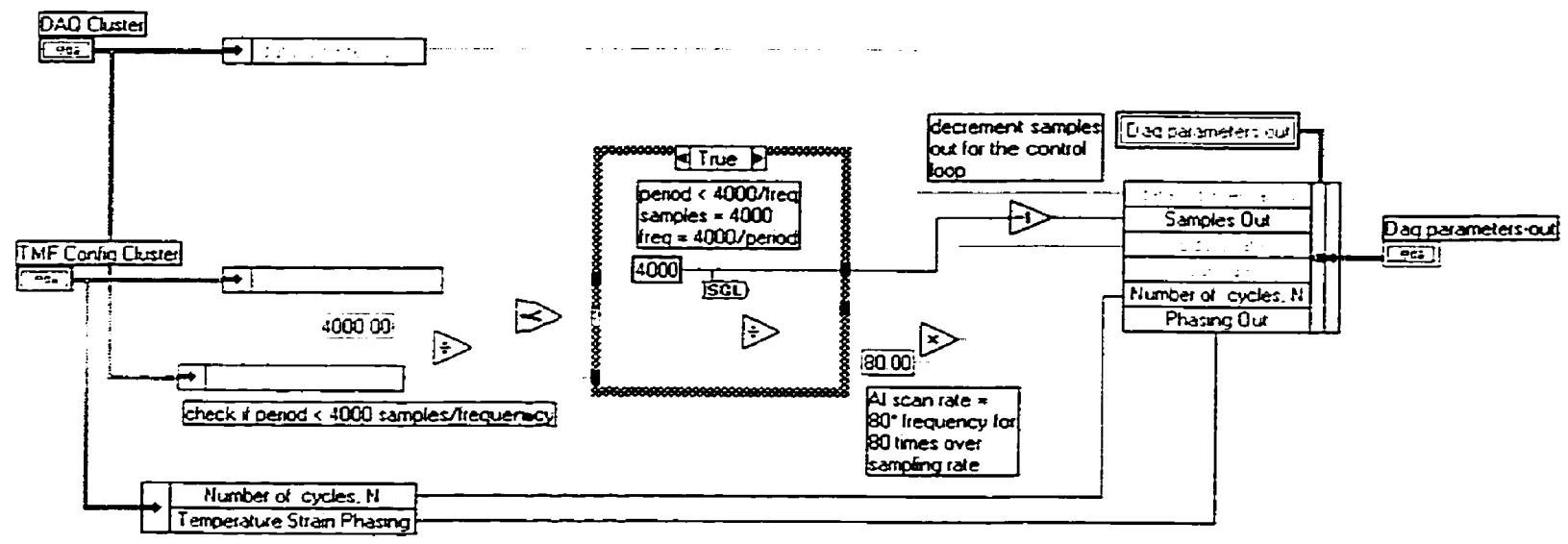

Figure Al. 12 - Block diagrarn for daq config.vi. 


\section{A1.4.2.2 Calculate wa veforms.vi}

This VI calculates the temperature and mechanical strain waveforms (in volts) based on the setpoint and span values specified by the user. The mechanical strain waveform, calculated by Compute Wave.vi (see section A1.4.2.2.1), is phase-shifted 270 degrees to start the test in compression. Based on the type of TMF test (IP or OP), the phase of the temperature setpoint waveform is altered to obtain the desired temperature-strain relationship. For example, the temperature waveform phase is shifted by 90 degrees for an out-of-phase so that the temperature is increasing as the strain value is decreasing. Conversely, the temperature waveform phase is shifted by 270 degrees for an in-phase so that the temperature is decreasing as the strain value is decreasing. For isothermal-LCF tests. the temperature waveform phase is irrelevant, and therefore it is set to zero degrees. The temperature range of the Ircon temperature controller used in the present study is $600-1300^{\circ} \mathrm{C}$. Therefore, the 'full scale' temperature span is given by the difference between two temperature extremes of the temperature controller $\left(1300-600=700^{\circ} \mathrm{C}\right)$. The temperature span value specified by the user is converted to volts based on the full scale temperature span.

The temperature setpoint is converted from engineering units $\left({ }^{\circ} \mathrm{C}\right)$ to volts and is used to offset the temperature waveform so that the proper temperature waveform is attained. The number of waveform samples in the temperature waveform is dictated by the DAQ config.vi, which calculates this value. The VI connector pane and block diagram are illustrated in Figure AI. 13 and Figure A1. 14, respectively. 


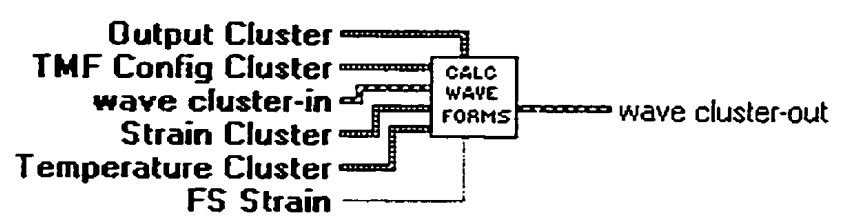

Figure Al. 13 -Connector pane for calculate waveforms.vi

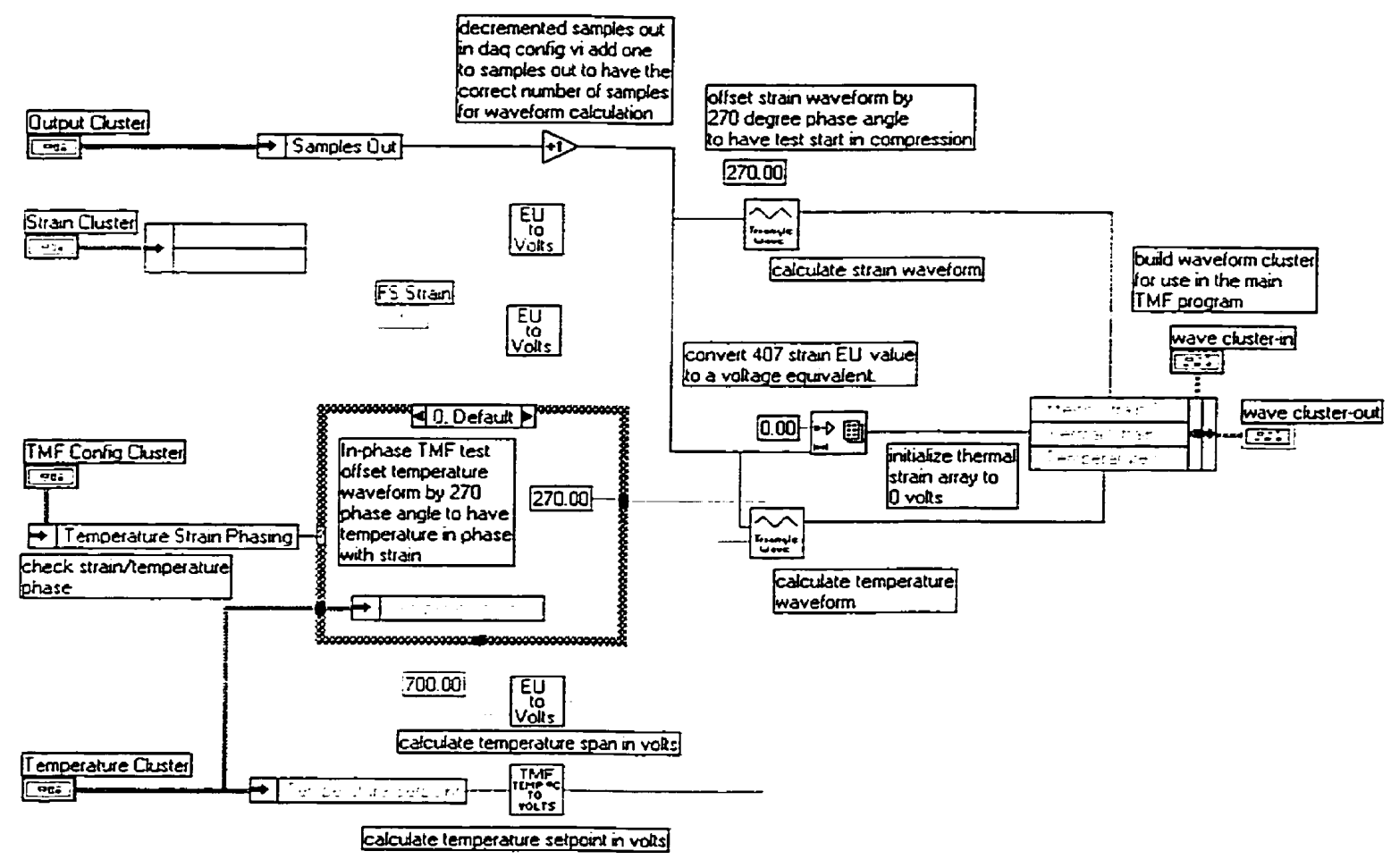

Figure A 1. 14 - Block diagram for calculate waveforms.vi.

Note: TMF and IT-LCF tests are typically conducted under fully reversed conditions with zero mean strain. Therefore, waveforms.vi has not been programmed to accept a non-zero mean strain. If test requirements require a non-zero mean value for strain, a ramp-up procedure can be added to the program to accommodate this. 


\section{A1.4.2.2.1. Compute wave.vi}

This VI computes a triangular waveform based the following input values:

1. Number of points - number of points in the triangular waveform

2. Phase - the phase angle of the triangular waveform

3. Amplitude - the amplitude of the triangular waveform

4. Offset - the offset (mean value) of the triangular waveform.

The connector pane and block diagram are illustrated in Figure Al. 15 and Figure A1. 16, respectively.

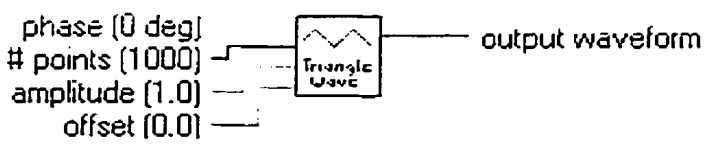

Figure Al. 15 - Connector pane for compute wave.vi.

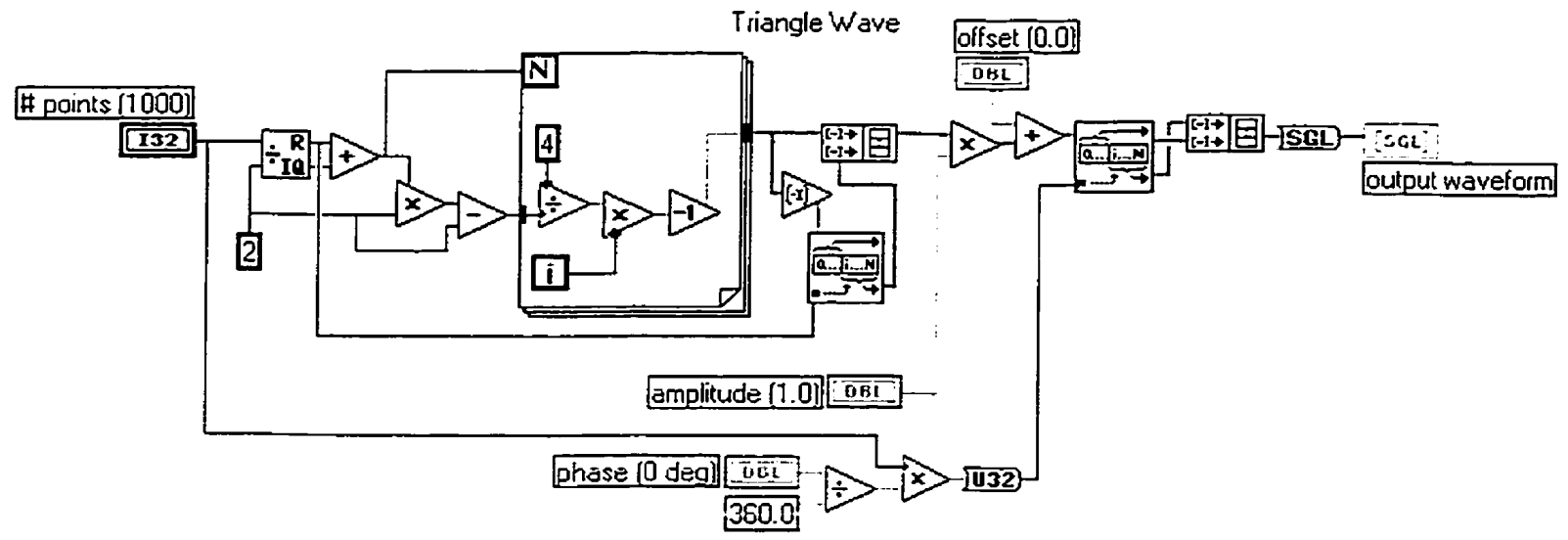

Figure Al. 16-Block diagram for compute wave.vi. 


\section{A1.4.2.2.2. Ircon Modline Plus T to V.vi}

This VI is used to convert a temperature in degrees Celsius to an analog voltage that is proportional to the setpoint of the Ircon Modline Plus infrared pyrometer. The calibration constants of this VI need to be changed whenever the temperature setpoint analog input is calibrated. The connector pane and block diagram are illustrated in Figure Al. 17 and Figure A1. 18, respectively.

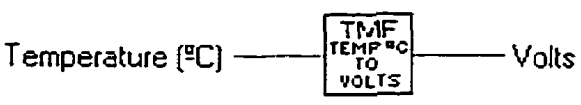

Figure A 1. 17 - Connector pane for Ircon Modline Plus T to V.vi.

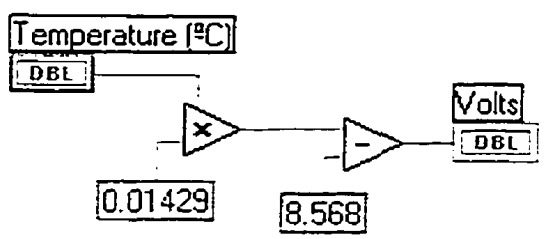

Set point voltage $=0.07429^{\times}$tempurature -8.568

Figure Al. 18 - Block diagram for Ircon Modline Plus T to V.vi.

\section{A 1.4.3 TMF file config.vi}

This VI allows the user to specify the path and filenames for the hysteresis loop (datalog file), peak/valley load and strain ( $\mathrm{pk} / \mathrm{vl}$ load and strain), and thermal strain data files. If an IT-LCF test is conducted, the thermal strain the thermal strain data file is not created. This VI loops until the user has input all filenames and paths, and presses the 'done' button. If the user presses the 'done' button without specifying valid paths for the data files, the user will be prompted to specify the path and name for each data file. After the 
'done' button is pressed the files are created. A file header containing the date and specimen number is appended to the beginning of each data file. If an error has occurred in a previous VI, this VI simply passes the error information through without creating any datalog files. The connector pane and block diagram for TMF file config.vi are illustrated in Figure AI. 19 and Figure A1. 20, respectively.

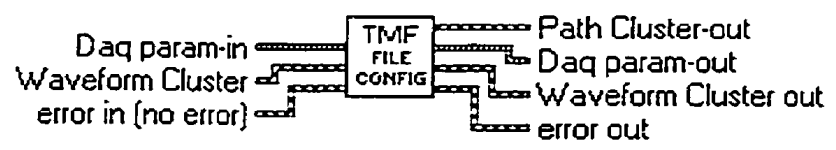

Figure A I. 19 - Connector pane for TMF file config.vi.

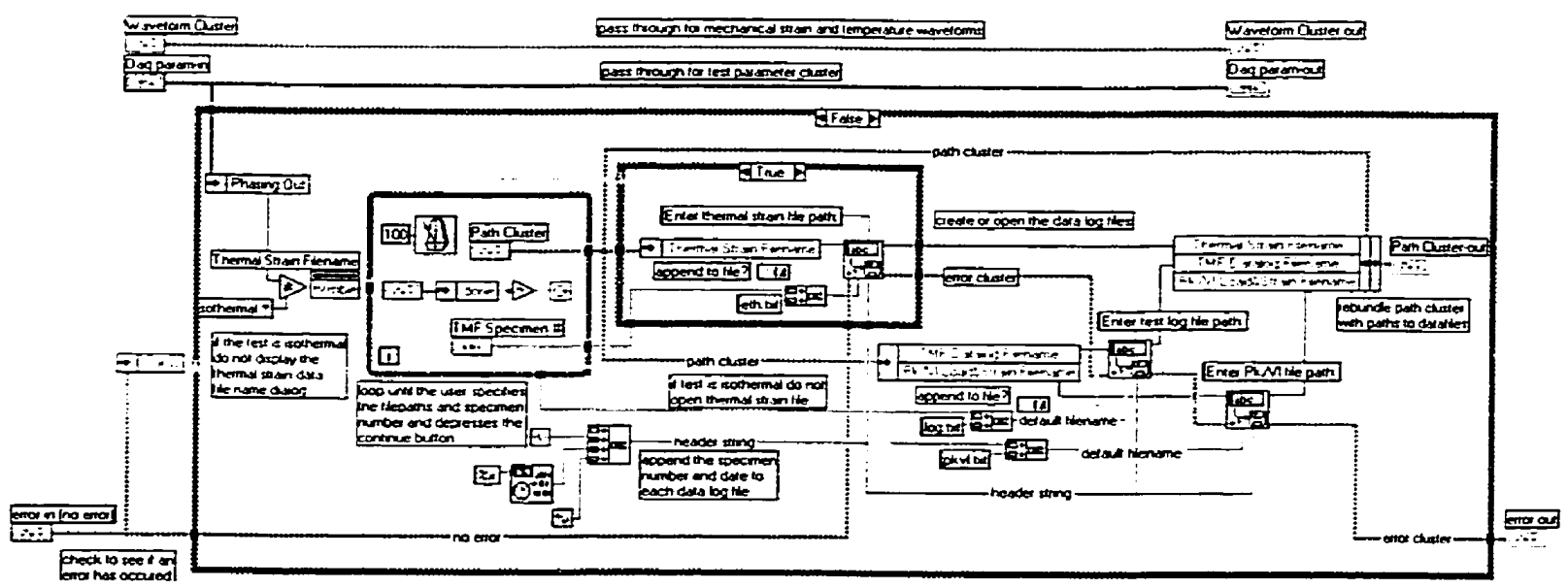

Figure A1. 20 - Block diagram for TMF file config.vi.

\section{A1.4.4 Daq card initialize.vi}

This VI initializes the data acquisition (DAQ) board by setting up the AI and AO buffers, AI scan rate, and digital triggers. Digital triggering is not used in the TMF software but has been provided here as a convenience. The device number for the DAQ board used in 
the TMF facility is one (1). If the NI-DAQ configuration is changed such that any other device number is used instead of one (1), this value must be changed to ensure the DAQ card initializes properly. The connector block and block diagram are illustrated in Figure A1. 21 and Figure A1.22, respectively.

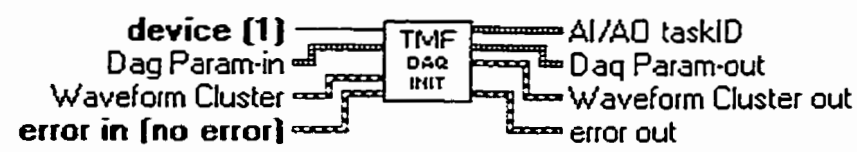

Figure Al. 21 - Connector pane for daq card initialize.vi.

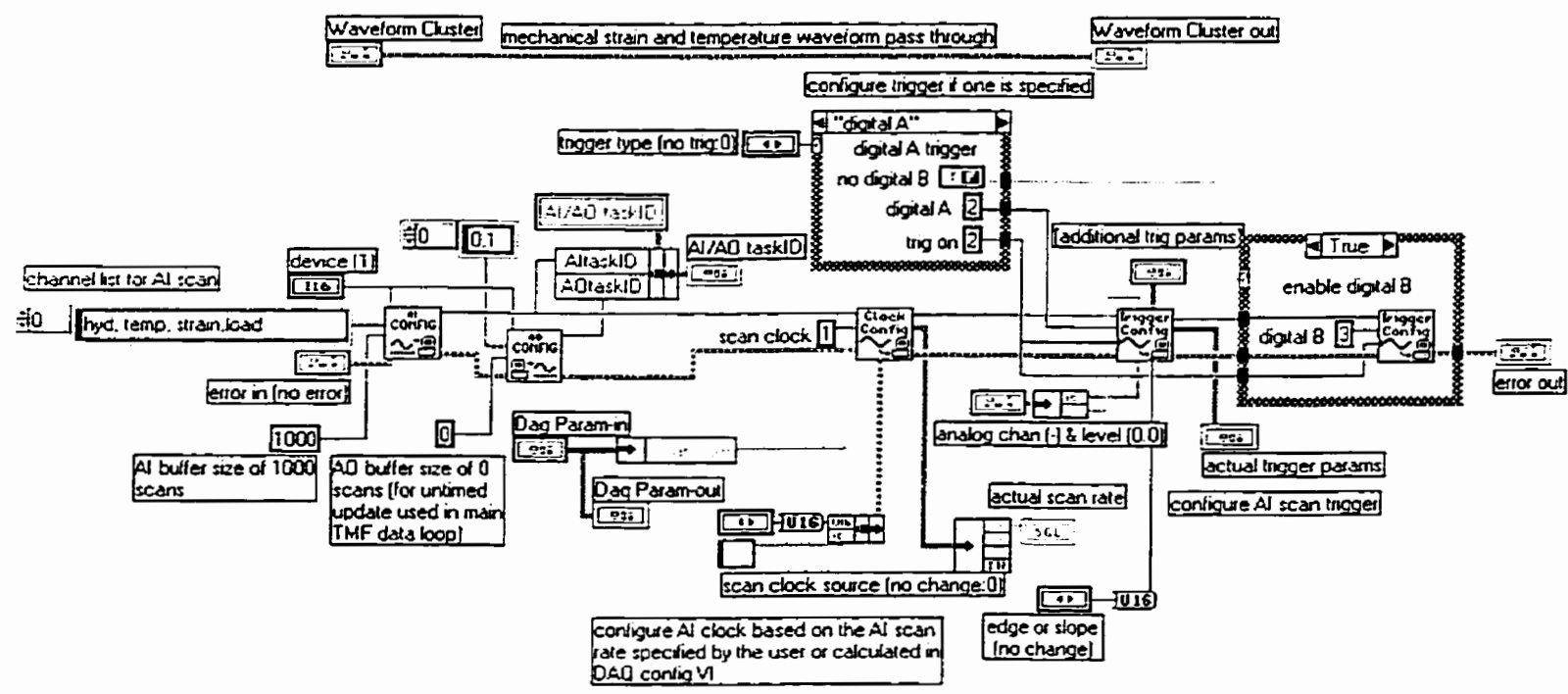

Figure Al. 22 - Block diagram for daq card initialize.vi.

Note: The AI channel list shown in Figure A1.22 indicates that the AI channels have been named to correspond to the purpose of the channel. This implies that these channel names must be configured in the NI-DAQ configuration file. If any of these channel names are changed in the NI-DAQ configuration file, then they must be updated here to prevent an error from occurring during DAQ card initialization. 


\section{A1.5 Main Program Body VI's}

The TMF testing application (program) main program body VI's are described in the following sections. The main program body VI's are used to control the test sequence during the test, interactively prompt the user to take certain actions during a test, and start or stop data acquisition at the be:ginning and end of a cycle, respectively. These VI's also update the user interface (e.g. up.date the hysteresis plot on the TMF test application front panel after a cycle has completedd), determine the maximum/minimum mechanical strain and load during a cycle, and write the data acquired during the cycle to data files specified by the user. Sub-VI's fror the program control and data processing VI's are also presented.

\section{A1.5.1 Case manager.vi}

This VI reads the input case form the case manager cluster and will interactively prompt the user to take certain actions. Actions include, prompting the user to switch the temperature controller to external command, and changing the test controller from force to strain control. This VI also starts the analog input data acquisition, and determines the relative time value used for computing the elapsed time during a cycle. A series of cases are used to determine which actions will be taken by this VI:

Case 0: The first temperature setpoint value stored in the temperature array is output to the temperature comtrol AO channel. The strain command AO channel is updated with zero volts. The user is prompted to switch the temperature controller to external setpoint control and confirm that they have taken this action by pressing the 'OK' or 'Cancel' button. 
Case 1: No user intervention is required for Case 1 , therefore, all values are passed through the Case Manger.vi without changes.

Case 2: If the current cycle count is zero, then the first temperature value stored in the temperature array and the first thermal strain command stored in the thermal strain array are output to the two analog output channels, otherwise no action is taken. Then the user is prompted to switch the test controller from force to strain-control. If the user selects the ' $\mathrm{OK}$ ' button, the test is started. If the Cancel' button is pressed, the error cluster is updated to indicate the user has canceled the test.

Case 3: If an isothermal LCF test is being conducted, and the current cycle count is zero. the first value in the temperature array will be output to the temperature control channel, and the strain control channel is updated with zero volts. It is important to realize that by commanding zero volts on the strain control channel that the extensometer must be zeroed before changing the control mode. The user is prompted to switch to strain control and press 'OK' to start the test. If a TMF test is conducted, this case will not take any action.

The connector pane and block diagram for case manager.vi are illustrated in Figure AI. 23 and Figure A1. 24, respectively. 


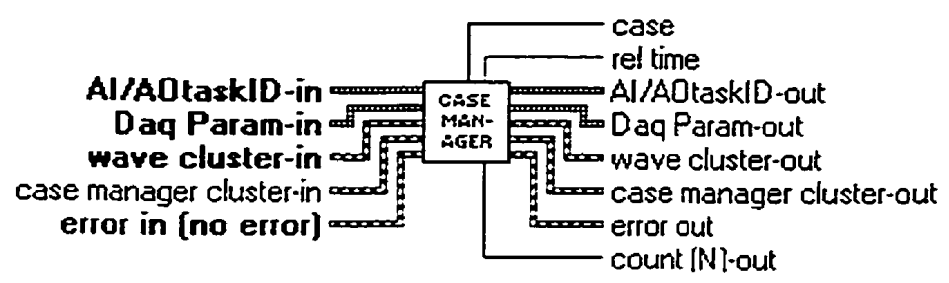

Figure Al. 23 - Connector pane for case manager.vi.
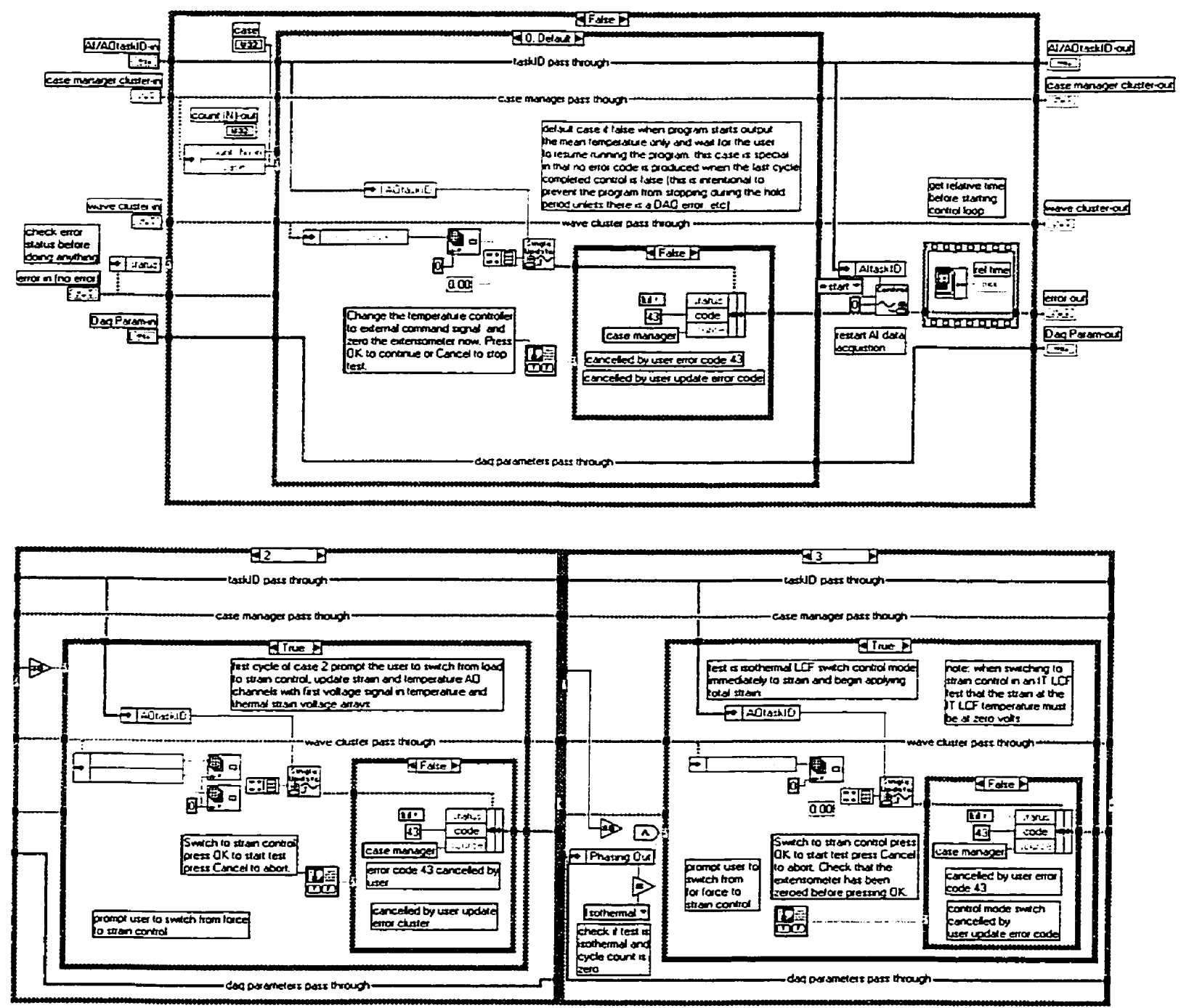

Figure Al. 24 - Block diagram for case manager.vi. 


\section{A1.5.2 Pause AI and process.vi}

This VI pauses the data acquisition, processes test data and controls the execution of the program. It is assumed that the user has set the number of thermal stabilization cycles $(\mathrm{N}$ stab) to a value greater than 1 or else this VI will not work correctly. The $\mathrm{N}$ stab control on the main TMF program front panel limits the range of values such that values less than $\mathrm{I}$ are coerced to a valid value (i.e., a value greater than 1 ). The following pseudo code explains the operation of this VI:

pause data acquistion immediately check current error status

IF error code is between $I$ and 85 THEN ELSE do not process data and pass error to next vi

go to the case in case manager cluster

IF last cycle completed THEN

ELSE increment count

update error code to 6900 (Runtime Error)

Case 0:

reset cycle counter to zero

IF test is isothermal THEN change case to cycle temperature and total strain (case 3)

ELSE

change case to cycle temperature for $\mathrm{N}$ stab cycles (case 1 ) ENDIF

\section{Case 1:}

IF cycle count greater than the number of stabilization cycle ( $\mathrm{N}$ stab) - 1 THEN change case to cycle temperature and thermal strain (case 2) reset cycle counter to zero process thermal strain data and write to thermal strain data file 


\section{ELSE \\ continue with present case (case I) \\ ENDIF}

Case 2:

IF cycle count greater than the number of stabilization cycles ( $N$ stab) - 1 THEN reset cycle counter to zero

ELSE change case to cycle temperature and total strain (case 3 )

\section{ENDIF}

continue with present case (case 2)

\section{Case 3:}

process and write peak/valley load and strain values to file process hysteresis loop data continue with present case

check if peak load is less than the under peak load specified by the user

build an array of all error codes and find the first error that occurred build a string of all error sources and select error source if code $!=0$ re-bundle next case to execute on next loop

\section{ENDIF}

The connector pane and block diagram for pause AI and process.vi are illustrated in

Figure A I. 25 and Figure A I. 26, respectively.

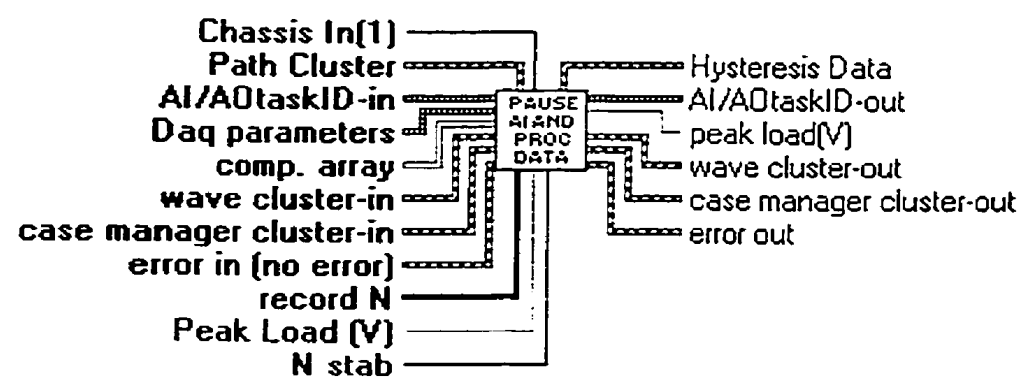

Figure Al. 25 - Connector pane for pause AI and process.vi. 

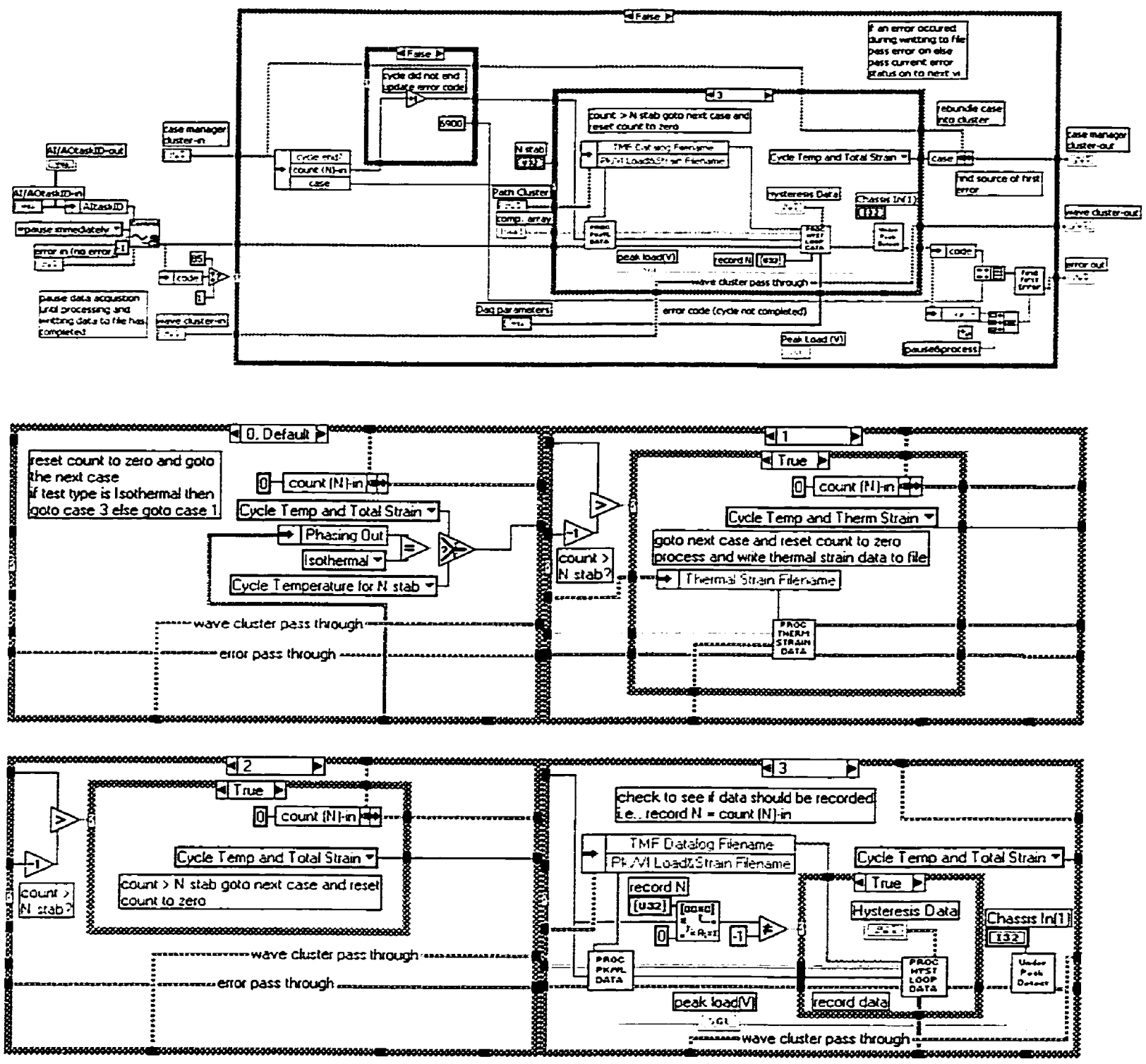

Figure A1. 26 - Block diagram for pause AI and process .vi.

\section{A 1.5.2.1 Process ther mal strain data.vi}

This VI takes the composite command and feedback signal array (comp. array) and separates the time, temperature, and thermal strain feedback signals (in volts). These three items are combined into an array and appended to the thermal strain data file. The waveform cluster (wave cluster) is updated with the thermal strain data and adds the 
thermal strain and mechanical strain signals together to give the total strain command signal used to drive the servo-loop. This VI only checks for error codes in the range of 1 to 85 and will pass the error information to the next VI without processing data if an error code is in this range. The connector pane and block diagram for process thermal strain data.vi are illustrated in Figure A1. 27 and Figure A1. 28, respectively.

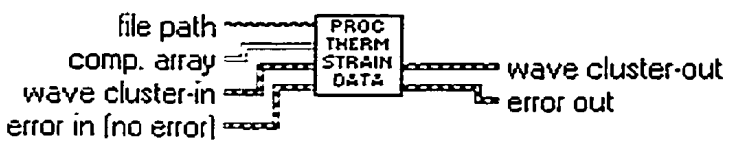

Figure Al. 27 - Connector pane for process thermal strain data.vi.

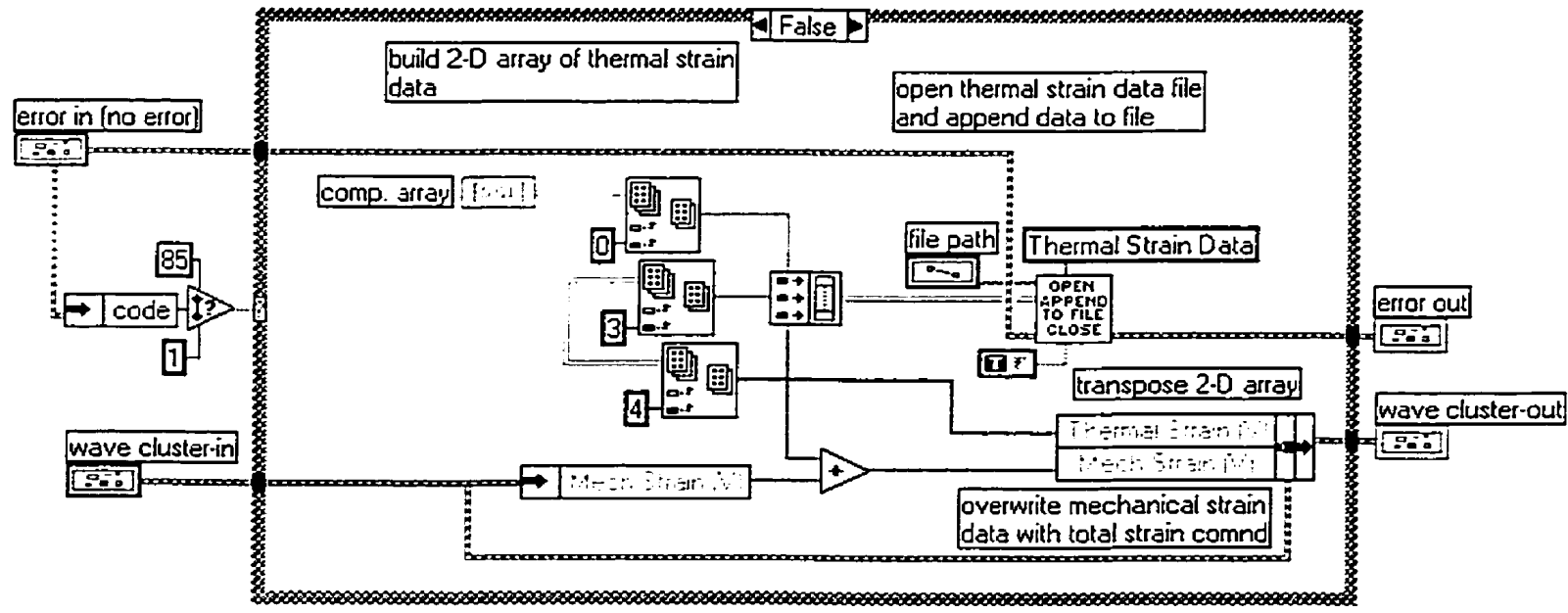

Figure A I. 28 - Block diagram for process thermal strain data.vi.

\section{A1.5.2.1.1. Open and append to file.vi}

This VI opens the data file specified in file path. appends a formatted string of data to the file, and then closes the data file. The incoming error code is checked; if the error code is in the range $\mathrm{I}$ to 85 , no action is taken other than passing the error information to the next 
VI. If the error code is not in the afore mentioned range, the VI will build a spreadsheet string from any 2-D array of single precision data passed to this VI. Depending on what is being writing to the data file several different actions can be taken (several different cases).

Case 0: The data being passed to this VI is thermal strain data; the spreadsheet string is immediately written to the file.

Case I: The data being passed to thīs VI is peak/valley load and strain data, append the current cycle number to the spreadsheet string then write the string to the data file.

Case 2: The data being passed to this VI is hysteresis loop data, append the ampersand character and current cycle count to the spreadsheet string then write the string to the data file.

The connector pane and block diagram are illustrated in Figure AI. 29 and Figure A1. 30, respectively

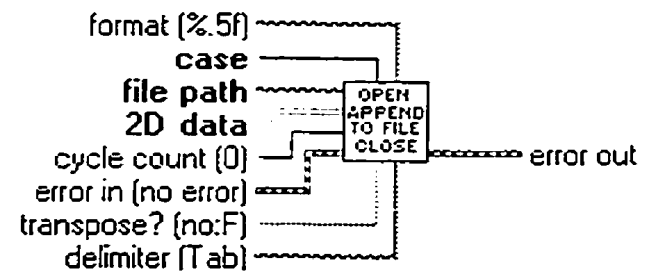

Figure A1. 29 - Connector pane for open and append to file.vi. 


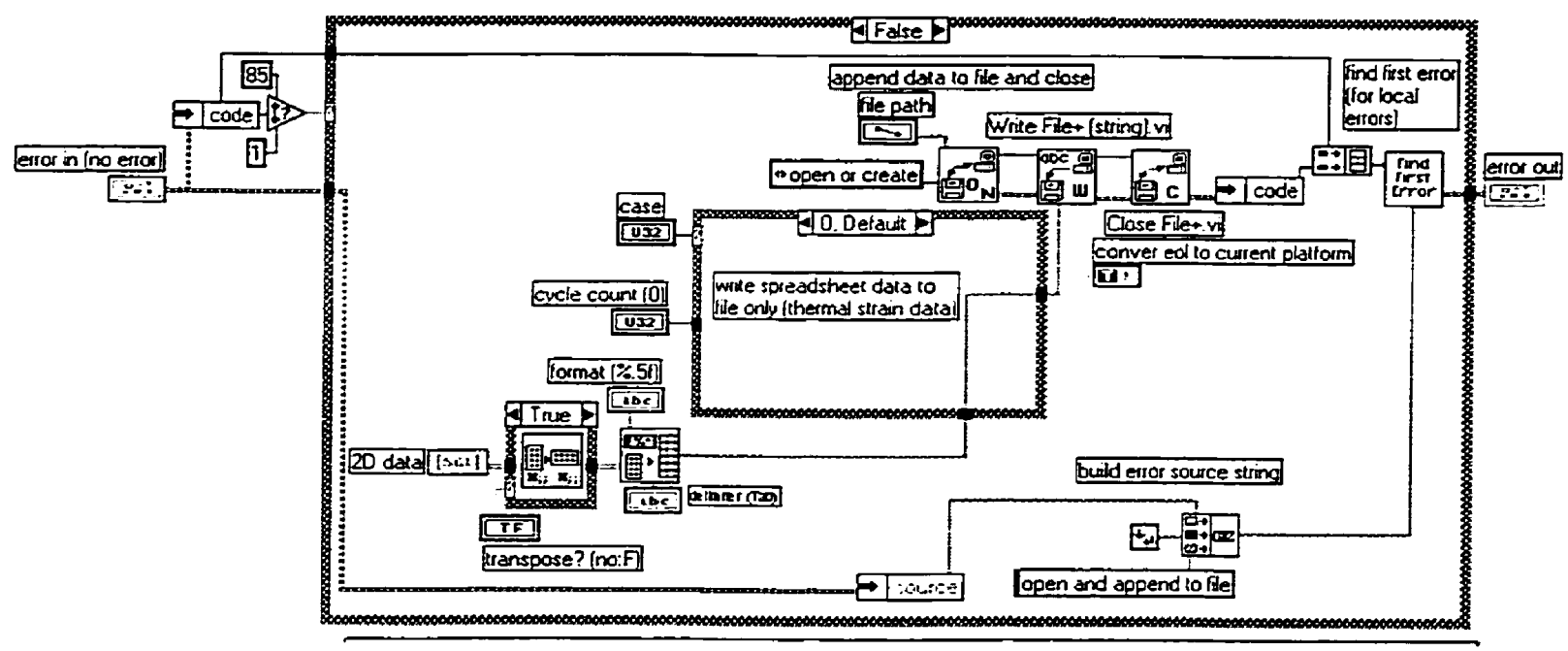

Figure A1. 30 - Block diagram for open and append to file.vi.

\section{A1.5.2.2 Process peak valley data.vi}

This VI processes the composite array (array of command and AI feedback signals) to give the maximum and minimum total strains, mechanical strains, and loads during a given test cycle. This VI only checks for error codes in the range of 1 to 85 and will pass the error information to the next VI without processing the data if an error code is in this range. If an error is encounter when writing the maximum and minimum (peak valley) values to the peak/ valley data file, the error cluster will be updated. Note: if the AI channel configuration is changed, this VI must be updated to pick the correct channels to process. The connector pane and block diagram for process peak valley data.vi are illustrated in Figure A1. 31 and Figure A1. 32, respectively.

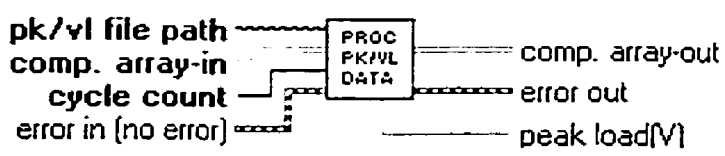

Figure Al. 31 - Connector pane for process peak valley data.vi. 


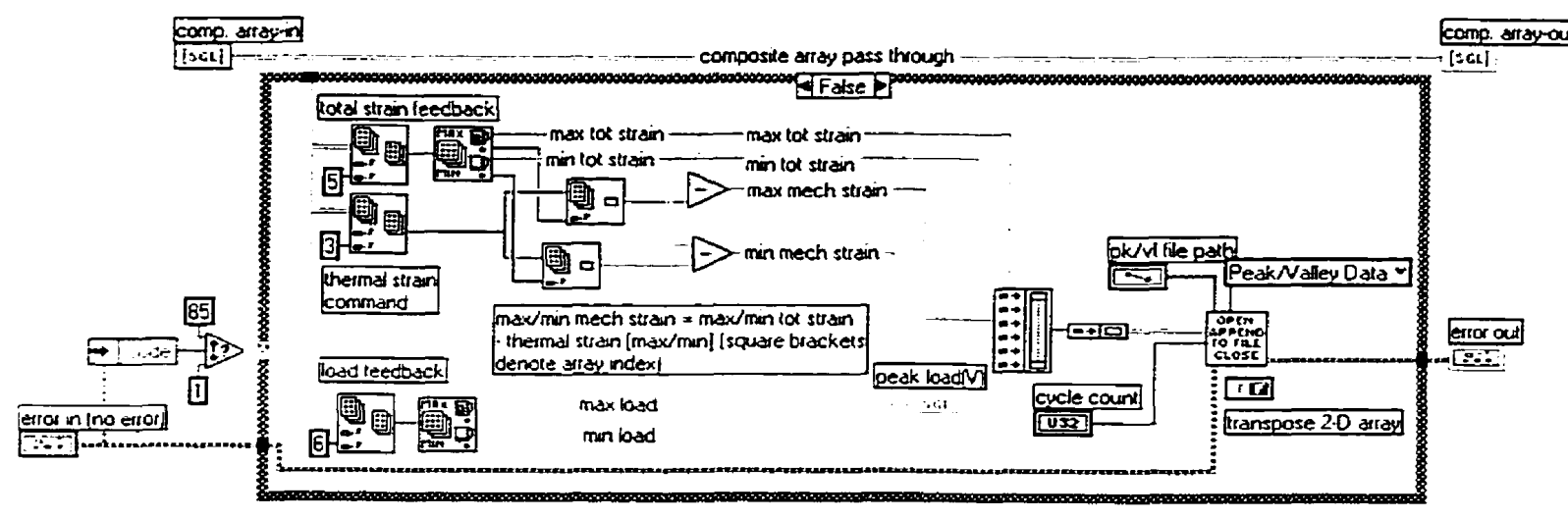

Figure A1. 32 - Block diagram for process peak valley data.vi.

\section{A1.5.2.3 Process hyst eresis data.vi}

After a cycle is completed, this VI takes the resulting composite array (array of command and AI feedback signals) decimates the array, updates the hysteresis loop plot on the TMF software display panel. and writes the hysteresis loop data to the DAQ computer hard disk if the record cycle array contains an element equal to the current cycle count. If an error is encounter while writing the hysteresis loop data, the error information will be passed through this VI. Note: this VI destroys the composite array during the decimation process, and therefore should be called last to ensure the integrity of the composite array for other VI's. This VI only checks for error codes in the range of 1 to 85 and will pass the error information to the next VI without processing data, if an error code is in this range. The connector pane and block diagram for this VI are illustrated in Figure A1. 33 and Figure A1. 34, respectively. 


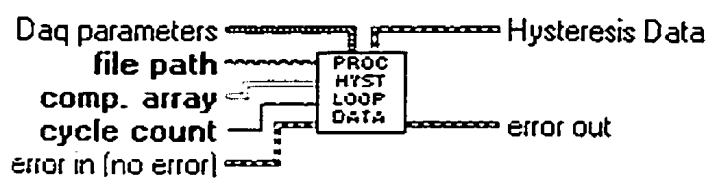

Figure Al. 33 - Connector pane for process hysteresis data.vi.

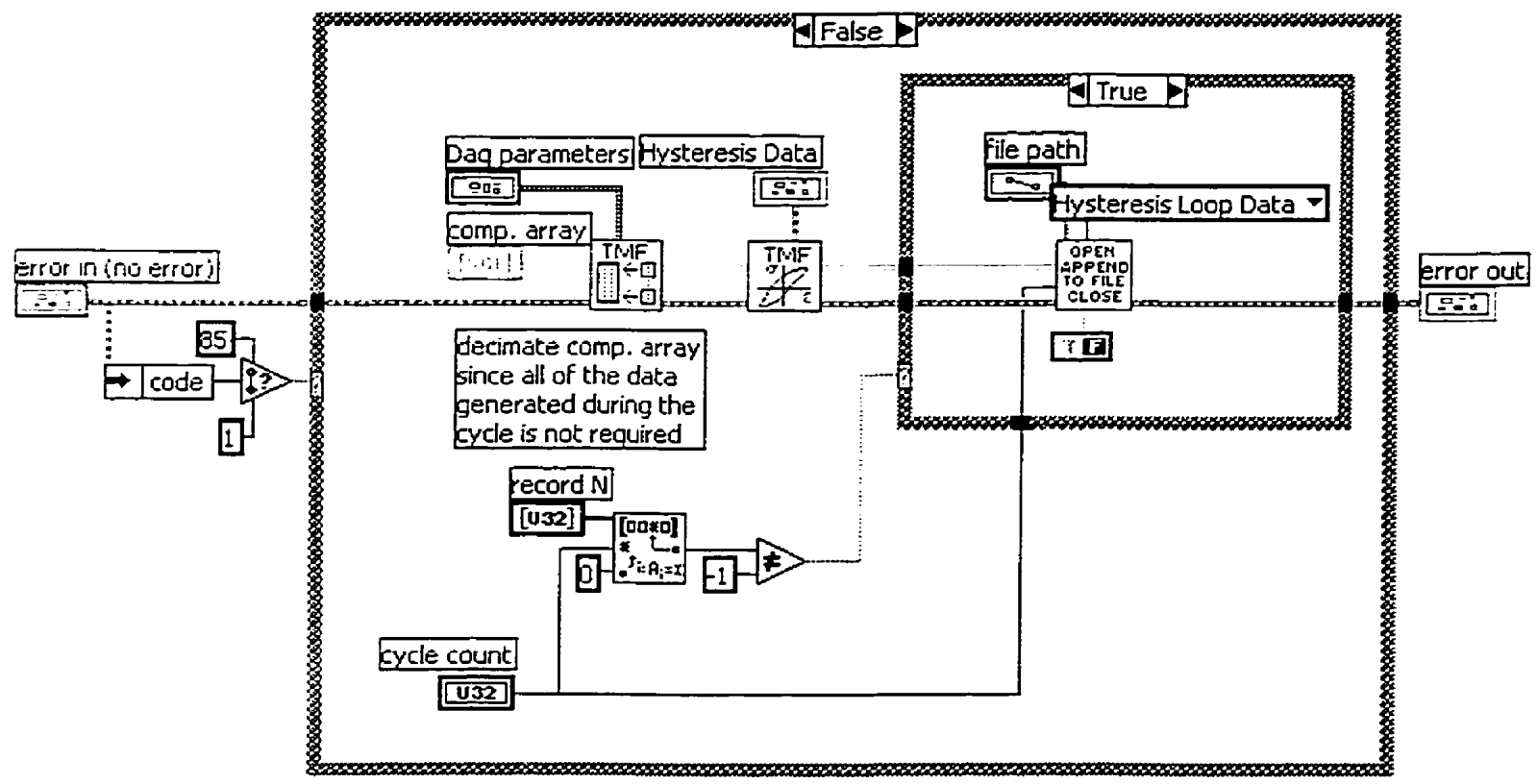

Figure A1. 34 - Block diagram for process hysteresis data.vi

\section{A 1.5.2.3.1. Decimate 2-D array.vi}

This VI is used to remove excess data (decimate) from the composite data array (array containing command and AI feedback data). Only data that corresponds to the data log interval specified by the user is retained for storage to the TMF hysteresis loop data log file. The first element of the command and AI scan arrays (time $=\sim 0$ seconds), and the array elements given by the loop index multiplied by the decimator minus I ([index * decimator] -1) are retained. The decimator is determined by the following equation:

array decimator $=$ update rate $*$ data $\log$ interval (updates) 
The VI loops from array index zero through to the number of array rows -1 to separate and retain the required array elements. This VI checks if an error has occurred with an error code in the range 1 to 85 . If an error code is in this range, the error cluster is passed through this VI without decimating the composite array. The connector pane and block diagram are illustrated in Figure A1. 35 and Figure A1. 36, respectively.

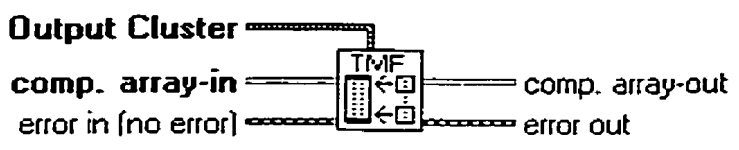

Figure Al. 35 - Connector pane for decimate 2-D array.vi.

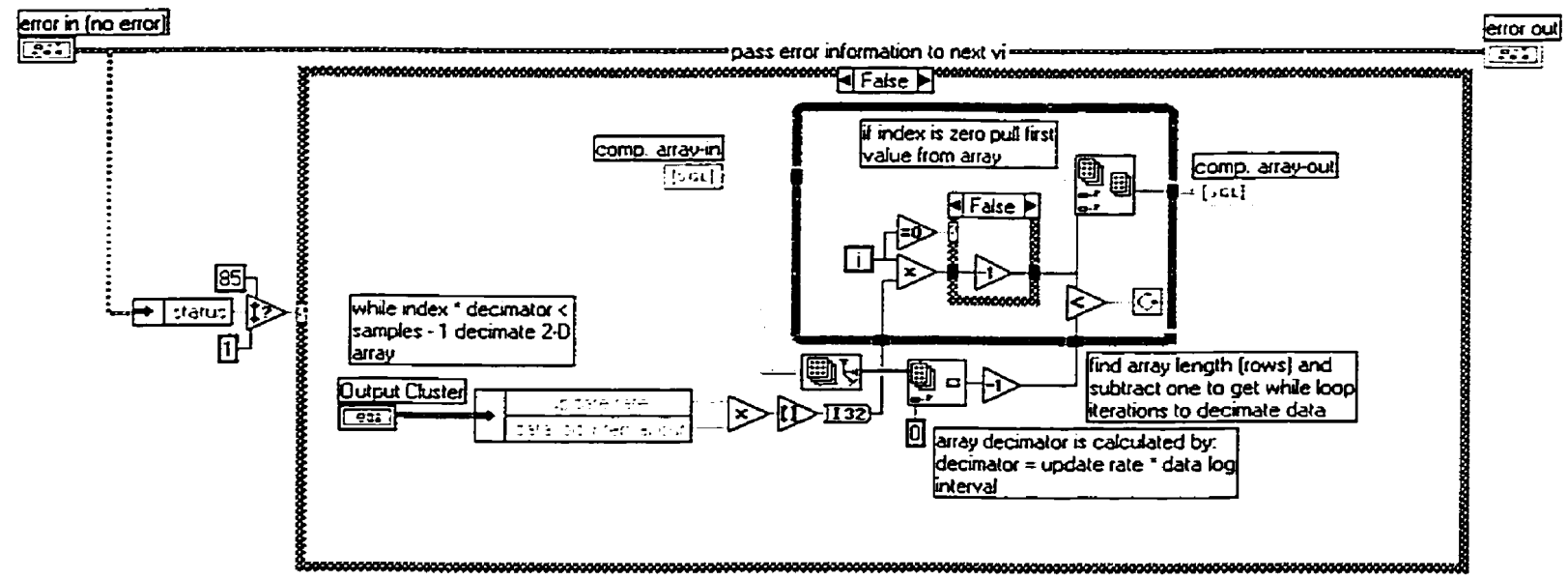

Figure Al. 36 - Block diagram for decimate 2-D array.vi.

\section{A1.5.2.3.2. Hysteresis.vi}

This VI updates the user display with the hysteresis loop for the most recently completed cycle. The hysteresis loop is plotted as load (V) versus mechanical strain (V) (notice that the thermal strain command has been subtracted to give the mechanical strain component). This VI checks for error codes in the range 1-85, if an error in this range is 
detected the hysteresis plot will not be updated and the error cluster is passed to the next VI. Note: if the user has changed the AI channel order, this VI must be updated with the correct channel information to display the correct information on the hysteresis plot. The connector pane and block diagram are illustrated in Figure Al. 37 and Figure Al. 38, respectively.

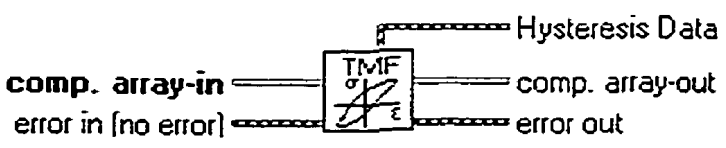

Figure Al. 37 - Connector pane for hysteresis.vi

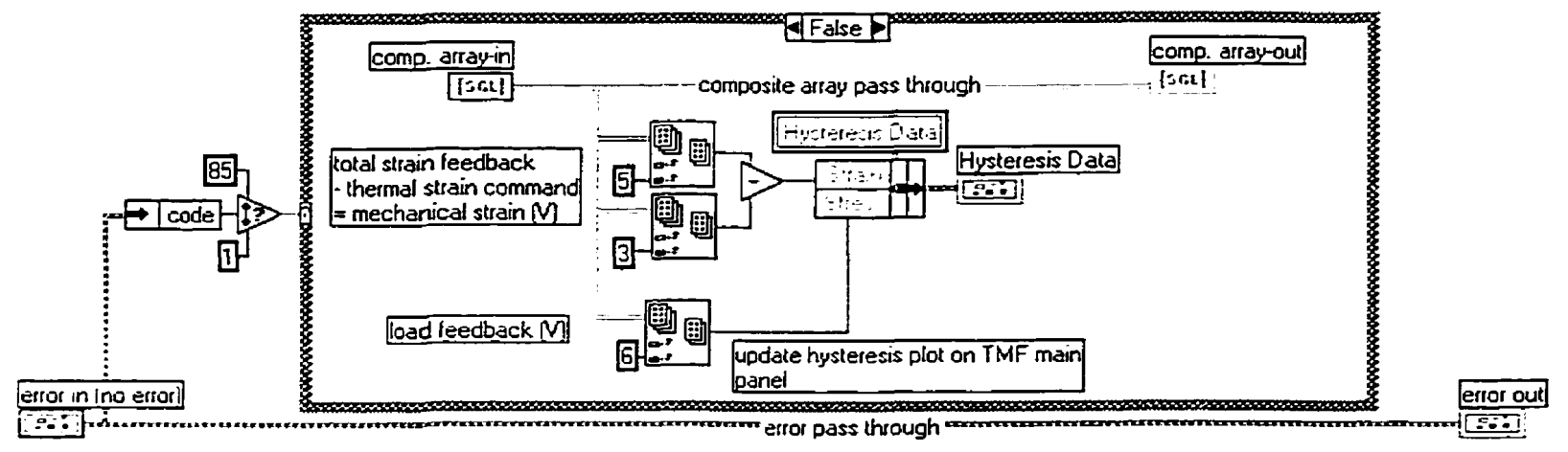

Figure Al. 38 - Block diagram for hysteresis.vi.

\section{A 1.5.2.4 Underpeak detector.vi}

This VI checks to see if the peak tensile load during the last cycle is greater than the under peak load specified by the user. If the peak load is less than the under peak load specified by the user, the 407 controller hydraulics are switched to low pressure, and the interlock chain will be opened (switching the hydraulics off). To control the hydraulics remotely, the 407 controller must be configured for remote operation. A call to 407 
change comm.vi is used to configure the 407 to accept a remote command. If a previous error is detected, this VI will pass the error onto the next VI without changing the hydraulic pressure setting. The connector pane and block diagram are illustrated in Figure Al. 39 and Figure A1. 40, respectively.

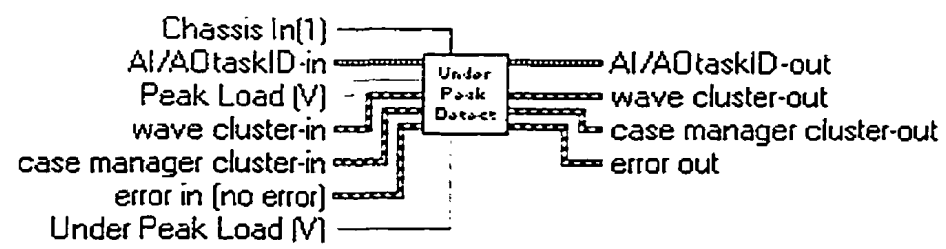

Figure Al. 39 - Connector pane for underpeak detector.vi.

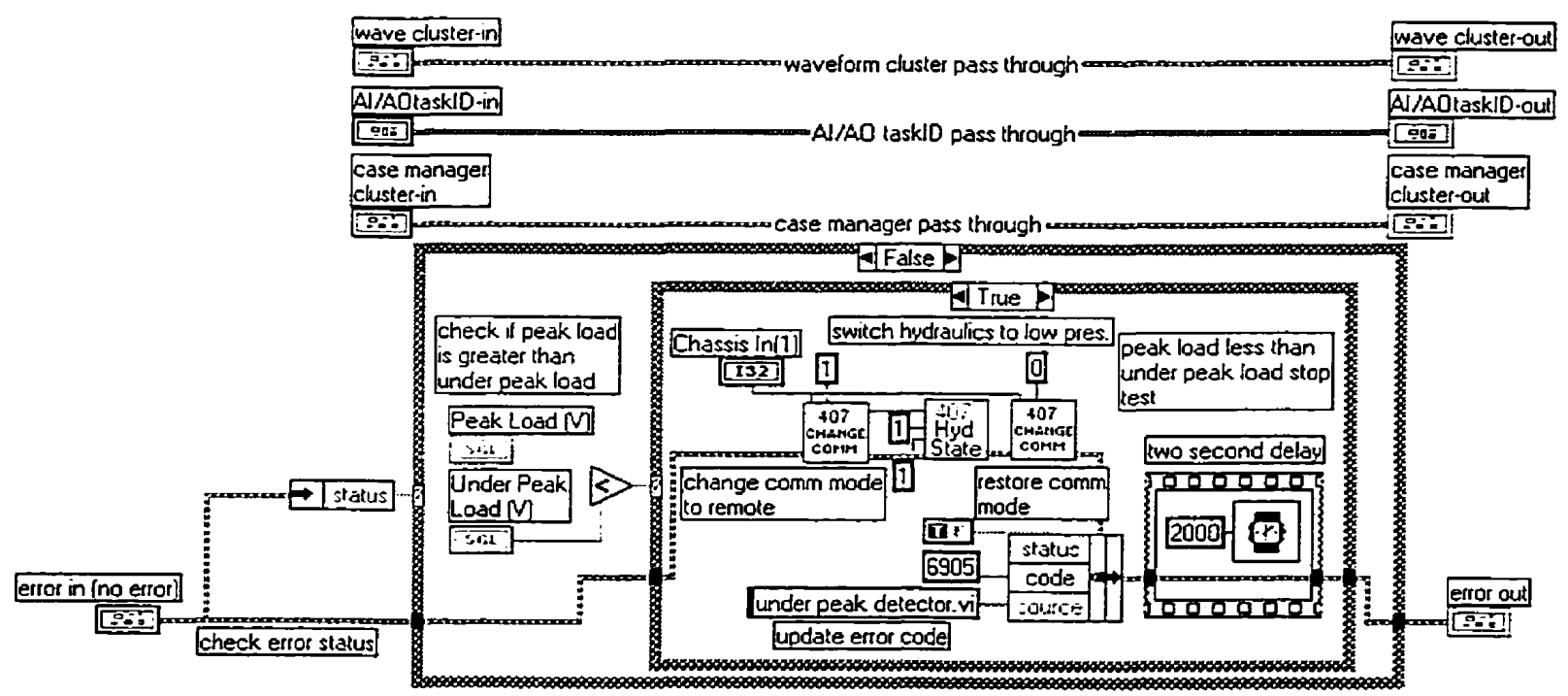

Figure A I. 40 - Block diagram for underpeak detector.vi.

\section{A1.5.2.4.1. 407 comm mode.vi}

This VI is used to switch the communications mode of the MTS 407 controller. Two communications options are available to the user, remote or local communications. In 
remote communications, the 407 controller keypad is disabled, and only commands sent via serial (RS-232) VO are accepted by the controller. In local communication mode, the keypad is enabled and serial L/O can only be used to query the controller for values (read only access). The connector pane and block diagram are illustrated in Figure A1.41 and Figure A1.42, respectively.

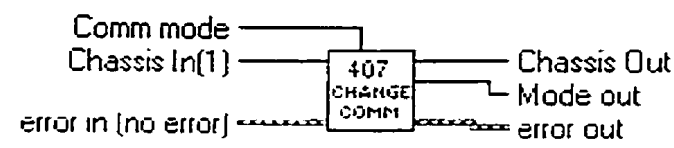

Figure A 1. 41 - Connector pane for 407 comm mode.vi.

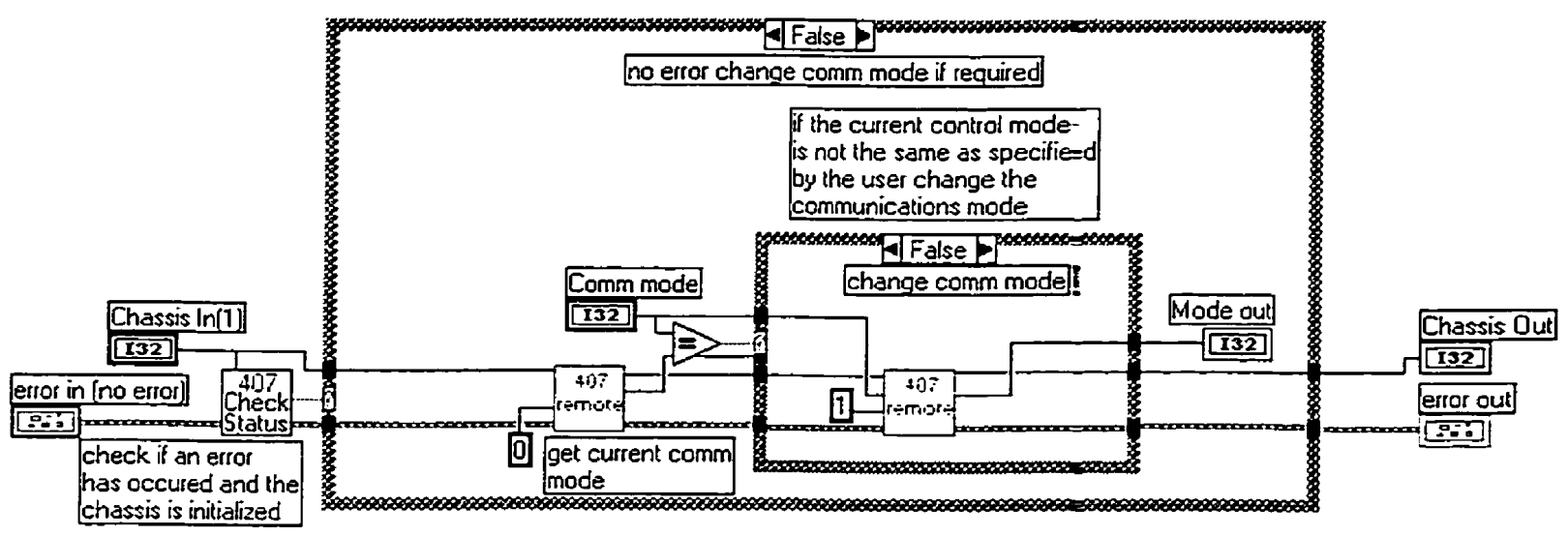

Figure A1. 42 - Block diagram for 407 comm mode.vi.

Note: When the control mode is changed to remote, a message will be displayed on the 407 controller DVM screen. This message does not signify an error, but rather warns the user that the communications mode has been switched to remo-te. 


\section{A 1.5.3 Control Loop VI's}

The TMF test application (program) control loop VI's are described in this section. The control loop VI's read the analog input data acquired during a scan of the analog input channels, averages the scan data, and updates the analog output channels with the temperature and total (or thermal) strain command signals once per loop iteration. Control loop execution control is facilitated by comparing the number of waveform samples -I to the loop index on each loop. If the index is greater than the waveform samples - 1 then the 'Continue' Boolean indicator value is set to false stopping the control loop. Control loop sub-VI's are also described in the following sections.

\section{A1.5.3.1 AI read and execution control.vi}

This VI calls AI Read VI to read eighty AI scans from the AI buffer for each channel in the channel list. If an error has occurred in a previous VI, the error information is passed onto the next VI without reading the scans contained in the AI buffer. The eighty scans contained in the 2-D scan array from AI Read are averaged on a per channel basis to give a final voltage reading. This VI also checks to see if the stop button on the user panel has been pressed, or the current while loop index greater than waveform samples - 1, or an 407 controller interlock is tripped. If an error in status is true, this VI passes the error information to the next VI. The connector pane and block diagram are illustrated in Figure A1. 43 and Figure A1. 44, respectively. 


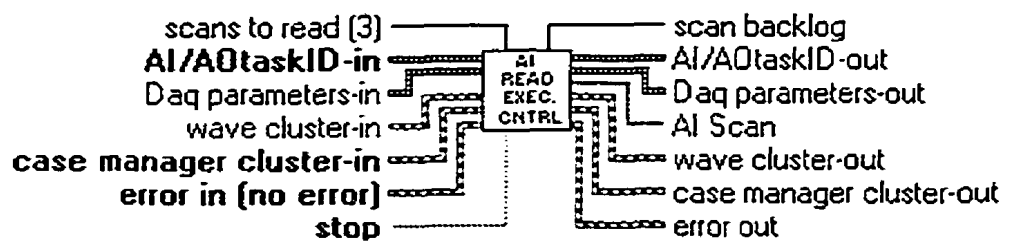

Figure A1. 43 - Connector pane for AI read and execution control.vi.

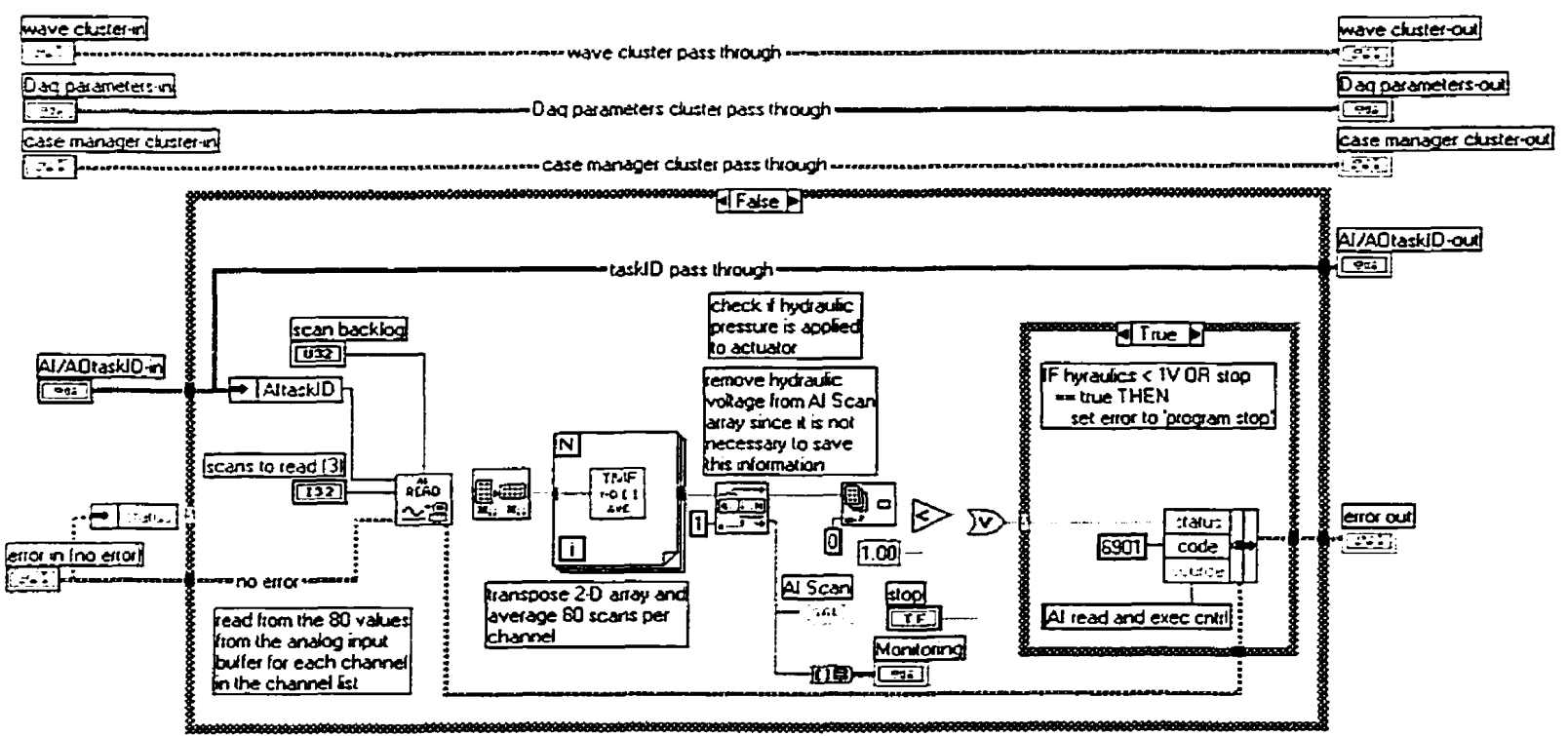

Figure A1. 44 - Block diagram for AI read and execution control.vi.

\section{A1.5.3.1.1. AI scan average.vi}

This VI calculates the average of a one-dimensional (1-D) array of single precision floating point values. The connector pane and block diagram are illustrated in Figure Al. 45 and Figure A1. 46, respectively.

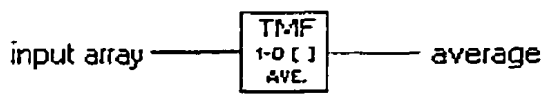

Figure A1. 45 - Connector pane for AI scan average.vi. 


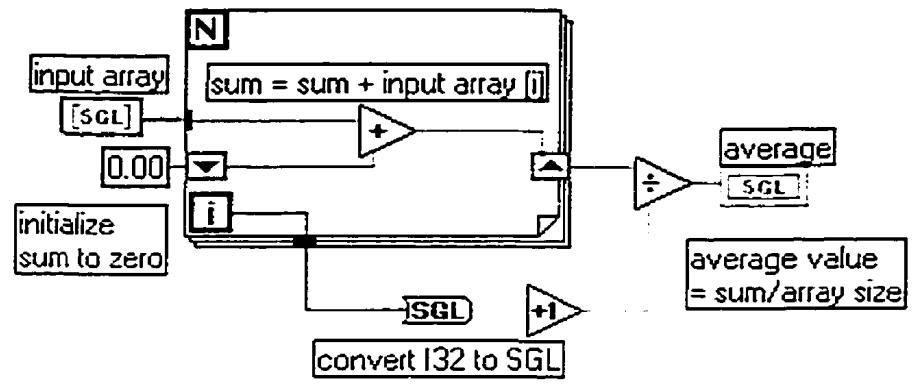

Figure Al. 46 - Block diagram for AI scan average.vi.

\section{A1.5.3.2 AO update manager.vi}

This VI controls the execution of the control loop of the TMF test software, updates the temperature and strain analog output channels, and updates the monitoring cluster on the TMF test software panel. Depending on the case, different command signal updates can be sent to the analog output channels.

Case U: This case holds the temperature constant at the mean test temperature (TMF test) or isothermal test temperature (IT-LCF test), and the strain command AO channel at zero volts for one cycle period.

Case 1: This case cycles the temperature (indexes temperature array by one on each loop) and outputs zero volts to the strain command channel.

Case 2: This case cycles the temperature and thermal strain (indexes temperature and thermal strain arrays by one on each loop).

Case 3: This case cycles the temperature and total strain (sum of mechanical and thermal strain) by indexing the temperature and mechanical strain arrays once per loop iteration. 
A composite array containing the command and AI scan signals is output from this VI for subsequent processing and storing to file. If an error is detected, this VI will not execute and pass the error information onto the next VI. The connector pane and block diagram are illustrated in Figure Al. 47 and Figure AI. 48, respectively.

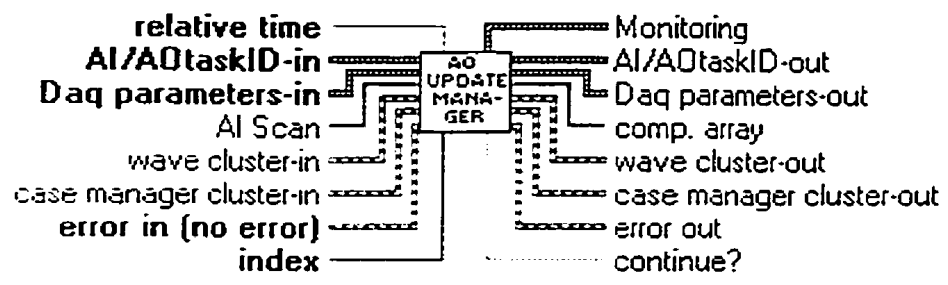

Figure A1. 47 - Connector pane for AO update manager.vi.

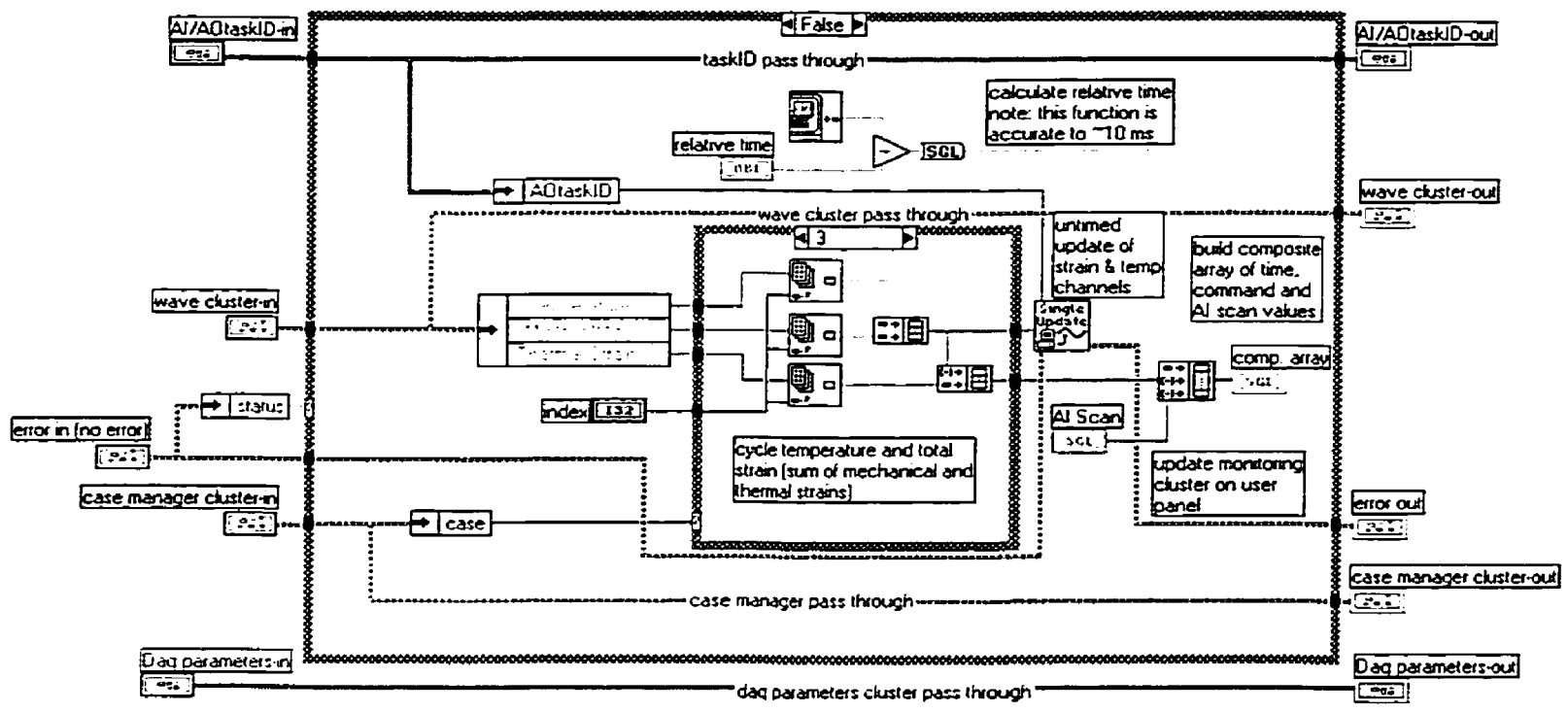

Figure Al. 48 - Block diagram for AO update manager.vi.

\section{A1.6 Program Termination VI's}

The program termination VI's are described in the following section. The simple error handler VI has not been included in the description of the program termination VI's, since it is a LabVIEW library function (see the LabVIEW User Manual). 


\section{A1.6.1 Stop test.vi}

This VI opens the interlock chain relays to stop a test in progress and clears analog input

DAQ resources. The connector pane and block diagram are illustrated in Figure A1. 49 and Figure A 1. 50, respectively.

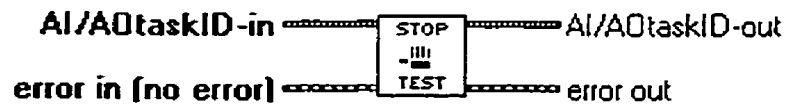

Figure Al. 49 - Connector pane for stop test.vi.

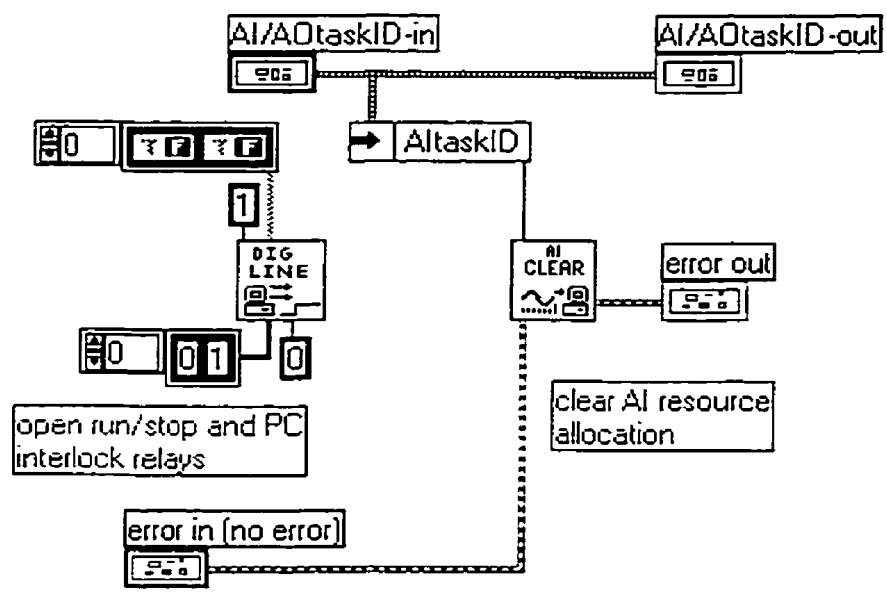

Figure Al. 50 - block diagram for stop test.vi.

Note: The write to digital line.vi does not have the error in cluster connected to the error in terminal of the sub-VI. If the error-in cluster is wired to digital-in.vi, the VI will not execute and open the interlock relays if an interlock event (program error, servohydraulic controller interlock) occurs. 


\section{Appendix 2}

\section{Procedures for Starting a TMF Test}

The following section outlines the procedure followed when setting up and conducting a TMF or IT-LCF test. Instructions are supplied for configuring the TMF test software and a step-by-step approach to conducting a test with the TMF testing facility is also presented. It is assumed that the reader is familiar with the mechanics of TMF testing, LabVIEW and the operation of the MTS 407 controller, Ircon Modline Plus infrared pyrometer, and the Lepel LSS 2.5 induction heater. The steps listed in this procedure are organized as they were followed in the present study.

\section{A2.1 Axial Extensometer Installation}

Two high temperature axial extensometers were used for testing. For IT-LCF tests a MTS $25 \mathrm{~mm}$ gage length (MTS model 632.56C-01) water-cooled, self-supporting extensometer was used. For TMF tests, a MTS $12 \mathrm{~mm}$ gage length (MTS model 632.41C-11) air-cooled extensometer was used. It is recommended that the minimum strain range capable of accommodating the thermal expansion and applied strain during a test is selected to maximize the feedback signal resolution and minimize interference from $\mathrm{RF}$ noise. For the work in this investigation smallest strain ranges for each extensometer were selected $(2.0 \%$ for $632.56 \mathrm{C}-01$, and $2.5 \%$ for $632.41 \mathrm{C}-11$ ). Before installing the axial extensometer, ensure the induction heater disconnect switch has been switched to the 'Off' position. The following steps must be taken into consideration. 
1. The limit of the fine zero adjustment of the MTS 407 controller is +- 2 VDC. The extensometer must be compressed slightly before heating to the test temperatures to ensure that the extensometer can be zeroed at the testing temperature. In the present investigation when heating IN738LC to $850^{\circ} \mathrm{C}$ for TMF tests, the $632.41 \mathrm{C}-11$ extensometer was compressed approximately 5.5 VDC at room temperature. For ITLCF tests at $950^{\circ} \mathrm{C}$ (with IN738LC), the $632.56 \mathrm{C}-01$ extensometer was compressed 6.5 VDC at room temperature. For other materials or temperature ranges, the amount of pre-compression required can be determined by zeroing the extensometer at room temperature and heating to the testing temperature. It is not recommended to adjust the coarse zero of the MTS 407 controller in order to zero the extensometer, as this can affect the extensometer calibration

2. Check the quartz rod vee-chisel tips carefully for damage before installing the extensometer. To prevent extensometer slip during the test, check that the quartz rods are seated correctly on the specimen. This can be verified by carefully pressing the top and bottom rods side-to-side, if an audible 'click' is heard, the quartz rods are not seated correctly.

3. Confirm that the cooling-air (or water) supply to the extensometer is operating. It is recommended that the extensometer be allowed to stabilize at the test temperature for half an hour before starting a test.

\section{A2.2 Specimen Pre-Oxidizing}

After the specimen and extensometer have been installed the specimen must be oxidized to obtain a stable oxide layer. This step ensures that the infrared pyrometer indicates the 
correct temperature, and a high friction surface is provided to prevent the axial extensometer from slipping during a test. The following steps should be followed:

1. Verify that the Lepel induction heater is configured for remote heat control. The three heat control switches on the induction heater panel should be set to 'Remote', 'Manual', and 'Aux' for remote 4-20 mA current input from the proportional temperature controller.

2. Check the induction heater interlock bypass switch is in the 'Bypass' position.

3. Switch the induction heater disconnect switch to the 'On' position and press the 'Reset' button on the generator panel to extinguish the cooling annunciating LED.

4. Because the major turns for the induction coil in the present study are located at the gage length extremes, the specimen must be slowly heated to the test temperature to prevent damage to the specimen and prevent the extensometer from slipping. Configure the Ircon proportional controller for manual output by switching the proportional controller switch to 'Manual'. Turn the 'Manual Adjust' potentiometer to approximately $40 \%$ output.

5. Press the 'Heat On' button on the induction heater control panel.

6. Slowly turn the Ircon proportional controller 'Manual Adjust' potentiometer to increase the induction heater power and bring the specimen up to the maximum test temperature.

7. When the specimen is at the maximum test temperature adjust the Ircon proportional controller 'Set Point' potentiometer while depressing the 'Set Point' switch to adjust the setpoint to the maximum test temperature. 
8. Switch the Ircon proportional controller mode to 'Automatic Internal' to maintain the specimen temperature at the setpoint value and hold at this temperature for the time required to pre-oxidize the specimen ( 2 hours pre-oxidizing time was used in this study).

\section{A2.3 Determining the Temperature Dependent Young's Modulus}

The following section outlines the procedure to determine the temperature dependent value of the Young's Modulus in tension. The purpose of measuring the temperature dependent value of Young's Modulus, is for computation of the inelastic stress-strain hysteresis loops for TMF tests. If an IT-LCF test is being performed, this step is not required. The temperature dependent Young's Modulus is not measured until the specimen pre-oxidizing step (see section A2.2) has been completed. Since the following procedure is conducted under force control one must be careful not to load beyond the yield point of the test specimen. To determine the temperature dependent Young's Modulus the following procedure is recommended:

1. Configure the MTS 407 controller function generator for a fully reversed triangular waveform (setpoint $=0 \mathrm{kN}$ ), and set the span value to a load value to prevent yielding the specimen at the test temperature. The triangular waveform frequency was set to $0.01 \mathrm{~Hz}$ in the present work.

2. Configure the MTS 407 controller limit values to ensure that the load can be applied without tripping an interlock. 
3. From LabVIEW, open the Acq to Spreadsheet File.vi, which is located in D:I. This LabVIEW program has been slightly modified from the Cont Acq to Spreadsheet File.vi that is located in the LabVIEW Examples Library. Refer to the VI in the LabVIEW Examples Library for information pertaining to this VI. This program was used in this study to acquire the Young's Modulus data. Configure the analog input channel string to channels 1-3. A view of the front panel for this VI is illustrated in Figure A2. 1 and shows the channel string configuration. This program will acquire data for the temperature, strain and load values.

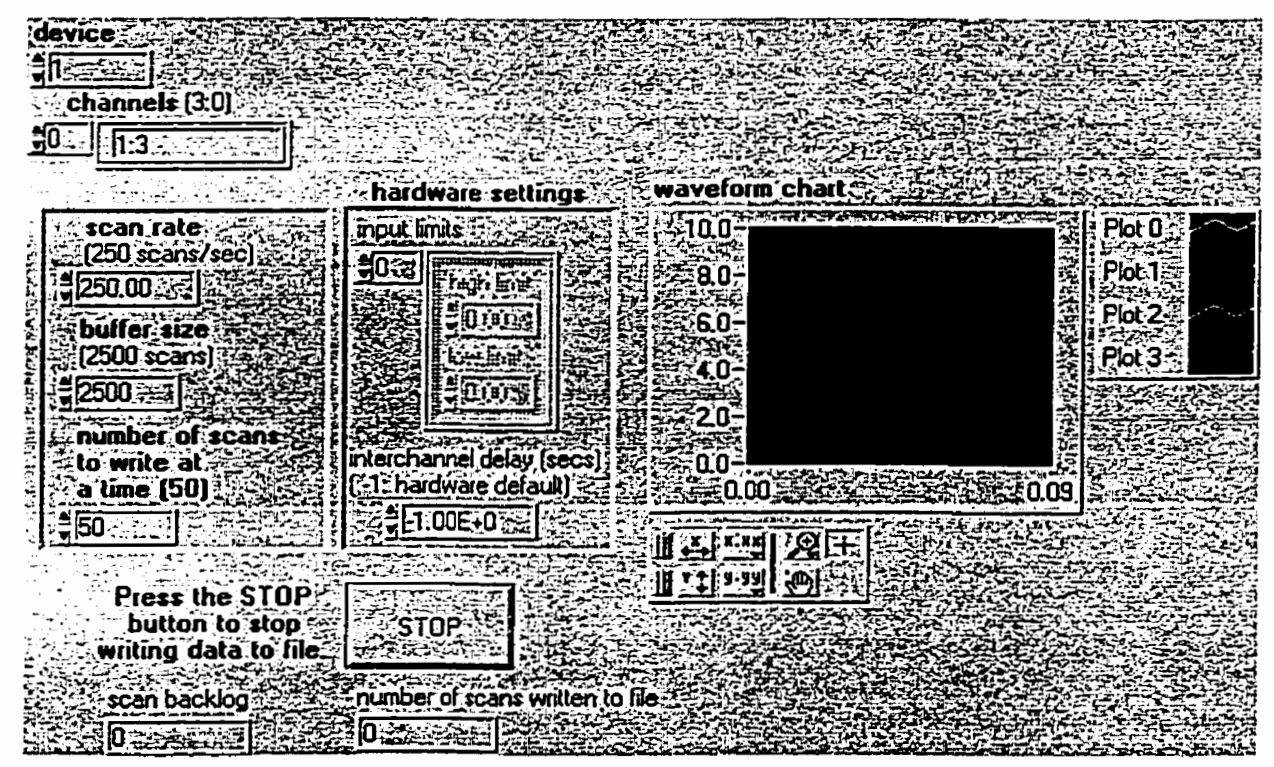

Figure A2. I - Front panel for Acq Spreadsheet File.vi. Notice the channels string in the top left corner of the panel has been configure for analog input channels 1-3.

4. Press the run button in LabVIEW to start Acq to Spreadsheet File.vi. The program prompts the user to input the path and filename for the spreadsheet file. After typing a valid path and filename, click 'OK' and press the 'Run' button on the MTS 407 controller. This will start the function generator and apply the triangular load 
waveform to the specimen. In the present study the triangular waveform was cycled three times before the 'Stop' button was pressed on the MTS 407 panel and the Acq to Spreadsheet File.vi front panel.

5. Change the Ircon proportional controller setpoint by rotating the 'Set Point' potentiometer while depressing the 'Set Point' button to a temperature less than the maximum test temperature and repeat steps 3 and 4. Note: the LabVIEW program does not have to be opened again as stated in step 3. During this study the Young's modulus was measured at temperature increments of $50^{\circ} \mathrm{C}$ between $750-950^{\circ} \mathrm{C}$ (i.e. $\left.950,900, \ldots 800,750^{\circ} \mathrm{C}\right)$.

\section{A2.4 Configuring the MTS 407 and Ircon Temperature Controllers}

After the specimen has been pre-oxidized and the temperature dependent Young's Modulus has been determined (if required), the 407 controller and infrared pyrometer must be configured for the test being conducted. The following outlines the procedure to be followed:

1. For a TMF test, set the Ircon proportional controller setpoint to the median test temperature by turning the 'Set Point' potentiometer while depressing the 'Set Point' switch until the median test temperature is displayed. Note: this step can be ignored for an isothermal LCF test provided that the proportional controller setpoint value is set to the correct temperature for the isothermal LCF test.

2. Adjust the fine zero for the extensometer, under the DC3 signal conditioning menu of the MTS 407 controller, to obtain zero volts at the current temperature (median test 
temperature for a TMF test, or the isothermal LCF test temperature for isothermal LCF tests).

3. Set the MTS 407 controller limits to suit the test. This includes the load, strain, displacement and error limits. The error limit should be set sufficiently low to stop a test if the extensometer slips; however, the error limit must be stringent enough to prevent tripping when switching from force to strain control. An error limit value set to $10 \%$ of the upper strain limit was found to be sufficient in this study to satisfy both these conditions.

4. Check that all interlocks for load, displacement, strain and error have been enabled.

5. Check that the grip hydraulic supply pressure has been adjusted to prevent backlash under the loads imposed during the test. The following equation can be used to determine the grip pressure for the MTS 647.10 wedge grips used in the present study:

$$
P(M P a)=\frac{12 * L(k N)}{A\left(\mathrm{~cm}^{2}\right)}
$$

where, $\mathrm{P}$ is the hydraulic supply pressure in $\mathrm{MPa}, \mathrm{L}$ is the maximum tensile or compressive load imposed during the test and $\mathrm{A}$ is the effective area of the hydraulic piston in the grips ( $66.45 \mathrm{~cm}^{2}$ for the MTS 647.10 grips). 


\section{A2.5 TMF Testing Software Configuration}

Before starting a TMF or isothermal LCF test, the TMF test software must be configured for the test being performed. The following section explains the software configuration procedure.

\section{A2.5.1 Configuring the TMF Testing Software Front Panel}

Several test settings must be configured on the front panel of the TMF testing software before pressing the 'Run' button in the LabVIEW environment. If one has not done so, open the TMF testing LabVIEW program. Figure A2. 2 illustrates the TMF testing software front panel the following parameters must be defined before pressing the 'Run' button:

1. Stab Cycles - Number of thermal stabilization cycles. Note: if an isothermal LCF test is being run, this control is ignored. The number of thermal stabilization cycles specified must be greater than one (1), but there is no limitation on the maximum number thermal stabilization cycles. In this investigation five (5) thermal stabilization cycles were used. By default the stab cycles control value is three (3) thermal stabilization cycles.

2. Record $\mathbf{N}$ - Array of user specified cycles to store in the TMF data log file. The record $\mathrm{N}$ array, contains the cycle numbers to be stored to the hysteresis data log file. The values specified in this array can be in any order and repeated values are ignored. As a minimum, logarithmic hysteresis loop recording intervals should be used as specified in ASTM E 606. The default value for this control is a null array, i.e. no cycles will be recorded to the hysteresis data log file. 
3. Under Peak Load (V) - The under peak tensile load, in volts. The test is stopped when the peak tensile load for a cycle is less than the under peak load value specified in this control. By default the value of this control is zero (0) volts, and a value can be specified at any point during the test if the under peak load value is not known in advance.

\section{A2.5.2 Front Panel Indicators}

The previous section explained the controls on the front panel that affect the TMF test configuration (see Figure A2.2). The front panel indicators are explained in this section:

- Hysteresis Plot - This is a plot of the hysteresis loop from the proceeding cycle, i.e. N-1. The plot is updated at the end of each cycle and represents a plot the load applied in volts versus the mechanical strain in volts.

- Monitoring - This cluster contains the 'real-time' value of the measured temperature (in degrees Celsius), load (in volts), and total strain (in volts). The values in this cluster are updated once per control loop iteration, i.e. during each scan of the analog input channels.

- $\mathbf{N}$ - Represents the current cycle count (N)

- Case - This is the program case that is currently executing in the TMF testing program.

- Backlog - This is the current analog input scan backlog in scans. This is useful for debugging purposes when an analog input error occurs. 
- Peak Load - This graph indicator illustrates the peak tensile load history during a test versus number of cycles.

\section{A2.5.3 Running the TMF Testing Program}

To run the TMF VI press the 'Run' arrow at the top of the screen (see Figure A2. 2). As the program runs, several interactive sub-VI front panels open prompting the user to input test parameters. The program can be stopped by pressing the stop button; however, if one of the interactive sub-VI panels is open the stop button will be ignored until the sub-VI panel is closed. The following sub-sections describe the interactive sub-VI panels that open in succession after the TMF testing program is started.

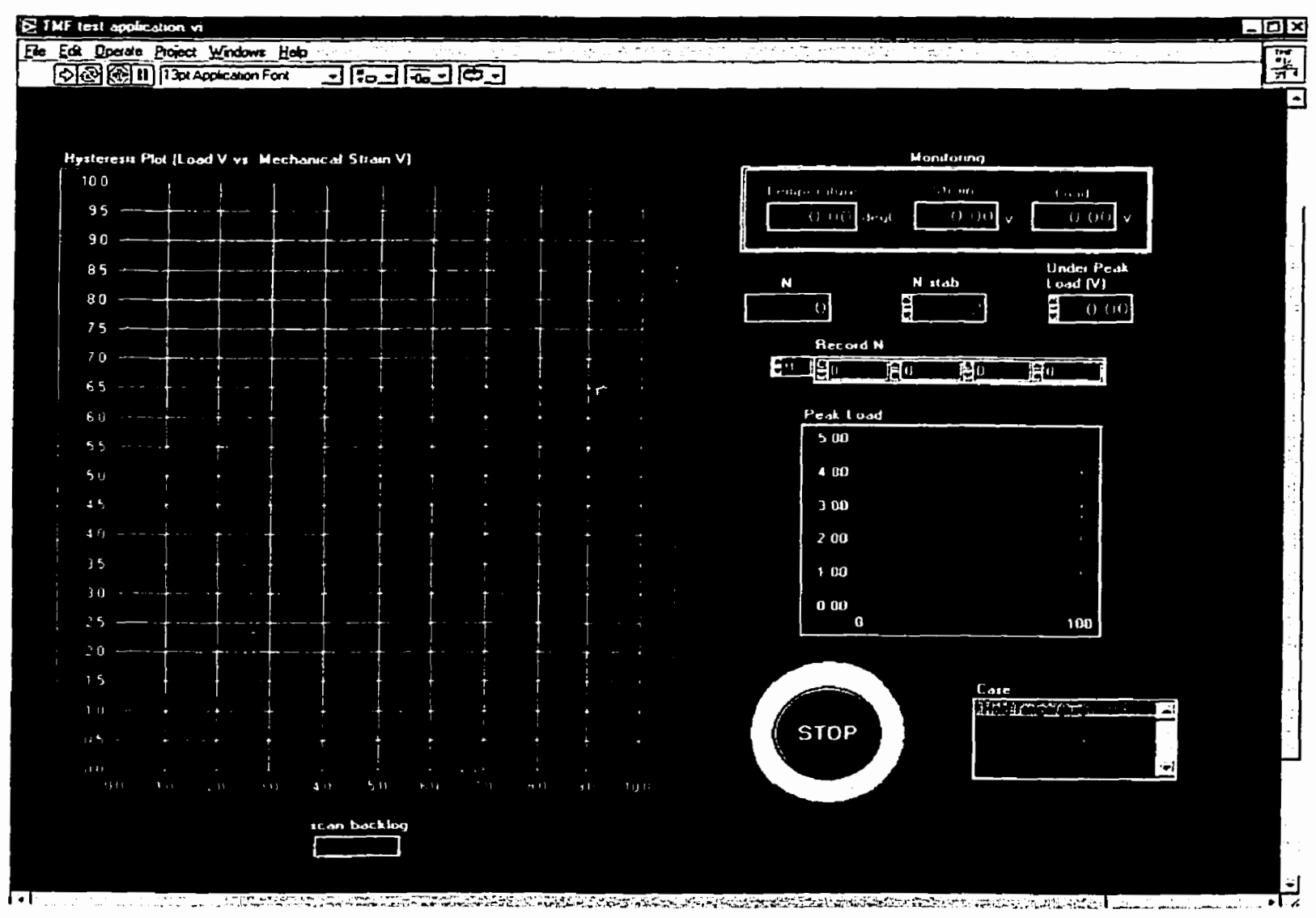

Figure A2. 2 - TMF testing application front panel. 


\section{A2.5.3.1 Initializing Serial Communications}

The first window to appear. after the TMF VI is started, allows the user to initialize serial communication between the host computer and the MTS 407 controller. This is done with the application $\mathbf{4 0 7}$ init port.vi which sets up the following parameters:

- baud rate (default 19200) and must match MTS 407 controller setting

- timeout duration, in ms (default 500)

- port number (default 1 :com 1 )

The front panel for 407 init port.vi is illustrated in Figure A2. 3. The default values for Port Number (Com L), Baud Rate (19200 bps) and Timeout (500 ms) are acceptable for the present 407 controller and host computer configuration.

To initialize the serial port with the default values, click the 'Init' button. The Initialized LED turns green to indicate the port initialization was successful. If the serial communication could not be established the Port Error LED turns red. If the Port Error LED turns red, the user should check to see if the correct communications (com) port was selected and that the baud rate matches the baud rate of the 407 controller (check 407 Communications menu for correct value). The Timeout value should not be changed from the default value (refer to MTS 407 LabVIEW Drivers/Applications Manual MTS part no. 151624-00-E). After the port is initialized correctly, press the 'Done' button to exit the sub-VI 


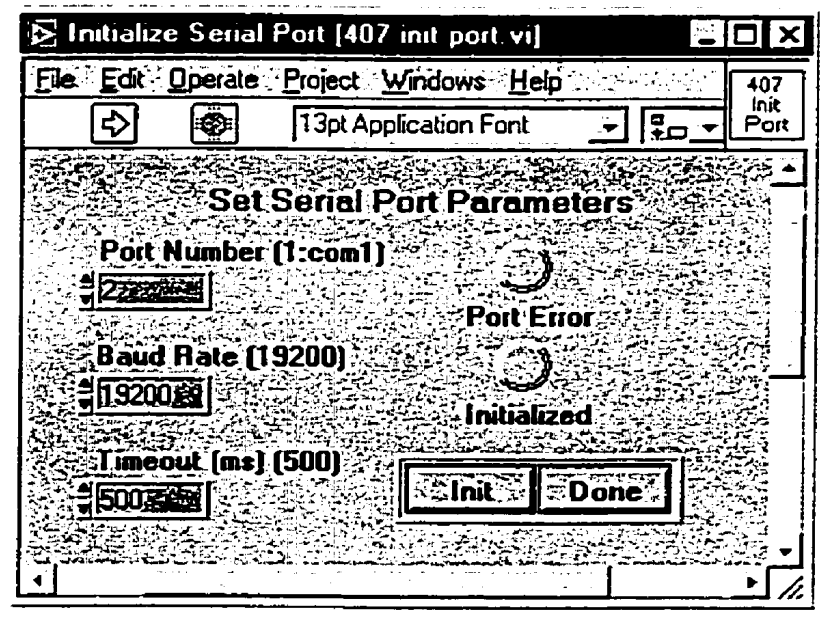

Figure A2. 3 - 407 init port.vi control panel.

\section{A2 5.3.2 Configuring Test Parameters}

After the serial port is successfully initialized, the TMF test configuration panel will open. The TMF test configuration front panel layout is illustrated in Figure A2. 4. It includes a selection area at the top to select the test parameters to adjust and an adjustment area for user to edit test settings. Objects below the selection area remain hidden until the user selects a test parameter to adjust from the 'TMF Parameter Menu' and clicks the 'Adjust' button. The controls shown in the selection area gray out after the 'Adjust' button is pressed.

Figure A2. 5 illustrates the adjustments window with all test parameters visible. When this VI is executed, only the item selected in the 'TMF Parameter Menu' will be visible after the 'Adjust' button is pressed. To configure other test parameters listed in the 'Test Parameter Menu' the click the 'Next' button to return to the selection area of the TMF config VI front panel. When all of the test parameters have been configured by the user click either 'Done' button in the adjustment area to close the front panel. 
Use the 'Test Parameter Menu', illustrated in Figure A2.4, to configure the following test parameters:

\section{TMF Test Config}

- Temperature strain phasing - type of temperature-strain relationship, choices are in-phase, out-of-phase, or isothermal.

- TMF cycle period - period of one strain and temperature cycle in seconds.

- Number of cycles - test duration in cycles.

\section{Data Acquisition}

- Data log interval - the interval between data points streamed to hard disk for storage.

- Frequency (updates/s) - analog output rate in samples per second (Note: Presently, the user can not adjust this control).

\section{Strain Config}

- Strain Span - the strain span (amplitude) in mm (Note: maximum value is found by querying 407 controller).

- Strain Setpoint - the strain setpoint (offset) in mm (Note: Presently, the user can not adjust this control)

\section{Temperature Config}

- Temperature Span - the temperature span (amplitude) in degrees Celsius (Note: if test is isothermal LCF the span control is ignored)

- Temperature Setpoint - the temperature setpoint (offset) in degrees Celsius (Note: if test is IT-LCF, this value must be set to the isothermal test temperature) 


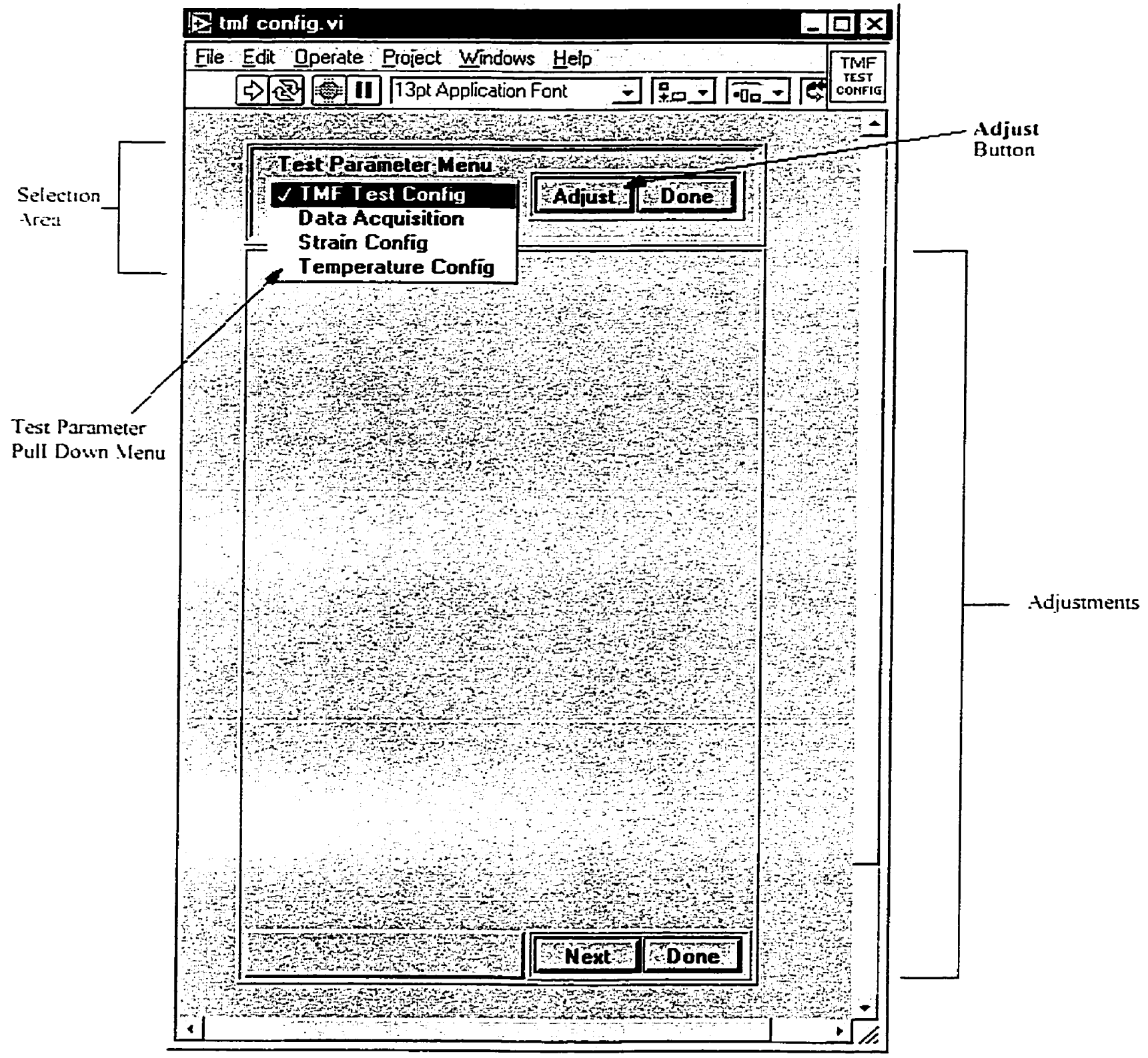

Figure A2. 4 - TMF config.vi front panel. 


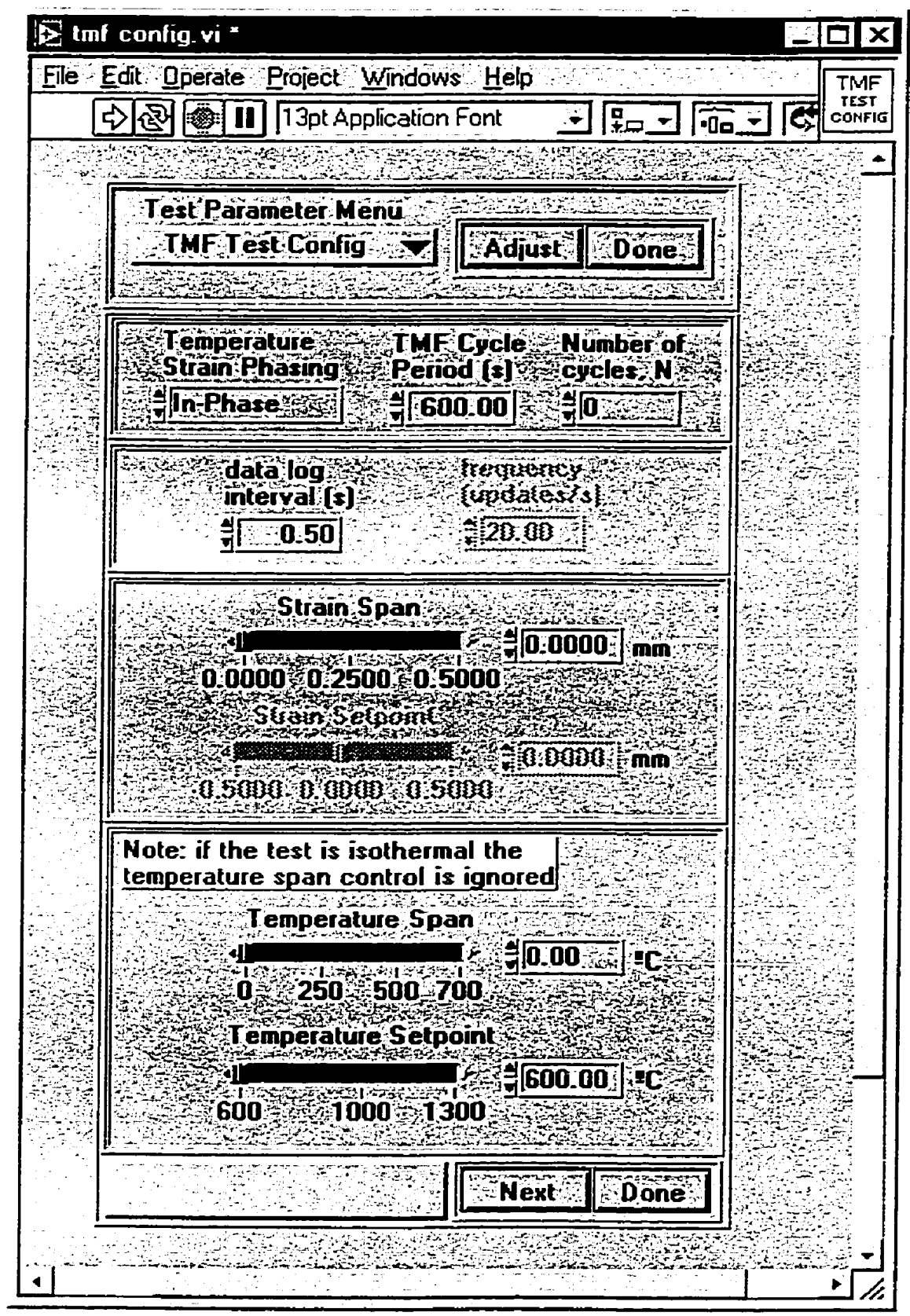

Figure A2. 5 - TMF config.vi front panel illustrating user configurable test parameters. 


\section{A2.5.3.3 Data File Se tup}

After the TMF test is configured by TMF config. vi, the TMF file configuration panel will open. The TMF file configuration panel is illustrated in Figure A2. 6. This VI creates and opens files for storing data acquired during a test. The user must specify a path and filename for each file path control shown on the front panel. Note that the specified path including drive letter should be specified in the path control, i.e. $<$ drive $>$ : $<$ directory $>k$ filename $>$, where directory must be an existing directory on the specified drive and filename must be a new filename to create (do not type $<>$ ). If a filename is not specified or one of the filenames is already in use, this VI will prompt the user to input a path and filename for any unspecified file path and name, or prompt the user to change the filename if it exists. The file controls specify the following:

- Pk/VI Load \& Strain Filename - the file and path for storing the peak/valley load and strain values, and tensile/compressive inelastic strain components.

- TMF Datalog Filename - the file and path for storing hysteresis loop data

- Thermal Strain Filename - the file and path for storing the thermal strain data used for thermal strain compensation

If an isothermal LCF test is being conducted, the 'Thermal Strain Filename' path control will be hidden on the front panel. 
The 'TMF Specimen \#' string control permits the user to input a specimen identification number that is appended to the data files. After the filenames are specified, click the 'Done' button to close the panel.

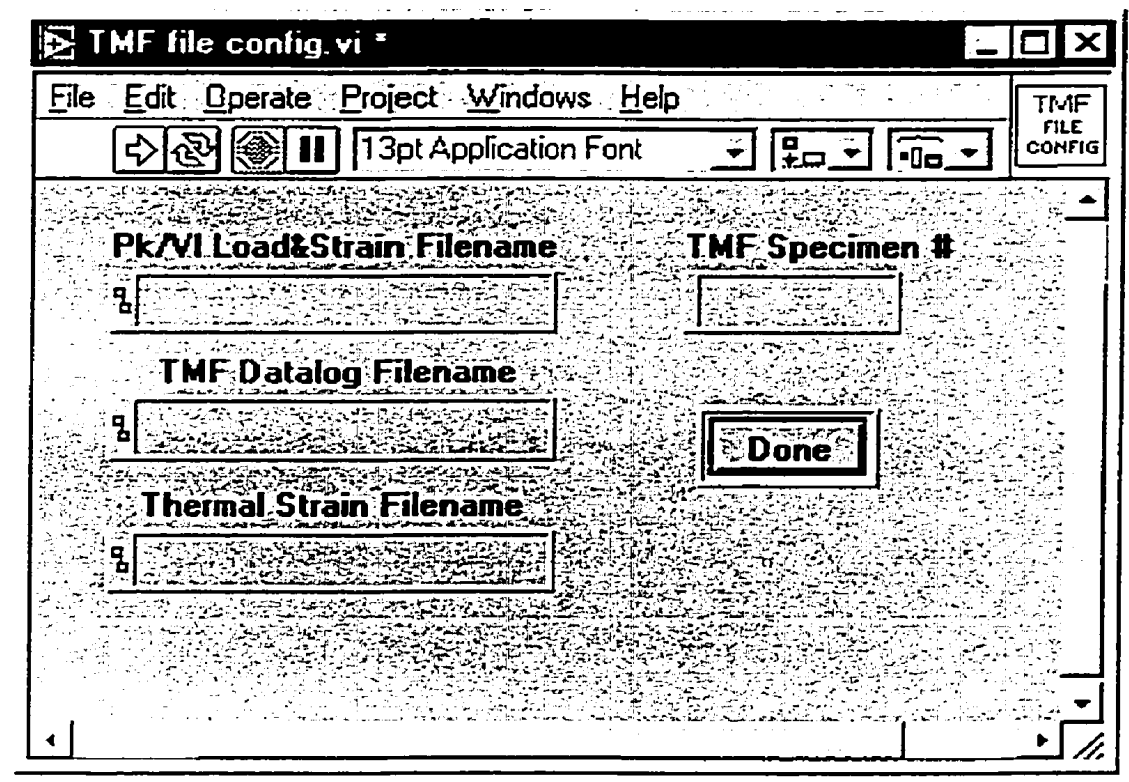

Figure A2. 6 - TMF file config.vi front panel.

\section{A2.6 Finalizing the Test Setup}

After the TMF test software is running several other actions are required by the user to finalize the test setup. For example, the user will be interactively prompted to switch the Ircon proportional temperature controller to accept an external setpoint signal from the data acquisition board, and later to switch the MTS 407 controller from force to strain control. The following section outlines the procedures to be followed when prompted to take an action during a test. 


\section{A2.6.1 Changing Setpoint Command Source and Zeroing Extensometer}

Before the hold case in the TMF test software in which the temperature is held constant for one period of the test cycle, the user will be prompted to switch the Ircon proportional controller command source to 'Automatic External' and to zero the axial extensometer. The prompt screen is illustrated in Figure A2. 7. When prompted for this task, the tollowing steps should be followed:

1. Switch the Ircon proportional controller command mode to 'Automatic External'. Wait approximately five (5) minutes before going to step 2 . The hold is included here to allow the temperature of the specimen to stabilize before zeroing the axial extensometer.

2. Adjust the fine zero for the extensometer, under the DC3 signal conditioning menu of the MTS 407 controller, to obtain zero volts at the current temperature (median test temperature for a TMF test, or isothermal LCF test temperature).

3. Switch the induction heater interlock bypass switch, located on the front panel of the Lepel induction heater, to 'Operate'. This enables the interlock connection between the MTS 407 controller and the induction heater.

4. Press the 'OK' button on the prompt window to continue, or press 'Cancel' to abort the test. 


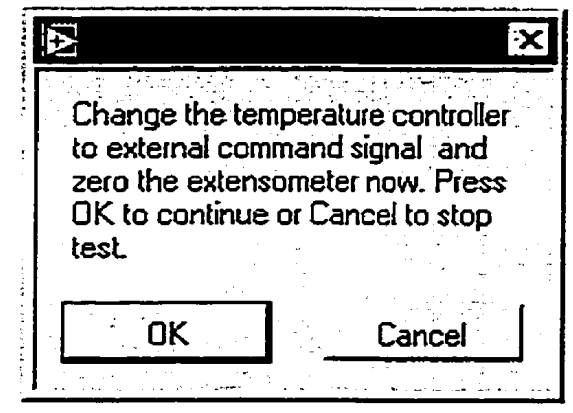

Figure A2. 7 - Window to prompting the user to switch the Ircon proportional controller to external command signal and to zero the axial extensometer.

\section{A2.6.2 Switching MTS 407 Controller to Axial Strain Control}

After the hold cycle, one of two things will occur depending on the type of test being conducted. If an isothermal LCF test is being conducted, immediately after the hold cycle the user will be prompted to switch the test controller to axial strain control. If a TMF test is being conducted, the TMF testing program will cycle the temperature for the number of thermal stabilization cycles specified by the user before prompting the user to switch to total axial strain control. The following subsections describe the procedure to be followed to switch the MTS 407 controller to axial strain control depending on the type of test being conducted.

\section{A2.6.2.1 Isothermal L CF Test}

Immediately after the hold cycle the user will be prompted to zero the axial extensometer and switch to axial strain control. This prompt window is shown in Figure A2. 8. The following steps should be followed: 
1. Adjust the fine zero for the extensometer, under the DC3 signal conditioning menu of the MTS 407 controller, to obtain a zero strain reading on the 407 controller DVM.

2. Press the hydraulic pressure 'Low' key on the MTS 407 control panel, wait one (1) second then press the hydraulic pressure 'Off' key. Note: this step is essential; if the hydraulics are not switched off when changing the feedback control mode unexpected actuator movement can occur.

3. Under the 'Controller' menu of the MTS 407 controller change the 'Fdback' menu item to the 'DC3' conditioner (extensometer signal conditioner).

4. Under the 'Configuration' menu of the MTS 407 controller, check the 'Cmd Src' menu item is configured to 'Ext In'. By default the MTS 407 controller is configured this way; however, if the menu item is configured to 'Composite' change it to 'Ext In'. This enables the MTS 407 controller to accept an external command source from the TMF testing software.

5. Check the error value on the MTS 407 controller DVM panel. If the error value is greater than $0.001 \mathrm{~mm}$, it is recommended that the test be aborted. If the error value is acceptable, press the hydraulic pressure 'Low' key followed by pressing the hydraulic pressure 'High' key on the MTS 407 control panel.

6. Switch the interlock bypass switch located on the MTS console to 'Off' to enable the external interlock chain.

7. Press the ' $\mathbf{O K}$ ' button on the prompt window to continue, or press 'Cancel' to abort the test.

8. Configure the MTS 407 controller DVM2 to display the peak/valley load history for the test. 


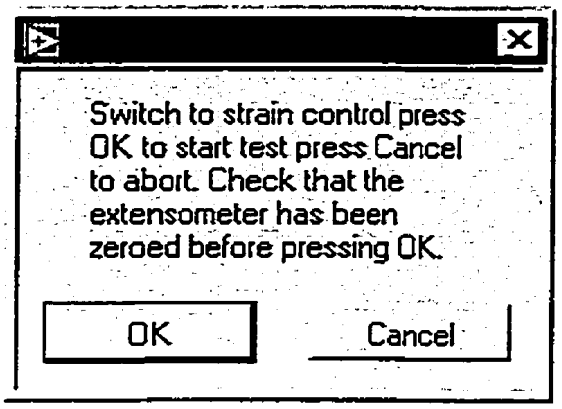

Figure A2. 8 - Window prompting the user to zero the axial extensometer and switch the MTS 407 controller from force to strain control.

\section{A2.6.2.2 TMF Test}

Immediately after the temperature has been cycled the user specified number of thermal stabilization cycles, the user will be prompted to switch the MTS 407 controller to total axial strain control. This prompt window is illustrated in Figure A2. 9. The following section summarizes the procedure to be followed:

1. Press the hydraulic pressure 'Low' key on the MTS 407 control panel, wait one (1) second then press the hydraulic pressure 'Off' key. Note: this step is essential; if the hydraulics are not switched off when changing the feedback control mode unexpected actuator movement can occur.

2. Under the 'Controller' menu of the MTS 407 controller change the 'Fdback' menu item to the 'DC3' conditioner (extensometer signal conditioner).

3. Under the 'Configuration' menu of the MTS 407 controller, check the 'Cmd Src' menu item is configured to 'Ext In'. By default the MTS 407 controller is configured this way; however if the menu item is configured to 'Composite' change it to 'Ext 
In'. This enables the MTS 407 controller to accept an external command source from the TMF testing software.

4. Check the error value on the MTS 407 controller DVM panel. If the error value is greater than $0.001 \mathrm{~mm}$, it is recommended that the test be aborted. If the error value is acceptable, press the hydraulic pressure 'Low' key followed by pressing the hydraulic pressure 'High' key on the MTS 407 control panel.

5. Switch the interlock bypass switch located on the MTS console to 'Off' to enable the external interlock chain.

6. Press the ' $\mathbf{O K}$ ' button on the prompt window to continue, or press 'Cancel' to abort the test.

7. Configure the MTS 407 controller DVM2 to display the peak/valley load history for the test.

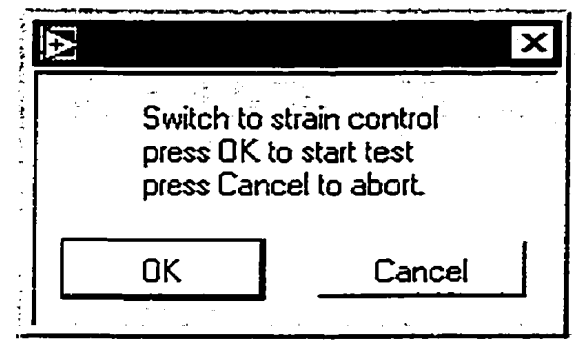

Figure A2. 9 - Window prompting the user to switch the MTS 407 controller to axial strain control.

\section{A2.7 Actions to be Taken During a Test}

During a TMF or isothermal LCF test the user is required to perform several tasks. For example, the load limits should be adjusted periodically to account for the cyclic 
softening or hardening behavior of the test material. The following subsections outline the actions that should be taken by the user during a test.

\section{A2.7.1 Load Limit Adjustment}

The load limits must be adjusted periodically during an isothermal LCF or TMF test to account for the cyclic softening or hardening of the test material. Both the peak and valley loads displayed on the MTS 407 controller DVM and the peak load history displayed on the TMF test software front panel should be consulted as a reference point for configuring the load limits. Special consideration should be given to the upper load limit, as it should be stringent engough to prevent a 'runaway' condition if a fatigue crack occurs outside of the extensometer gage section, and high enough to compensate for cyclic hardening effects.

\section{A2.7.2 Under-Peak Load Adjustment}

Periodically during a test the under-peak load control, located on the TMF test software front panel, requires adjustment during a test to account for the cyclic softening or hardening behavior of the test material. The peak load history graph on the TMF test software (see Figure A2.2) should be consulted to estimate an under-peak value that will stop the test based on the load drop used as the failure criteria.

\section{A2.8 Actions to be Taken After a Test}

After a TMF or isothermal LCF test stops the user must take several actions in-order to re-enable the test apparatus for another test. These actions are listed as follows: 
1. Switch the induction heater interlock bypass switch, located on the front panel of the Lepel induction heater, to the 'Bypass' position. Switch the induction heater disconnect switch to the 'Off' position.

2. Switch the external interlock bypass switch, located on the MTS console, to the 'On' position.

3. Clear the 'E-Stop', and 'load' interlocks, and disable the 'stain' interlocks in the MTS 407 'Interlock' menu.

4. Remove the axial extensometer.

5. Remove the test specimen from the grips. 


\section{Appendix 3}

\section{Visual Basic Post-Test Processing Software and Forms}

The following program was written in Visual Basic V 6.0 for the purpose of post-test processing of the hysteresis loop and peak/valley load and strain data files to engineering units. Both the source code and forms have been provided in this appendix. The output files generated by this program is formatted to import directly into Tecplot as an ASCII *.dat file.

\section{A3.1 Source Code}

\section{A3.1.1 frmTMFProcess}

'Must declare all variables

Option Explicit

- Private variables for the input and output file stream refnums

Private InFilenum. OutFilenum As Integer

Private Sub Form_Load()

'Add items to cboProcFileType combo list box on program loading cboProcFileType.AddItem "Hysteresis", 0 cboProcFileType.AddItem "Peak Stress", 1

'Make cmdProcess button unuseable crndProcess. Enabled $=$ False

'hide progress bar visible

pbrProgress. Visible $=$ False

End Sub

Private Sub cboProcFileType_Click()

Dim InFilePath As String

'If the selected file to process is hysteresis or peak stress then prompt

'the user to open a file by calling frmOpenFile and get the filename selected 
' in frmOpenFile otherwise exit the subroutine

If cboProcFileType.Text $=$ " Then

Exit Sub

Else

- Disable the cboProcFileType combo list box until done processing cboProcFileType. Enabled = False

- Open the frmOpenFile form to prompt the user for the input file frmOpenFile.Caption = "Open TMF data file for processing" frmOpenFile.Show I

InFilePath $=$ frmOpenFile.Tag

' If the input file path is not a path then prompt the user to enter a file otherwise open the file and enable the cmdProcess button

It InFilePath $=$ "'" Then

- Enable cboProcFileType for user selection

cboProcFileType. Enabled = True

'Warn the user to enter a valid file path

MsgBox "You must pick a file to open to continue!", vbExclamation, "Error"

Exit Sub

Else

'Enable the cmdProcess button so that the software can begin processing

the tile

cmdProcess. Enabled $=$ True

'Get a free file number

InFilenum $=$ FreeFile

'Open input file

Open InFilePath For Input As InFilenum

On Error GoTo errorl 'next command can cause an error goto errorl on error pbrProgress. Max $=$ FileLen (InFilePath $)+1$ 'set maximum progress bar value End If

End If

Exit Sub exit subroutine

errorl:

Close InFilenum ' close input file

Exit Sub 'exit subroutine

End Sub

Private Sub cmdProcess_Click()

Dim OutFilePath As String

'Open the frmOpenFile form to prompt the user for the output file frmOpenFile.Caption = "Save TMF File As"

frmOpenFile.Show I

OutFilePath $=$ frmOpenFile.Tag 
- If the output file path is not a path then prompt the user to enter a file otherwise open the file and disable the process button If OutFilePath $="$ " Then

'Warn the user to enter a valid file path

MsgBox "You must pick a file to open to continue!", vbExclamation, "Error"

Exit Sub

Else

- Disable the cmaProcess button so that the software can begin processing the tile and enable cboProcFileType for another selection by the user emdProcess. Enabled $=$ False

cboProcFileType. Enabled $=$ True

' Get a free file number

OutFilenum $=$ FreeFile

'Open output file

Open OutFilePath For Output As OutFilenum

- It the file type being processed is a hysteresis data file call 'process_HystData otherwise call process_PeakStress

If cboProcFileType. Text = "Hysteresis" Then Else Call process_HystData

End If

Call process_PeakStress

End If

End Sub

Prialc Sub process_HystData()

Const $\mathrm{NCol}=6$

Dim n(6) As Single

Dim cycleCnt, count, Nvar As Integer

Dim specimenID, cycle, a, strg 1 , Numb(6), asciicode As String

Dim IWP, CWP, LI As Long

Dim cp, c As Integer

- Initialize count and number of output variables to 0 and 3 respectively count $=0$

Nvar $=3$

Initialize cycle to null string

cycle $=" "$

pbrProgress. Visible $=$ True ' make progress bar visible

frmTMFProcess. Refresh 'refresh the screen

'Read the first line of text from the input file and extract the specimen ID

' contained in the first 6 characters of the file

Line Inpul \#InFilenum, specimenID

specimenID = Left (specimenID. 6)

'Call PrintTitle to print the title of the data to be processed to the 
'output file

Call PrintTitle(specimenID)

"If the specimenID starts with the two letters IT then the test was isothermal

'prompt the user to enter the Young's modulus of the material by showing the

'Young's modulus dialog (dIgYoungMod) and set the form tag (frmTMFProcess.tag)

'to reflect the test type frmTMFProcess.tag $=1$ if isothermal and 0 otherwise

If UCase(specimenID) Like "IT*" Then

frmTMFProcess. Tag $=1$

Else

frmTMFProcess. Tag $=0$

End If

dlg YoungMod.Show I

' If the user did not enter the Young's modulus correctly an error value 0 ' is 'retumed in the Young's modulus dialog tag and the program exits this subroutine If digYoungMod.Tag $=0$ Then GoTo error I

'show calculation constants dialog box

dlgCalcConst.Show 1

'if calculation constants dialog tag is zero then user pressed cancel button If digCalcConst. Tag $=0$ Then GoTo errorl

- Read the second line of the input file and store the value at the right of the 'ampresand in the cycle count 'cycle' string. Note the original subroutine for

'reading a tab delimited text file character by character was written by

'Tak Terada, i.e. the lines of code listed below. This subroutine has been

'modified for use on TMF hysteresis data files by Scott Yandt.

read line by line until no null string or EOF detected

Do Until cycle $>$ "' Or EOF(1)

Line Input \#InFilenum. cycle

Loop

- Find the length of the string read in

$\mathrm{LI}=$ Len(cycle)

' Cycle is all of the characters to the right of the ampresand

cycle $=$ Right $($ cycle,$(\mathrm{LI}-\mathrm{I}))$

'Call PrintZone sub to print the zone header with the cycle number

Call PrintZone(cycle)

'Get the current position of the output file pointer initial write position (IWP)

IWP $=$ Seek(OutFilenum)

Call PrintlJK to print the number of points written to the file

Call PrintlJK(count)

Call PrintDT to print the varible number type string to file

Call PrintDT(Nvar)

' Read data from the input file until EOF = true

Do Until EOF(1)

'Reset the character pointer to zero

$\mathrm{cp}=0$

'Read the next line from the input file

Line Input \#InFilenum, a

-If the first character of the line is an ampresand then the current line 
' contains the cycle count else if the character is NOT a null string or

'newline then read the string character by character and separate into

'variables

If $\operatorname{Left}(a, 1)=$ "\&" Then

'The cycle count is stored in the characters following the ampresand

$\mathrm{LI}=\operatorname{Len}(\mathbf{a})$

cycle $=$ Right(a. $(\mathrm{Ll}-1))$

- Move to the position pointed to by the initial write position (IWP) file

- pointer

Seek OutFilenum, [WP

'Call PrintIJK subroutine to print the number of points processed to the

'output file at the postion pointed to by the initial write position (IWP)

'output file pointer

Call PrintIJK(count)

'Return to the last file position pointed to by the current write position

'(CWP) output file pointer

Seek OutFilenum, CWP

- Call PrintZone subroutine to print the next zone number to file

Call PrincZone(cycle)

'Set the initial write position (IWP) file pointer to the current position

'in the output file

IWP $=$ Seek(OutFilenum)

'Call PrintIJK to print the number of points processed to the output file

Call PrintJJK(count)

'Call PrintDT to print the variable number types to the output file

Call PrintDT(Nvar)

Reset the points processed counter to 0

count $=0$

Elself Left(a, 1) $\diamond " "$ And Left(a. 1) $\diamond \operatorname{Chr}(13)$ Then

' Read the input string a character by character and convert to

'numbers and store in a variables array

For $\mathrm{c}=0$ To NCol

'reinitialize Numb array and strg I variables to null string

$\operatorname{Numb}(\mathrm{c})=" \cdot$

$\operatorname{strgl}=" "$

Do

increment character pointer

$\mathrm{cp}=\mathrm{cp}+\mathrm{I}$

$\operatorname{strg} l=\operatorname{Mid}(a, c p, l)$

'If the next character is null string exit do loop

If $\operatorname{strg} l=" '$ Then Exit Do

'if the next character is a tab exit do loop

asciicode $=$ Asc(strg 1 )

If asciicode $=9$ Then Exit Do

$\mathrm{Numb}(\mathrm{c})=\mathrm{Numb}(\mathrm{c})+\mathrm{strg} 1$

Loop

'The c'th element of the variables array is the value of the converted string

$n(c)=\operatorname{Val}(\operatorname{Numb}(c))$

Next c

'Increment points written to the output file counter

count $=$ count +1

'Call HystDataReduction subroutine to process the variables array

Call HystDataReduction(n()) 
- Call PrintHystDataToFile to print the processed variables array to

'the output file

Call PrintHystDataToFile(n())

- Set the current write position (CWP) file pointer to the current

'position in the output file

CWP $=$ Seek(OutFilenum)

pbrProgress. Value $=$ Seek (InFilenum) ' update progress bar

End If

Loop 'loop until EOF is true

Move to the position pointed to by the initial write position file pointer,

'print the number of points processed to file and return to the current write

'position in the output file

Seek OutFilenum, IWP

Call PrintUJK(count)

Seek OutFilenum, CWP

'Close the input and output files

Close InFilenum

Close OutFilenum

pbrProgress. $V$ isible $=$ False hide progress bar

Exit Sub "exil subroutine

errorl:

'close the input and output files

Close InFilenum

Close OutFilenum

pbrProgress. Visible $=$ False ${ }^{\prime}$ hide progress bar

Exit Sub 'exit subroutine

End Sub

Private Sub HystDataReduction(m() As Single)

Dim ET2, ET, EC, Tstrain, Mstrain, Stress, InEstrain, Estrain As Single

'Set the coefficients for the Young's modulus equation as a function of temperature

ET2 = Val(dIgYoungMod.txtT2)

$\mathrm{ET}=\mathrm{Val}(\mathrm{dlg}$ YoungMod.txtT)

$E C=\operatorname{Val}($ dlgYoungMod.txtC)

- Mechanical strain is the total strain feedback $[\mathrm{m}(5)]$ - the thermal strain command

- $[\mathrm{m}(3)]^{*}$ the maximum strain reading for the selected range of the extensometer $/ 10$

Mstrain $=((m(5)-m(3)) *$ Val (dlgCalcConst.txtStrainRange $)) / 10$

'Total strain is the total strain feedback $[\mathrm{m}(5)] *$ the maximum strain reading

- for the selected range of the extensometer $/ 10$

Tstrain $=(\mathrm{m}(5) *$ Val (dlgCalcConst.txtStrainRange $)) / 10$

'Stress is the force feedback $[\mathrm{m}(6)] *$ maximum load for the selected load cell

'range $/ 10$

Stress $=(\mathrm{m}(6) * \operatorname{Val}(\mathrm{dlgCalcConst.txtLoadRange)}) /(10 * \operatorname{Val}($ dlgCalcConst.txtArea $))$

- Elastic strain is the stress / the Young 's modulus

Estrain $=$ Stress $/((E T 2 * m(4) \wedge 2+E T * m(4)+E C) * 1000)$

- Inelastic strain is the mechanical strain - the elastic strain

InEstrain = Mstrain - Estrain

- Format the output values to \%.5f format

$\mathrm{m}(0)=$ FormatNumber(Mstrain, 5)

$\mathrm{m}(\mathrm{l})=$ FormatNumber(InEstrain, 5) 
$m(2)=$ FormatNumber (Tstrain, 5)

$\mathrm{m}(3)=$ FormarNumber(Stress, 5)

End Sub

Private Sub PrintHystDataToFile(n) As Single)

'Print the hysteresis loop data to the output file

Print \#OutFilenum, $n(0)$; $n(1) ; n(2) ; n(3)$

End Sub

Private Sub Print Title(ID)

Dim Title. Var As String

'If the user specifies to process hysteresis loop data then var is the string

'shown below else it is the other string

If cboProcFile Type.Text $=$ "Hysteresis" Then

Var = "Variables = " + Chr(34) + "Mstrain" + Chr(34) + Chr(34) + "InEstrain" + Chr(34) + Chr(34) +

"Tstrain" + Chr(34) + Chr(34) + "stress" + Chr(34)

Else

Var $=$ "Variables $="+\operatorname{Chr}(34)+$ "Cycle" + Chr(34) + Chr(34) + "maxTStrain" + Chr(34) + Chr(34) +

"minTStrain" + Chr(34) + Chr(34) + "maxMStrain" + Chr(34) + Chr(34) + "minMStrain" + Chr(34) +

Chr(34) + "maxStress" + Chr(34) + Chr(34) + "minStress" + Chr(34) + Chr(34) + "StressRange" + Chr(34)

+ Chr(34) + "MStrainRange"

End If

'Set the title to the specimen ID

Title $=$ "Title $=$ " + Chr(34) + CStr(ID) + Chr(34)

'Print the title and variable list to the output file

Print \#OutFilenum, Title

Print \#OutFilenum, Var

End Sub

Private Sub PrintZone(Cnt)

Dim zone As String

'Create the zone string

zone $=$ "Zone $\mathrm{T}=$ " $+\mathrm{Chr}(34)+\mathrm{Cnt}+\mathrm{Chr}(34)$

'Print the zone string to the output file

Print \#OutFilen um, zone

End Sub

Private Sub PrintDT(num)

Dim DT, Var As String

Dim i As Integer

'Initalize var to a null string

Var $=" "$

-For $\mathrm{i}=0$ to the number of variables create the variable type list

For $\mathrm{i}=0$ To nurn 
Var $=$ Var + " Double"

Next $\mathrm{i}$

$\mathrm{DT}=$ " DT $=("+\mathrm{Var}+") "$

-Print the variable list to the output file

Print \#OutFilenum. DT

End Sub

Private Sub PrintIJK(points)

Dim IJK As String

'Create the IJK string

$\mathrm{IJK}=" \mathrm{I}=$ " + CStr(points) + ", $\mathrm{J}=\mathrm{I}$, " + "K $=\mathrm{L} "$

'Print the IJK string to the output file

Print \#OutFilenum, IJK

End Sub

Private Sub process_PeakStress()

Const $\mathrm{NCol}=6$

Dim n(8) As Single

Dim count. Nvar As Integer

Dim specimenID, a, strgl, Numb(10), asciicode, zone As String

Dim IWP. CWP. LI As Long

Dim ep.c As lnteger

show calculation constants dialog box

dlgCalcConst.Show I

'if calculation constants dialog tag is zero then user pressed cancel button If dlgCalcConst. Tag $=0$ Then GoTo errorl

pbrProgress. Visible $=$ True ' make progress bar visible

'Itialize the points counter (count) $=0$, number of variables (Nvar) $=8$

"and zone = "PkVIData"

count $=0$

Nvar $=8$

zone = "PkVIData"

' Read a line in from the input (peak valley data) file

Line Input \#InFilenum, specimenID

'SpecimenID $=$ the left 6 characters of the first line read in from the file

specimenID $=$ Left (specimenID, 6)

'Print the title and zone names to the output file

Call PrintTitle(specimenID)

Call PrintZone(zone)

- Set the initial write postion (IWP) to the current value of the output file

pointer

IWP $=$ Seek(OutFilenum)

- Print the IJK and variables list (DT) to the outpuc file

Call PrintlJK(count)

Call PrintDT(Nvar)

' Read data from the input file until EOF

Do Until EOF(1) 
' Initalize the character postion pointer to zero

$\mathrm{cp}=0$

'Read a line in from the input data file

Line Input \#InFilenum, a

'For character 0 to number of columns read character by character and

'build the variables array

For $\mathrm{c}=0$ To NCol

- Initialize the character array and strg I variables to null string

Numb(c) $=" \cdot$

$\operatorname{strg} l={ }^{* *}$

'Read input string character by character until null string or tab

Do

Increment the character pointer

$\mathrm{cp}=\mathrm{cp}+1$

$\operatorname{strg} \mathrm{l}=\operatorname{Mid}(\mathrm{a}, \mathrm{cp} . \mathrm{l})$

'If the character is null string or tab then exit the do loop

If strgl $=$ "" Then Exit Do

ascilcode $=$ Asc(strgl)

If asciicode $=9$ Then Exit Do

$\mathrm{Numb}(\mathrm{c})=\mathrm{Numb}(\mathrm{c})+\operatorname{strg} \mathrm{I}$

Loop

'Build variable array

$\mathrm{n}(\mathrm{c})=\mathrm{Val}(\mathrm{Numb}(\mathrm{c}))$

Next c

'Increment points written counter

count $=$ count +1

'Convert data to engineering units and write to file

Call PkVIDataReduction(n())

Call PrintPkVIDataToFile(n())

pbrProgress. Value $=$ Seek $($ InFilenum $){ }^{\circ}$ update progress bar

Loop 'loop until EOF is true

'Goto the intial write position in the output file and write the IJK data to the

'output file

Seek OutFilenum, IWP

Call PrintIJK(count)

Close the input and output files

Close InFilenum

Close OutFilenum

pbrProgress. Visible $=$ False ${ }^{\circ}$ hide progress bar

Exit Sub 'exit subroutine

errorl:

'Close the input and output files

Close InFilenum

Close OutFilenum

pbrProgress. Visible $=$ False ' hide progress bar

Exit Sub 'exit subroutine

End Sub

Private Sub PkVIDataReduction(m() As Single)

Dim i As Integer 
- Process the data

$m(0)=m(0)$

'Calculate the peak total tensile strain

$\mathrm{m}(\mathrm{l})=(\mathrm{m}(\mathrm{l}) * \mathrm{Val}(\mathrm{dlgCaI}$ Const.txtStrainRange $)) / 10$

'Calculate the peak total compressive strain

$\mathrm{m}(2)=(\mathrm{m}(2) * \mathrm{Val}(\mathrm{dlgCalcConst.txtStrainRange)}) / 10$

'Calculate the peak mechanical tensile strain

$\mathrm{m}(3)=(\mathrm{m}(3) * \mathrm{Val}(\mathrm{dlgCalcConst.txtStrainRange})) / 10$

'Calculate the peak mechanical compressive strain

$\mathrm{m}(4)=(\mathrm{m}(4) * \mathrm{Val}(\mathrm{dlgCalcConst.txtStrainRange})) / 10$

- Calculate the peak tensile stress

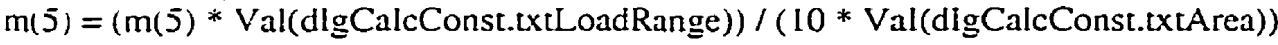

'Calculate the peak compressive stress

$\mathrm{m}(6)=(\mathrm{m}(6) * \mathrm{Val}(\mathrm{dlgCalcConst.txtLoadRange)}) /(10 * \mathrm{Val}(\mathrm{dlgCalcConst.txtArea}))$

Calculate the stress range

$m(7)=m(5)-m(6)$

'Calculate the mechanical strain range

$m(8)=m(3)-m(4)$

'Format the output values to \%.5f format

For $\mathrm{i}=0$ To UBound(m())

$m(i)=$ FormatNumber $(m(i), 5)$

Nexti

End Sub

Private Sub PrintPkVIDataToFile(m() As Single)

Dim out As String

Dim i As Integer

'Intialize the out string to null string

out $="$ "

'For $i=0$ to array length of $m$ build output string

For $i=0$ To $\operatorname{UBound}(m(), 1)$

out $=$ out $+" "+\operatorname{CStr}(\mathrm{m}(\mathrm{i}))$

Next i

'Print processed data to output file

Print \#OutFilenum, out

End Sub

Private Sub mnuExit_Click()

' If the exit item in the file menu is accessed then unload the dlgYoungMod and

'frmOpenFile forms, end the program and exit

Unload dlgYoungMod

Unload dlgCalcConst

Unload frmOpenFile

Unload frmAbout

End

End Sub

Private Sub mnuAbout_Click() 
frmAbout.Show

End Sub

\title{
A3.1.2 frmOpenFile
}

\author{
-All variables MUST be declared \\ Option Explicit \\ Private Sub Form_Load() \\ -Fill the cbofileType combo box \\ cboFileType.AddItem "All files (**)" \\ cboFileType.AddItem "Text files (*.TXT)" \\ cboFileType.AddItem "Data files (*.DAT)" \\ - Initialize the cboFileType combo box to item \#0 (All files) \\ cboFileType.ListIndex $=0$ \\ - Update the lblDirName label with the path \\ IblDirName. Caption $=$ dirDirectory. Path
}

End Sub

Private Sub drvDrive_Change()

' Set an error trap

On Error GoTo DriveError

' Change the path of the directory list box to the new drive dirDirectory. Path $=$ drvDrive. Drive

Exit Sub

DriveError:

'An error occured, inform the user and restore the original drive MsgBox "Drive error!", vbExclamation, "Error"

drvDrive.Drive $=$ dirDirectory.Path

Exit Sub

End Sub

Private Sub dirDirectory_Change()

'A new directory was selected by the user so update the path of the file ' list box

filFiles.Path $=$ dirDirectory.Path

'Update the lblDirName label

IbiDirName. Caption $=$ dirDirectory.Path

End Sub 
Private Sub cboFileType_Click()

'Change the pattern of the file list box according to the file type 'selected by the user

Select Case cboFileType.ListIndex

Case 0

tilFiles.Pattern $=* * * *$

Case 1

tilFiles.Pattern $=$ "*.TXT"

Case 2

filFiles.Pattern $=" *$.DAT"

End Select

End Sub

Private Sub filFiles_Click()

- Update the txtFileName text box with the file name that was just selected txtFileName.Text $=$ filFiles. FileName

End Sub

Private Sub filFiles_DbIClick()

'If the user double clicks the filename update txtFileName text box

' with the filename and execute the $\mathrm{cmdOK}$ procedure

txtFileName. $T$ ext $=$ filFiles. FileName

cmdOK_Click

End Sub

Private Sub cmdOK_Click()

Dim Path, PathAndName As String

'If no file is selected, tell the user to pick a file and exit procedure

If txtFileName. Text $=$ " "Then

MsgBox "You must first select a file!"

Exit Sub

End If

'Make sure the Path ends with backslash 1

If Right(filFiles.Path, 1) $\diamond$ "I" Then

Path = filFiles.Path $+" \mathrm{"}$

Else

Path = fillFiles.Path

End If

'Extract the path and name of the selected file

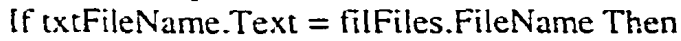

PathAndivame $=$ Path + filFiles.FileName

Else

PathAndName $=$ Path + txtFileName.Text

End If

'Set the form tag to the path and name of the file and hide the form 
frmOpenFile.Tag $=$ PathAndName

frmOpenFile.Hide

End Sub

Private Sub cmdCancel_Click()

'The user has selected cancel set the form tag to null string and hide the form frmOpenFile.Tag $=" "$

frmOpenFile.Hide

End Sub

\section{A3.1.3 dlgCalcConst}

-All varibles must be declared

Option Explicit

Private Sub CancelButton_Click()

dlgCalcConst. $T a g=0$ set calculation constant tag to 0 to indicate cancellation

dlgCalcConst.Hide 'hide dialog box

End Sub

Private Sub cboAreaUnits_LosLFocus()

Dim Response As String

Dim units As Integer

units $=$ cboAreaUnits.ListIndex 'set units to current combo box list index

If units $=1$ Then

'promt user to convert units

Response = MsgBox("Do you wish to convert the units to $\mathrm{mm}^{\wedge} 2$ ?", vbYesNo, "Convert Area Units")

If Response $=$ vbYes Then

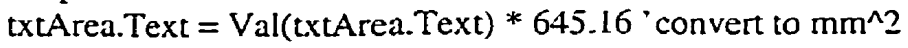

cboAreaUnits.ListIndex $=0$ ' change combo box to $\mathrm{mm}^{\wedge} 2$

End If

End If

End Sub

Private Sub cboLoadUnits_LostFocus()

Dim Response As String

Dim units As Integer

units $=$ cboLoadUnits.ListIndex 'set units to current combo box list index

If units $=1$ Then

'promt user to convert units

Response $=$ MsgBox ("Do you wish to convert the units to N?", vbYesNo, "Convert Load Units")

If Response $=$ vbYes Then

txtLoadRange.Text $=$ Val (txtLoadRange.Text $) * 4.4482216$ ' convert to Newtons

cboLoadUnits. ListIndex $=0$ ' change combo box to Newtons

End If 
End Sub

Private Sub Form_Activate()

'reset calculation constant dialog tag to $\mathrm{I}$ when form is active window dlgCalcConst. Tag $=1$

End Sub

Private Sub Form_Load()

add menu items to load, strain and area units combo boxes

cboLoadUnits.AddItem " $N$ ", 0

cboLoadUnits.AddItem "lbs", 1

cboStrainUnits.AddItem " $\mathrm{mm} / \mathrm{mm}$ ", 0

cboStrain Units.AddItem "in/in", I

cboAreaUnits.AddItem " $\mathrm{mm}^{\wedge} 2$ ", 0

cboAreaUnits.AddItem "in^2", 1

'initialize Load/Strain Range and Area to default values

cxtLoadRange. Text $=$ "50000.00"

txtStrain Range. Text $=$ "0.02"

txt.Area. Text $=" 31.669 "$

set combo boxes for units to default units

cboLoadUnits. ListIndex $=0$

cboStrain Units. Listlndex $=0$

cboAreaUnits.List Index $=0$

'set calculation constant dialog tag to 1 when form loads

dlgCalcConst. Tag $=1$

End Sub

Private Sub txtArea_Change()

Dim check As Boolean 'boolean value for insnumeric function

Dim lastval As Single 'last valid value entered by user

'check to see if current load range is a valid number

check $=$ IsNumeric(txtArea.Text)

If check $=$ False Then

lastval = Val(txtArea. Text) $\quad$ value is not valid store last valid number

GoTo errorl 'goto error handling code

Else: Exit Sub valid number exit subroutine

End If

errorl:

MsgBox "You must enter a valid numeric value", vbExclamation 'error message

txtArea. Text $=$ lastval 'set displayed load range to last valid number

\section{End Sub}

Private Sub oxtLoadRange_Change()

Dim check As Boolean 'boolean value for insnumeric function

Dim lastval As Single "last valid value entered by user 
'check to see if current load range is a valid number

check = IsNumeric (txtLoadRange. Text)

If check $=$ False Then

lastval = Val(txtLoadRange. Text $)$ 'value is not valid store last valid number

GoTo errorl

' goto error handling code

Else: Exit Sub

- valid number exit subroutine

End If

errorl:

MsgBox "You must enter a valid numeric value", vbExclamation 'error message

txtLoadRange. $T$ ext $=$ lastval ' set displayed load range to last valid number

End Sub

Private Sub txtStrainRange_Change()

Dim check As Boolean 'boolean value for insnumeric function

Dim lastval As Single 'last valid value entered by user

'check to see if current strain range is a valid number

check $=$ IsNumeric(txtStrainRange. Text)

If check $=$ False Then

lastval $=$ Val(txtStrainRange. Text) 'value is not valid store last valid number

GoTo errorl 'goto error handling code

Else: Exit Sub 'valid number exit subroutine

End It

ertorl:

MsgBox "You must enter a valid numeric value", vbExclamation 'error message

txtStrainRange. Text $=$ lastval 'set displayed load range to last valid number

\section{End Sub}

Private Sub OKButton_Click()

'check if any values are zero

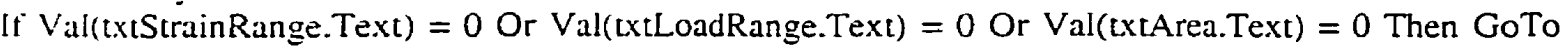
errorl

dlgCalcConst.Hide 'hide dialog box

Exit Sub 'exit subroutine

error 1:

'display message box

MsgBox "You must enter non zero values for Load and Strain Range and X-Sect Area", vbExclamation

Exit Sub 'exit subroutine

End Sub

\section{A3.1.4 dlgYoungsMod}

'all variables must be declared

Option Explicit 


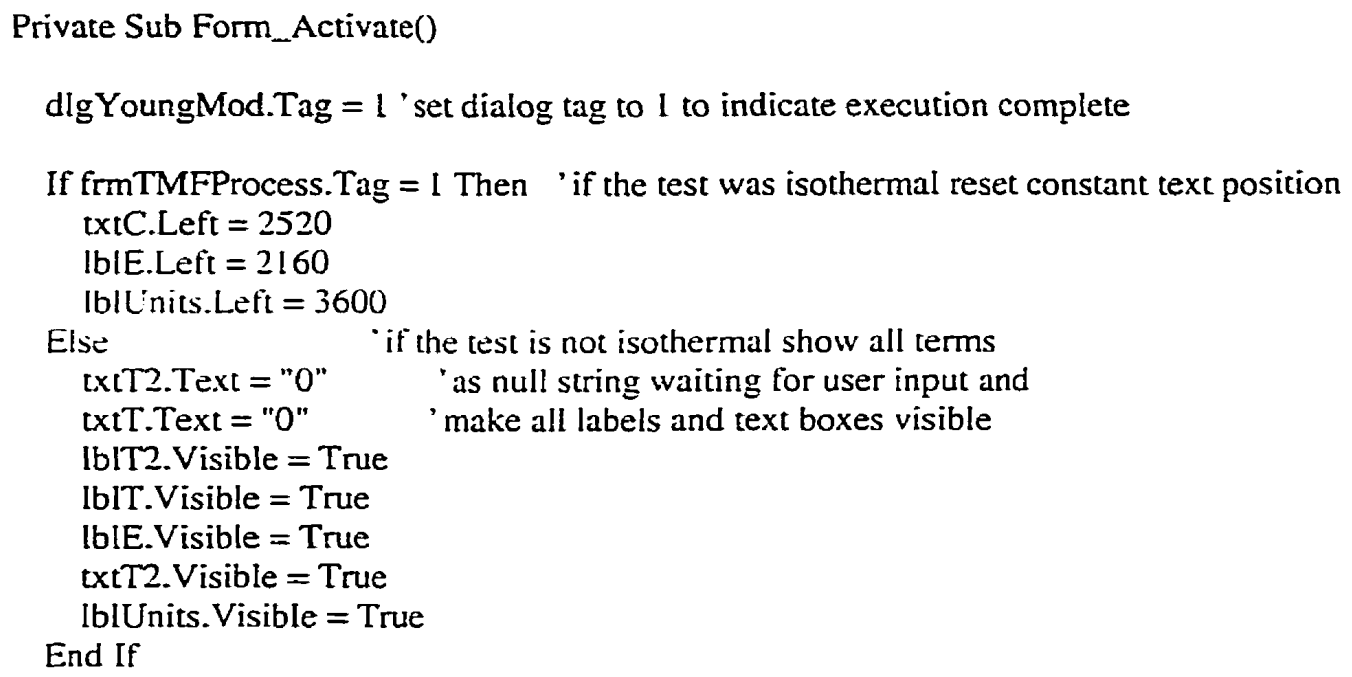

End Sub

Private Sub Form_Load()

dlgYoungMod.Tag $=\mathrm{I}$ 'set dialog tag to $\mathrm{I}$ to indicate execution complete

If frmTMFProcess.Tag $=1$ Then 'if the test was isothermal set $\mathrm{T}^{\wedge} 2$ and $\mathrm{T}$ order txtT. Text $=" 0 " \quad$ terms to 0 and constant term to null string txtT2.Text $=$ "O" 'only label and text box for constant term txtC.Text $=" 0 " \quad$ are visible in this case

IbIT2.Visible $=$ False

lblT . Visible $=$ False txtT2. Visible $=$ False txtT. Visible $=$ False txtC. Left $=2520$ $|\mathrm{b}| \mathrm{E}$. Left $=2160$ lblUnits.Left $=3600$

Else txtT2.Text $=" 0 "$ txtC. Text $=" 0 "$ txtT.Text $=" 0 "$ IbIT2. Visible $=$ True IbIT. Visible $=$ True lblE. Visible $=$ True txtT2. Visible $=$ True txtC. Visible $=$ True lbiUnits. Visible $=$ True

End If

'if the test is not isothermal show all terms

as null string waiting for user input and 'make all labels and text boxes visible

\section{End Sub}

Private Sub OKButton_Click()

If frmTMFProcess. Tag $=1$ Then if test is isothermal after ok button is pressed If $\mathrm{Val}(\mathrm{txtC}$. Text) $=0$ Then 'if constant term is null string call error handling GoTo errorl End If 


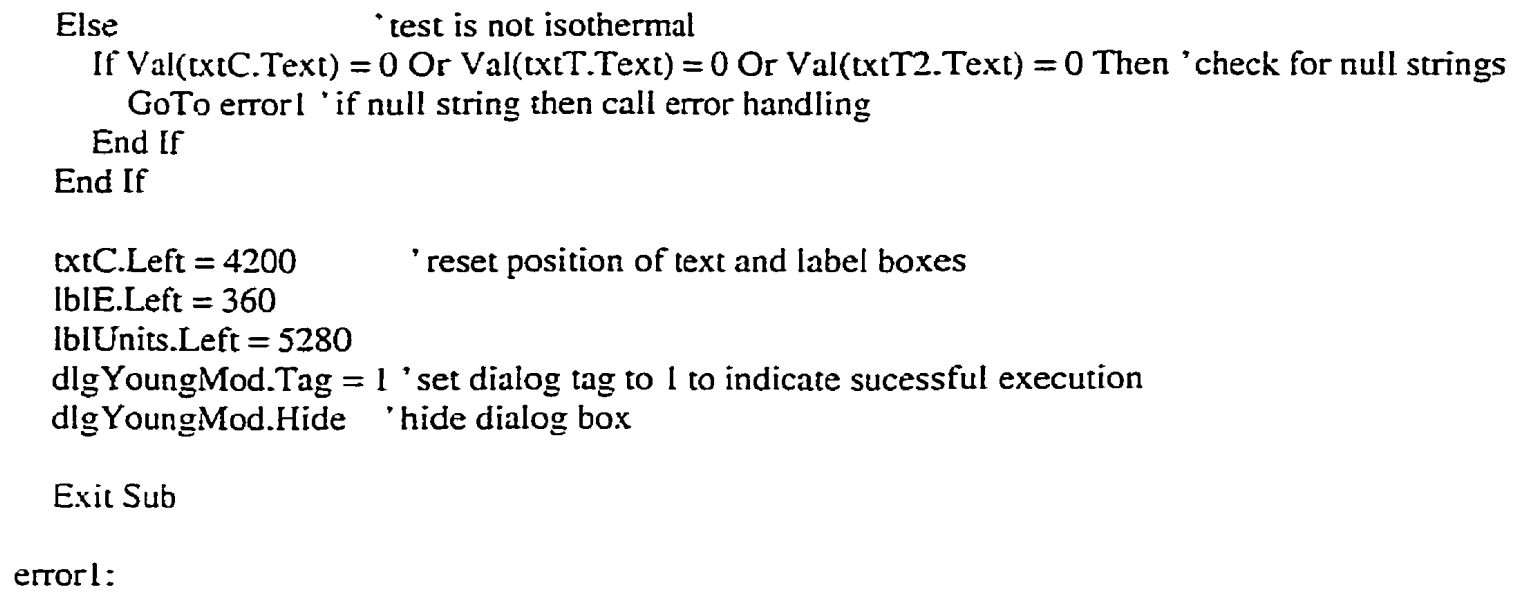

Exil Sub

errorl:

MsgBox "You must enter a non zero Youngs' modulus value", vbExclamation, "Error" "display error message

\section{End Sub}

Private Sub CancelButton_Click()

txtC. Lefi $=+200 \quad$ reset position of text and label boxes

IblE.Left $=360$

lblUnits. Left $=5280$

dlgYoungMod.Tag $=0$ ' set dialog tag value to 0 to indicate cancellation

dlgYoungMod.Hide 'hide dialog box

\section{End Sub}

Private Sub txtC_Change()

Dim check As Boolean

Dim lastval As Single

' check to see if current $T$ value is a valid number check = IsNumeric ( $\mathrm{LxtC}$. Text)

If check $=$ False Then lastval $=$ Val(txtC.Text) $\quad$ value is not valid store last valid number GoTo errorl 'goto error handling code

Else: Exit Sub valid number exit subroutine

End If

crrorl:

MsgBox "You must enter a valid numeric value", vbExclamation error message

extC. Text = lastval 'set displayed text to last valid numeric value

\section{End Sub}

\section{Private Sub txtT_Change()}

Dim check As Boolean

Dim lastval As Single

check to see if current $T$ value is a valid number 
check $=$ IsNumeric(txtT.Text)

If check $=$ False Then

lastval $=$ Val(txtT.Text) ' value is not valid store last valid number

GoTo errorl goto error handling code

Else: Exit Sub "valid number exit subroutine

End If

errorl:

MsgBox "You must enter a valid numeric value", vbExclamation 'error message

txtT.Text = lastval ' set displayed text to last valid numeric value

End Sub

Private Sub txtT2_Change()

Dim check As Boolean

Dim lastval As Single

'check to see if current $T^{\wedge} 2$ value is a valid number

check $=$ IsNumeric $($ txtT2.Text)

If check $=$ False Then

lastval $=$ Val (xiT2.Text) $\quad$ value is not valid store last valid number

GoTo error 1

Else: Exit Sub

'goto error handling code

End If

errorl:

MsgBox "You must enter a valid numeric value", vbExclamation 'error message

txtT2. Text = lastval ' set displayed text to last valid numeric value

End Sub

\section{A3.1.5 frmAbout}

Option Explicit

- Reg Key Sccurity Options...

Const READ_CONTROL $=\& H 20000$

Const KEY_QUERY_VALUE $=\& H I$

Const KEY_SET_VALUE $=\& H 2$

Const KEY_CREATE_SUB_KEY $=\& \mathrm{H}_{4}$

Const KEY_ENUMERATE_SUB_KEYS $=\& \mathrm{H} 8$

Const KEY_NOTIFY $=\&$ HIO

Const KEY_CREATE_LINK $=\& H 20$

Const KEY_ALL_ACCESS $=$ KEY_QUERY_VALUE + KEY_SET_VALUE + KEY_CREATE_SUB_KEY + KEY_ENUMERATE_SUB_KEYS + KEY_NOTIFY + KEY_CREATE_LINK + READ_CONTROL

-Reg Key ROOT Types...

Const HKEY_LOCAL_MACHINE $=\& H 80000002$

Const ERROR_SUCCESS $=0$

Const REG_SZ $=1$

Const REG_DWORD $=4$

- Unicode nul terminated string

-32-bit number 
Const gREGKEYSYSINFOLOC $=$ "SOFTWARELMicrosoft

Const gREGVALSYSINFOLOC = "MSINFO"

Const gREGKEYSYSINFO = "SOFTWAREMMicrosoft IShared ToolsLMSINFO"

Const gREGVALSYSINFO $=$ "PATH"

Private Declare Function RegOpenKeyEx Lib "advapi32" Alias "RegOpenKeyExA" (ByVal hKey As Long. ByVal lpSubKey As String, ByVal ulOptions As Long. ByVal samDesired As Long, ByRef phkResult As Long) As Long

Private Declare Function RegQueryValueEx Lib "advapi32" Alias "RegQueryValueExA" (ByVal hKey As Long, ByVal IpValueName As String, ByVal lpReserved As Long, ByRef IpType As Long, ByVal lpData As String. ByRef lpcbData As Long) As Long

Private Declare Function RegCloseKey Lib "advapi32" (ByVal hKey As Long) As Long

Private Sub cmdSysInfo_Click()

Call StartSysInfo

End Sub

Private Sub cmdOK_Click()

Unload Me

End Sub

Private Sub Form_Load()

Me.Caption = "About" \& App. Title

IblVersion.Caption = "Version " \& App.Major \& "." \& App.Minor \& "." \& App.Revision

lblTitle.Caption $=$ App.Title

End Sub

Public Sub StartSysInfo()

On Error GoTo SysinfoErr

Dim rc As Long

Dim SysInfoPath As String

'Try To Ger System Into Program PathlName From Registry...

If GetKeyValue(HKEY_LOCAL_MACHINE, gREGKEYSYSINFO, gREGVALSYSINFO, SysinfoPath) Then

Try Tu cict Sylem Info Program Path Only From Registry...

Elselt GetKeyValue(HKEY_LOCAL_MACHINE, gREGVALSYSINFOLOC. SysInfoPath) Then

- Validilte Existance Of Known 32 Bit File Version

If (Dir(SysInfoPath \& "IMSINFO32.EXE") $>$ "') Then

SysInfoPath = SysInfoPath \& "LMSINFO32.EXE"

-Error - File Can Not Be Found...

Else

GoTo SysInfoErt

End If

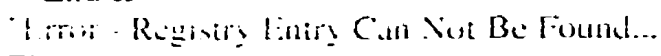

Else

End If

GoTo SysInfoErt

Call Shell(SysInfoPath, vbNormalFocus) 


\section{Exit Sub}

SysInfoErt:

MsgBox "System Information Is Unavailable At This Time", vbOKOnly

End Sub

Public Function GetKeyValue(KeyRoot As Long, KeyName As String, SubKeyRef As String, ByRef KeyVal As String) As Boolean

Dim i As Long

Dim rc As Long

Dim hKey As Long

Dim hDepth As Long

Dim KeyValType As Long

Dim tmpVal As String

Dim KeyValSize As Long

- Loop Counter

- Return Code

'Handle To An Open Registry Key

- Ditat Type Or A Registry Key

- Tempory Storage For A Registry Key Vatue

'Size Of Registry Key Varriable

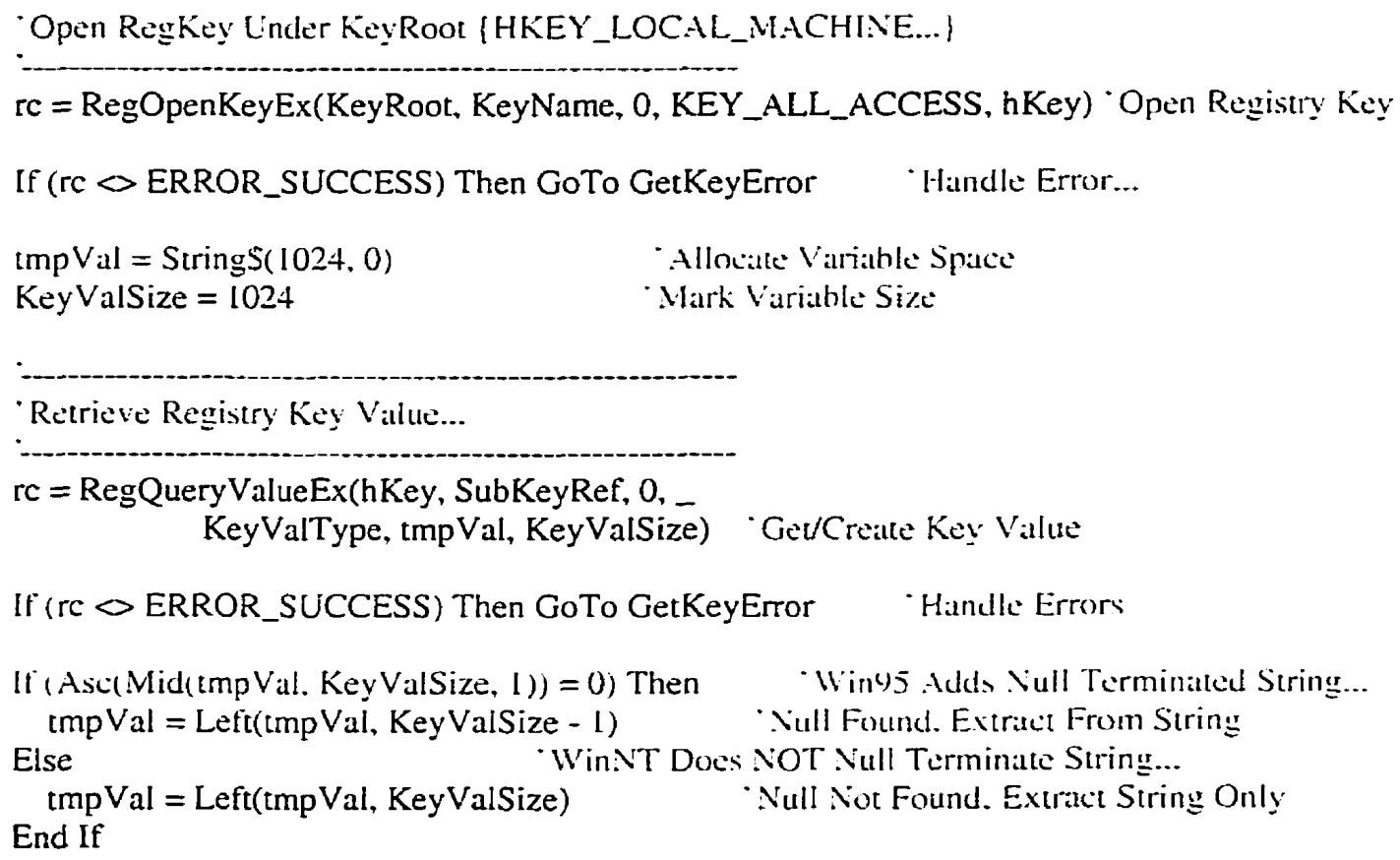

- Determine Key Value Type For Conversion...

$\begin{array}{lc}\text { Select Case KeyValType } & \text { Search Data Types... } \\ \text { Case REG_SZ } & \text { String Registry Key Data Type } \\ \text { KeyVal }=\text { tmpVal } & \text { Copy String Value } \\ \text { Case REG_DWORD } & \text { Douhle Word Registry Key Data Type } \\ \quad \text { For } \mathrm{i}=\text { Len(tmpVal) To I Step }-1 & \text { Convert Each Bit }\end{array}$
Next

KeyVal = KeyVal + Hex(Asc(Mid(tmpVal, i, I)) ${ }^{\prime}$ Build Value Char. By Char.

KeyVal = Format\$("\&h" + KeyVal) "Conven Double Word To String

End Select

GetKeyValue $=$ True $\mathrm{rc}=$ RegCloseKey(hKey)

Exit Function

GetKeyError: 'Cleanup Aticr An Error Hats Occurcd...

KeyVal = "" $\quad$ Sel Retum Val To Empty String
- Return Sucess

- Close Registry Key

Exit 
GetKeyVaiue $=$ False $\mathrm{rc}=$ RegCloseKey(hKey)

End Function
- Return Failure

- Close Registry Key

\section{A3.2 Front Panels}

5. TMF Post Processing Program

$-\square \times$

File Hêlp

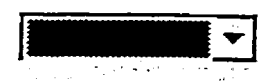

Fiocess

Figure A3. 1 - VB main form for TMF Post Processing Program.

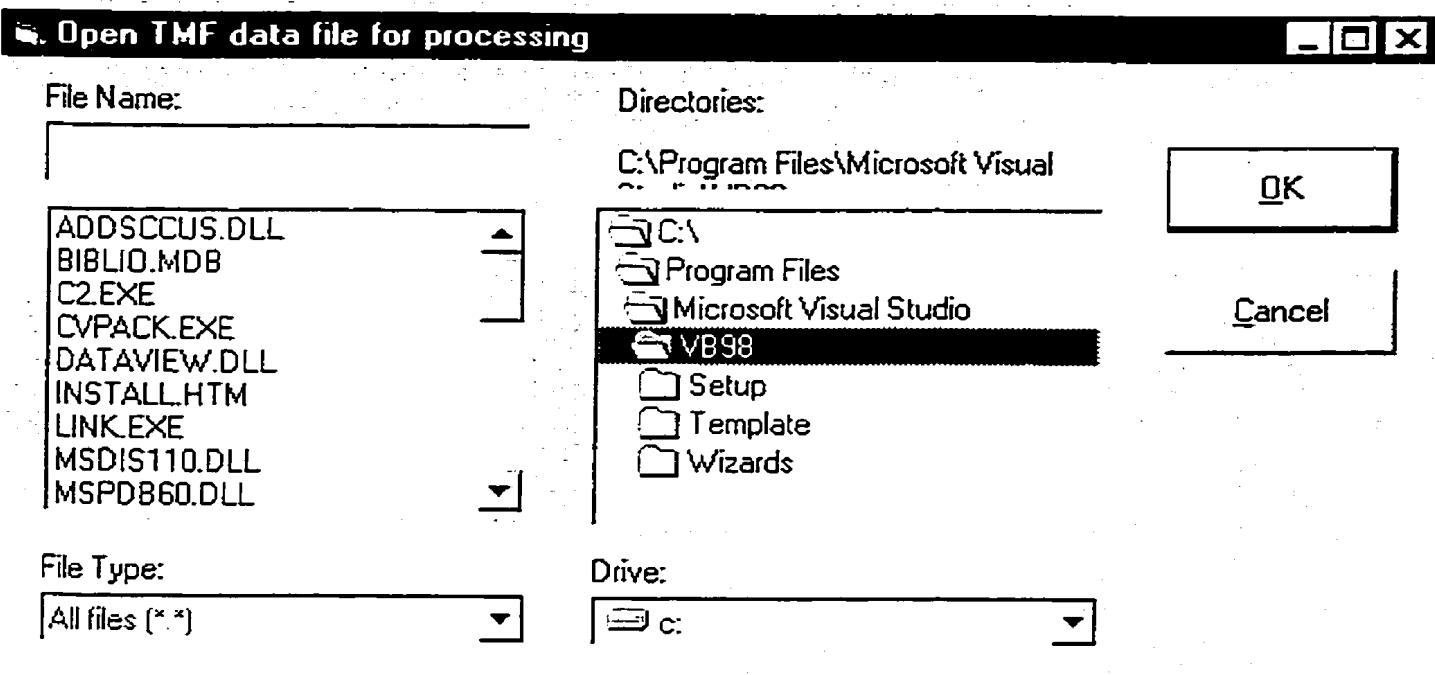

Figure A3. 2 - VB form for selecting the data file to process. 


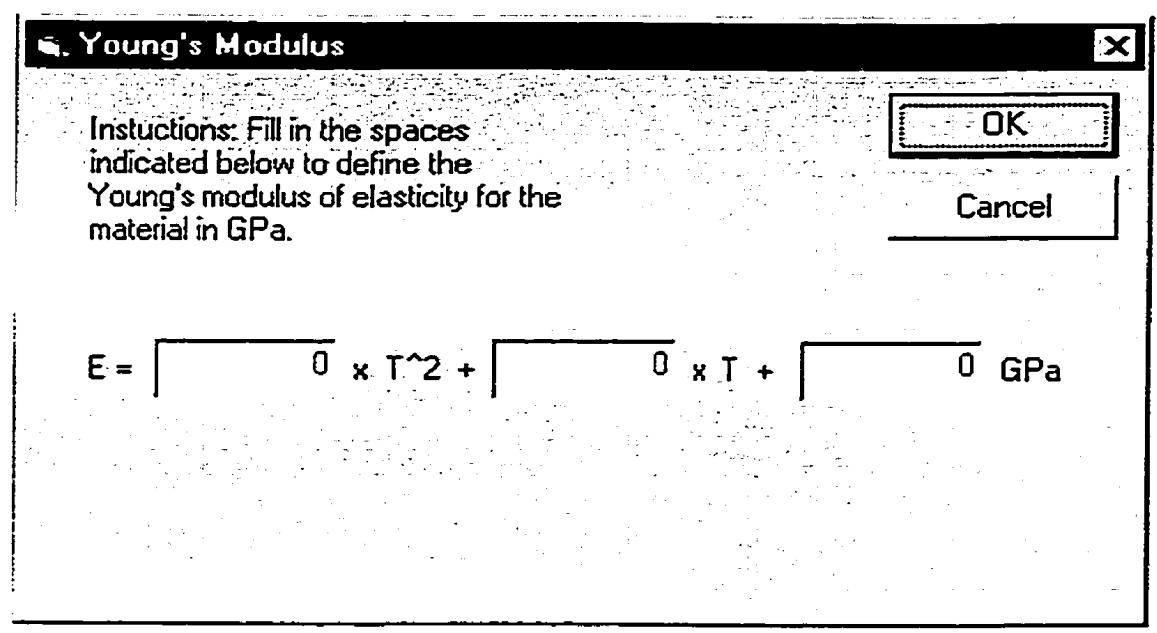

Figure A3. 3 - VB form for inputting the temperature dependent Young's modulus as a function of temperature.

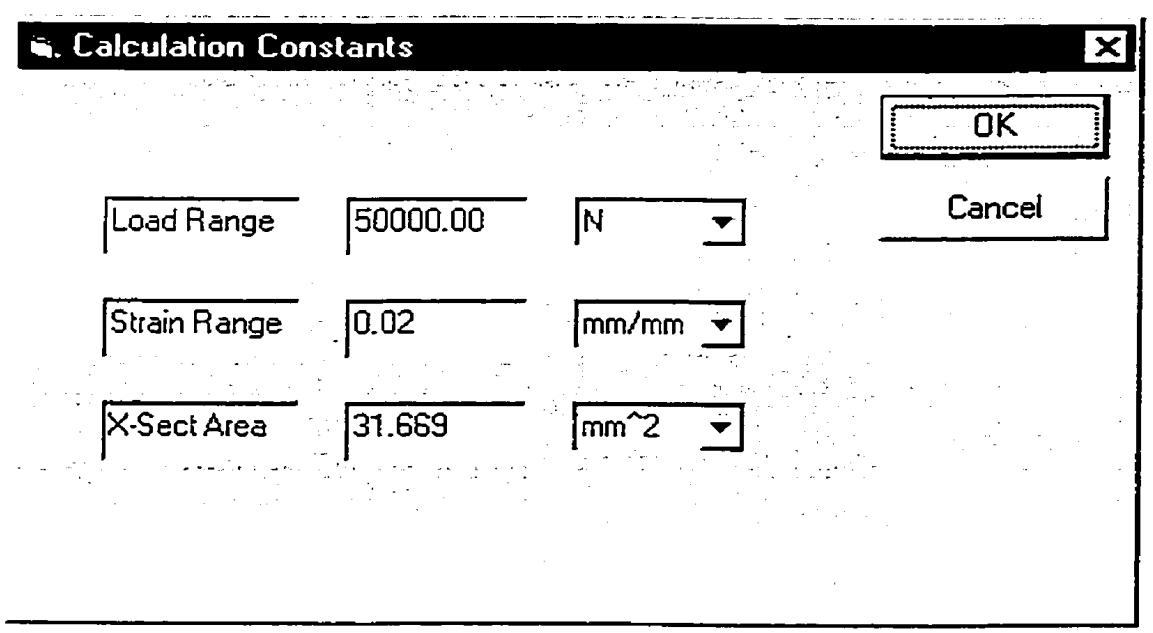

Figure A3. 4 - VB form for inputting the calculation constants for the post processing program. 


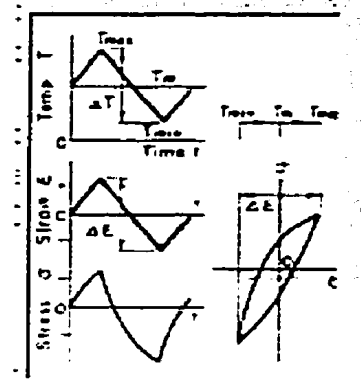

TMF processor

Version 0.1 .4

An application for post processing TMF hysteresis loop daka and peak/valley data recorded during a TMF test into engineering units. The output files are created in a TecPlot ascii .dat file format for import directly into TecPlot.

If any bugs are found contact S. Yandt to correct them.

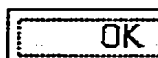

$\vdots$

System Info..

Figure A3. 5 - VB about form. 